\title{
Photochemical Cyclopropenation of Alkynes with Diazirines as Carbene Precursors in Continuous Flow
}

\author{
Nour Tanbouza, Virginie Carreras, and Thierry Ollevier*
}

Département de chimie, Université Laval, 1045 avenue de la Médecine

Québec, QC, G1V 0A6, Canada

\section{Table of Contents}

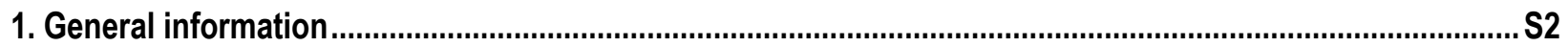

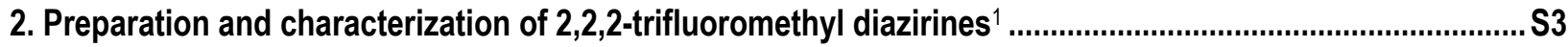

2.1 General procedure for the synthesis of 2,2,2-trifluoromethyl O-tosyl oximes .............................................S3

2.2 General procedure for the synthesis of 2,2,2-trifluoromethyl diaziridines.....................................S8

2.3. Procedure for synthesis of 2,2,2-trifluoromethyl diazirines 1a-n ...........................................S11

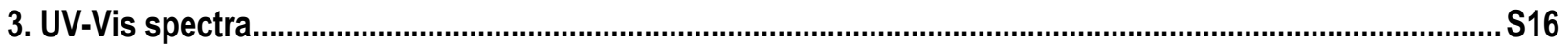

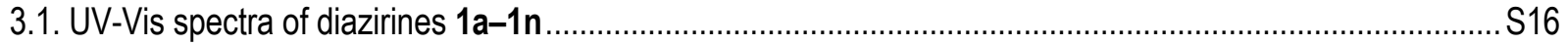

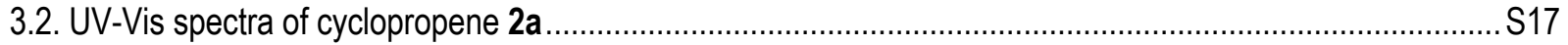

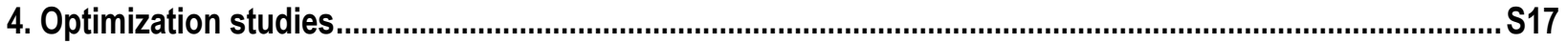

4.1 Screening of photochemical cyclopropenation with medium-pressure mercury lamp: ................................S17

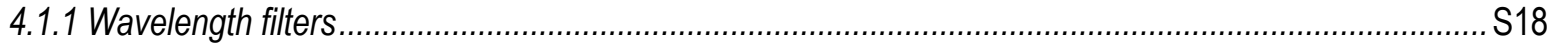

4.2 Screening of flow conditions for the cyclopropenation of diazirine 1a...................................................... S18

4.2.1 Screening of photochemical cyclopropenation with light-emitting diodes (LEDs) ...................................S18

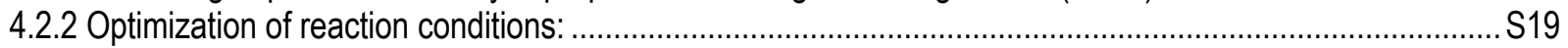

5. Experimental procedures for the cyclopropenation reaction of diazirines in continuous flow..................S20

5.1 Continuous flow setup for cyclopropene synthesis ............................................................................... S20

5.2 General procedure for the photochemical flow reactions of diazirines in continuous flow .............................S21

5.3 General procedure for the photochemical reactions of diazirines in batch ............................................... S28

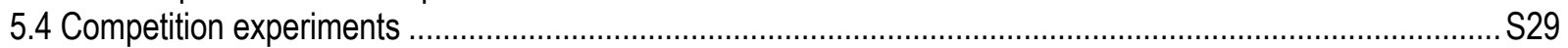

5.4.1 General procedure for the competition reaction between internal and terminal alkynes ..........................S29

5.4.2 General procedure for the competition reaction between internal alkynes and alkenes............................S30

5.4 .3 General procedure for the competition reaction between internal alkynes and alkenes............................S31

5.5 General procedure for the TMS deprotection of 2ak .............................................................................. 32

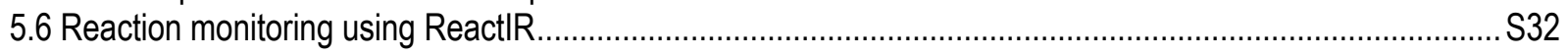

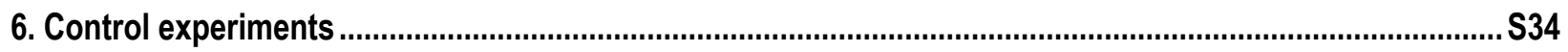

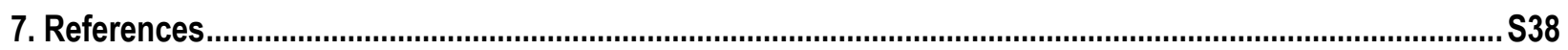

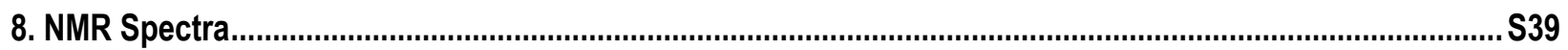




\section{General information}

All reactions were carried out in flame-dried glassware under an atmosphere of argon with magnetic stirring. All flow reactions were performed using a Vapourtec E-series easy-MedChem flow system using V-3 peristaltic pumps and PFA (polyfluoroalkoxy polymer) tubing $(1.3 \times 0.15 \mathrm{~mm}$ bore and wall) equipped with a UV-150 photochemical reactor kit using standard high efficiency LED (light-emitting diodes) and medium-pressure mercury reactor kit. Light sources used with the flow reactor are the commercial Gen-1 Vaportec units (see full details at: https://www.vapourtec.com/products/flow-reactors/photocatalysis-led/). The photochemical unit is temperaturecontrolled with a Vapourtec Cooled Module accessory. Batch photochemical reactions were done in borosilicate glass vials using LED strips (Govee 16.4 Feet RGB LED Light Strip, H61271A2 ,12 V, 24 watts) wrapped around a cardboard box (distance from vial: $5 \mathrm{~cm}$ edges $\times 2 \mathrm{~cm}$ top $\times 0 \mathrm{~cm}$ bottom) and cooled with a flow of compressed air. All starting material purchased from commercial suppliers were used without further purification. Oil baths (mineral oil) were used for reactions that required heating. Thin-layer chromatography (TLC) was carried out on $250 \mu \mathrm{m}$ commercial silica gel plates and compounds were visualized using UV absorbance $(254 \mathrm{~nm})$ and/or aqueous $\mathrm{KMnO}_{4}$. Solvents were dried according to standard procedures. Automated flash column chromatography was performed on Biotage ${ }^{\circledR} \mid$ solera One $^{\top M}$ (SNAP Ultra cartridge). ${ }^{1} \mathrm{H},{ }^{13} \mathrm{C}\left\{{ }^{1} \mathrm{H}\right\}$, and ${ }^{19} \mathrm{~F}$ NMR spectra were recorded on Varian Inova $400 \mathrm{MHz}$ and Agilent DD2 $500 \mathrm{MHz}$ spectrometers in $\mathrm{CDCl}_{3}$. For ${ }^{1} \mathrm{H}$ NMR, chemical shifts are reported as follows: chemical shift (in ppm), multiplicity ( $\mathrm{s}=$ singlet, $\mathrm{d}=$ doublet, $\mathrm{t}=$ triplet, $\mathrm{q}=$ quartet, $\mathrm{m}=$ multiplet), coupling constant (in $\mathrm{Hz}$ ), and integration. For ${ }^{13} \mathrm{C}\left\{{ }^{1} \mathrm{H}\right\} \mathrm{NMR}, \mathrm{CDCl}_{3}$ was used as internal standard $(\delta=77.16 \mathrm{ppm})$ and spectra were obtained with complete proton decoupling. For ${ }^{19} F$ NMR, no external standard was used. High-resolution mass spectra (HRMS) were recorded on LC/ESI-TOF (time-of-flight) mass spectrometer Agilent 6210 mass spectrometer using electrospray ionization (ESI). IR spectra were recorded on a NICOLET 380 FT-IR spectrometer with a ZnSe ATR accessory and are reported in reciprocal centimeter $\left(\mathrm{cm}^{-1}\right)$. Reaction monitoring with IR was performed using Mettler Toledo FlowIR ${ }^{\mathrm{TM}}$ cell with a silicon probe and recorded on a ReactIR ${ }^{\mathrm{TM}} 15$ apparatus and data is processed on iC IR software. Melting points (mp) are uncorrected and were recorded on a MEL-TEMP ${ }^{\circledR}$ melting point apparatus. UV-Vis were recorded on an Agilent Cary 7000 Series UV-Vis-NIR spectrophotometer using $\mathrm{CH}_{2} \mathrm{Cl}_{2}$ as a blank and processed on Cary WinUV software. 


\section{Preparation and characterization of 2,2,2-trifluoromethyl diazirines ${ }^{1}$}

Diazirine substrates (1a-1n) are prepared following reported procedures.

\subsection{General procedure for the synthesis of 2,2,2-trifluoromethyl 0-tosyl oximes}

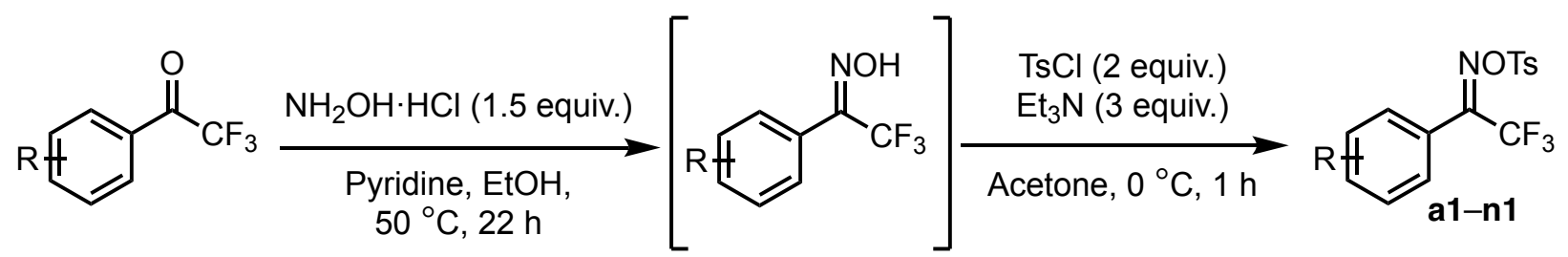

Hydroxylamine hydrochloride $(1.2 \mathrm{~g}, 15 \mathrm{mmol})$ was added to a solution of the 2,2,2-trifuoromethyl ketone $(15 \mathrm{mmol})$ in pyridine $(7.5 \mathrm{~mL})$ and $\mathrm{EtOH}(4 \mathrm{~mL})$. The reaction mixture was heated to $50^{\circ} \mathrm{C}$ overnight. The reaction was cooled to room temperature and diluted with EtOAc, washed with $2 \mathrm{M}$ aq. $\mathrm{HCl}$, dried over $\mathrm{MgSO}_{4}$, and concentrated under reduced pressure. The crude product was used as is for the next step.

The crude 2,2,2-trifuoromethyl oxime was dissolved in acetone $(80 \mathrm{~mL})$ and cooled to $0{ }^{\circ} \mathrm{C}$, to which $\mathrm{Et}_{3} \mathrm{~N}(6 \mathrm{~mL})$ was added followed by tosyl chloride $(3.2 \mathrm{~g}, 16.8 \mathrm{mmol})$. The reaction mixture was brought to room temperature and stirred for $1 \mathrm{~h}$. The reaction was concentrated under reduced pressure. The crude residue was purified by flash chromatography (Biotage, SNAP ULTRA, 9:1 hexanes/EtOAc) to give the O-tosyl oxime of a mixture of both E- and Zisomers.

\subsubsection{Characterization of 0-tosyl oximes (a1-n1)}

1-([1,1'-Biphenyl]-4-yl)-2,2,2-trifluoroethan-1-one 0-tosyl oxime a1

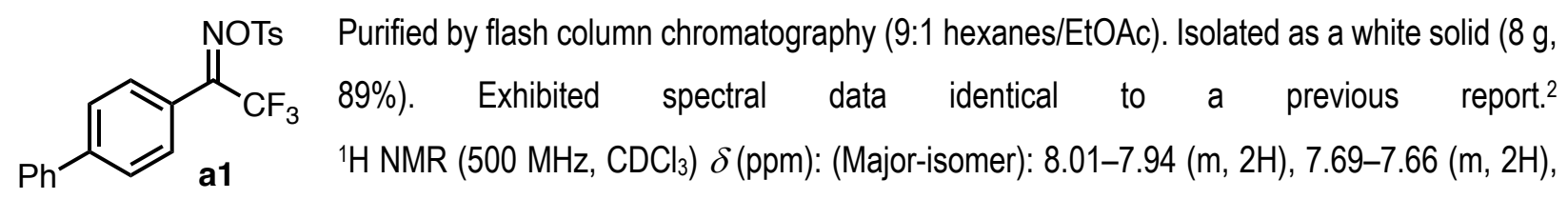
7.59-7.54 (m, 4H), 7.47-7.39 (m, 5H), 2.48 (s, 3H); (Minor-isomer): 7.98 (m, 2H), 7.74-7.68 (m, 2H), 7.66-7.60 (m, 4H), $7.50(\mathrm{~m}, 5 \mathrm{H}), 2.50(\mathrm{~s}, 3 \mathrm{H}) .{ }^{13} \mathrm{C}\left\{{ }^{1} \mathrm{H}\right\} \mathrm{NMR}\left(101 \mathrm{MHz}, \mathrm{CDCl}_{3}\right) \delta$ (ppm): (Major-isomer): 153.8 (q, J=31.5 Hz), 146.3, 144.8, 139.5, 131.4, 130.0 (q, J = 2.4 Hz), 129.3, 129.1, 128.0, 127.4, 127.2, 123.2, 117.5 (q, J = 285.6 Hz), 21.8; (Minor-isomer): 153.8 (q, $J=31.5 \mathrm{~Hz}$ ), 146.4, 144.6, 139.5, 131.2, 129.4, 129.2, 128.9, 128.4 (q, J = 2.0 Hz), 127.7, 127.5, 127.3, 126.5, 119.8 (q, J = 277.3 Hz), 21.8. $\left.{ }^{19} \mathrm{~F} \mathrm{NMR} \mathrm{(470} \mathrm{MHz,} \mathrm{CDCl}_{3}\right) \delta$ (ppm): -61.4 (Minor-isomer), -66.5 (Major-isomer). 


\section{2,2,2-Trifluoro-1-(naphthalen-2-yl)ethan-1-one 0-tosyl oxime b1}

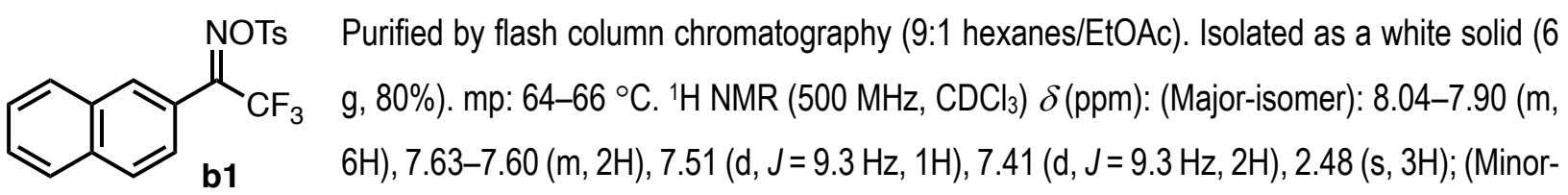

isomer): $7.90-7.81(\mathrm{~m}, 6 \mathrm{H}), 7.59-7.54(\mathrm{~m}, 2 \mathrm{H}), 7.46(\mathrm{~d}, \mathrm{~J}=8.6 \mathrm{~Hz}, 1 \mathrm{H}), 7.38(\mathrm{~d}, \mathrm{~J}=8.4 \mathrm{~Hz}, 2 \mathrm{H}), 2.46(\mathrm{~s}, 3 \mathrm{H}) .{ }^{13} \mathrm{C}\left\{{ }^{1} \mathrm{H}\right\}$ NMR (126 MHz, $\left.\mathrm{CDCl}_{3}\right) \delta$ (ppm): (Major-isomer): 154.5 (q, $\left.J=33.8 \mathrm{~Hz}\right), 146.3,134.4,133.9,132.7,132.4,131.4$, 130.0 (q, $J=1.6 \mathrm{~Hz}$ ), 130.0, 129.2, 125.0, 124.5, 119.8 (q, $J=278.0, \mathrm{~Hz}$ ), 21.8; (Minor-isomer): 154.1 (q, $J=32.2 \mathrm{~Hz}$ ), 146.2, 134.3, 133.8, 132.6, 132.4, 131.2, 129.3, 129.2 (q, $J=1.6 \mathrm{~Hz}), 128.9,124.9,124.4,116.4$ (q, $J=283.3 \mathrm{~Hz}$ ), 21.8. ${ }^{19} \mathrm{~F} \mathrm{NMR}\left(470 \mathrm{MHz}, \mathrm{CDCl}_{3}\right) \delta(\mathrm{ppm})$ : -61.2 (Minor-isomer), -66.4 (Major-isomer). IR ( $\left.\mathrm{cm}^{-1}\right): 3256,3064,1596$, 1377, 1194, 1148, 1095, 872, 750. HR-MS (EI) $\mathrm{m} / \mathrm{z}[\mathrm{M}+\mathrm{H}]^{+}$calcd. for $\mathrm{C}_{19} \mathrm{H}_{15} \mathrm{~F}_{3} \mathrm{NO}_{3} \mathrm{~S} 394.0719$, found 394.0749.

\section{1-(4-(Benzyloxy)phenyl)-2,2,2-trifluoroethan-1-one 0-tosyl oxime c1}<smiles>[O+]N=C(c1ccc(OCc2ccccc2)cc1)C(F)(F)F</smiles>

Purified by flash column chromatography ( $9: 1$ hexanes/EtOAc). Isolated as a white solid (932 mg, 42\%). mp: $78-80{ }^{\circ} \mathrm{C} .{ }^{1} \mathrm{H}$ NMR (500 MHz, $\left.\mathrm{CDCl}_{3}\right) \delta$ (ppm): (Major-isomer): 7.957.87 (m, 2H), 7.42-7.33 (m, 9H), 7.03-6.99 (m, 2H), 5.12 (s, 2H), 2.48 (s, 3H); (Minorisomer): 7.50-7.41 (m, 11H), 7.08-7.04 (m, 2H), $5.13(\mathrm{~s}, 2 \mathrm{H}), 2.49(\mathrm{~s}, 3 \mathrm{H}) .{ }^{13} \mathrm{C}\left\{{ }^{1} \mathrm{H}\right\} \mathrm{NMR}$ (126 MHz, $\mathrm{CDCl}_{3}$ ) $\delta$ (ppm): (Major-isomer): 161.7, 153.6 (q, $\left.J=31.9 \mathrm{~Hz}\right), 146.0,136.1,131.6,130.7$ (q, J = $1.5 \mathrm{~Hz}$ ), 129.9, 129.1, 128.8, 128.3, 127.5, 117.5 (q, $J=284.4 \mathrm{~Hz}$ ), 116.7, 115.1, 70.2, 21.8; (Minor-isomer): 161.3, 153.2 (q, $J$ $=32.8 \mathrm{~Hz}$ ), 146.1, 136.1, 131.2, $130.8(\mathrm{~d}, \mathrm{~J}=1.1 \mathrm{~Hz}), 129.9,129.3,128.8,128.4,127.5,120.1,119.9$ (q, J = 278.0

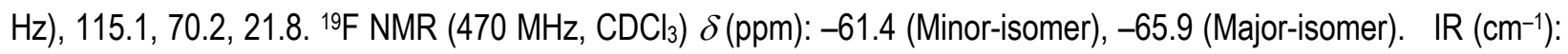
$3070,2926,1599,1496,1390,1177,1140,812,735$. HR-MS (EI) m/z [M + H] calcd. for $\mathrm{C}_{22} \mathrm{H}_{19} \mathrm{~F}_{3} \mathrm{NO}_{4} \mathrm{~S} 450.0981$ found 450.0966 .

\section{1,1'-([1,1'-Biphenyl]-4,4'-diyl)bis(2,2,2-trifluoroethan-1-one) 0,0-ditosyl dioxime d1}

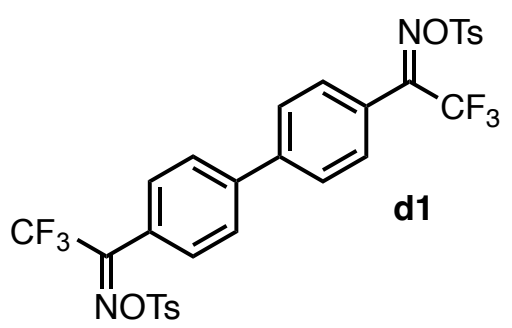

Purified by flash column chromatography ( $9: 1$ hexanes/EtOAc). Isolated as a white solid ( $1.6 \mathrm{~g}, 94 \%)$ by doubling the quantities of the above procedure. m.p. 50-52 ${ }^{\circ} \mathrm{C} . \mathrm{mp}: 64-66^{\circ} \mathrm{C} .{ }^{1} \mathrm{H} \mathrm{NMR}\left(500 \mathrm{MHz}, \mathrm{CDCl}_{3}\right) \delta$ (ppm): (Major-isomer): 7.97-7.90 (m, 3H), 7.75-7.63 (m, 5H), 7.55 (m, 4H), 7.45-7.39 (m, 4H), 2.48 (s, 6H); (Minor-isomer): 7.97-7.90 (m, 3H), 7.69 (d, J = 8.1 Hz, 5H), 7.58-7.51 (m, 4H), 7.44-7.34 (m, 4H), 2.49 (s, 3H). ${ }^{13} \mathrm{C}\left\{{ }^{1} \mathrm{H}\right\}$ NMR (126 MHz, $\left.\mathrm{CDCl}_{3}\right) \delta(\mathrm{ppm}):$ (Major-isomer): 153.5 (q, J = 31.8 $\mathrm{Hz}$ ), 146.3, 142.9, 132.2, 131.3, 130.0, 129.6 (d, $J=1.3 \mathrm{~Hz}$ ), 127.5, 124.3, 118.5 (q, $J=285.2 \mathrm{~Hz}$ ), 21.8; (Majorisomer): 153.5 (q, $J=33.5 \mathrm{~Hz}$ ), 146.4, 142.9, 132.0, 131.0, 129.2 (d, $J=1.1 \mathrm{~Hz}), 129.2,127.5,127.0,119.6$ (q, $J=$ 278.1 Hz), 21.8. ${ }^{19} \mathrm{~F} \mathrm{NMR}\left(470 \mathrm{MHz}, \mathrm{CDCl}_{3}\right) \delta(\mathrm{ppm}):-61.4$ (Minor-isomer), -66.5 (Major-isomer). IR (cm-1): 3097 , $2874,1597,1391,1192,1153,1092,885,814$. HR-MS (EI) $\mathrm{m} / \mathrm{z}\left[\mathrm{M}+\mathrm{NH}_{4}\right]^{+}$calcd. for $\mathrm{C}_{30} \mathrm{H}_{26} \mathrm{~F}_{6} \mathrm{~N}_{3} \mathrm{O}_{6} \mathrm{~S}_{2} 702.1162$ found 702.1144 . 


\section{2,2,2-Trifluoro-1-(p-tolyl)ethan-1-one 0-tosyl oxime e1}

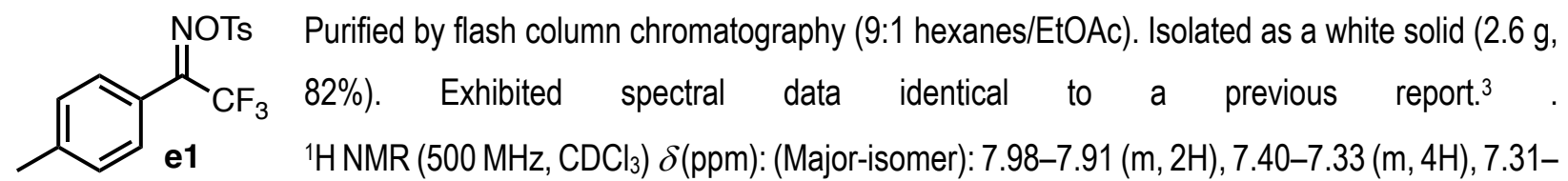
7.25 (m, 2H), 2.39 (s, 1H), 2.38 (s, 1H); (Minor-isomer): 7.98-7.91 (m, 1H), 7.51-7.44 (m, 1H), 7.32-7.25 (m, 4H), 2.45 $(\mathrm{s}, 1 \mathrm{H}), 2.44$ (s, 1H). ${ }^{13} \mathrm{C}\left\{{ }^{1} \mathrm{H}\right\} \mathrm{NMR}\left(126 \mathrm{MHz}, \mathrm{CDCl}_{3}\right) \delta$ (ppm): (Major-isomer): 154.2 (q, J = $\left.32.3 \mathrm{~Hz}\right), 146.4,142.5$, 131.2, 130.0, 129.5, 129.2, 128.8 (q, $J=1.4 \mathrm{~Hz}$ ), 121.6, 119.8 (q, $J=277.5 \mathrm{~Hz}$ ), 21.4, 21.3; (Minor-isomer): 147.3 (q, $J=32.5 \mathrm{~Hz}$ ), 146.3, 142.7, 131.4, 129.5, 129.2, 129.1, 128.6 (q, $J=1.3 \mathrm{~Hz}), 124.8,117.5$ (q, $J=284.9 \mathrm{~Hz}), 21.6,21.6$. ${ }^{19} \mathrm{~F} \mathrm{NMR}\left(470 \mathrm{MHz}, \mathrm{CDCl}_{3}\right) \delta$ (ppm): -61.5 (Minor-isomer), -66.5 (Major-isomer).

\section{1-(4-(Tert-butyl)phenyl)-2,2,2-trifluoroethan-1-one 0-tosyl oxime f1}

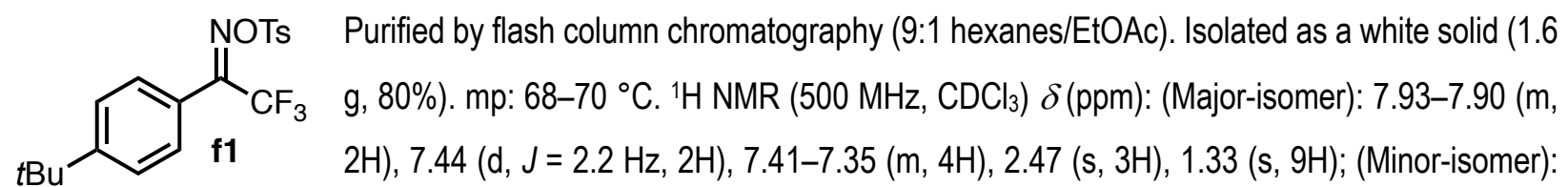
7.94-7.89 (m, 2H), 7.51-7.48 (m, 2H), 7.42-7.37 (m, 4H), 2.49 (s, 3H), 1.35 (s, 6H). ${ }^{13} \mathrm{C}\left\{{ }^{1} \mathrm{H}\right\} \mathrm{NMR}\left(126 \mathrm{MHz}, \mathrm{CDCl}_{3}\right)$ $\delta$ (ppm): (Major-isomer): 155.5, 154.0 (q, $J=32.1 \mathrm{~Hz}$ ), 145.9, 131.5, 129.9, 129.1, 128.7 (q, $J=1.2 \mathrm{~Hz}), 125.8,121.6$, 117.4 (q, $J=283.9 \mathrm{~Hz}$ ), 35.0, 31.0, 21.8; (Minor-isomer): 155.3, 153.9 (q, $J=33.0 \mathrm{~Hz}$ ), 146.1, 131.2, 129.9, 129.3, 128.4 (q, $J=1.2 \mathrm{~Hz}), 125.8,124.8,119.7$ (q, $J=277.7 \mathrm{~Hz}), 35.0,31.0,21.8 .{ }^{19} \mathrm{~F} \mathrm{NMR}\left(470 \mathrm{MHz}, \mathrm{CDCl}_{3}\right) \delta(\mathrm{ppm}):-$ 61.5 (Minor-isomer), -66.4 (Major-isomer). IR ( $\left.\mathrm{cm}^{-1}\right): 2968,2872,1595,1396,1196,1389,1150,729$. HR-MS (EI) m/z $\left[\mathrm{M}+\mathrm{NH}_{4}\right]^{+}$calcd. for $\mathrm{C}_{19} \mathrm{H}_{24} \mathrm{~F}_{3} \mathrm{~N}_{2} \mathrm{O}_{3} \mathrm{~S} 417.1454$ found 417.1413 .

\section{2,2,2-Trifluoro-1-(4-methoxyphenyl)ethan-1-one 0-tosyl oxime g1}

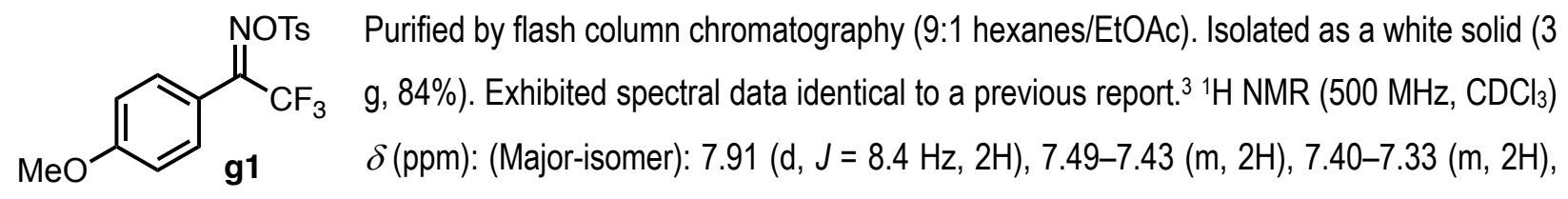
6.95-6.89 (m, 2H), 3.84 (s, 3H), 2.46 (s, 3H); (Minor-isomer): 7.90 (d, J=8.4 Hz, 2H), 7.41 (dt, J1= $7.9 \mathrm{~Hz}, J^{2}=1.1 \mathrm{~Hz}$, 2H), 7.00-6.94 (m, 2H), 3.85 (s, 3H), $2.48(\mathrm{~s}, 3 \mathrm{H}) .{ }^{13} \mathrm{C}\left\{{ }^{1} \mathrm{H}\right\}$ NMR (126 MHz, $\left.\mathrm{CDCl}_{3}\right) \delta(\mathrm{ppm}):($ Major-isomer): 162.5, 153.6 (q, $J=31.8 \mathrm{~Hz}), 145.9,131.5,130.7$ (q, $J=1.6 \mathrm{~Hz}), 129.9,129.1,118.7$ (q, J = 284.5 Hz), 116.4, 114.2, 55.5, 21.8; (Minor-isomer): 162.1, 153.2 (q, $J=32.8 \mathrm{~Hz}), 146.1,131.2,130.7$ (q, $J=1.3 \mathrm{~Hz}), 129.9,129.3,119.8$ (q, $J=$ 277.9 Hz), 119.8, 114.2, 55.4, 21.8. $\left.{ }^{19} \mathrm{~F} \mathrm{NMR} \mathrm{(470} \mathrm{MHz,} \mathrm{CDCl}_{3}\right) \delta(\mathrm{ppm})$ : -61.4 (Minor-isomer), -66.0 (Major-isomer). 


\section{2,2,2-Trifluoro-1-phenylethan-1-one 0-tosyl oxime h1}

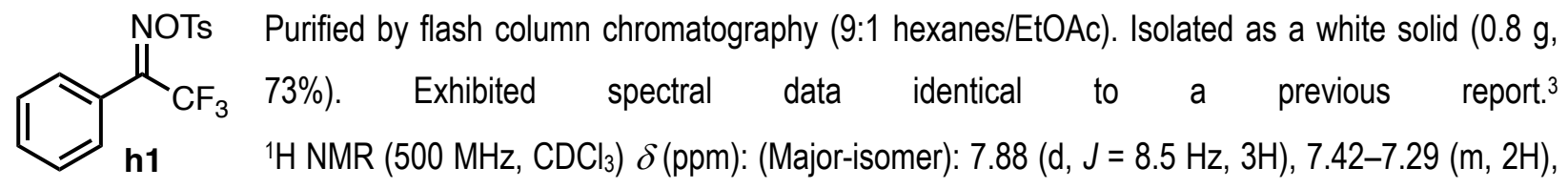
2.45 (s, 3H); (Minor-isomer): 7.90 (d, J=8.8 Hz, 3H), 7.48-7.41 (m, 2H), $2.43(\mathrm{~s}, 3 \mathrm{H}) .{ }^{13} \mathrm{C}\left\{{ }^{1} \mathrm{H}\right\} \mathrm{NMR}\left(126 \mathrm{MHz}, \mathrm{CDCl}_{3}\right)$ $\delta$ (ppm): (Major-isomer): 154.1 (q, $J=33.4 \mathrm{~Hz}$ ), 147.1, 146.5, 131.8, 131.1, 130.4, 129.2, 128.9, 128.3 (q, J = $1.2 \mathrm{~Hz}$ ), 119.7 (q, $J=277.6 \mathrm{~Hz}$ ), 21.7; (Minor-isomer): 154.0 (q, J = 32.2 Hz), 146.4, 141.5, 131.9, 131.3, 129.1, 128.8, 128.8 (q, $J=1.4 \mathrm{~Hz}$ ), 127.6, 117.4 (q, $J=285.2 \mathrm{~Hz}$ ), 21.7. ${ }^{19} \mathrm{~F} \mathrm{NMR}\left(470 \mathrm{MHz}, \mathrm{CDCl}_{3}\right) \delta$ (ppm): -61.4 (Minor-isomer), -66.7 (Major-isomer).

\section{2,2,2-Trifluoro-1-(4-fluorophenyl)ethan-1-one 0-tosyl oxime i1}

NOTs Purified by flash column chromatography (9:1 hexanes/EtOAc). Isolated as a semi solid $(3.9 \mathrm{~g}$,
$\left.\begin{array}{ll}\mathrm{CF}_{3} & 77 \%) .{ }^{1} \mathrm{H} \text { NMR }(500 \mathrm{MHz}, \mathrm{CDCl} \\ 3\end{array}\right) \delta(\mathrm{ppm})$ : (Major-isomer): 7.90-7.89 (m, 2H), 7.46-7.44 (m, $7.88(\mathrm{~m}, 2 \mathrm{H}), 7.43-7.42(\mathrm{~m}, 2 \mathrm{H}), 7.35$ (s br, 2H), 7.10-7.05 (m, 2H), $2.40(\mathrm{~s}, 3 \mathrm{H}) .{ }^{13} \mathrm{C}\left\{{ }^{1} \mathrm{H}\right\} \mathrm{NMR}\left(126 \mathrm{MHz}, \mathrm{CDCl}_{3}\right)$ $\delta$ (ppm): (Major-isomer): 164.8 (d, $J=254.1 \mathrm{~Hz}), 153.0$ (q, $J=32.2 \mathrm{~Hz}), 146.5,131.2$ (d, $J=7.9 \mathrm{~Hz}$ ), 130.0, 129.0, 123.8, 119.5 (q, $J=256.2 \mathrm{~Hz}$ ), 116.2, 116.0, 21.5; (Minor-isomer): 164.1 (d, $J=253.3 \mathrm{~Hz}$ ), 153.0 (q, $J=33.8 \mathrm{~Hz}$ ), 146.4, 131.0 (d, $J=8.4 \mathrm{~Hz}$ ), 130.0, 129.1, 123.7, 119.5 (q, $J=260.2 \mathrm{~Hz}), 116.3,116.1,21.5 .{ }^{19} \mathrm{~F}$ NMR (470 MHz, $\left.\mathrm{CDCl}_{3}\right) \delta$ (ppm): -61.7 (Major-isomer), -66.8 (Minor-isomer) -106.2 (m, Minor-isomer), -106.7 (m, Major-isomer). IR $\left(\mathrm{cm}^{-1}\right): 3074,2874,1599,1512,1389,1150,729$. HR-MS (El) m/z [M + NH$\left.]_{4}\right]^{+}$calcd. for $\mathrm{C}_{15} \mathrm{H}_{15} \mathrm{~F}_{3} \mathrm{~N}_{2} \mathrm{O}_{3} \mathrm{~S} 379.0434$ found 379.0715 .

\section{1-(4-Bromophenyl)-2,2,2-trifluoroethan-1-one 0-tosyl oxime j1}

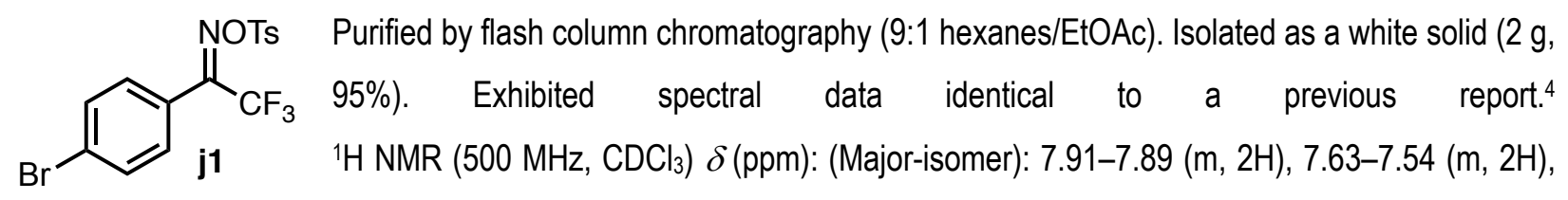
7.42-7.37 (m, 2H), 7.33-7.28 (m, 2H), 2.46 (s, 3H); (Minor-isomer): 7.89-7.87 (m, 2H), 7.57-7.49 (m, 2H), 7.40-7.35 $(\mathrm{m}, 2 \mathrm{H}), 7.28(\mathrm{~d}, J=8.5 \mathrm{~Hz}, 2 \mathrm{H}), 2.44(\mathrm{~s}, 3 \mathrm{H}) .{ }^{13} \mathrm{C}\left\{{ }^{1} \mathrm{H}\right\} \mathrm{NMR}\left(126 \mathrm{MHz}, \mathrm{CDCl}_{3}\right) \delta(\mathrm{ppm})$ : (Major-isomer): 153.0 (q, $J=$ $33.5 \mathrm{~Hz}$ ), 146.4, 141.5, 132.2, 131.2, 130.1 (q, $J=1.1 \mathrm{~Hz}$ ), 129.2, 126.6, 123.3, 117.2 (q, $J=284.1 \mathrm{~Hz}$ ), 21.7; (Minorisomer): 153.0 (q, $J=34.4 \mathrm{~Hz}$ ), 146.5, 146.4, 132.1, 130.9, 130.3 (q, $J=1.1 \mathrm{~Hz}), 129.2,127.0,126.9,119.5$ (q, $J=$ 277.6 Hz), 21.7. ${ }^{19} \mathrm{~F} \mathrm{NMR}\left(470 \mathrm{MHz}, \mathrm{CDCl}_{3}\right) \delta(\mathrm{ppm}):-61.5$ (Minor-isomer), -66.8 (Major-isomer). 


\section{1-(3-Bromophenyl)-2,2,2-trifluoroethan-1-one 0-tosyl oxime k1}

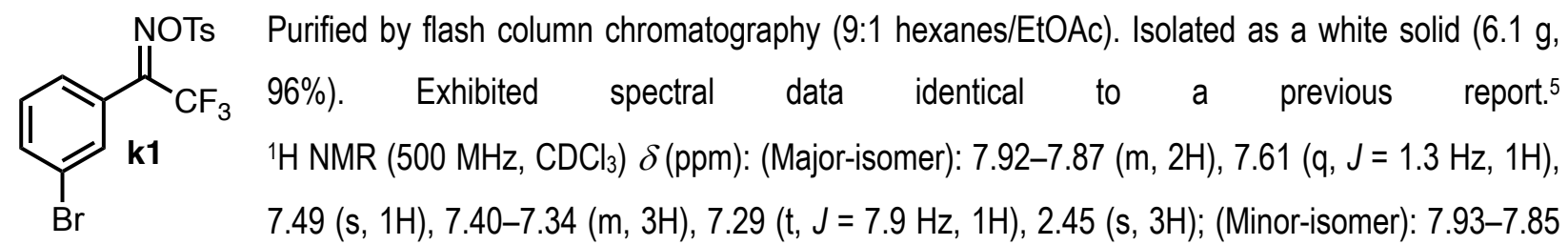
(m, 2H), $7.63(\mathrm{t}, \mathrm{J}=2.0 \mathrm{~Hz}, 1 \mathrm{H}), 7.54(\mathrm{~s}, 1 \mathrm{H}), 7.41-7.37(\mathrm{~m}, 3 \mathrm{H}), 7.36-7.30(\mathrm{~m}, 1 \mathrm{H}), 2.44(\mathrm{~s}, 3 \mathrm{H}) .{ }^{13} \mathrm{C}\left\{{ }^{1} \mathrm{H}\right\} \mathrm{NMR}(126$ $\left.\mathrm{MHz}, \mathrm{CDCl}_{3}\right) \delta(\mathrm{ppm}):($ Major-isomer): 152.6 (q, $J=33.9 \mathrm{~Hz}$ ), 146.6, 134.8, 131.6 (q, $J=1.4 \mathrm{~Hz}), 130.5,130.4,130.1$, 127.1, 122.8, 119.4 (q, $J=277.5 \mathrm{~Hz}$ ), 21.7; (Minor-isomer): 152.6 (q, $J=32.8 \mathrm{~Hz}$ ), 146.5, 134.9, 130.9, 130.3, 129.1, $127.5(q, J=1.4 \mathrm{~Hz}), 127.0,122.8,117.1$ (q, $J=283.8 \mathrm{~Hz}), 21.7 .{ }^{19} \mathrm{~F} \mathrm{NMR}\left(470 \mathrm{MHz}, \mathrm{CDCl}_{3}\right) \delta$ (ppm): -61.6 (Minorisomer), -66.9 (Major-isomer).

\section{2,2,2-Trifluoro-1-(4-(trifluoromethyl)phenyl)ethan-1-one 0-tosyl oxime I1}

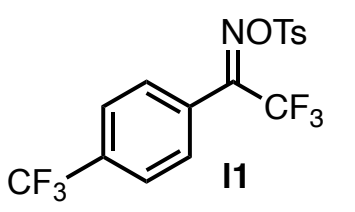

Purified by flash column chromatography (9:1 hexanes/EtOAc). Isolated as a white solid (1.9 g, 75\%). mp: $38-40{ }^{\circ} \mathrm{C} .{ }^{1} \mathrm{H}$ NMR (500 MHz, $\left.\mathrm{CDCl}_{3}\right) \delta$ (ppm): (Major-isomer): 7.94-7.91 (m, 2H), $7.76(\mathrm{~d}, J=8.1 \mathrm{~Hz}, 2 \mathrm{H}), 7.70(\mathrm{~d}, J=8.2 \mathrm{~Hz}, 2 \mathrm{H}), 7.42-7.40(\mathrm{~m}, 2 \mathrm{H}), 2.49$ (s, 3H); (Minor-isomer): 7.91-7.88 (m, 2H), $7.58(\mathrm{~d}, J=8.6 \mathrm{~Hz}, 2 \mathrm{H}), 7.51$ (d, J = 8.2 Hz, 2H), 7.39 $7.38(\mathrm{~m}, 2 \mathrm{H}), 2.47(\mathrm{~s}, 3 \mathrm{H}) \cdot{ }^{13} \mathrm{C}\left\{{ }^{1} \mathrm{H}\right\} \mathrm{NMR}\left(101 \mathrm{MHz}, \mathrm{CDCl}_{3}\right) \delta(\mathrm{ppm}):$ (Major-isomer only): 153.0 (q, J=34.2 Hz), 146.7, $133.5(q, J=33.1 \mathrm{~Hz}), 131.0,130.1,129.4,129.1,128.2,126.0$ (q, $J=3.8 \mathrm{~Hz}), 123.4(q, J=272.7 \mathrm{~Hz}), 119.5$ (q, $J=$ 277.4 Hz), 21.78. ${ }^{19} \mathrm{~F} \mathrm{NMR}\left(470 \mathrm{MHz}, \mathrm{CDCl}_{3}\right) \delta$ (ppm): (Minor-isomer): -61.5, -63.3; (Major-isomer): -63.3, -66.9. IR $\left(\mathrm{cm}^{-1}\right)$ : 2992, 2928, 1597, 1456, 1389, 1181, 893, 818. HR-MS (El) m/z [M + NH $\left.]_{4}\right]^{+}$calcd. for $\mathrm{C}_{16} \mathrm{H}_{15} \mathrm{~F}_{6} \mathrm{~N}_{2} \mathrm{O}_{3} \mathrm{~S} 429.0702$ found 429.0694 .

\section{2,2,2-Trifluoro-1-(2-methoxyphenyl)ethan-1-one 0-tosyl oxime m1}

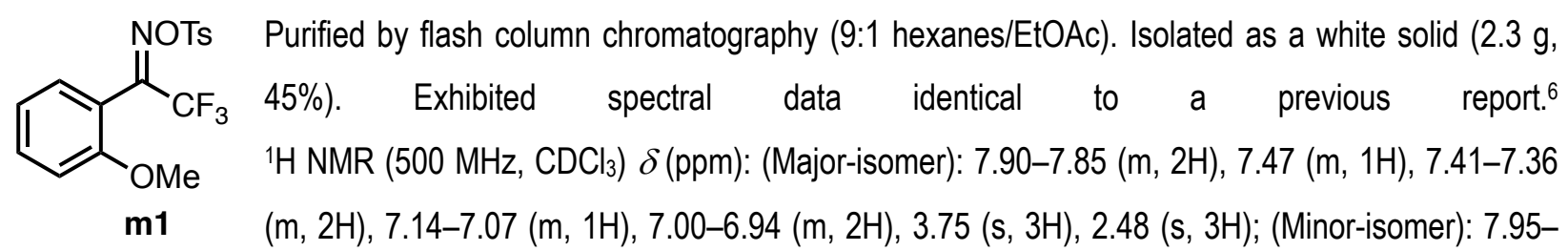
$7.80(\mathrm{~m}, 2 \mathrm{H}), 7.47$ (ddt, $\left.J^{1}=8.6 \mathrm{~Hz}, J^{2}=7.5 \mathrm{~Hz}, \boldsymbol{\beta}^{3}=1.9 \mathrm{~Hz}, 1 \mathrm{H}\right), 7.12-7.09(\mathrm{~m}, 2 \mathrm{H}), 7.02$ (dd, $J^{1}=7.5 \mathrm{~Hz}, J^{2}=0.9$ $\mathrm{Hz}, 1 \mathrm{H}), 6.92\left(\mathrm{dd}, \mathrm{J}^{1}=8.4, \mathrm{~J}^{2}=0.9 \mathrm{~Hz}, 2 \mathrm{H}\right), 3.78(\mathrm{~s}, 3 \mathrm{H}), 2.46(\mathrm{~s}, 3 \mathrm{H}) .{ }^{13} \mathrm{C}\left\{{ }^{1} \mathrm{H}\right\} \mathrm{NMR}\left(126 \mathrm{MHz}, \mathrm{CDCl}_{3}\right) \delta(\mathrm{ppm}):($ Majorisomer): 156.8, 153.0 (q, $J=34.7 \mathrm{~Hz}), 145.9,132.9,131.6,130.9,129.8,129.1,128.5,120.6,116.8$ (q, $J=282.8 \mathrm{~Hz})$, 111.5, 55.7, 21.8; (Minor-isomer): 158.5, 155.3 (q, J = 34.5 Hz), 145.9, 133.3, 131.6, 129.8, 129.6, 129.1, 128.8, 120.7, $119.4(\mathrm{q}, \mathrm{J}=276.9 \mathrm{~Hz}), 111.1,55.7,21.7 .{ }^{19} \mathrm{~F} \mathrm{NMR}\left(470 \mathrm{MHz}, \mathrm{CDCl}_{3}\right) \delta$ (ppm): -64.8 (Minor-isomer), -67.9 (Majorisomer). 


\section{2,2,2-Trifluoro-1-(2-fluorophenyl)ethan-1-one 0-tosyl oxime n1}

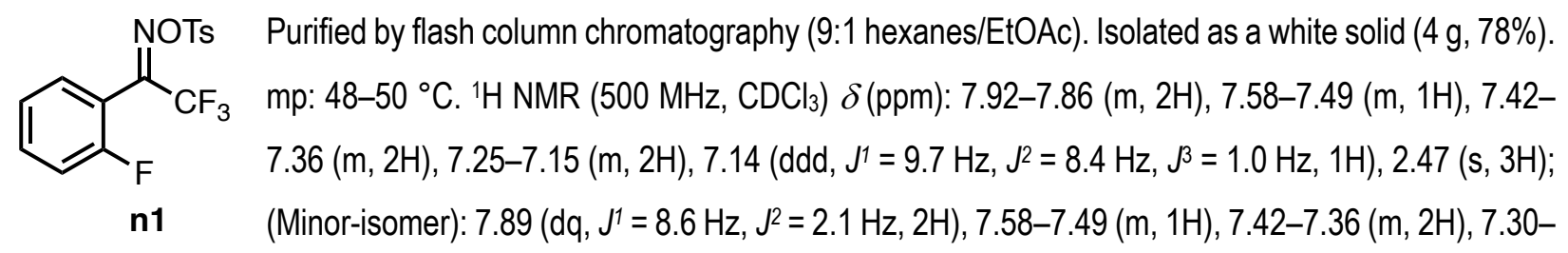

$7.22(\mathrm{~m}, 3 \mathrm{H}), 2.48(\mathrm{~s}, 3 \mathrm{H}) .{ }^{13} \mathrm{C}\left\{{ }^{1} \mathrm{H}\right\} \mathrm{NMR}\left(126 \mathrm{MHz}, \mathrm{CDCl}_{3}\right) \delta(\mathrm{ppm}):$ (Major-isomer): 160.6 (d, J = 253.1 Hz), 152.0 (q, $J=35.0 \mathrm{~Hz}$ ), 146.3, 133.9 (d, J = 8.2 Hz), 132.5 (d, J = 8.5 Hz), 131.2 (d, J = 1.1 Hz), 131.0 (q, J = 1.2 Hz), 130.0, 129.1, 124.6, 116.6 (q, $J=283.1 \mathrm{~Hz}$ ), 116.4 (d, $J=25.7 \mathrm{~Hz}$ ), 21.7; (Minor-isomer): 159.2 (d, $J=253.8 \mathrm{~Hz}$ ), 150.0 (q, $J$ $=35.3 \mathrm{~Hz}$ ), 146.3, 133.7 (d, $J=8.2 \mathrm{~Hz}), 132.4$ (d, $J=8.1 \mathrm{~Hz}), 129.9,129.2,128.8$ (d, $J=2.1 \mathrm{~Hz}), 124.6$ (d, $J=3.5$ $\mathrm{Hz}), 120.4$ (q, $J=276.7 \mathrm{~Hz}), 116.5,116.3,21.8 .{ }^{19} \mathrm{~F} \mathrm{NMR}\left(470 \mathrm{MHz}, \mathrm{CDCl}_{3}\right) \delta$ (ppm): (Major-isomer): -64.3 (d, $J=$ $10.0 \mathrm{~Hz}$ ), -109.9 (m); (Minor-isomer): -65.0 (d, J=8.5 Hz), -111.2 (m). IR ( $\left.\mathrm{cm}^{-1}\right): 2984,1562,1492,1389,1155,1092$, 893, 720. HR-MS (EI) m/z [M + NH $\left.]_{4}\right]^{+}$calcd. for $\mathrm{C}_{15} \mathrm{H}_{15} \mathrm{~F}_{4} \mathrm{~N}_{2} \mathrm{O}_{3} \mathrm{~S} 379.0734$ found 379.0722 .

\subsection{General procedure for the synthesis of 2,2,2-trifluoromethyl diaziridines}

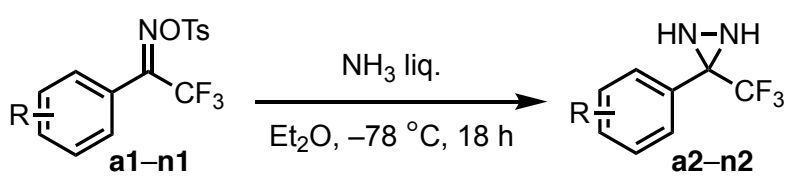

In a sealed microwave vial, the O-tosyl oxime $(2 \mathrm{mmol})$ was dissolved in $\mathrm{Et}_{2} \mathrm{O}(0.5 \mathrm{~mL})$ and cooled to $-78^{\circ} \mathrm{C}$. Anhydrous ammonia was condensed (ca. $10 \mathrm{~mL}$ ). The mixture was stirred overnight and brought up slowly to room temperature. The solution was cooled again to $-78^{\circ} \mathrm{C}$ and a bleed needle was added to remove excess pressure. The vial was then de-capped, and the residual ammonia was left to slowly evaporate at room temperature. The residue was diluted with $\mathrm{Et}_{2} \mathrm{O}$ and washed with $\mathrm{H}_{2} \mathrm{O}$, dried over $\mathrm{MgSO}_{4}$, and concentrated under reduced pressure to afford the corresponding diaziridine that was obtained clean and used as is for the oxidation into the diazirine.

\subsubsection{Characterizations of diaziridines a2-n2}

\section{3-(4,4'-Biphenyl)-3-(trifluoromethyl)diaziridine a2}<smiles>FC(F)(F)C1(c2ccc(-c3ccccc3)cc2)NN1</smiles>

Isolated as a white solid (530 $\mathrm{mg}$, quant.). Exhibited spectral data identical to a previous report. ${ }^{2}{ }^{1} \mathrm{H} \mathrm{NMR}\left(500 \mathrm{MHz}, \mathrm{CDCl}_{3}\right) \delta$ (ppm): 7.74-7.72 (m, 1H), 7.69-7.64 (m, 1H), 7.64-7.61 (m, 1H), 7.59-7.57 (m, 2H), 7.50-7.49 (m, 3H), 7.45-7.40 (m, 1H), $2.89(\mathrm{~d}, J=8.6 \mathrm{~Hz}, 1 \mathrm{H})$, $2.32(\mathrm{~d}, J=8.8 \mathrm{~Hz}, 1 \mathrm{H}) .{ }^{13} \mathrm{C}\left\{{ }^{1} \mathrm{H}\right\} \mathrm{NMR}\left(101 \mathrm{MHz}, \mathrm{CDCl}_{3}\right) \delta(\mathrm{ppm}): 131.9,129.0,129.0,128.8$, $127.5,127.2,127.0,123.6$ (q, $J=278.4 \mathrm{~Hz}), 121.6,57.9$ (q, $J=36.0 \mathrm{~Hz}) .{ }^{19} \mathrm{~F}$ NMR $\left(470 \mathrm{MHz}, \mathrm{CDCl}_{3}\right) \delta(\mathrm{ppm}):-75.3$. 


\section{3-(Naphthalin-2-yl)-3-(trifluoromethyl)diaziridine b2}

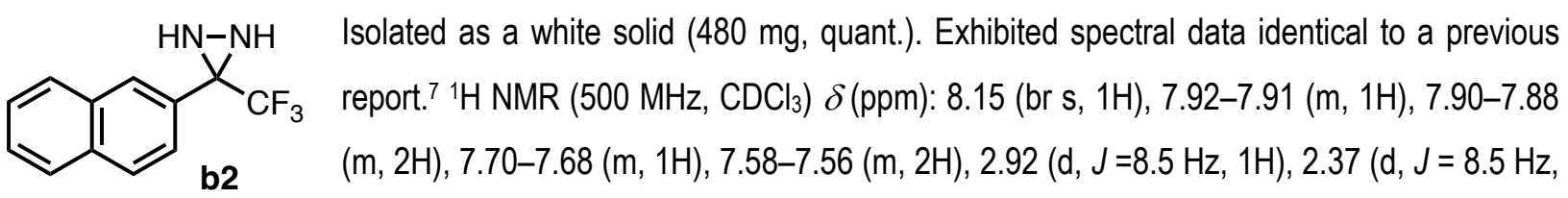

1H). ${ }^{13} \mathrm{C}\left\{{ }^{1} \mathrm{H}\right\} \operatorname{NMR}\left(126 \mathrm{MHz}, \mathrm{CDCl}_{3}\right) \delta(\mathrm{ppm}): 134.0,132.9,129.1,128.9,128.5,128.5,127.9,127.5,127.0,124.7$, $123.7(\mathrm{q}, \mathrm{J}=278.8 \mathrm{~Hz}), 58.4$ (q, $\mathrm{J}=36.1 \mathrm{~Hz}) .{ }^{19} \mathrm{~F} \mathrm{NMR}\left(470 \mathrm{MHz}, \mathrm{CDCl}_{3}\right) \delta(\mathrm{ppm}):-75.3$.

\section{3-(-4-Benzyloxyphenyl)-3-(trifluoromethyl)diaziridine c2}

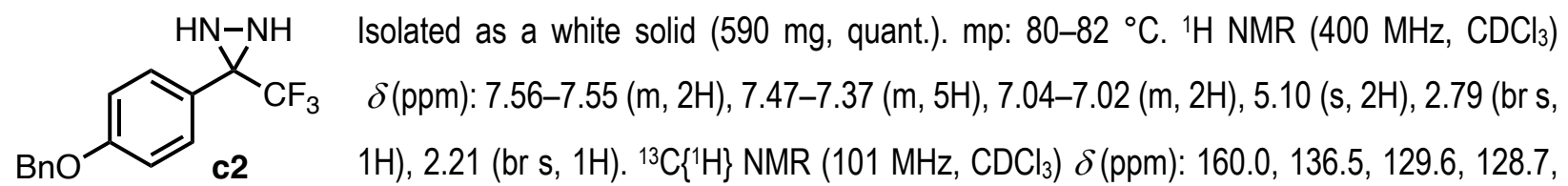
128.2, 127.5, 124.0, 123.7 (q, J = 278.2 Hz), 115.0, 70.1, 57.7 (q, $J=35.9 \mathrm{~Hz}) .{ }^{19} \mathrm{~F} \mathrm{NMR}\left(376 \mathrm{MHz}, \mathrm{CDCl}_{3}\right) \delta(\mathrm{ppm})$ : -75.7. IR (cm $\left.{ }^{-1}\right): 3200,2926,1614,1520,1014,743$. HR-MS (EI) m/z [M + H] $]^{+}$calcd. for $\mathrm{C}_{15} \mathrm{H}_{14} \mathrm{~F}_{3} \mathrm{~N}_{2} \mathrm{O} 295.1053$ found 295.1013.

\section{Bis-(4,4'-Biphenyl)-3-(trifluoromethyl)diaziridine d2}

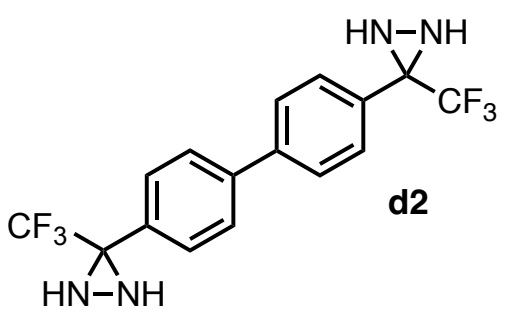

Isolated as a white solid (750 mg, quant.). mp: $150-152^{\circ} \mathrm{C} .{ }^{1} \mathrm{H}$ NMR $(500 \mathrm{MHz}$, $\left.\mathrm{CDCl}_{3}\right) \delta(\mathrm{ppm}): 7.74-7.72(\mathrm{~m}, 4 \mathrm{H}), 7.66-7.64(\mathrm{~m}, 4 \mathrm{H}), 2.85(\mathrm{br} \mathrm{s}, 2 \mathrm{H}), 2.26(\mathrm{br}$ s, $2 \mathrm{H}) .{ }^{13} \mathrm{C}\left\{{ }^{1} \mathrm{H}\right\} \operatorname{NMR}\left(126 \mathrm{MHz}, \mathrm{CDCl}_{3}\right) \delta(\mathrm{ppm}): 142.0,131.3,128.7,127.6$, $123.5(\mathrm{q}, J=278.1 \mathrm{~Hz}), 57.8$ (q, $J=36.2 \mathrm{~Hz}) .{ }^{19} \mathrm{~F} \mathrm{NMR}\left(470 \mathrm{MHz}, \mathrm{CDCl}_{3}\right)$ $\delta$ (ppm): -75.4. IR ( $\left.\mathrm{cm}^{-1}\right): 3211,2922,1381,1143,953,881,819,699 . \mathrm{HR}-\mathrm{MS}$

(El) $\mathrm{m} / \mathrm{z}[\mathrm{M}+\mathrm{H}]^{+}$calcd. for $\mathrm{C}_{16} \mathrm{H}_{13} \mathrm{~F}_{6} \mathrm{~N}_{4} 375.1039$ found 375.1041 .

\section{3-(p-Tolyl)-3-(trifluoromethyl)diaziridine e2}

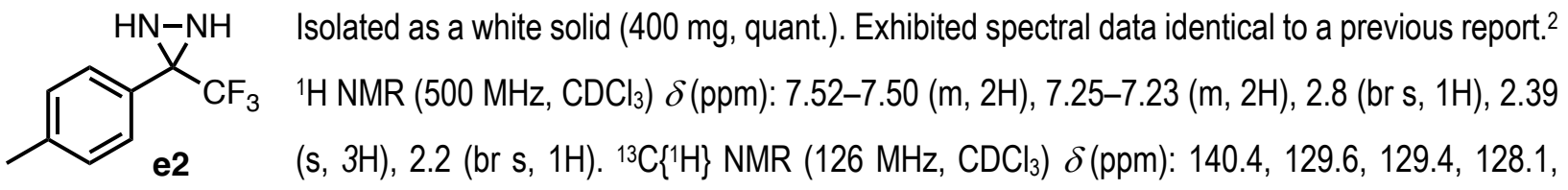
$123.7(\mathrm{q}, J=278.4 \mathrm{~Hz}), 58.1(\mathrm{q}, J=36.0 \mathrm{~Hz}), 21.4 .{ }^{19} \mathrm{~F} \mathrm{NMR}\left(470 \mathrm{MHz}, \mathrm{CDCl}_{3}\right) \delta(\mathrm{ppm}):-75.7$.

\section{3-(4-Tert-butylphenyl)-3-(trifluoromethyl)diaziridine f2}

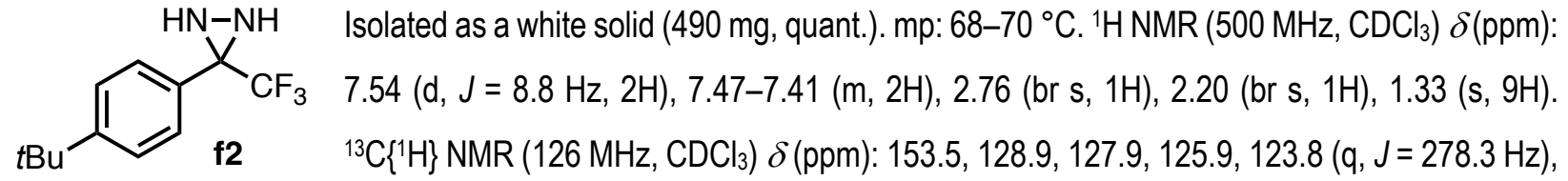
58.0 (q, J = 36.0 Hz), 34.9, 31.3. ${ }^{19} \mathrm{~F} \mathrm{NMR}\left(470 \mathrm{MHz}, \mathrm{CDCl}_{3}\right) \delta(\mathrm{ppm}):-75.6 . \mathrm{IR}\left(\mathrm{cm}^{-1}\right): 3248,3150,2966,1402,1142$, 829, 689. HR-MS (El) m/z [M + H] calcd. for $\mathrm{C}_{12} \mathrm{H}_{16} \mathrm{~F}_{3} \mathrm{~N}_{2} 245.1260$ found 245.1268. 


\section{3-(4-Methoxyphenyl)-3-(trifluoromethyl)diaziridine g2}

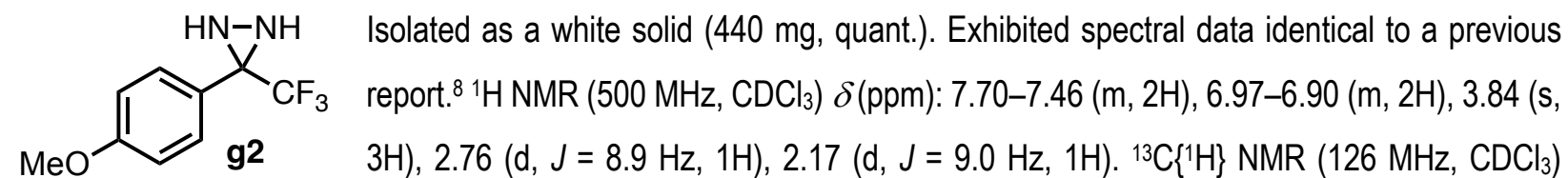

$\delta$ (ppm): $160.7,129.5,123.7,123.7$ (q, $J=278.2 \mathrm{~Hz}), 114.0,57.6$ (q, $J=35.9 \mathrm{~Hz}), 55.2 .{ }^{19} \mathrm{~F} \mathrm{NMR}\left(470 \mathrm{MHz}, \mathrm{CDCl}_{3}\right)$ $\delta(\mathrm{ppm}):-75.8$.

\section{3-Phenyl-3-(trifluoromethyl)diaziridine h2}

$\mathrm{HN}-\mathrm{NH} \quad$ Isolated as a white solid (380 mg, quant.). Exhibited spectral data identical to a previous report. ${ }^{9}$

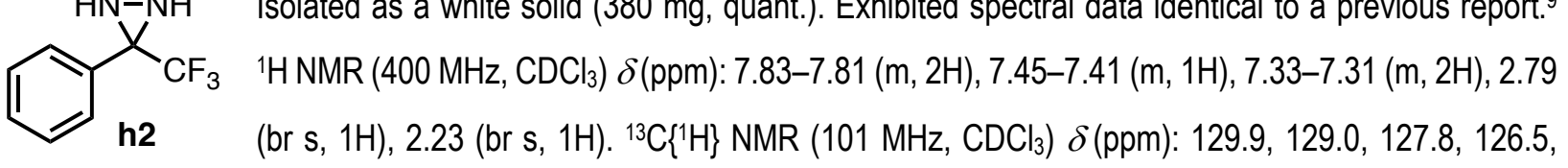
$123.7(\mathrm{q}, J=278.2 \mathrm{~Hz}), 58.3(\mathrm{q}, J=35.9 \mathrm{~Hz}) .{ }^{19} \mathrm{~F} \mathrm{NMR}\left(376 \mathrm{MHz}, \mathrm{CDCl}_{3}\right) \delta(\mathrm{ppm}):-75.6$.

\section{3-(4-Fluorophenyl)-3-(trifluoromethyl)diaziridine i2}

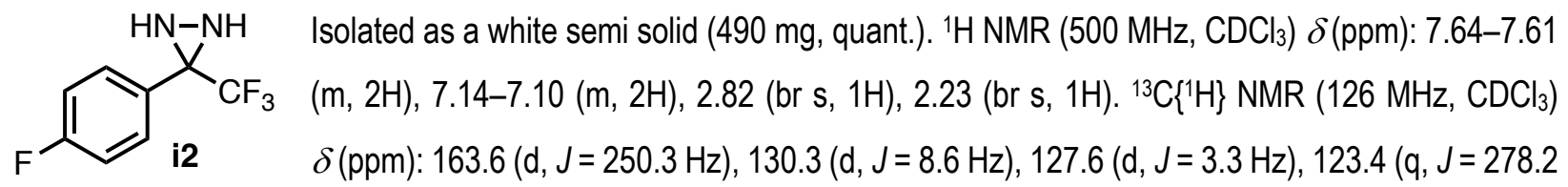
$\mathrm{Hz}), 115.9(\mathrm{~d}, J=21.9 \mathrm{~Hz}), 57.6(\mathrm{q}, J=36.2 \mathrm{~Hz}) .{ }^{19} \mathrm{~F} \mathrm{NMR}\left(470 \mathrm{MHz}, \mathrm{CDCl}_{3}\right) \delta(\mathrm{ppm}):-75.7,-110.1 . \mathrm{IR}\left(\mathrm{cm}^{-1}\right): 3256$, 3234, 1610, 1516, 1394, 1219, 1134, 1097, 951, 841. HR-MS (El) m/z [M + H] calcd. for $\mathrm{C}_{8} \mathrm{H}_{7} \mathrm{~F}_{4} \mathrm{~N}_{2} 207.0545$ found 207.0547

\section{3-(4-Bromophenyl)-3-(trifluoromethyl)diaziridine j2}<smiles>FC(F)(F)C1(c2ccc(Br)cc2)NN1</smiles>
Isolated as a white semi solid (540 mg, quant.). Exhibited spectral data identical to a previous report. ${ }^{4}{ }^{1} \mathrm{H}$ NMR $\left(500 \mathrm{MHz}, \mathrm{CDCl}_{3}\right) \delta$ (ppm): 7.59-7.57 (m, 2H), 7.52-7.50 (m, 2H), 2.82 (br s, 1H), 2.21 (br s, $1 \mathrm{H}) .{ }^{13} \mathrm{C}\left\{{ }^{1} \mathrm{H}\right\}$ NMR $\left(126 \mathrm{MHz}, \mathrm{CDCl}_{3}\right) \delta$ (ppm): 132.2, 130.8, 129.9, 124.8, 123.4 (q, $J=278.2 \mathrm{~Hz}), 57.8$ (q, $J=36.3 \mathrm{~Hz}) .{ }^{19} \mathrm{~F} \mathrm{NMR}\left(470 \mathrm{MHz}, \mathrm{CDCl}_{3}\right) \delta(\mathrm{ppm}):-75.5$.

\section{3-(3-Bromophenyl)-3-(trifluoromethyl)diaziridine k2}

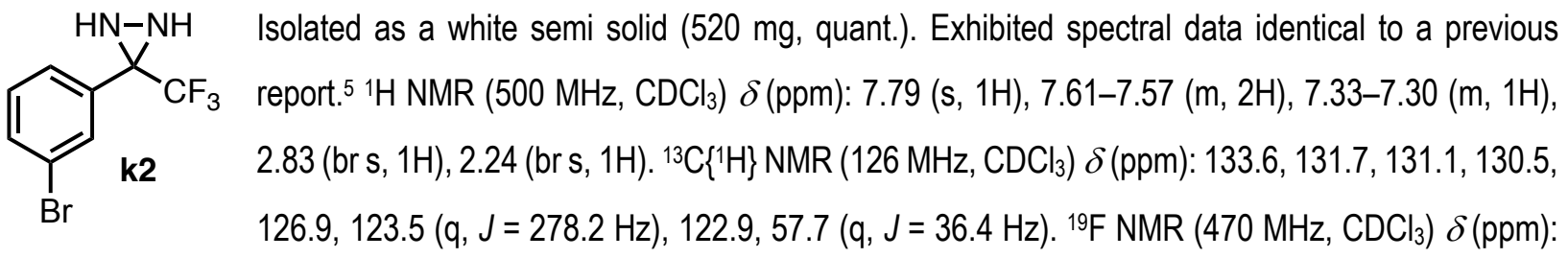
$-75.3$. 


\section{3-(4-Trifluoromethylphenyl)-3-(trifluoromethyl)diaziridine 12}

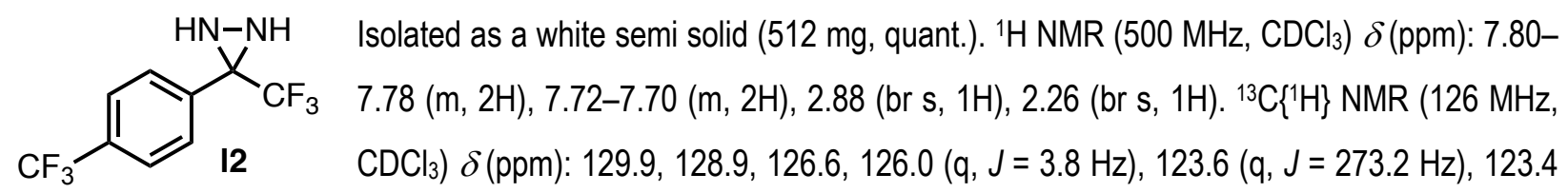

$(q, J=278.4 \mathrm{~Hz}), 57.8(\mathrm{q}, J=35.6 \mathrm{~Hz}) .{ }^{19} \mathrm{~F} \mathrm{NMR}\left(470 \mathrm{MHz}, \mathrm{CDCl}_{3}\right) \delta(\mathrm{ppm}):-63.0,-75.2 . \mathrm{IR}\left(\mathrm{cm}^{-1}\right): 3356,3259,2962$, 1599, 1526, 1325, 1128, 1067, 1018, 951, 844, 712. HR-MS (EI) m/z [M + Na] $]^{+}$calcd. for $\mathrm{C}_{9} \mathrm{H}_{6} \mathrm{~F}_{6} \mathrm{~N}_{2} \mathrm{Na} 279.0327$ found 279.0921.

\section{3-(2-Methoxyphenyl)-3-(trifluoromethyl)diaziridine $\mathrm{m} 2$}

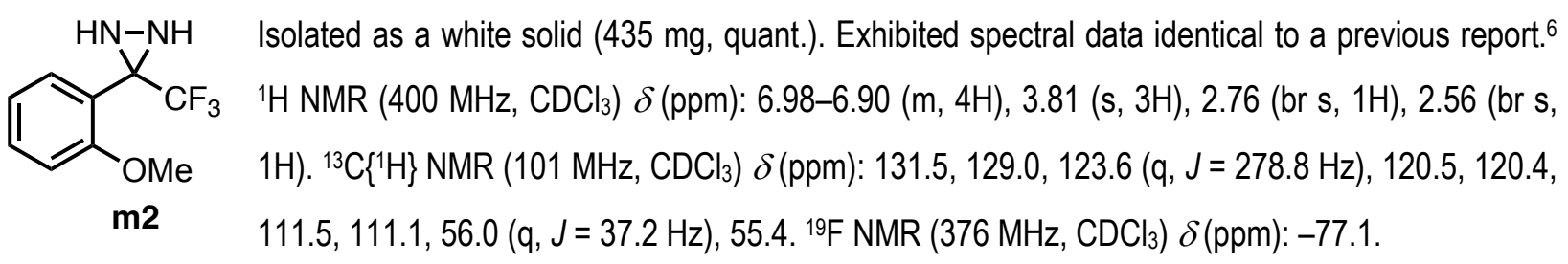

\section{3-(2-Fluorophenyl)-3-(trifluoromethyl)diaziridine n2}

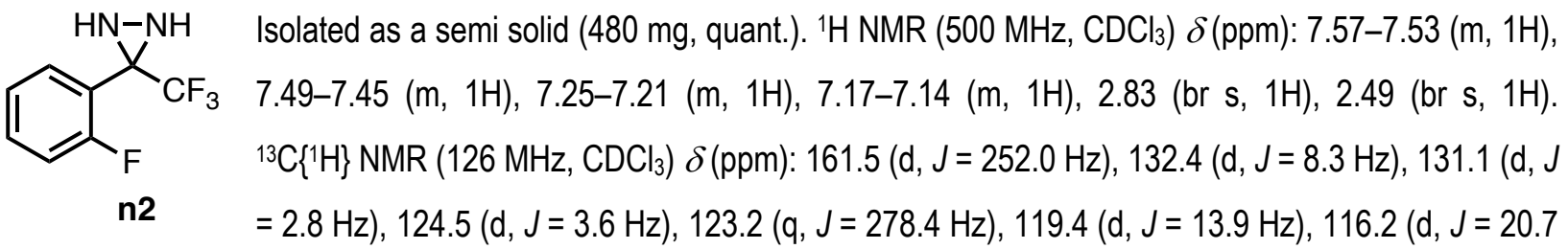
$\mathrm{Hz}), 55.5(\mathrm{q}, J=38.0 \mathrm{~Hz}) .{ }^{19} \mathrm{~F} \mathrm{NMR}\left(470 \mathrm{MHz}, \mathrm{CDCl}_{3}\right) \delta(\mathrm{ppm}):-77.4,-114.1 . \mathrm{IR}\left(\mathrm{cm}^{-1}\right): 3259,1620,1499,1230$, 1142, 949, 758. HR-MS (EI) m/z [M + H] calcd. for $\mathrm{C}_{8} \mathrm{H}_{7} \mathrm{~F}_{4} \mathrm{~N}_{2} 207.0545$ found 207.0547.

\subsection{Procedure for synthesis of 2,2,2-trifluoromethyl diazirines $1 a-n$}

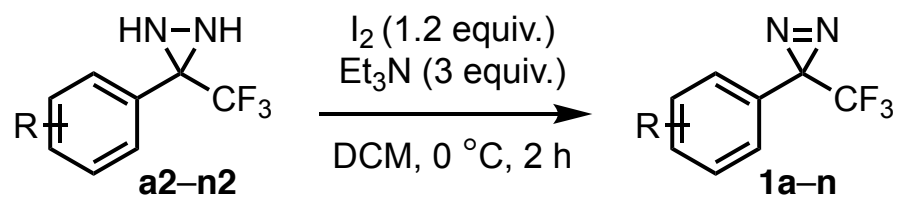

To a solution of the diaziridine $(30 \mathrm{mmol})$ in $\mathrm{CH}_{2} \mathrm{Cl}_{2}(50 \mathrm{~mL})$ at $0{ }^{\circ} \mathrm{C}, \mathrm{I}_{2}(1.4 \mathrm{~g}, 36 \mathrm{mmol})$ and $\mathrm{Et}_{3} \mathrm{~N}(8.4 \mathrm{~mL}, 60 \mathrm{mmol})$ were added. The solution was stirred for $1 \mathrm{~h}$ and the excess of $\mathrm{I}_{2}$ was quenched with $1 \mathrm{M} \mathrm{NaOH}_{\text {aq. The mixture was }}$ extracted with $\mathrm{CH}_{2} \mathrm{Cl}_{2}$, dried over $\mathrm{MgSO}_{4}$, and concentrated under reduced pressure. The residue was purified by flash column chromatography (Biotage, SNAP ULTRA, 100\% hexanes) to give the desired diazirine 17. 


\subsubsection{Characterizations of 2,2,2-trifluoromethyl diazirines 1a-n}

\section{3-([1,1'-Biphenyl]-4-yl)-3-(trifluoromethyl)-3H-diazirine 1a}

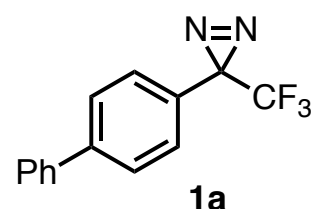

Purified by flash column chromatography (100\% hexanes). Isolated as a white solid ( $6.5 \mathrm{~g}$, $81 \%)$. Exhibited spectral data identical to a previous report. ${ }^{2}{ }^{1} \mathrm{H} \mathrm{NMR}\left(500 \mathrm{MHz}, \mathrm{CDCl}_{3}\right) \delta$ (ppm): 7.69-7.65 (m, 4H), 7.57-7.49 (m, 3H), 7.39-7.36 (m, 2H). ${ }^{13} \mathrm{C}\left\{{ }^{1} \mathrm{H}\right\}$ NMR $(126 \mathrm{MHz}$, $\left.\mathrm{CDCl}_{3}\right) \delta(\mathrm{ppm}): 142.7,139.9,129.1,128.1,128.1,127.6,127.2,127.0,122.4$ (q, J = 274.7

$\mathrm{Hz}), 28.1$ (q, $J=39.8 \mathrm{~Hz}) .{ }^{19} \mathrm{~F} \mathrm{NMR}\left(470 \mathrm{MHz}, \mathrm{CDCl}_{3}\right) \delta(\mathrm{ppm}):-65.0$.

\section{3-(Naphthalen-2-yl)-3-(trifluoromethyl)-3H-diazirine 1b}

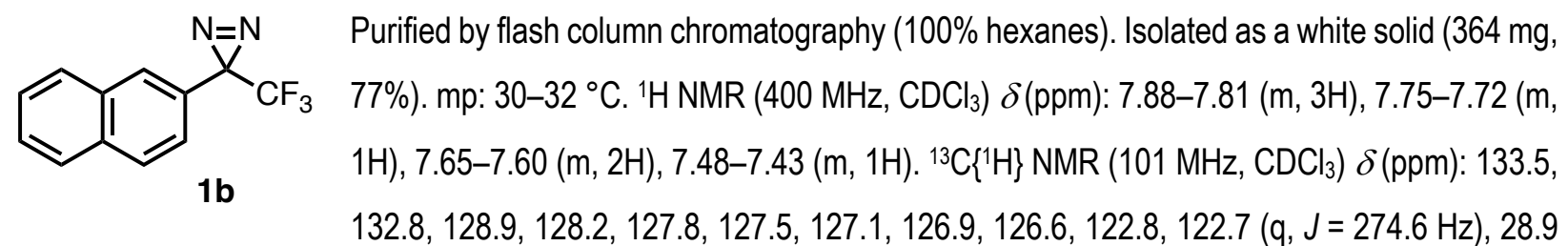

(q, J = 40.3 Hz). $\left.{ }^{19} \mathrm{~F} \mathrm{NMR} \mathrm{(376} \mathrm{MHz,} \mathrm{CDCl}_{3}\right) \delta(\mathrm{ppm}):-64.9 . \mathrm{IR}\left(\mathrm{cm}^{-1}\right): 3065,1622,1599,1323,1149,991,816,750$. HR-MS (El) $\mathrm{m} / \mathrm{z}\left[\mathrm{M}+\mathrm{NH}_{4}\right]^{+}$calcd. for $\mathrm{C}_{12} \mathrm{H}_{13} \mathrm{~F}_{3} \mathrm{~N} 228.0995$ found 228.1045.

\section{3-(4-(Benzyloxy)phenyl)-3-(trifluoromethyl)-3H-diazirine 1c}<smiles>FC(F)(F)C1(c2ccc(OBr)cc2)N=N1</smiles>

$1 \mathrm{c}$

Purified by flash column chromatography (100\% hexanes). Isolated as an off-white solid (371 mg, 63\%). mp: 38-40 ㄷ. ${ }^{1} \mathrm{H} \mathrm{NMR}\left(400 \mathrm{MHz}, \mathrm{CDCl}_{3}\right) \delta$ (ppm): 7.49-7.44 (m, 4H), 7.42$7.39(\mathrm{~m}, 1 \mathrm{H}), 7.23-7.21(\mathrm{~m}, 2 \mathrm{H}), 7.05-7.03(\mathrm{~m}, 2 \mathrm{H}), 5.11(\mathrm{~s}, 2 \mathrm{H}) .{ }^{13} \mathrm{C}\left\{{ }^{1} \mathrm{H}\right\} \mathrm{NMR}(101 \mathrm{MHz}$, $\left.\mathrm{CDCl}_{3}\right) \delta(\mathrm{ppm}): 159.9,136.5,128.8,128.3,128.3,127.6,122.4$ (q, $\left.J=274.6 \mathrm{~Hz}\right), 121.3$,

115.4, 70.2, 28.4 (q, J = 40.6 Hz). $\left.{ }^{19} \mathrm{~F} \mathrm{NMR} \mathrm{(376} \mathrm{MHz,} \mathrm{CDCl}_{3}\right) \delta(\mathrm{ppm}):-65.5 . \mathrm{IR}\left(\mathrm{cm}^{-1}\right): 3039,1614,1518,1148$, 1011, 696. HR-MS (EI) $\mathrm{m} / \mathrm{z}[\mathrm{M}+\mathrm{H}]^{+}$calcd. for $\mathrm{C}_{15} \mathrm{H}_{12} \mathrm{~F}_{3} \mathrm{O} 265.0840$ found 265.0411 .

\section{4,4'-Bis(3-(trifluoromethyl)-3H-diazirin-3-yl)-1,1'-biphenyl 1d}<smiles>FC(F)(F)C1(c2ccc(-c3ccc(C4(C(F)(F)F)N=N4)cc3)cc2)N=N1</smiles>

Double the equivalents to those reported in the general procedures were used to make the bis-diazirine. Purified by flash column chromatography $(100 \%$ hexanes). Isolated as a yellow solid (461 mg, 62\%). mp: $44-46{ }^{\circ} \mathrm{C}$. ${ }^{1} \mathrm{H}$ NMR (400 MHz, $\left.\mathrm{CDCl}_{3}\right) \delta(\mathrm{ppm}): 7.61-7.59(\mathrm{~m}, 2 \mathrm{H}), 7.33-7.30(\mathrm{~m}, 2 \mathrm{H}) \cdot{ }^{13} \mathrm{C}\left\{{ }^{1} \mathrm{H}\right\} \mathrm{NMR}$ $\left(101 \mathrm{MHz}, \mathrm{CDCl}_{3}\right) \delta(\mathrm{ppm}): 140.2,128.1,126.6,126.2,121.4$ (q, J = 274.6 Hz),

27.6 (q, $J=40.5 \mathrm{~Hz}) .{ }^{19} \mathrm{~F} \mathrm{NMR}\left(376 \mathrm{MHz}, \mathrm{CDCl}_{3}\right) \delta(\mathrm{ppm}):-65.3 . \mathrm{IR}\left(\mathrm{cm}^{-1}\right): 3047,1917,1609,1342,1159,935,808$. HR-MS (El) m/z [M + H] $]^{+}$calcd. for $\mathrm{C}_{16} \mathrm{H}_{9} \mathrm{~F}_{6} 314.0608$ found 2314.0648 . 


\section{3-(p-Tolyl)-3-(trifluoromethyl)-3H-diazirine 1e}

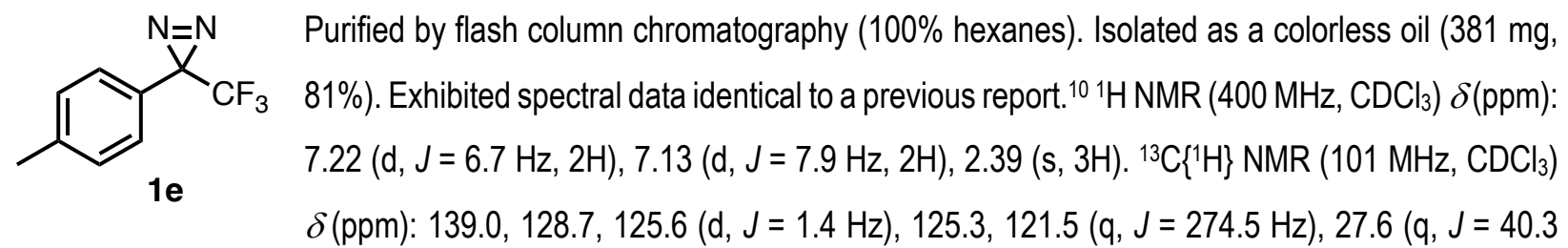

$\mathrm{Hz}), 20.3 .{ }^{19} \mathrm{~F} \mathrm{NMR}\left(376 \mathrm{MHz}, \mathrm{CDCl}_{3}\right) \delta(\mathrm{ppm}):-65.5$.

\section{3-(4-(Tert-butyl)phenyl)-3-(trifluoromethyl)-3H-diazirine $1 f$}<smiles>CC(C)(C)c1ccc(C2(C(F)(F)F)N=N2)cc1</smiles>

$1 f$

Purified by flash column chromatography (100\% hexanes). Isolated as a colorless oil (869 $\mathrm{mg}, 80 \%) .{ }^{1} \mathrm{H}$ NMR $\left(400 \mathrm{MHz}, \mathrm{CDCl}_{3}\right) \delta(\mathrm{ppm}): 7.52-7.49(\mathrm{~m}, 2 \mathrm{H}), 7.24-7.22(\mathrm{~m}, 2 \mathrm{H}), 1.39$ (s, 9H). ${ }^{13} \mathrm{C}\left\{{ }^{1} \mathrm{H}\right\} \mathrm{NMR}\left(101 \mathrm{MHz}, \mathrm{CDCl}_{3}\right) \delta$ (ppm): 152.2, 125.5, 125.4, 125.0, 121.5 (q, $\mathrm{J}=$ $274.5 \mathrm{~Hz}$ ), 33.9, 30.2, 27.6 (q, J = 40.3 Hz). $\left.{ }^{19} \mathrm{~F} \mathrm{NMR} \mathrm{(376} \mathrm{MHz,} \mathrm{CDCl}_{3}\right) \delta(\mathrm{ppm}):-65.4$. IR (cm-1): 2966, 1618, 1344, 1182, 1151, 939, 702. HR-MS (El) m/z [M + H] calcd. for $\mathrm{C}_{12} \mathrm{H}_{14} \mathrm{~F}_{3} 215.1048$ found 215.1028.

\section{3-(4-Methoxyphenyl)-3-(trifluoromethyl)-3H-diazirine $1 \mathrm{~g}$}<smiles>COc1ccc(C2(C(F)(F)F)N=N2)cc1</smiles>

$1 \mathrm{~g}$

Purified by flash column chromatography (100\% hexanes). Isolated as a colorless oil (322 $\mathrm{mg}, 75 \%)$. Exhibited spectral data identical to a previous report. ${ }^{10}{ }^{1} \mathrm{H} \mathrm{NMR}\left(500 \mathrm{MHz}, \mathrm{CDCl}_{3}\right)$ $\delta$ (ppm): 7.16 (d, J = $8.3 \mathrm{~Hz}, 2 \mathrm{H}), 6.92\left(\mathrm{dt}, \mathrm{J}^{1}=8.9 \mathrm{~Hz}, \mathrm{~J}^{2}=3.0 \mathrm{~Hz}, J^{3}=2.2 \mathrm{~Hz}, 2 \mathrm{H}\right.$ ), 3.82 (s, 3H). ${ }^{13} \mathrm{C}\left\{{ }^{1} \mathrm{H}\right\} \mathrm{NMR}\left(126 \mathrm{MHz}, \mathrm{CDCl}_{3}\right) \delta(\mathrm{ppm}): 160.7,128.3,123.4$ (q, J=273.9 Hz), 121.1, 114.5, 55.5, 28.3 (q, J = 40.4 Hz). $\left.{ }^{19} \mathrm{~F} \mathrm{NMR} \mathrm{(470} \mathrm{MHz,} \mathrm{CDCl}_{3}\right) \delta(\mathrm{ppm}):-65.6$.

\section{3-Phenyl-3-(trifluoromethyl)-3H-diazirine $1 \mathrm{~h}$}<smiles>FC(F)(F)C1(c2ccccc2)N=N1</smiles>

$1 \mathrm{~h}$

Purified by flash column chromatography (100\% hexanes). Isolated as a colorless oil (123 mg, $50 \%$ ). Exhibited spectral data identical to a previous report. ${ }^{10}{ }^{1} \mathrm{H} \mathrm{NMR}\left(400 \mathrm{MHz}, \mathrm{CDCl}_{3}\right) \delta(\mathrm{ppm})$ : 7.43-7.41 (m, 3H), 7.24-7.22 (m, 2H). ${ }^{13} \mathrm{C}\left\{{ }^{1} \mathrm{H}\right\}$ NMR (101 MHz, $\left.\mathrm{CDCl}_{3}\right) \delta(\mathrm{ppm}): 130.9,129.6$, 128.8, 126.4, 122.2 (q, $J=274.6 \mathrm{~Hz}), 28.4(\mathrm{q}, J=40.4 \mathrm{~Hz}) .{ }^{19} \mathrm{~F} \mathrm{NMR}\left(376 \mathrm{MHz}, \mathrm{CDCl}_{3}\right) \delta(\mathrm{ppm}):-$

65.4.

\section{3-(4-Fluorophenyl)-3-(trifluoromethyl)-3H-diazirine 1i}<smiles>Fc1ccc(C2(C(F)(F)F)N=N2)cc1</smiles>

$1 \mathrm{i}$

Purified by flash column chromatography (100\% hexanes). Isolated as a volatile colorless oil (74 mg, 20\%). ${ }^{1} \mathrm{H}$ NMR (400 MHz, $\left.\mathrm{CDCl}_{3}\right) \delta(\mathrm{ppm}):$ 7.24-7.21 (m, 2H), 7.11-7.07 (m, 2H). ${ }^{13} \mathrm{C}\left\{{ }^{1} \mathrm{H}\right\} \mathrm{NMR}\left(101 \mathrm{MHz}, \mathrm{CDCl}_{3}\right) \delta(\mathrm{ppm}): 162.6(\mathrm{~d}, J=250.8 \mathrm{~Hz}), 127.9(\mathrm{~d}, J=8.7 \mathrm{~Hz}), 124.1$ (d, $J=3.1 \mathrm{~Hz}), 121.3$ (q, $J=274.5 \mathrm{~Hz}), 115.3$ (q, $J=22.2 \mathrm{~Hz}), 27.3$ (q, $J=40.7 \mathrm{~Hz}) .{ }^{19} \mathrm{~F} \mathrm{NMR}$ 
(376 MHz, $\left.\mathrm{CDCl}_{3}\right) \delta(\mathrm{ppm}):-65.8,-110.2 . \mathrm{IR}\left(\mathrm{cm}^{-1}\right): 3087,1614,1516,1199,937,825 . \mathrm{HR}-\mathrm{MS}$ (El) m/z [M + H] ${ }^{+}$ calcd. for $\mathrm{C}_{8} \mathrm{H}_{5} \mathrm{~F}_{4} 177.0327$ found 177.0319 .

\section{3-(4-Bromophenyl)-3-(trifluoromethyl)-3H-diazirine 1j}

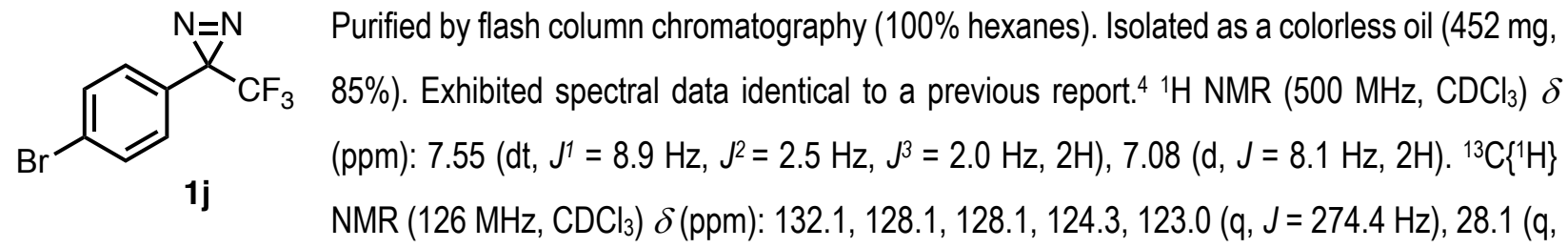
$J=41.0 \mathrm{~Hz}) .{ }^{19} \mathrm{~F} \mathrm{NMR}\left(470 \mathrm{MHz}, \mathrm{CDCl}_{3}\right) \delta(\mathrm{ppm}):-65.3$.

\section{3-(3-Bromophenyl)-3-(trifluoromethyl)-3H-diazirine $1 \mathrm{k}$}

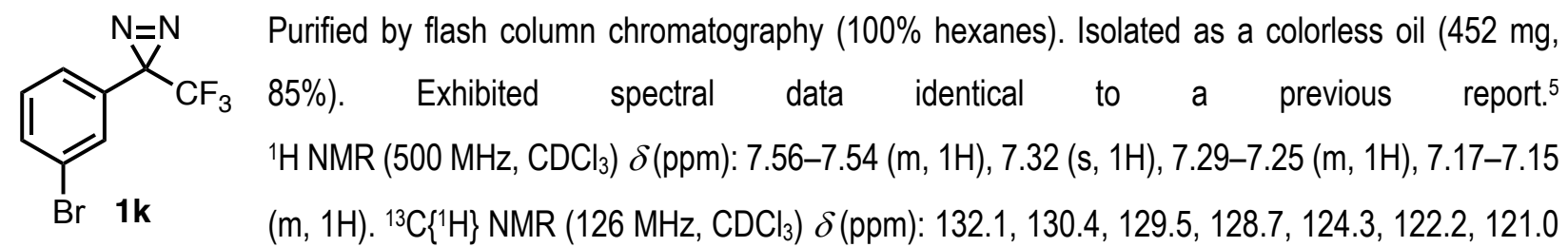

(q, $J=276.4 \mathrm{~Hz}), 27.1$ (q, $J=41.0 \mathrm{~Hz}) .{ }^{19} \mathrm{~F} \mathrm{NMR}\left(376 \mathrm{MHz}, \mathrm{CDCl}_{3}\right) \delta(\mathrm{ppm}):-65.2$.

\section{3-(Trifluoromethyl)-3-(4-(trifluoromethyl)phenyl)-3H-diazirine 1 I}<smiles>FC(F)(F)c1ccc(C2(C(F)(F)F)N=N2)cc1</smiles>

11

Purified by flash column chromatography (100\% hexanes). Isolated as a colorless oil (401 mg, 79\%). ${ }^{1} \mathrm{H}$ NMR (500 MHz, $\left.\mathrm{CDCl}_{3}\right) \delta$ (ppm): 7.68-7.66 (m, 2H), 7.33-7.31 (m, 2H). ${ }^{13} \mathrm{C}\left\{{ }^{1} \mathrm{H}\right\} \operatorname{NMR}\left(126 \mathrm{MHz}, \mathrm{CDCl}_{3}\right) \delta(\mathrm{ppm}): 132.1$ (q, $\left.J=1.7 \mathrm{~Hz}\right), 131.0$ (q, $\left.J=33.2 \mathrm{~Hz}\right), 126.0$ (q, J = $1.8 \mathrm{~Hz}$ ), 125.0 (q, J = 3.7 Hz), 120.9 (q, J=273.0 Hz), 120.5 (q, J=275.4 Hz), 27.4 (q, J = 40.9 Hz). ${ }^{19} \mathrm{~F} \mathrm{NMR}\left(470 \mathrm{MHz}, \mathrm{CDCl}_{3}\right) \delta(\mathrm{ppm}):-63.1,-65.0 . \mathrm{IR}\left(\mathrm{cm}^{-1}\right): 2958,1622,1325,1124,939,829 . \mathrm{HR}-$ $\mathrm{MS}(\mathrm{El}) \mathrm{m} / \mathrm{z}[\mathrm{M}+\mathrm{K}]^{+}$calcd. for $\mathrm{C}_{9} \mathrm{H}_{4} \mathrm{~F}_{6} \mathrm{~K} 264.9854$ found 264.9484 .

\section{3-(2-Methoxyphenyl)-3-(trifluoromethyl)-3H-diazirine $1 \mathrm{~m}$}

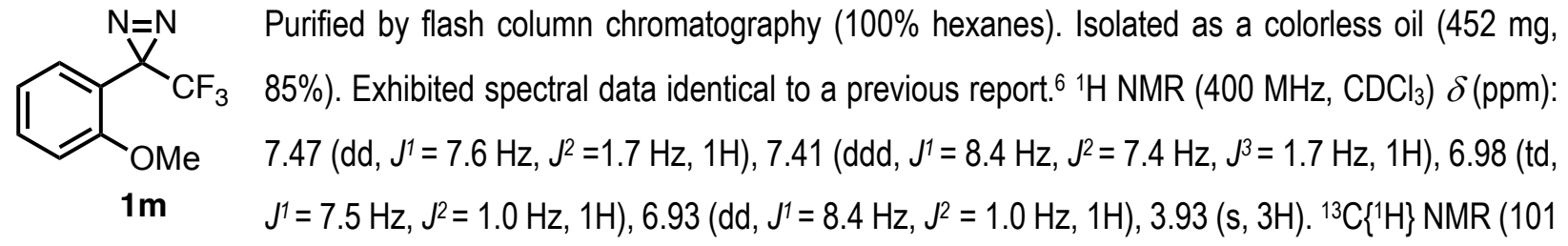
$\left.\mathrm{MHz}, \mathrm{CDCl}_{3}\right) \delta(\mathrm{ppm}): 159.1,131.3,130.1,127.0$ (q, $\left.\mathrm{J}=288.1 \mathrm{~Hz}\right), 120.1,115.8,110.7,54.8,25.6$ (q, $\left.J=42.6 \mathrm{~Hz}\right)$. ${ }^{19} \mathrm{~F} \mathrm{NMR}\left(470 \mathrm{MHz}, \mathrm{CDCl}_{3}\right) \delta(\mathrm{ppm}):-69.3$. 


\section{3-(2-Fluorophenyl)-3-(trifluoromethyl)-3H-diazirine 1n}

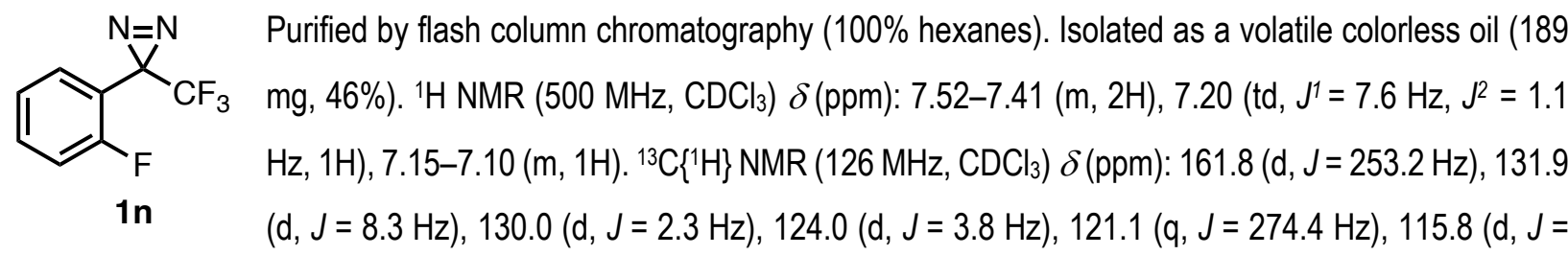

$20.7 \mathrm{~Hz}), 115.1$ (d, $J=14.5 \mathrm{~Hz}), 24.6$ (q, $J=43.1 \mathrm{~Hz}) .{ }^{19} \mathrm{~F} \mathrm{NMR}\left(470 \mathrm{MHz}, \mathrm{CDCl}_{3}\right) \delta(\mathrm{ppm}):-68.9,-113.0 . \mathrm{IR}\left(\mathrm{cm}^{-1}\right)$ : 3074, 1624, 1499, 1186, 1153, 941, 758. HR-MS (EI) m/z [M + H] $]^{+}$calcd. for $\mathrm{C}_{8} \mathrm{H}_{5} \mathrm{~F}_{4} 177.0327$ found 177.0318. 


\section{UV-Vis spectra}

\subsection{UV-Vis spectra of diazirines $1 \mathrm{a}-1 \mathrm{n}$}
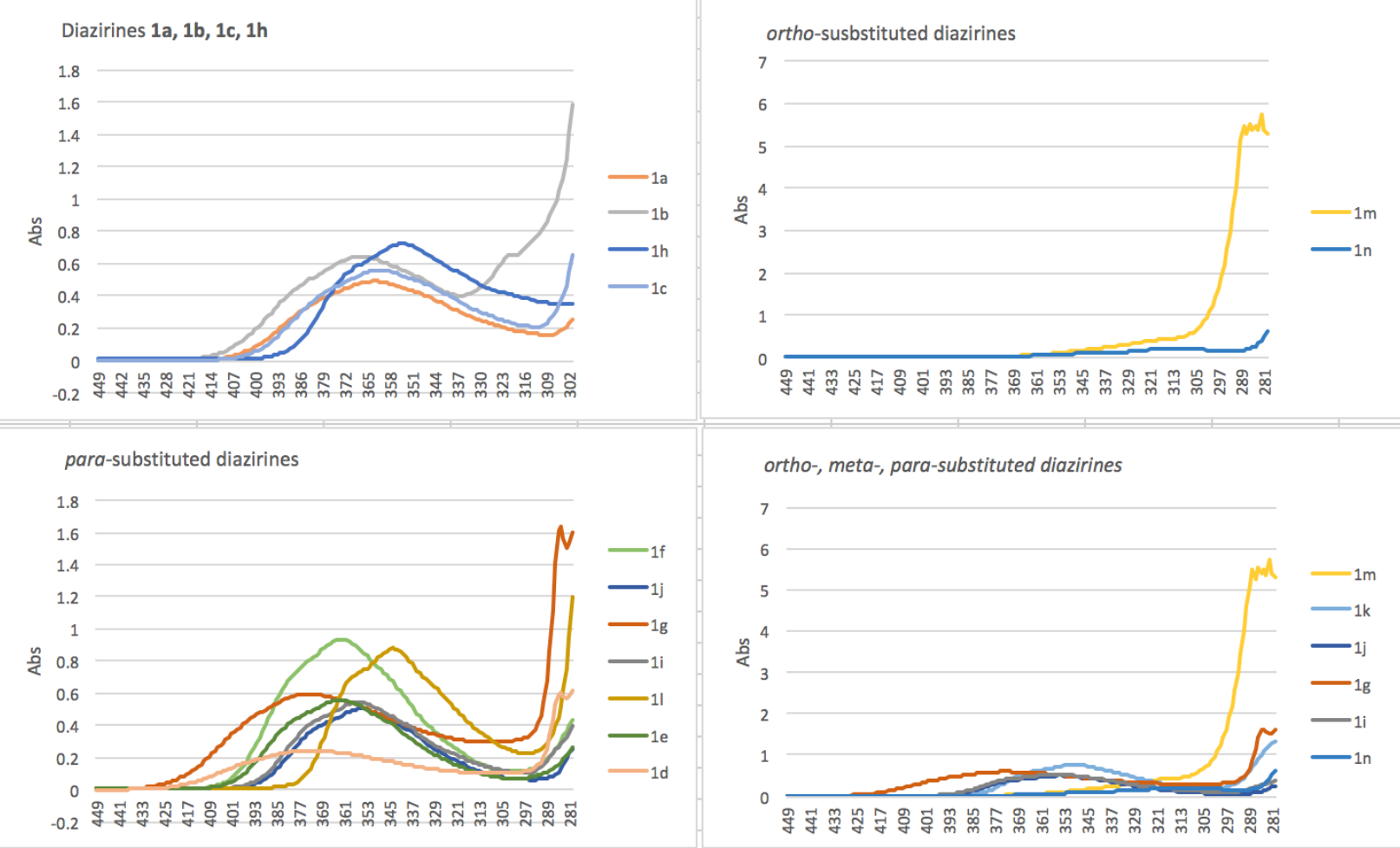<smiles>FC(F)(F)C1(c2ccc(-c3ccccc3)cc2)N=N1</smiles>

$1 a$<smiles>FC(F)(F)C1(c2ccc3ccccc3c2)N=N1</smiles>

$1 b$<smiles>FC(F)(F)c1ccc(C2(C(F)(F)F)N=N2)cc1</smiles>

$1 f$<smiles>FC(F)(F)C1(c2cccc(Br)c2)N=N1</smiles><smiles>COc1ccc(C2(C(F)(F)F)N=N2)cc1</smiles><smiles>FC(F)(F)c1ccc(C2(C(F)(F)F)N=N2)cc1</smiles><smiles>O=C(Oc1ccc(C2(C(F)(F)F)N=N2)cc1)c1ccccc1</smiles><smiles>FC(F)(F)C1(c2ccc(-c3ccc(C4(C(F)(F)F)N=N4)cc3)cc2)N=N1</smiles><smiles>Cc1ccc(C2(C(F)(F)F)N=N2)cc1</smiles><smiles>FC(F)(F)C1(c2ccc(Br)cc2)N=N1</smiles>

$1 \mathrm{~h}$<smiles>COc1ccccc1C1(C(F)(F)F)N=N1</smiles><smiles>Fc1ccccc1C1(C(F)(F)F)N=N1</smiles>

1n 


\subsection{UV-Vis spectra of cyclopropene $2 a$}

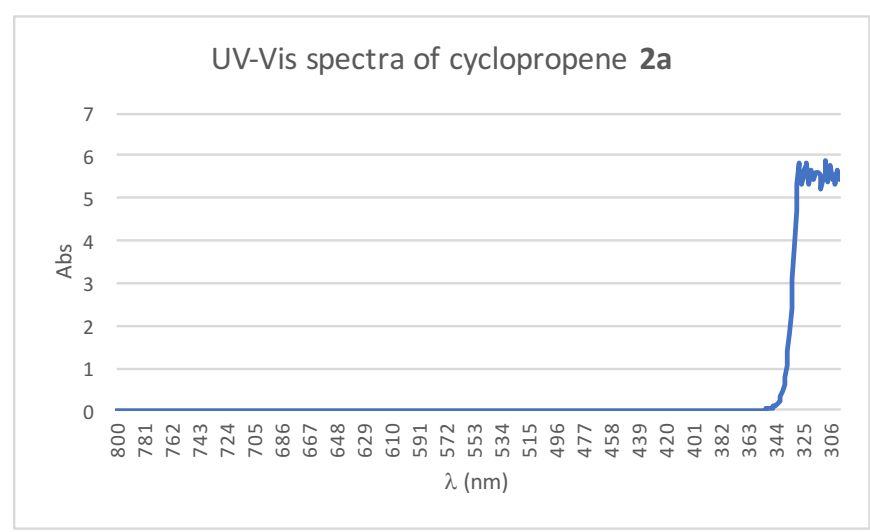

\section{Optimization studies}

4.1 Screening of photochemical cyclopropenation with medium-pressure mercury lamp:

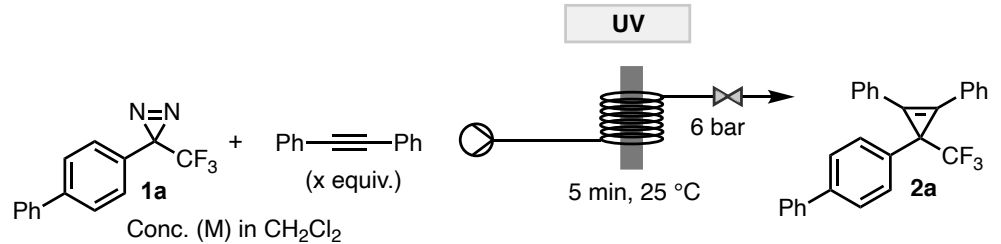

$\begin{array}{ccccc}\text { Entry } & \text { Conc }(\mathbf{M}) & \text { x (equiv) } & \text { Light type } & \text { Yield (\%) } \\ \mathbf{1} & 0.1 & 10 & \text { UV-band pass }(350 \mathrm{~nm}) & 77 \\ \mathbf{2} & 0.1 & 1 & \text { UV-band pass }(350 \mathrm{~nm}) & 35 \\ \mathbf{3} & 0.05 & 10 & \text { UV-band pass }(350 \mathrm{~nm}) & 77 \\ \mathbf{4} & 0.025 & 10 & \text { UV-band pass }(350 \mathrm{~nm}) & 65 \\ \mathbf{5} & 0.1 & 10 & \text { UV-band pass }(320 \mathrm{~nm}) & 76 \\ \mathbf{6} & 0.1 & 10 & \text { UV-band pass }(360 \mathrm{~nm}) & 80 \\ \mathbf{7} & 0.1 & 10 & \text { UV-band pass }(420 \mathrm{~nm}) & 88 \\ \mathbf{8} & 0.1 & 10 & \text { UV-long pass }(280 \mathrm{~nm}) & 75 \\ \mathbf{9} & 0.1 & 10 & \text { UV-long pass }(300 \mathrm{~nm}) & 70\end{array}$


4.1.1 Wavelength filters

- Band-pass wavelength filter:

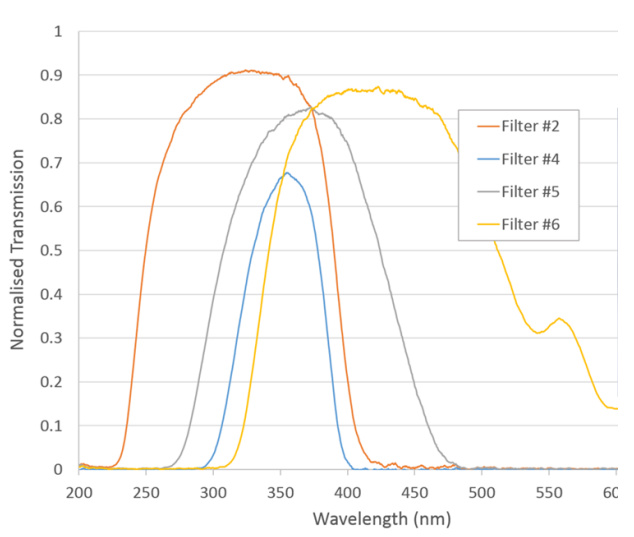

Figure S1a. Image source:

https://www.vapourtec.com/products/flowreactors/photochemistry-uv-150-photochemicalwavelength-filters/
- Long-pass wavelength filter:

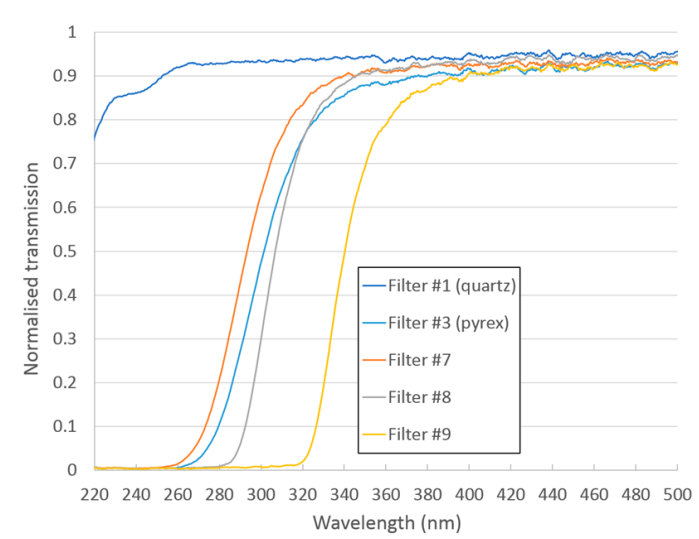

Figure S1b. Image source:

https://www.vapourtec.com/products/flowreactors/photochemistry-uv-150-photochemicalwavelength-filters/

\subsection{Screening of flow conditions for the cyclopropenation of diazirine 1a}

\subsubsection{Screening of photochemical cyclopropenation with light-emitting diodes (LEDs)}

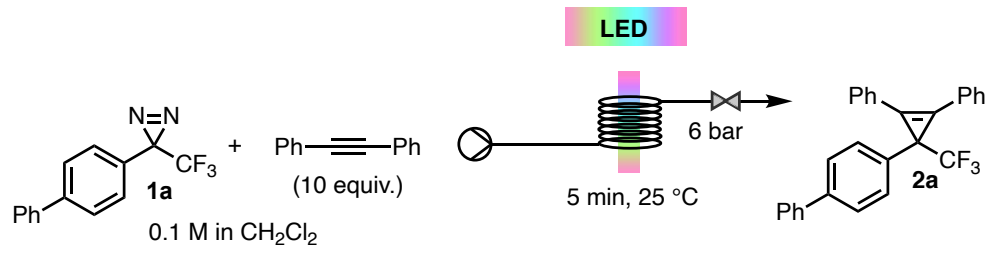

$\begin{array}{lll}\text { Entry } & \text { Light type } & \text { Yield (\%) } \\ 1 & \operatorname{LED}(365 \mathrm{~nm} \text { gen-2) } & 87 \\ 2 & \operatorname{LED}(365 \mathrm{~nm}) & 81 \\ 3 & \operatorname{LED}(380 \mathrm{~nm}) & 81 \\ 4 & \operatorname{LED}(405 \mathrm{~nm}) & 88 \\ 5 & \operatorname{LED}(420 \mathrm{~nm}) & 91 \\ 6 & \operatorname{LED}(450 \mathrm{~nm}) & 10 \\ 7 & \operatorname{LED}(470 \mathrm{~nm}) & 0 \\ 8 & \operatorname{LED}(495 \mathrm{~nm}) & 0 \\ 9 & \operatorname{LED}(525 \mathrm{~nm}) & 0\end{array}$




\subsubsection{Optimization of reaction conditions}

$\begin{array}{llllll}\text { Entry } & \mathbf{R} & \mathbf{x} \text { (equiv) } & \mathrm{Conc}(\mathrm{M}) & \mathrm{T}\left({ }^{\circ} \mathrm{C}\right) & \text { Yield (\%) } \\ 1 & \mathrm{Ph} & 10 & 0.1 & 25 & 91 \\ 2 & \mathrm{Ph} & 5 & 0.1 & 25 & 80 \\ 3 & \mathrm{Ph} & 1.1 & 0.4 & 25 & 43 \\ 4 & \mathrm{Ph} & 10 & 0.2 & 25 & 95 \\ 5 & \mathrm{Ph} & 10 & 0.05 & 25 & 85 \\ 7 & \mathrm{Ph} & 10 & 0.2 & 10 & 93\end{array}$




\section{Experimental procedures for the cyclopropenation reaction of diazirines in continuous flow}

\subsection{Continuous flow setup for cyclopropene synthesis}

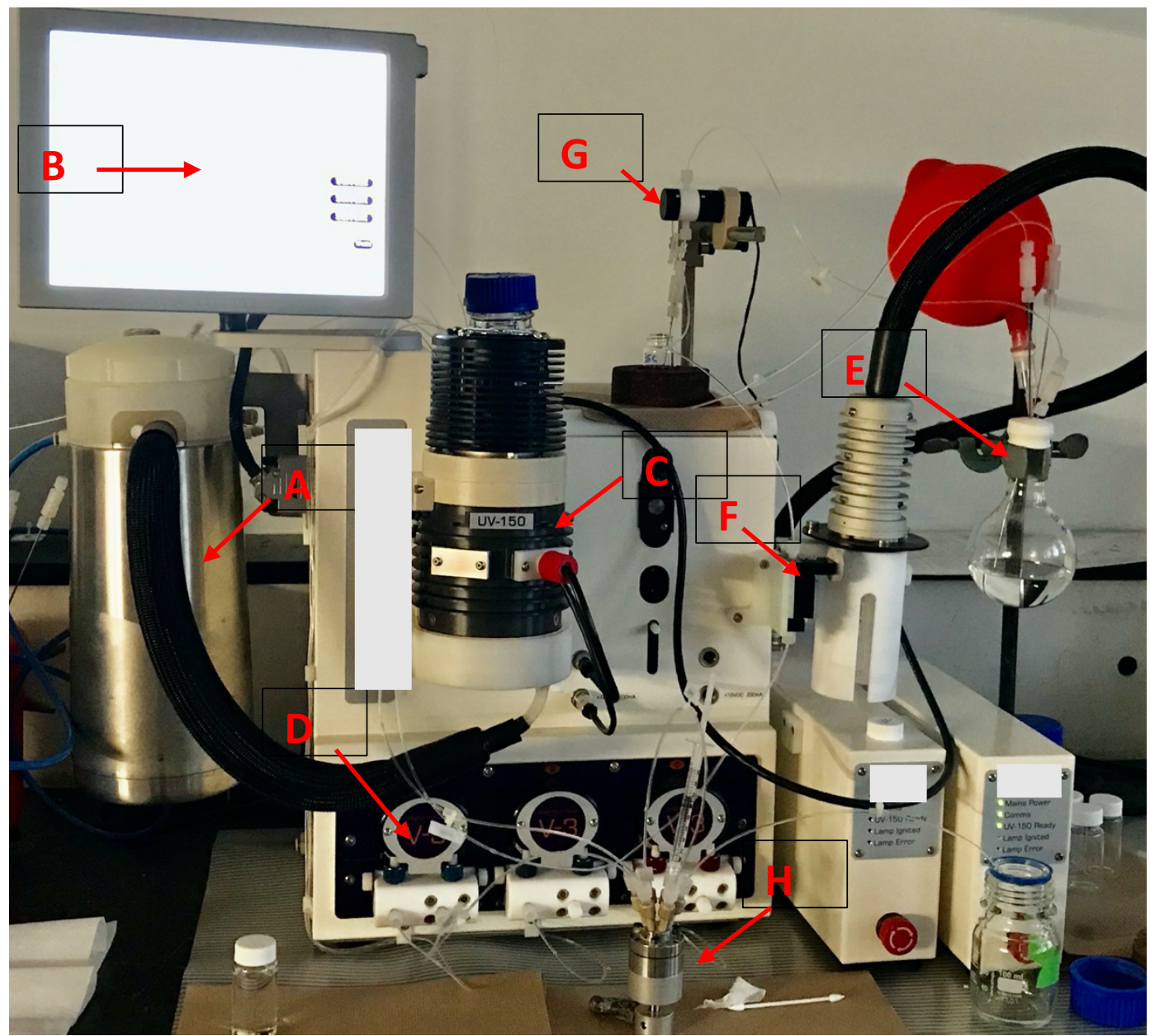

Figure S2. A: Cooling module: filled with dry ice; B: Control monitor; C: UV-150 photochemical reactor equipped with an LED lamp and PFA reactor; D: Peristaltic pump; $\mathbf{E}: \mathrm{CH}_{2} \mathrm{Cl}_{2}$ feeding under argon atmosphere; $\mathbf{F}$ : Back-pressure regulator; G: Collection unit and post-reaction stream collection vial; H: Injection loop (0.25 mL). 


\subsection{General procedure for the photochemical flow reactions of diazirines in continuous flow}

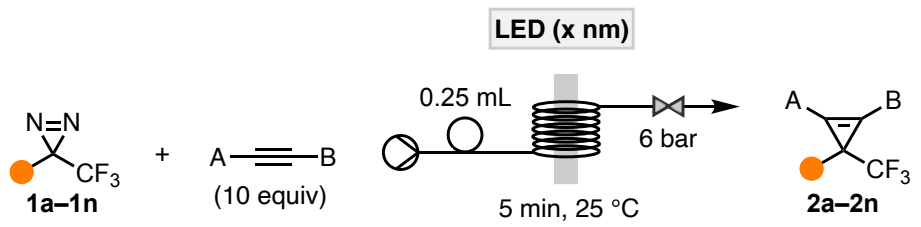

$0.4 \mathrm{M}$ in $\mathrm{CH}_{2} \mathrm{Cl}_{2}$

Flow reactions were set up on a Vapourtec ${ }^{\circledR}$ E-series Easy MedChem ${ }^{\mathrm{TM}}$ flow system. All necessary reactors and accessories were assembled as shown above. Before starting reactions, all reactor tubing were primed with anhydrous $\mathrm{CH}_{2} \mathrm{Cl}_{2}$. Back pressure was set to 6 bar at $2 \mathrm{~mL} / \mathrm{min}$. A solution of the diazirine 1 and alkyne (10 equiv) in $\mathrm{CH}_{2} \mathrm{Cl}_{2}(0.4$ M) was prepared and loaded into the injection loop $(0.25 \mathrm{~mL}$, reaction scale $=0.1 \mathrm{mmol})$. The flow rate was set to 2 $\mathrm{mL} / \mathrm{min}$ for a $10 \mathrm{~mL}$ reactor volume (residence time $=5 \mathrm{~min}$ ). The flow reactions were run under LED irradiation at 25 ${ }^{\circ} \mathrm{C}$. The post-reaction stream containing the desired cyclopropene product (pre- and post-slug volume $=1 \mathrm{~mL}$ ) was collected in a $30 \mathrm{~mL}$ vial. The solvent was removed under reduced pressure and the crude mixture was purified by flash column chromatography (Biotage, SNAP ULTRA, 100:0-99:1 hexanes/EtOAc) to give the desired cyclopropene 2.

\subsubsection{Characterization of 2,2,2-trifluoromethyl cyclopropenes 2a-am}

\section{4-(2,3-Diphenyl-1-(trifluoromethyl)cycloprop-2-en-1-yl)-1,1'-biphenyl 2a}

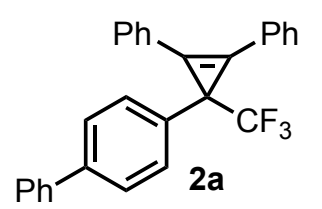

(Purple LED lamp, $420 \mathrm{~nm}$ ). Purified by flash column chromatography (100:0-99:1 hexanes/EtOAc). Isolated as an off-white solid (38 mg, 93\%). ${ }^{*} \mathrm{mp}: 118-120^{\circ} \mathrm{C} .{ }^{1} \mathrm{H}$ NMR $(500$ $\left.\mathrm{MHz}, \mathrm{CDCl}_{3}\right) \delta(\mathrm{ppm}): 7.83-7.77(\mathrm{~m}, 4 \mathrm{H}), 7.61-7.52(\mathrm{~m}, 2 \mathrm{H}), 7.55-7.49(\mathrm{~m}, 8 \mathrm{H}), 7.49-7.39(\mathrm{~m}$, 4H), 7.39-7.29 (m, 1H). ${ }^{13} \mathrm{C}\left\{{ }^{1} \mathrm{H}\right\}$ NMR (126 MHz, $\left.\mathrm{CDCl}_{3}\right) \delta(\mathrm{ppm}): 140.8,140.1,136.5,131.7,130.2,130.0,129.3$, 128.9, 128.1, 127.4, 127.3, 127.1, 126.3, 126.1 (q, $J=279.3 \mathrm{~Hz}), 109.9,33.7$ (q, J = 34.9 Hz). ${ }^{9} \mathrm{~F}$ NMR (470 MHz, $\left.\mathrm{CDCl}_{3}\right) \delta$ (ppm): -62.0. IR (cm-1): 3026, 1495, 1447, 1162, 1126, 920, 750, 681. HR-MS (EI) m/z [M + $\left.\mathrm{NH}_{4}\right]^{+}$calcd. for $\mathrm{C}_{28} \mathrm{H}_{23} \mathrm{~F}_{3} \mathrm{~N} 430.1777$ found 430.1769 .

* This reaction was also run at $10 \mathrm{mmol}$ scale $(3 \mathrm{~g}, 73 \%)$ for a residence time of $50 \mathrm{~min}$.

\section{4-(2-(4-Nitrophenyl)-3-phenyl-1-(trifluoromethyl)cycloprop-2-en-1-yl)-1,1'-biphenyl 2ab}

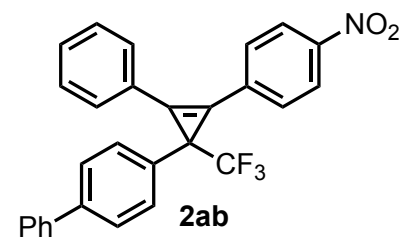

(Purple LED lamp, $420 \mathrm{~nm}$ ). Purified by flash column chromatography (100:0-99:1 hexanes/EtOAc). Isolated as an off-white solid (39 mg, 85\%). $\mathrm{mp}: 110$ $112^{\circ} \mathrm{C} .{ }^{1} \mathrm{H} \mathrm{NMR}\left(500 \mathrm{MHz}, \mathrm{CDCl}_{3}\right) \delta(\mathrm{ppm}): 8.40-8.33(\mathrm{~m}, 2 \mathrm{H}), 7.97-7.88(\mathrm{~m}, 2 \mathrm{H}), 7.83$ (dd, $J=8.1,1.5 \mathrm{~Hz}, 2 \mathrm{H}), 7.56-7.52(\mathrm{~m}, 8 \mathrm{H}), 7.45-7.40(\mathrm{~m}, 3 \mathrm{H}), 7.36-7.31(\mathrm{~m}, 2 \mathrm{H})$. ${ }^{13} \mathrm{C}\left\{{ }^{1} \mathrm{H}\right\} \operatorname{NMR}\left(126 \mathrm{MHz}, \mathrm{CDCl}_{3}\right) \delta(\mathrm{ppm}): 148.0,140.6,140.5,135.4,132.6,131.3,130.8,130.5,129.6,129.0,127.9$, 127.6, 127.5, 127.1, 125.3, 124.7, 115.1, 107.9 ( $\mathrm{CF}_{3}$ signal was not observed). ${ }^{19} \mathrm{~F} \mathrm{NMR}\left(470 \mathrm{MHz}, \mathrm{CDCl}_{3}\right) \delta(\mathrm{ppm}):-$ 
62.2. IR (cm-1): $3103,2214,1591,1508,1344,1103,854,748,686$. HR-MS (EI) m/z [M + H] calcd. for $\mathrm{C}_{28} \mathrm{H}_{19} \mathrm{NO}_{2} \mathrm{~F}_{3}$ 458.1368 found 458.1330 .

\section{1-(4-(3-([1,1'-Biphenyl]-4-yl)-2-phenyl-3-(trifluoromethyl)cycloprop-1-en-1-yl)phenyl)ethan-1-one 2ac}

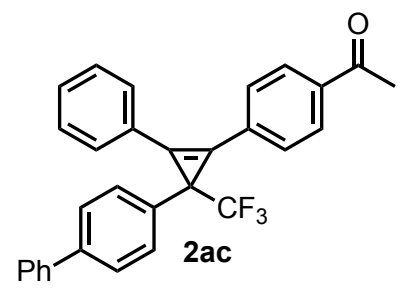

(Purple LED lamp, $420 \mathrm{~nm}$ ). Purified by flash column chromatography (100:0-99:1 hexanes/EtOAc). Isolated as an off-white solid (26 mg, 57\%). mp: 78$80^{\circ} \mathrm{C} .{ }^{1} \mathrm{H} \mathrm{NMR}\left(500 \mathrm{MHz}, \mathrm{CDCl}_{3}\right) \delta(\mathrm{ppm}): 7.98-7.93(\mathrm{~m}, 4 \mathrm{H}), 7.66-7.59$ (m, 4H), 7.58$7.51(\mathrm{~m}, 5 \mathrm{H}), 7.45-7.30(\mathrm{~m}, 5 \mathrm{H}), 2.63(\mathrm{~s}, 3 \mathrm{H}) .{ }^{13} \mathrm{C}\left\{{ }^{1} \mathrm{H}\right\} \mathrm{NMR}\left(126 \mathrm{MHz}, \mathrm{CDCl}_{3}\right) \delta(\mathrm{ppm})$ : $197.3,136.2,131.8,131.7,130.6,130.4,130.0,129.4,129.1,128.9,128.8,128.5$, 128.3, 128.2, 127.3, 127.0, 125.6, 122.7, 92.8, 88.7, 26.6 ( $\mathrm{CF}_{3}$ signal was not observed). ${ }^{19} \mathrm{~F} \mathrm{NMR}\left(470 \mathrm{MHz}, \mathrm{CDCl}_{3}\right)$ $\delta$ (ppm): -62.1. IR (cm-1): 2999, 2220, 1678, 1605, 1485, 1404, 1261, 959, 833, 756, 690. HR-MS (El) m/z [M + H] calcd. for $\mathrm{C}_{30} \mathrm{H}_{22} \mathrm{OF}_{3} 455.1617$ found 455.1628 .

\section{4-(2-Phenyl-1-(trifluoromethyl)-3-(4-(trifluoromethyl)phenyl)cycloprop-2-en-1-yl)-1,1'-biphenyl 2ad}

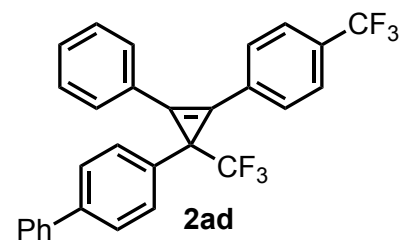

(Purple LED lamp, $420 \mathrm{~nm}$ ). Purified by flash column chromatography (100:0-99:1 hexanes/EtOAc). Isolated as an off-white solid (40 mg, 83\%). mp: 118$120{ }^{\circ} \mathrm{C} .{ }^{1} \mathrm{H}$ NMR ( $\left.500 \mathrm{MHz}, \mathrm{CDCl}_{3}\right) \delta(\mathrm{ppm}): 7.90(\mathrm{~d}, J=8.8 \mathrm{~Hz}, 2 \mathrm{H}), 7.84-7.80$ (m, 2H), $7.77(\mathrm{~d}, J=7.9 \mathrm{~Hz}, 2 \mathrm{H}), 7.59-7.46(\mathrm{~m}, 10 \mathrm{H}), 7.43(\mathrm{t}, J=8.0 \mathrm{~Hz}, 2 \mathrm{H}), 7.36-7.31$ (m, 1H). ${ }^{13} \mathrm{C}\{1 \mathrm{H}\} \mathrm{NMR}\left(126 \mathrm{MHz}, \mathrm{CDCl}_{3}\right) \delta(\mathrm{ppm}): 140.5,140.2,135.7,131.3$ (q, J = $\left.32.8 \mathrm{~Hz}\right), 130.6,130.4,130.0$, 129.6, 129.3, 128.8, 127.8 (q, J = 1.4 Hz), 127.4, 127.3, 127.1, 127.0, 126.2 (q, J = 3.8 Hz), 125.5, 124.9, 122.7, 112.7, 108.4, 68.2. ( $\mathrm{CF}_{3}$ signal was not observed). ${ }^{19} \mathrm{~F} \mathrm{NMR}\left(470 \mathrm{MHz}, \mathrm{CDCl}_{3}\right) \delta(\mathrm{ppm}):-62.1,-62.8 . \mathrm{IR}\left(\mathrm{cm}^{-1}\right): 2926,1616$, 1325, 1159, 1121, 1108, 1065, 837, 739, 687. HR-MS (EI) m/z [M + H] calcd. for $\mathrm{C}_{29} \mathrm{H}_{18} \mathrm{~F}_{6} 481.1391$ found 481.1361.

\section{4-(2-(4-Fluorophenyl)-3-phenyl-1-(trifluoromethyl)cycloprop-2-en-1-yl)-1,1'-biphenyl 2ae}

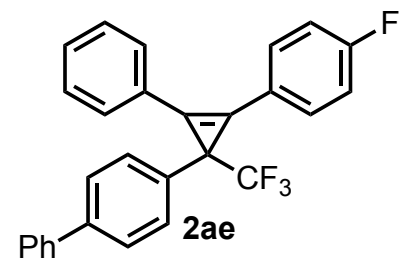

(Purple LED lamp, $420 \mathrm{~nm}$ ). Purified by flash column chromatography (100:0-99:1 hexanes/EtOAc). Isolated as a semi-solid (26 mg, $62 \%)$. ${ }^{1} \mathrm{H} \mathrm{NMR}\left(500 \mathrm{MHz}, \mathrm{CDCl}_{3}\right) \delta$ (ppm): 7.62-7.56 (m, 2H), 7.56-7.52 (m, 2H), 7.49-7.40 (m, 2H), 7.44-7.31 (m, 3H), $2.50(\mathrm{~d}, J=8.5 \mathrm{~Hz}, 4 \mathrm{H}), 1.76-1.61(\mathrm{~m}, 4 \mathrm{H}), 1.02(\mathrm{t}, J=7.5 \mathrm{~Hz}, 6 \mathrm{H})$. ${ }^{13} \mathrm{C}\left\{{ }^{1} \mathrm{H}\right\} \mathrm{NMR}\left(126 \mathrm{MHz}, \mathrm{CDCl}_{3}\right) \delta(\mathrm{ppm}): 163.6(\mathrm{~d}, J=252.1 \mathrm{~Hz}), 140.5(\mathrm{~d}, J=59.3 \mathrm{~Hz}), 136.3,132.3,132.1(\mathrm{~d}, J=$ 8.6 Hz), 130.1, 130.1, 129.4, 128.8, 128.0, 127.9, 127.5, 127.5 (q, J = 266.7 Hz), 127.4, 127.1, 122.61 (d, J = 3.5 $\mathrm{Hz}), 116.7(\mathrm{~d}, J=22.3 \mathrm{~Hz}), 109.4,108.9,33.7(\mathrm{q}, J=35.1 \mathrm{~Hz}) .{ }^{19} \mathrm{~F} \mathrm{NMR}\left(470 \mathrm{MHz}, \mathrm{CDCl}_{3}\right) \delta(\mathrm{ppm}):-62.0,-109.0(\mathrm{p}$, $J=7.4 \mathrm{~Hz}$ ). IR (cm-1): 2922, 1601, 1506, 1484, 1447, 1294, 1234, 1163, 1125, 924, 835, 760, 687. HR-MS (EI) m/z [M $+\mathrm{H}^{+}$calcd. for $\mathrm{C}_{28} \mathrm{H}_{19} \mathrm{~F}_{4} 431.1423$ found 431.1447 . 


\section{4-(2,3-Dipropyl-1-(trifluoromethyl)cycloprop-2-en-1-yl)-1,1'-biphenyl 2af}

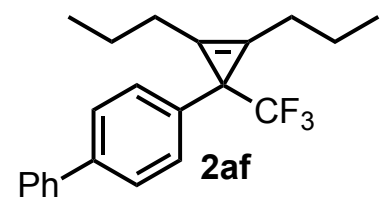

(Purple LED lamp, $420 \mathrm{~nm}$ ). Purified by flash column chromatography (100:0-99:1 hexanes/EtOAc). Isolated as a semi-solid (33 mg, $97 \%)$. ${ }^{1} \mathrm{H} \mathrm{NMR}\left(500 \mathrm{MHz}, \mathrm{CDCl}_{3}\right) \delta$ (ppm): 7.82-7.73 (m, 4H), 7.60-7.49 (m, 8H), 7.46-7.39 (m, 4H), 7.36-7.32 (m, 1H), 7.24-7.19 (m, 2H). ${ }^{13}{ }^{2}\left\{{ }^{1} \mathrm{H}\right\} \mathrm{NMR}\left(126 \mathrm{MHz}, \mathrm{CDCl}_{3}\right) \delta(\mathrm{ppm}): 140.8,139.2,138.8,128.7$, 127.8, 127.4 (q, J = 277.6 Hz), 127.2, 127.0, 127.0, 109.6, 32.8 (q, J = 34.4 Hz), 25.8, 20.6, 14.0. ${ }^{19} \mathrm{~F} \mathrm{NMR}$ (470 MHz, $\left.\mathrm{CDCl}_{3}\right) \delta(\mathrm{ppm}):-62.1 . \mathrm{IR}\left(\mathrm{cm}^{-1}\right): 2962,1489,1300,1151,1115,920,696$. HR-MS (EI) $\mathrm{m} / \mathrm{z}[\mathrm{M}+\mathrm{H}]^{+}$calcd. for $\mathrm{C}_{22} \mathrm{H}_{24} \mathrm{~F}_{3}$ 345.1830 found 345.1836 .

\section{4-(2-Phenyl-1-(trifluoromethyl)cycloprop-2-en-1-yl)-1,1'-biphenyl 2ag}

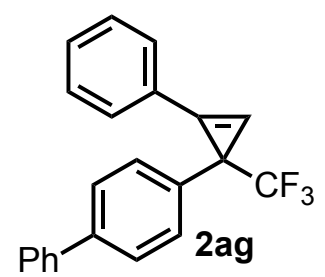

(Purple LED lamp, $420 \mathrm{~nm}$ ). Purified by flash column chromatography (100:0-99:1 hexanes/EtOAc). Isolated as a white solid $(20 \mathrm{mg}, 60 \%)$. mp: $120-122{ }^{\circ} \mathrm{C}$. ${ }^{1} \mathrm{H}$ NMR $\left(500 \mathrm{MHz}, \mathrm{CDCl}_{3}\right) \delta$ (ppm): 7.77-7.64 (m, 2H), 7.60-7.54 (m, 4H), 7.54-7.42 (m, $8 \mathrm{H}), 7.40-7.32(\mathrm{~m}, 1 \mathrm{H}), 7.23-7.19(\mathrm{~m}, 1 \mathrm{H}) .{ }^{13} \mathrm{C}\left\{{ }^{\prime} \mathrm{H}\right\} \mathrm{NMR}\left(126 \mathrm{MHz}, \mathrm{CDCl}_{3}\right) \delta(\mathrm{ppm}): 140.8$, 140.2, 137.3, 130.6, 130.2, 129.2, 128.9, 128.2, 127.4, 127.3, 127.2, 125.7 (q, J = 277.8 Hz),

125.1, 117.4, 98.9 (q, J = 3.1 Hz), 32.0 (q, J = 36.4 Hz). ${ }^{19 F} \operatorname{NMR}\left(470 \mathrm{MHz}, \mathrm{CDCl}_{3}\right) \delta(\mathrm{ppm}):-63.9 . \mathrm{IR}\left(\mathrm{cm}^{-1}\right): 2962$, 1489, 1300, 1151, 1115, 920, 696. HR-MS (EI) $\mathrm{m} / \mathrm{z}[\mathrm{M}+\mathrm{H}]^{+}$calcd. for $\mathrm{C}_{22} \mathrm{H}_{16} \mathrm{~F}_{3} 337.1198$ found 337.1190 .

\section{4-(2-(p-Tolyl)-1-(trifluoromethyl)cycloprop-2-en-1-yl)-1,1'-biphenyl 2ah}

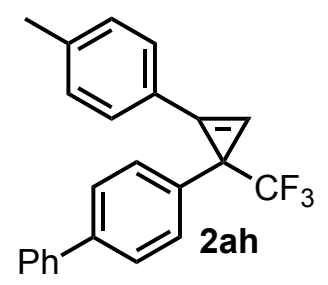

(Purple LED lamp, $420 \mathrm{~nm}$ ). Purified by flash column chromatography (100:0-99:1 hexanes/EtOAc). Isolated as a white solid $(21 \mathrm{mg}, 61 \%)$. mp: $112-114{ }^{\circ} \mathrm{C}$. ${ }^{1} \mathrm{H} \mathrm{NMR}\left(500 \mathrm{MHz}, \mathrm{CDCl}_{3}\right) \delta(\mathrm{ppm}): 7.62-7.47(\mathrm{~m}, 8 \mathrm{H}), 7.49-7.40(\mathrm{~m}, 2 \mathrm{H}), 7.37-7.32(\mathrm{~m}, 1 \mathrm{H})$, 7.30-7.27 (m, 1H), $7.12(\mathrm{q}, J=1.5 \mathrm{~Hz}, 1 \mathrm{H}), 2.42(\mathrm{~s}, 3 \mathrm{H}) .{ }^{13} \mathrm{C}\left\{{ }^{1} \mathrm{H}\right\} \mathrm{NMR}\left(126 \mathrm{MHz}, \mathrm{CDCl}_{3}\right)$ $\delta$ (ppm): 140.9, 140.7, 140.0, 137.4, 130.0, 129.8, 128.8, 128.0 (q, J = 1.5 Hz), 127.3, 127.1, 127.1, $125.7(q, J=277.4 \mathrm{~Hz}), 122.2,117.0(q, J=2.2 \mathrm{~Hz}), 97.6(q, J=2.8 \mathrm{~Hz}), 31.7(q, J=34.9 \mathrm{~Hz}), 21.6 .{ }^{19} \mathrm{~F} \mathrm{NMR}$ (470 MHz, CDCl 3 ) $\delta(\mathrm{ppm}):-63.9 . \mathrm{IR}\left(\mathrm{cm}^{-1}\right): 3130,1487,1296,1153,1111,922,820,696 . \mathrm{HR}-\mathrm{MS}$ (EI) m/z [M + H] ${ }^{+}$ calcd. for $\mathrm{C}_{23} \mathrm{H}_{18} \mathrm{~F}_{3} 351.1355$ found 351.1326 .

\section{4-(2-(4-Ethylphenyl)-1-(trifluoromethyl)cycloprop-2-en-1-yl)-1,1'-biphenyl 2ai}<smiles>CCc1ccc(C2=CC2(c2ccc(-c3ccccc3)cc2)C(F)(F)F)cc1</smiles>

(Purple LED lamp, $420 \mathrm{~nm}$ ). Purified by flash column chromatography (100:0-99:1 hexanes/EtOAc). Isolated as a white solid (22 mg, 61\%). mp: 88-90 ${ }^{\circ} \mathrm{C}$. ${ }^{1} \mathrm{H} \mathrm{NMR}\left(400 \mathrm{MHz}, \mathrm{CDCl}_{3}\right) \delta(\mathrm{ppm}): 7.64-7.46(\mathrm{~m}, 8 \mathrm{H}), 7.43(\mathrm{t}, J=7.7 \mathrm{~Hz}, 2 \mathrm{H}), 7.38-7.24$ $(\mathrm{m}, 3 \mathrm{H}), 7.11(\mathrm{~m}, 1 \mathrm{H}), 2.70(\mathrm{q}, J=7.5 \mathrm{~Hz}, 2 \mathrm{H}), 1.26(\mathrm{t}, J=7.6 \mathrm{~Hz}, 3 \mathrm{H}) .{ }^{13} \mathrm{C}\left\{{ }^{1} \mathrm{H}\right\} \mathrm{NMR}(126$ $\left.\mathrm{MHz}, \mathrm{CDCl}_{3}\right) \delta(\mathrm{ppm}): 147.3,140.8,140.2,137.5,130.3,128.9,128.7,128.2,127.4,127.3$, 
127.2, $125.8(q, J=279.1 \mathrm{~Hz}), 122.5,117.2(q, J=2.3 \mathrm{~Hz}), 97.7(q, J=3.0 \mathrm{~Hz}), 32.0$ (q, $J=35.4 \mathrm{~Hz}), 29.1,15.5 .{ }^{19} \mathrm{~F}$ $\operatorname{NMR}\left(376 \mathrm{MHz}, \mathrm{CDCl}_{3}\right) \delta$ (ppm): -63.9. IR (cm-1): 3130, 1489, 1298, 1154, 1110, 922, 837, 768, 694. HR-MS (EI) m/z $[\mathrm{M}+\mathrm{H}]^{+}$calcd. for $\mathrm{C}_{24} \mathrm{H}_{20} \mathrm{~F}_{3} 365.1512$ found 365.1568 .

\section{4-(2-(4-Bromophenyl)-1-(trifluoromethyl)cycloprop-2-en-1-yl)-1,1'-biphenyl 2aj}

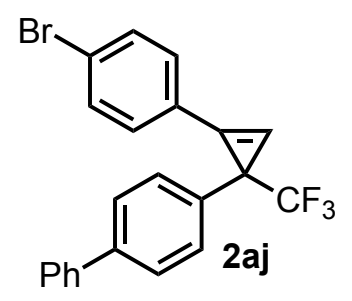

(Purple LED lamp, $420 \mathrm{~nm}$ ). Purified by flash column chromatography (100:0-99:1 hexanes/EtOAc). Isolated as a white solid (26 mg, 62\%). mp: $98-100{ }^{\circ} \mathrm{C}$. ${ }^{1} \mathrm{H}$ NMR (500 MHz, $\left.\mathrm{CDCl}_{3}\right) \delta(\mathrm{ppm}): 7.64-7.60(\mathrm{~m}, 2 \mathrm{H}), 7.59-7.52(\mathrm{~m}, 6 \mathrm{H}), 7.51-7.42(\mathrm{~m}$, $4 \mathrm{H}), 7.42-7.31(\mathrm{~m}, 1 \mathrm{H}), 7.25(\mathrm{q}, J=1.5 \mathrm{~Hz}, 1 \mathrm{H}) .{ }^{13} \mathrm{C}\left\{{ }^{1} \mathrm{H}\right\} \mathrm{NMR}\left(126 \mathrm{MHz}, \mathrm{CDCl}_{3}\right) \delta(\mathrm{ppm})$ : $140.7,140.4,136.9,132.5,131.5,128.9,128.1,127.5,127.4,127.2,126.6$ (q, $J=277.7$ $\mathrm{Hz}), 125.1,124.1,116.6,99.9$ (q, J = 3.2 Hz), 32.2 (q, J = 35.4 Hz). $\left.{ }^{19} \mathrm{~F} \mathrm{NMR} \mathrm{(470} \mathrm{MHz,} \mathrm{CDCl}_{3}\right) \delta$ (ppm): -64.0. IR $\left(\mathrm{cm}^{-1}\right): 3128,1479,1294,1154,1112,922,829,694$. HR-MS (EI) $\mathrm{m} / \mathrm{z}[\mathrm{M}+\mathrm{H}]^{+}$calcd. for $\mathrm{C}_{22} \mathrm{H}_{15} \mathrm{~F}_{3} \mathrm{Br} 415.0309$ found 415.0347.

\section{(3-([1,1'-Biphenyl]-4-yl)-3-(trifluoromethyl)cycloprop-1-en-1-yl)trimethylsilane 2ak}

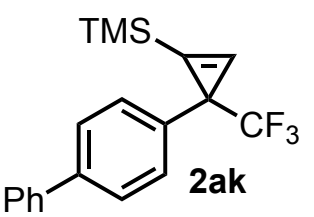

(Purple LED lamp, $420 \mathrm{~nm}$ ). Purified by flash column chromatography (100:0-99:1 hexanes/EtOAc). Isolated as a white solid (26 mg, 78\%). mp: $48-50{ }^{\circ} \mathrm{C}$. ${ }^{1} \mathrm{H} \mathrm{NMR}\left(500 \mathrm{MHz}, \mathrm{CDCl}_{3}\right) \delta$ (ppm): $7.60(\mathrm{~d}, J=6.0 \mathrm{~Hz}, 2 \mathrm{H}), 7.57-7.51(\mathrm{~m}, 3 \mathrm{H}), 7.45$ (t, $J=$ $8.0 \mathrm{~Hz}, 2 \mathrm{H}), 7.38-7.34(\mathrm{~m}, 3 \mathrm{H}), 0.26-0.24(\mathrm{~m}, 9 \mathrm{H}) .{ }^{13} \mathrm{C}\left\{{ }^{1} \mathrm{H}\right\} \mathrm{NMR}\left(126 \mathrm{MHz}, \mathrm{CDCl}_{3}\right) \delta(\mathrm{ppm})$ : 140.8, 139.6, 139.3, 128.9, 127.9, 127.7, 127.4, 127.2, 126.2 (q, J = 276.8 Hz), 127.0, 116.5 (q, J = 2.2 Hz), 29.9 (q, J $=35.5 \mathrm{~Hz}),-1.40 .{ }^{19} \mathrm{~F} \mathrm{NMR}\left(470 \mathrm{MHz}, \mathrm{CDCl}_{3}\right) \delta(\mathrm{ppm}):-63.5 . \mathrm{IR}\left(\mathrm{cm}^{-1}\right): 2965,1703,1489,1304,1153,1113,924$, 839, 727, 692. HR-MS (El) m/z [M + NH $\left.]_{4}\right]^{+}$calcd. for $\mathrm{C}_{19} \mathrm{H}_{23} \mathrm{~F}_{3} \mathrm{NSi} 350.1546$ found 350.1575 .

\section{4-(2-(3-Ethynylphenyl)-1-(trifluoromethyl)cycloprop-2-en-1-yl)-1,1'-biphenyl 2al}

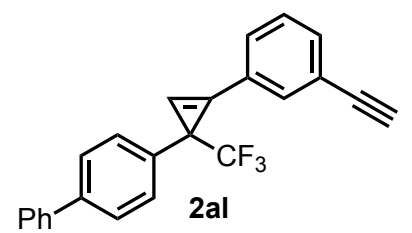

(Purple LED lamp, $420 \mathrm{~nm}$ ). Purified by flash column chromatography (100:0-99:1 hexanes/EtOAc). Isolated as a white solid (19 mg, 53\%). mp: $82-84{ }^{\circ} \mathrm{C}$. ${ }^{1} \mathrm{H} \mathrm{NMR}\left(500 \mathrm{MHz}, \mathrm{CDCl}_{3}\right) \delta$ (ppm): 7.79 (br s, 1H), 7.66-7.63 (m, 1H), 7.57-7.54 (m, 5H), 7.48-7.47 (m, 2H), 7.44-7.41 (m, 3H), 7.36-7.32 (m, 1H), 7.25 (br s, 1H), 3.14 (s, 1H). ${ }^{13} \mathrm{C}\left\{{ }^{1} \mathrm{H}\right\} \operatorname{NMR}\left(126 \mathrm{MHz}, \mathrm{CDCl}_{3}\right) \delta(\mathrm{ppm}): 140.7,140.4,137.0,134.1,133.5,130.3,129.3,128.9,128.1,127.5$, $127.4,127.2,126.2$ (q, $J=277.8 \mathrm{~Hz}), 125.5,123.4,116.8,100.3$ (q, $J=2.9 \mathrm{~Hz}), 82.6,78.6,32.3$ (q, $J=35.7 \mathrm{~Hz}) .{ }^{19} \mathrm{~F}$ $\operatorname{NMR}\left(470 \mathrm{MHz}, \mathrm{CDCl}_{3}\right) \delta$ (ppm): -64.0. IR (cm-1): 3276, 3128, 2924, 1717, 1487, 1298, 1147, 1121, 921, 723, 692. HR-MS (EI) m/z [M + H] $]^{+}$calcd. for $\mathrm{C}_{24} \mathrm{H}_{16} \mathrm{~F}_{3} 361.1199$ found 361.1061 . 


\section{4-(4-(3-Ethynylphenyl)-1,1,1-trifluorobut-3-yn-2-yl)-1,1'-biphenyl 2al'}

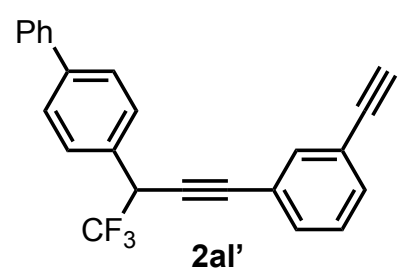

(Purple LED lamp, $420 \mathrm{~nm}$ ). Purified by flash column chromatography (100:0-99:1 hexanes/EtOAc). Isolated as a white solid (7 mg, 19\%). mp: $50-52{ }^{\circ} \mathrm{C}$. ${ }^{1} \mathrm{H}$ NMR (500 MHz, $\left.\mathrm{CDCl}_{3}\right) \delta$ (ppm): 7.74-7.69 (m, 3H), 7.62-7.58 (m, 2H), 7.57-7.56 (m, 2H), 7.47-7.44 (m, 3H), 7.39-7.36 (m, 1H), 6.49 (d, J=2.2 Hz, 1H), 4.31 (q, J = 9.9 $\mathrm{Hz}, 1 \mathrm{H}), 3.13$ (s, 1H). ${ }^{13} \mathrm{C}\left\{{ }^{1} \mathrm{H}\right\} \mathrm{NMR}\left(126 \mathrm{MHz}, \mathrm{CDCl}_{3}\right) \delta(\mathrm{ppm}): 148.6,144.6,142.2$, 141.2, 134.9, 132.3, 131.5, 129.4, 129.0, 128.3, 127.6, 126.2 (q, $J=2.7 \mathrm{~Hz}$ ), 126.1 (q, $J=279.9 \mathrm{~Hz}), 125.9,125.2$, 122.8, 120.1, 83.3, 78.0, 52.6 (q, $J=29.7 \mathrm{~Hz}) .{ }^{19} \mathrm{~F} \mathrm{NMR}\left(470 \mathrm{MHz}, \mathrm{CDCl}_{3}\right) \delta(\mathrm{ppm}):-67.2$ (d, $\left.J=8.7 \mathrm{~Hz}\right)$. IR (cm-1): 3294, 2924, 1601, 1490, 1323, 1256, 1149, 1103, 760, 692, 624. HR-MS (EI) m/z [M + NH $\left.]^{+}\right]^{\text {calcd. for }}$ $\mathrm{C}_{24} \mathrm{H}_{19} \mathrm{~F}_{3} \mathrm{~N} 378.1464$ found 378.1433 .

Despite multiple attempts, compound $\mathbf{2 a m}$ was not isolated and could only be identified in the crude reaction mixture. 2-(2,3-Diphenyl-1-(trifluoromethyl)cycloprop-2-en-1-yl)naphthalene $2 b$

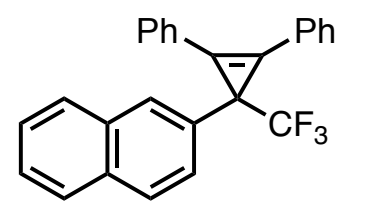

2b

(Purple LED lamp, $420 \mathrm{~nm}$ ). Purified by flash column chromatography (100:0-99:1 hexanes/EtOAc). Isolated as a white solid (32 mg, 78\%). mp: $82-84{ }^{\circ} \mathrm{C}$. ${ }^{1} \mathrm{H} \mathrm{NMR}\left(500 \mathrm{MHz}, \mathrm{CDCl}_{3}\right) \delta(\mathrm{ppm}): 8.06$ (br s, 1H), 7.83-7.81 (m, 4H), 7.79-7.74 (m, 3H), 7.55-7.49 (m, 5H), 7.46-7.43 (m, 4H). ${ }^{13} \mathrm{C}\left\{{ }^{1} \mathrm{H}\right\} \mathrm{NMR}\left(126 \mathrm{MHz}, \mathrm{CDCl}_{3}\right) \delta(\mathrm{ppm}): 135.0$, $133.4,132.5,131.8,130.2,130.0,129.3,128.2,128.1,127.6,126.6,126.3,126.3,126.0,125.4,127.2$ (q, J = 278.8 $\mathrm{Hz}), 109.9,34.0$ (q, J = 34.9 Hz). ${ }^{19} \mathrm{~F} \mathrm{NMR}\left(470 \mathrm{MHz}, \mathrm{CDCl}_{3}\right) \delta(\mathrm{ppm}):-61.7 . \mathrm{IR}\left(\mathrm{cm}^{-1}\right): 3060,1447,1286,1153,1121$, 752, 685. HR-MS (El) $\mathrm{m} / \mathrm{z}[\mathrm{M}+\mathrm{H}]^{+}$calcd. for $\mathrm{C}_{26} \mathrm{H}_{19} \mathrm{~F}_{3} 387.1355$ found 387.1358.

\section{(3-(4-(benzyloxy)phenyl)-3-(trifluoromethyl)cycloprop-1-ene-1,2-diyl)dibenzene 2c}<smiles>FC(F)(F)C1(c2ccccc2)C(c2ccccc2)=C1c1ccccc1</smiles>

(Purple LED lamp, $420 \mathrm{~nm}$ ). Purified by flash column chromatography (100:0-99:1 hexanes/EtOAc). Isolated as a white solid (26 mg, 79\%). mp: $90-92{ }^{\circ} \mathrm{C}$. ${ }^{1} \mathrm{H}$ NMR (500 MHz, $\left.\mathrm{CDCl}_{3}\right) \delta(\mathrm{ppm}):$ 7.81-7.79 (m, 3H), 7.53-7.50 (m, 3H), 7.47-7.37 (m, $9 \mathrm{H}), 7.34-7.31(\mathrm{~m}, 1 \mathrm{H}), 6.93-6.91(\mathrm{~m}, 2 \mathrm{H}), 5.03(\mathrm{~s}, 2 \mathrm{H}) .{ }^{13} \mathrm{C}\left\{{ }^{1} \mathrm{H}\right\} \mathrm{NMR}\left(126 \mathrm{MHz}, \mathrm{CDCl}_{3}\right)$ $\delta$ (ppm): 158.1, 137.1, 130.1, 130.7, 130.1, 129.9, 129.3, 129.1, 128.7, 128.1, 127.6, 127.2 (q, J = 280.2 Hz), 126.5, 114.9, 110.5, 70.1, 33.2 (q, $J=34.9 \mathrm{~Hz})$. ${ }^{19} \mathrm{~F} \quad \mathrm{NMR}\left(470 \mathrm{MHz}, \mathrm{CDCl}_{3}\right) \delta$ (ppm): -62.4 . IR (cm-1): 3025, 1609, 1512, 1445, 1288, 1242, 1153, 1124, 1009, 835, 746, 683. HR-MS (El) m/z [M + NH4 $]^{+}$calcd. for $\mathrm{C}_{29} \mathrm{H}_{25} \mathrm{ONF}_{3} 460.1883$ found 460.1847 . 


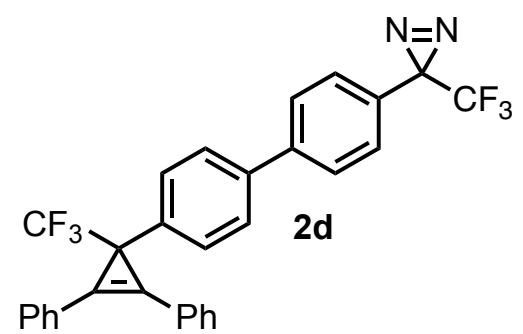

(Purple LED lamp, $420 \mathrm{~nm}$ ). Purified by flash column chromatography (100:0$99: 1$ hexanes/EtOAc). Isolated as a white solid (13 mg, 30\%). mp: $58-$ $60{ }^{\circ} \mathrm{C} .{ }^{1} \mathrm{H}$ NMR $\left(500 \mathrm{MHz}, \mathrm{CDCl}_{3}\right) \delta(\mathrm{ppm}): 7.82-7.76(\mathrm{~m}, 5 \mathrm{H}), 7.57-7.47(\mathrm{~m}$, $8 \mathrm{H}), 7.47-7.41(\mathrm{~m}, 5 \mathrm{H}) .{ }^{13} \mathrm{C}\left\{{ }^{1} \mathrm{H}\right\} \mathrm{NMR}\left(126 \mathrm{MHz}, \mathrm{CDCl}_{3}\right) \delta(\mathrm{ppm}): 139.4,136.4$, $130.0,129.8,129.2,127.9,127.0,126.1,109.8$ (q, J = 2.5 Hz), 33.6 (q, $J=33.8$ $\mathrm{Hz}) .{ }^{19} \mathrm{~F} \mathrm{NMR}\left(470 \mathrm{MHz}, \mathrm{CDCl}_{3}\right) \delta(\mathrm{ppm}):-62.0,-65.1$. IR $\left(\mathrm{cm}^{-1}\right): 2924,1716,1265,1168,1126,1029,754,683$. HRMS (El) $\mathrm{m} / \mathrm{z}[\mathrm{M}+\mathrm{H}]^{+}$calcd. for $\mathrm{C}_{30} \mathrm{H}_{19} \mathrm{~F}_{6} \mathrm{~N}_{2} 521.1452$ found 521.1434 .

\section{4,4'-Bis(2,3-diphenyl-1-(trifluoromethyl)cycloprop-2-en-1-yl)-1,1'-biphenyl 2d'}

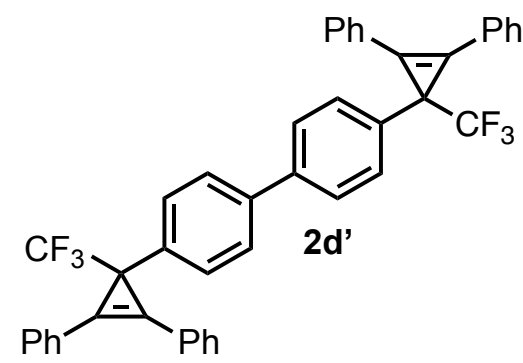

(Purple LED lamp, $420 \mathrm{~nm}$ ). Purified by flash column chromatography (100:099:1 hexanes/EtOAc). Isolated as a white solid (36 mg, 54\%). $\mathrm{mp}$ (decomp.): $219-221^{\circ} \mathrm{C} .{ }^{1} \mathrm{H} \mathrm{NMR}\left(500 \mathrm{MHz}, \mathrm{CDCl}_{3}\right) \delta$ (ppm): $7.82-7.75$ $(\mathrm{m}, 8 \mathrm{H}), 7.57-7.48(\mathrm{~m}, 12 \mathrm{H}), 7.48-7.41(\mathrm{~m}, 8 \mathrm{H}).) .{ }^{13} \mathrm{C}\left\{{ }^{1} \mathrm{H}\right\} \mathrm{NMR}(126 \mathrm{MHz}$, $\left.\mathrm{CDCl}_{3}\right) \delta$ (ppm): 139.5, 136.5, 130.2, 130.0, 129.3, 128.1, 127.2, 126.3, 110.0, 33.6 (q, J = 34.6 Hz). $\left.{ }^{19} \mathrm{~F} \mathrm{NMR} \mathrm{(470} \mathrm{MHz,} \mathrm{CDCl}_{3}\right) \delta(\mathrm{ppm}):-62.01 . \mathrm{IR}\left(\mathrm{cm}^{-1}\right)$ : $3078,1497,1448,1295,1145,1119,921,754,685$. HR-MS (El) m/z [M + NH $]_{4}^{+}$calcd. for $\mathrm{C}_{44} \mathrm{H}_{32} \mathrm{NF}_{6} 688.2433$ found 688.2444.

\section{(3-(p-Tolyl)-3-(trifluoromethyl)cycloprop-1-ene-1,2-diyl)dibenzene $2 \mathrm{e}$}

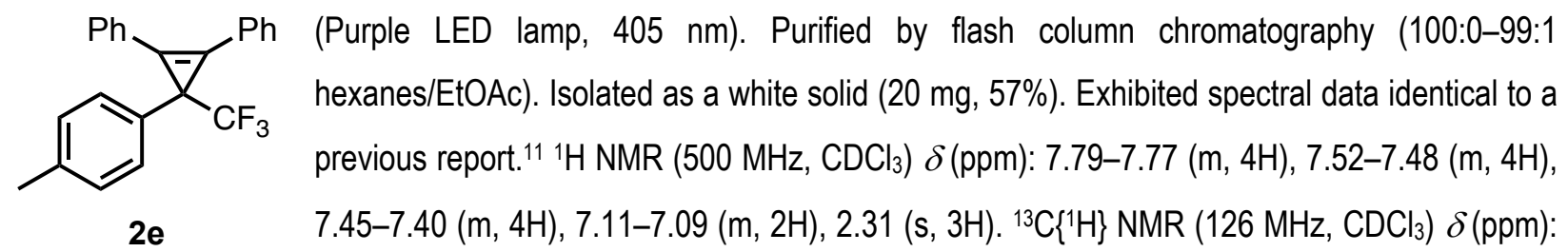
$137.9,134.5,131.8,130.5,130.1,129.9,129.3,129.2,127.6,127.3$ (q, J = 280.2 Hz), 110.2 (q, J = 2.4 Hz), 33.6 (q, J $=34.9 \mathrm{~Hz}), 21.2 .{ }^{19} \mathrm{~F} \mathrm{NMR}\left(470 \mathrm{MHz}, \mathrm{CDCl}_{3}\right) \delta(\mathrm{ppm}):-62.2$.

\section{(3-(4-Tert-butylphenyl)-3-(trifluoromethyl)cycloprop-1-ene-1,2-diyl)dibenzene $2 f$}<smiles>CC(C)(C)c1ccc(C2(c3ccccc3)C(c3ccccc3)=C2c2ccccc2)cc1</smiles>

$2 f$

(Purple LED lamp, $405 \mathrm{~nm}$ ). Purified by flash column chromatography (100:0-99:1 hexanes/EtOAc). Isolated as a white solid (20 mg, 50\%). mp: 66-68 ${ }^{\circ} \mathrm{C}$. ${ }^{1} \mathrm{H}$ NMR $\left(500 \mathrm{MHz}, \mathrm{CDCl}_{3}\right) \delta(\mathrm{ppm}):$ 7.80-7.79 (m, 4H), 7.52-7.49 (m, 4H), 7.46-7.43 (m, 4H), 7.31-7.30 (m, 2H), $1.28(\mathrm{~s}, 9 \mathrm{H}) .{ }^{13} \mathrm{C}\left\{{ }^{1} \mathrm{H}\right\} \mathrm{NMR}\left(126 \mathrm{MHz}, \mathrm{CDCl}_{3}\right) \delta(\mathrm{ppm}): 150.0,134.4$, $131.8,130.2,129.9,129.2,127.5,127.2$ (q, $J=280.2 \mathrm{~Hz}), 126.5,125.5,110.3$ (q, J = 2.4 Hz), 34.5, 33.5 (q, J = 34.9 
$\mathrm{Hz}), 31.4 .{ }^{19} \mathrm{~F} \mathrm{NMR}\left(470 \mathrm{MHz}, \mathrm{CDCl}_{3}\right) \delta(\mathrm{ppm}):-62.1 . \mathrm{IR}\left(\mathrm{cm}^{-1}\right): 2961,1499,1447,1163,1124,926,754,687 . \mathrm{HR}-$ MS (EI) $\mathrm{m} / \mathrm{z}\left[\mathrm{M}+\mathrm{NH}_{4}\right]^{+}$calcd. for $\mathrm{C}_{26} \mathrm{H}_{27} \mathrm{NF}_{3} 410.2090$ found 410.2058 .

\section{(3-(4-Methoxyphenyl)-3-(trifluoromethyl)cycloprop-1-ene-1,2-diyl)dibenzene 2g}

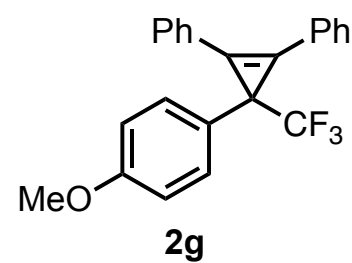

(Purple LED lamp, $405 \mathrm{~nm}$ ). Purified by flash column chromatography (100:0-99:1 hexanes/EtOAc). Isolated as a white solid (19 mg, 51\%). Exhibited spectral data identical to a previous report. ${ }^{11}{ }^{1} \mathrm{H}$ NMR $\left(500 \mathrm{MHz}, \mathrm{CDCl}_{3}\right) \delta$ (ppm): 7.80-7.78 (m, 4H), 7.53-7.49 $(\mathrm{m}, 4 \mathrm{H}), 7.47-7.42(\mathrm{~m}, 4 \mathrm{H}), 6.85-6.83(\mathrm{~m}, 2 \mathrm{H}), 3.77(\mathrm{~s}, 3 \mathrm{H}) .{ }^{13} \mathrm{C}\left\{{ }^{1} \mathrm{H}\right\} \mathrm{NMR}(126 \mathrm{MHz}$, $\left.\mathrm{CDCl}_{3}\right) \delta(\mathrm{ppm}): 158.8,130.1,129.9,129.4,129.3,129.1,129.0,127.3(\mathrm{q}, J=278.6 \mathrm{~Hz}), 126.5,114.0,110.6(\mathrm{q}, J=$ $2.4 \mathrm{~Hz}), 55.3,33.2(\mathrm{q}, \mathrm{J}=34.9 \mathrm{~Hz}) .{ }^{19} \mathrm{~F} \mathrm{NMR}\left(470 \mathrm{MHz}, \mathrm{CDCl}_{3}\right) \delta(\mathrm{ppm}):-62.4$.

\section{(3-(Trifluoromethyl)cycloprop-1-ene-1,2,3-triyl)tribenzene $2 \mathrm{~h}$}

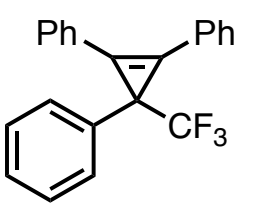

$2 \mathrm{~h}$

(UV LED lamp, $380 \mathrm{~nm}$ ). Purified by flash column chromatography (100:0-99:1 hexanes/EtOAc). Isolated as a white solid (4 mg, 12\%). Exhibited spectral data identical to a previous report. ${ }^{11}{ }^{1} \mathrm{H}$ $\operatorname{NMR}\left(500 \mathrm{MHz}, \mathrm{CDCl}_{3}\right) \delta(\mathrm{ppm}):$ 7.78-7.76 (m, 4H), 7.51-7.48 (m, 6H), 7.45-7.41 (m, 2H), 7.32$7.25(\mathrm{~m}, 2 \mathrm{H}), 7.25-7.21(\mathrm{~m}, 1 \mathrm{H}) .{ }^{13} \mathrm{C}\left\{{ }^{1} \mathrm{H}\right\} \mathrm{NMR}\left(126 \mathrm{MHz}, \mathrm{CDCl}_{3}\right) \delta(\mathrm{ppm}): 137.5,131.8,130.2$, 129.9, 129.3, 128.6, 127.7, 127.2, 127.3 (q, $J=278.6 \mathrm{~Hz}), 126.4,110.0$ (q, $J=2.4 \mathrm{~Hz}$ ), 33.8 (q, J = 34.9 Hz). ${ }^{19} \mathrm{~F} \mathrm{NMR}$ $\left(470 \mathrm{MHz}, \mathrm{CDCl}_{3}\right) \delta(\mathrm{ppm}):-62.4$.

\section{(3-(4-Fluorophenyl)-3-(trifluoromethyl)cycloprop-1-ene-1,2-diyl)dibenzene 2i}<smiles>Fc1ccc(C2(c3ccccc3)C(c3ccccc3)=C2c2ccccc2)cc1</smiles>

2i

(UV LED lamp, $380 \mathrm{~nm}$ ). Purified by flash column chromatography (100:0-99:1 hexanes/EtOAc). Isolated as a white solid (12 mg, 34\%). Exhibited spectral data identical to a previous report. ${ }^{11}{ }^{1} \mathrm{H}$ NMR $\left(500 \mathrm{MHz}, \mathrm{CDCl}_{3}\right) \delta(\mathrm{ppm}): 7.77-7.75(\mathrm{~m}, 4 \mathrm{H}), 7.52-7.49(\mathrm{~m}, 4 \mathrm{H})$, 7.46-7.42 (m, 4H), 6.99-6.95 (m, 2H). ${ }^{13} \mathrm{C}\left\{{ }^{1} \mathrm{H}\right\}$ NMR (126 MHz, CDCl 3$) \delta(\mathrm{ppm}): 161.9$ (d, $J=$ $277.9 \mathrm{~Hz}), 133.2,131.6,130.0,129.9,129.3,129.2,126.0,126.9$ (q, J = 279.6 Hz), 115.3 (d, J = 21.4 Hz), 110.1 (q, J $=2.3 \mathrm{~Hz}), 33.1$ (q, $J=35.2 \mathrm{~Hz}) .{ }^{19} \mathrm{~F} \mathrm{NMR}\left(470 \mathrm{MHz}, \mathrm{CDCl}_{3}\right) \delta$ (ppm): -62.3, -115.4.

\section{(3-(4-Bromophenyl)-3-(trifluoromethyl)cycloprop-1-ene-1,2-diyl)dibenzene 2j}

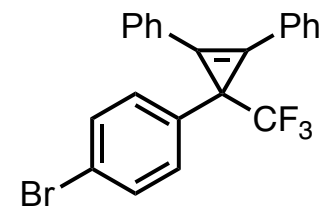

2j

(UV LED lamp, $380 \mathrm{~nm}$ ). Purified by flash column chromatography (100:0-99:1 hexanes/EtOAc). Isolated as a white solid (15 mg, 37\%). mp: 48-50 ${ }^{\circ} \mathrm{C} .{ }^{1} \mathrm{H} \mathrm{NMR}(500 \mathrm{MHz}$, $\left.\mathrm{CDCl}_{3}\right) \delta(\mathrm{ppm}):$ 7.75-7.74 (m, 4H), 7.52-7.49 (m, 4H), 7.46-7.43 (m, 2H), 7.41-7.36 (m, 4H). ${ }^{13} \mathrm{C}\left\{{ }^{1} \mathrm{H}\right\}$ NMR $\left(126 \mathrm{MHz}, \mathrm{CDCl}_{3}\right) \delta(\mathrm{ppm}): 136.6,131.8,130.2,130.1,129.4,129.3, \mathrm{j} 127.2$ (q, $J=275.7 \mathrm{~Hz}), 126.7,125.9,121.2,109.5,33.5$ (q, $J=34.9 \mathrm{~Hz}) .{ }^{19} \mathrm{~F} \mathrm{NMR}\left(470 \mathrm{MHz}, \mathrm{CDCl}_{3}\right) \delta(\mathrm{ppm}):-62.1 . \mathrm{IR}\left(\mathrm{cm}^{-1}\right)$ : 2924, 1489, 1445, 1296, 1165, 1109, 920, 827, 754, 689. HR-MS (El) m/z [M + H] calcd. for $\mathrm{C}_{22} \mathrm{H}_{15} \mathrm{~F}_{3} \mathrm{Br} 415.0304$ found 415.0349 . 
(3-(4-Bromophenyl)-3-(trifluoromethyl)cycloprop-1-ene-1,2-diyl)dibenzene 2k<smiles>FC(F)(F)C1(c2cccc(Br)c2)C(c2ccccc2)=C1c1ccccc1</smiles>

2k

(UV LED lamp, $380 \mathrm{~nm}$ ). Purified by flash column chromatography (100:0-99:1 hexanes/EtOAc). Isolated as a white semi solid (10 mg, 24\%). ${ }^{1} \mathrm{H}$ NMR $\left(500 \mathrm{MHz}, \mathrm{CDCl}_{3}\right) \delta$ (ppm): 7.76-7.74 (m, 4H), 7.64 (s, 1H), 7.53-7.50 (m, 4H), 7.46-7.43 (m, 2H) 7.42-7.34 (m, 2H), 7.16-7.13 (m, 1H). ${ }^{13} \mathrm{C}\left\{{ }^{1} \mathrm{H}\right\} \mathrm{NMR}\left(126 \mathrm{MHz}, \mathrm{CDCl}_{3}\right) \delta(\mathrm{ppm}): 140.0,131.8,130.6,130.4$, 130.2, 130.2, 130.1, 129.4, 127.1 (q, $J=278.9 \mathrm{~Hz}), 125.8,122.8,109.4$ (q, $J=2.6 \mathrm{~Hz}), 33.5$ (q, $J=35.1 \mathrm{~Hz}) .{ }^{19} \mathrm{~F} \mathrm{NMR}$ (470 MHz, $\left.\mathrm{CDCl}_{3}\right) \delta$ (ppm): -61.9. IR (cm-1): 2924, 1562, 1296, 1163, 1122, 935, 754, 685. HR-MS (EI) m/z [M + H] ${ }^{+}$ calcd. for $\mathrm{C}_{22} \mathrm{H}_{15} \mathrm{~F} 3 \mathrm{Br} 415.0304$ found 415.0373 .

\section{(3-(4-Trifluoromethylphenyl)-3-(trifluoromethyl)cycloprop-1-ene-1,2-diyl)dibenzene 2I}<smiles>FC(F)(F)c1ccc(C2(c3ccccc3)C(c3ccccc3)=C2c2ccccc2)cc1</smiles>

2I

(UV LED lamp, $380 \mathrm{~nm}$ ). Purified by flash column chromatography (100:0-99:1 hexanes/EtOAc). Isolated as a white solid (20 mg, 51\%). mp: $40-42{ }^{\circ} \mathrm{C}$. ${ }^{1} \mathrm{H}$ NMR $\left(500 \mathrm{MHz}, \mathrm{CDCl}_{3}\right) \delta(\mathrm{ppm}):$ 7.76-7.74 (m, 1H), 7.56-7.54 (m, 4H), 7.53-7.50 (m, 2H) 7.39-7.34 (m, 7H). ${ }^{13} \mathrm{C}\left\{{ }^{1} \mathrm{H}\right\} \mathrm{NMR}\left(126 \mathrm{MHz}, \mathrm{CDCl}_{3}\right) \delta(\mathrm{ppm}): 131.8,130.1,129.4,128.5$, 128.4, $126.8(q, J=278.8 \mathrm{~Hz}), 126.6,125.5$ (q, $J=3.8 \mathrm{~Hz}), 124.2$ (q, $J=271.8 \mathrm{~Hz}), 123.4,109.1$ (q, J=2.1 Hz), 89.5, $33.7(\mathrm{q}, \mathrm{J}=35.0 \mathrm{~Hz}){ }^{19} \mathrm{~F}$ NMR $\left(470 \mathrm{MHz}, \mathrm{CDCl}_{3}\right) \delta(\mathrm{ppm}):-61.9,-62.6 . \mathrm{IR}\left(\mathrm{cm}^{-1}\right): 3065,1495,1443,1325,1163$, $1119,1067,918,749,685$. HR-MS (EI) m/z [M + H] calcd. for $\mathrm{C}_{23} \mathrm{H}_{15} \mathrm{~F}_{6} 405.1078$ found 405.1072 .

\subsection{General procedure for the photochemical reactions of diazirines in batch}

A solution of the diazirine 1 and alkyne (10 equiv) in $\mathrm{CH}_{2} \mathrm{Cl}_{2}(0.4 \mathrm{M})$ were stirred under purple LED irradiation (See below). Reactions were monitored by TLC. After $24 \mathrm{~h}$ reactions were still incomplete. The solvent was removed under reduced pressure and 3-fluoro-4-nitrotoluene was added as an internal standard for yield calculation by ${ }^{19 F}$ NMR.
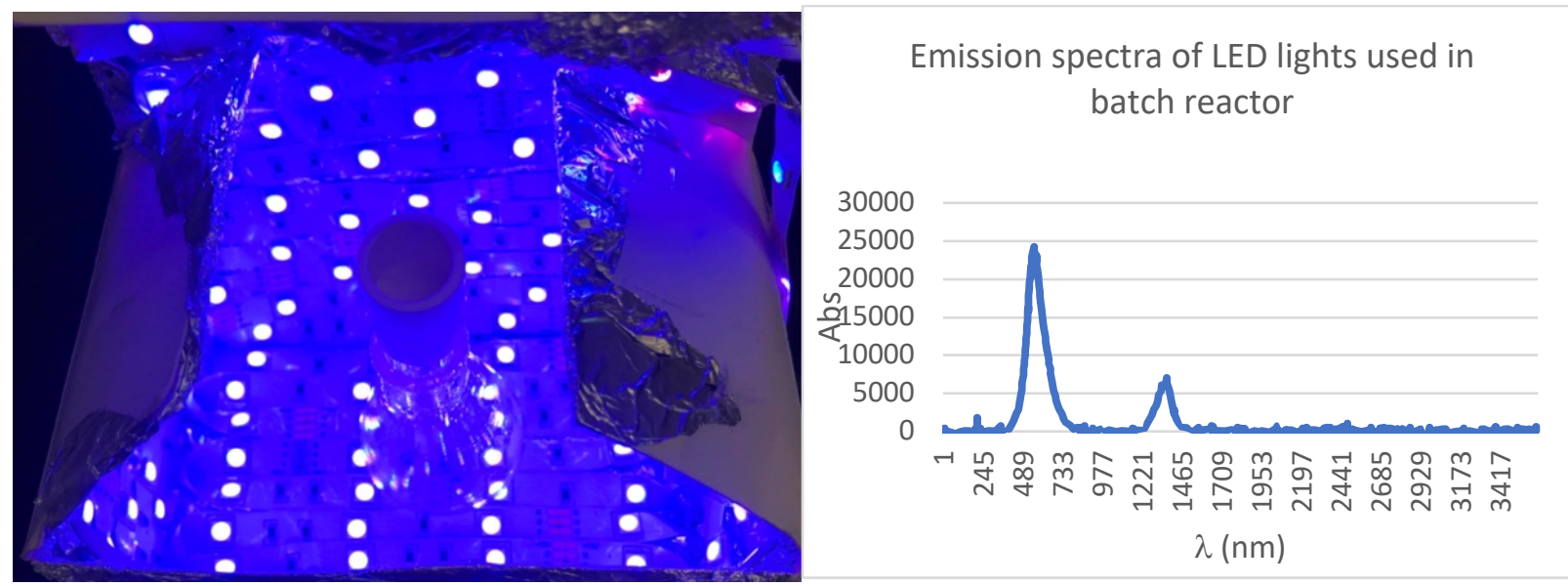

Figure S3. Batch setup with LED light strips 


\subsection{Competition experiments}

\subsubsection{General procedure for the competition reaction between internal and terminal alkynes}

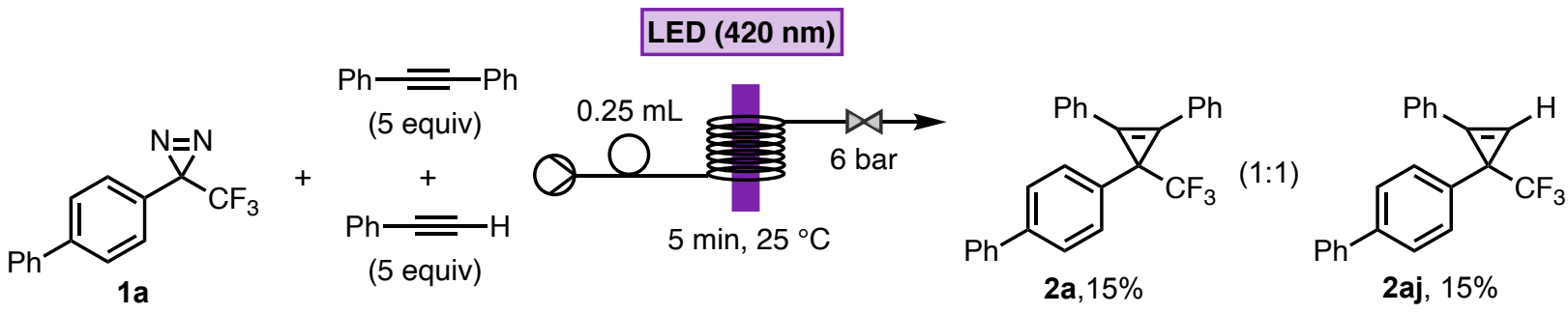

$0.4 \mathrm{M}$ in $\mathrm{CH}_{2} \mathrm{Cl}_{2}$

The general flow procedure described in $\mathbf{5 . 2}$ was used. A solution of the diazirine 1a, diphenyl acetylene ( 5 equiv), and phenyl acetylene (5 equiv) in $\mathrm{CH}_{2} \mathrm{Cl}_{2}(0.4 \mathrm{M}$ to $1 \mathrm{a})$ was prepared and loaded into the injection loop $(0.25 \mathrm{~mL}$, reaction scale $=0.1 \mathrm{mmol}$ ). The flow rate was set to $2 \mathrm{~mL} / \mathrm{min}$ for a $10 \mathrm{~mL}$ reactor volume (residence time $=5 \mathrm{~min}$ ). The flow reactions were run under LED irradiation $(420 \mathrm{~nm})$ at $25{ }^{\circ} \mathrm{C}$. The post-reaction stream containing the desired cyclopropene product (pre- and post-slug volume $=1 \mathrm{~mL}$ ) was collected in a $30 \mathrm{~mL}$ vial. The solvent was removed under reduced pressure. The crude mixture was analyzed by ${ }^{19} \mathrm{~F}$ NMR using 3-fluoro-4-nitrotoluene as an internal standard (-117.83 ppm). The obtained spectra in displayed below:

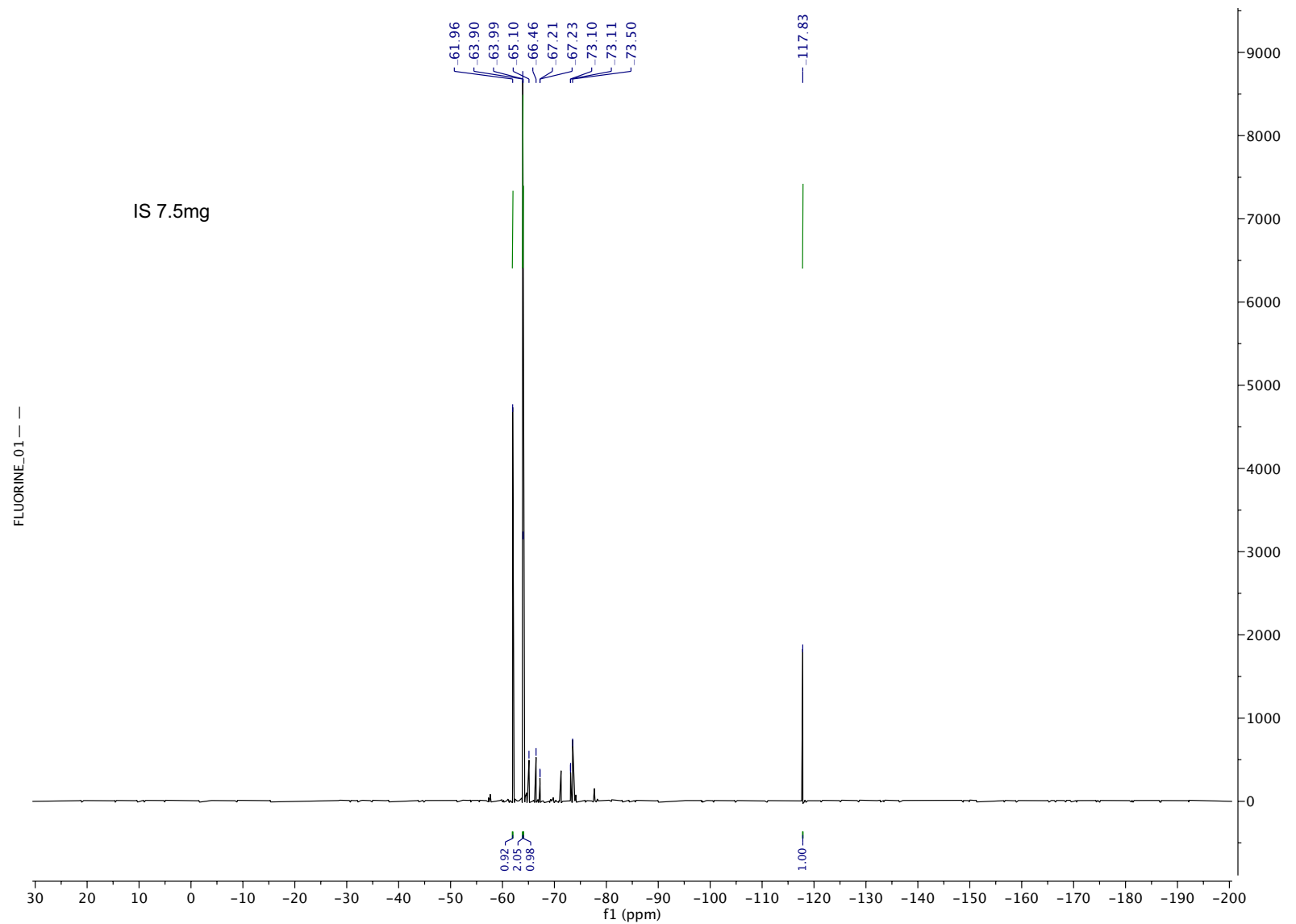




\subsubsection{General procedure for the competition reaction between internal alkynes and alkenes}

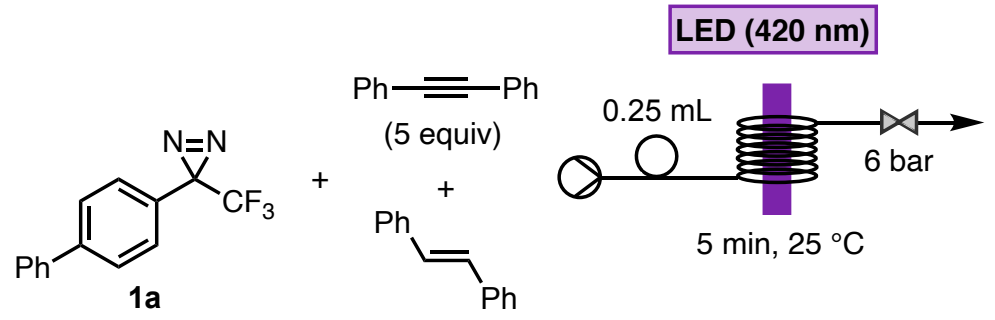<smiles>FC(F)(F)C1(c2ccccc2)C(c2ccccc2)=C1c1ccccc1</smiles>

2a, $45 \%$
$(1.25: 1)$<smiles>FC(F)(F)c1ccc(-c2ccccc2)cc1C1C(c2ccccc2)C1c1ccccc1</smiles>

$2 a^{\prime}, 36 \%$

$0.4 \mathrm{M}$ in $\mathrm{CH}_{2} \mathrm{Cl}_{2} \quad$ (5 equiv)

The general flow procedure described in 5.2 was used. A solution of the diazirine $1 \mathrm{a}$, diphenyl acetylene ( 5 equiv), and (E)-1,2-diphenylethene (5 equiv) in $\mathrm{CH}_{2} \mathrm{Cl}_{2}(0.4 \mathrm{M}$ to $1 \mathrm{a})$ was prepared and loaded into the injection loop ( $0.25 \mathrm{~mL}$, reaction scale $=0.1 \mathrm{mmol}$ ). The flow rate was set to $2 \mathrm{~mL} / \mathrm{min}$ for a $10 \mathrm{~mL}$ reactor volume (residence time $=5 \mathrm{~min}$ ). The flow reactions were run under LED irradiation $(420 \mathrm{~nm})$ at $25^{\circ} \mathrm{C}$. The post-reaction stream containing the desired cyclopropene product (pre- and post-slug volume $=1 \mathrm{~mL}$ ) was collected in a $30 \mathrm{~mL}$ vial. The solvent was removed under reduced pressure. The crude mixture was analyzed by ${ }^{19} \mathrm{~F}$ NMR using 3-fluoro-4-nitrotoluene as an internal standard (-117.83 ppm). The obtained spectra in displayed below:

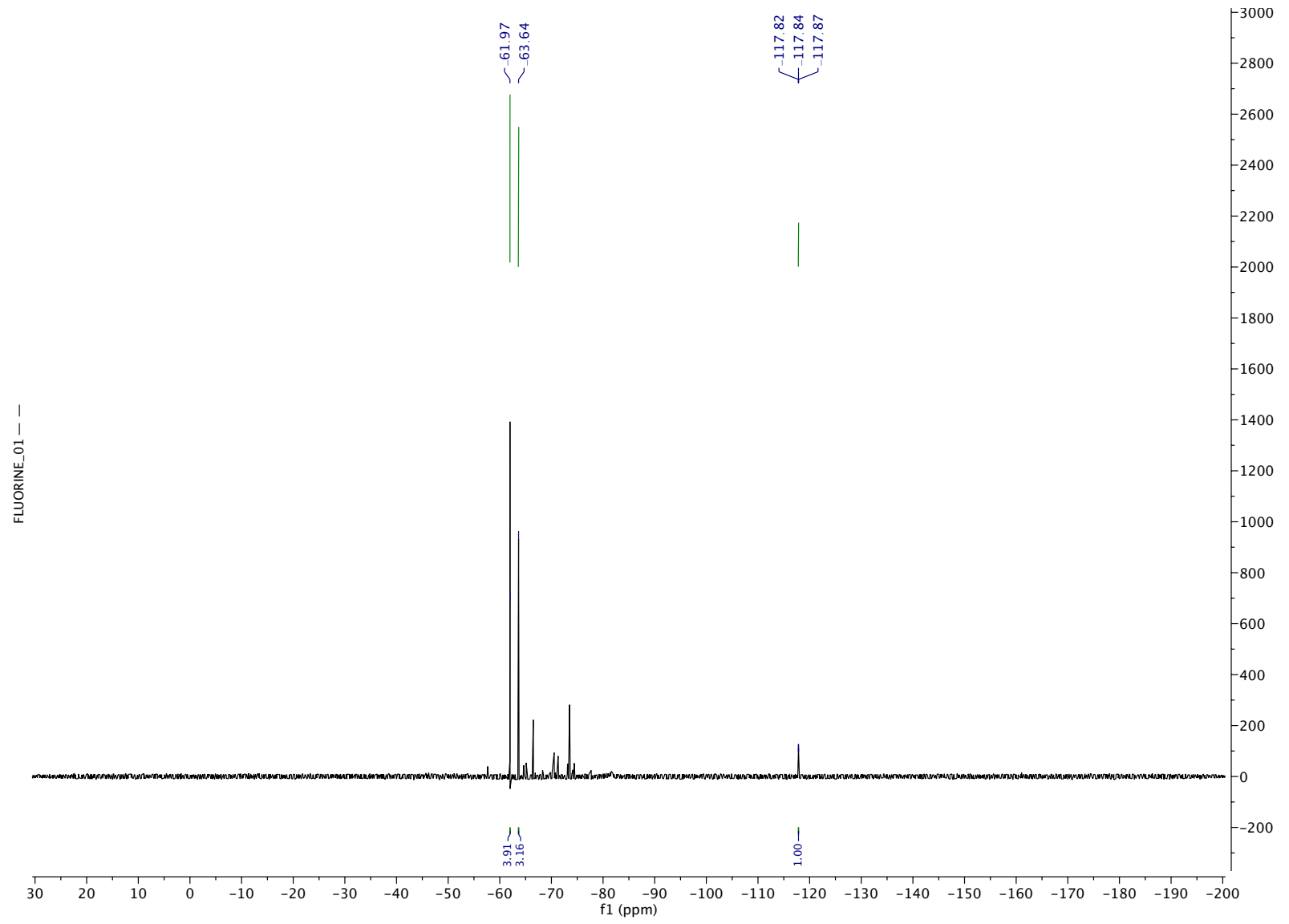




\subsubsection{General procedure for the competition reaction between internal alkynes and alkenes}

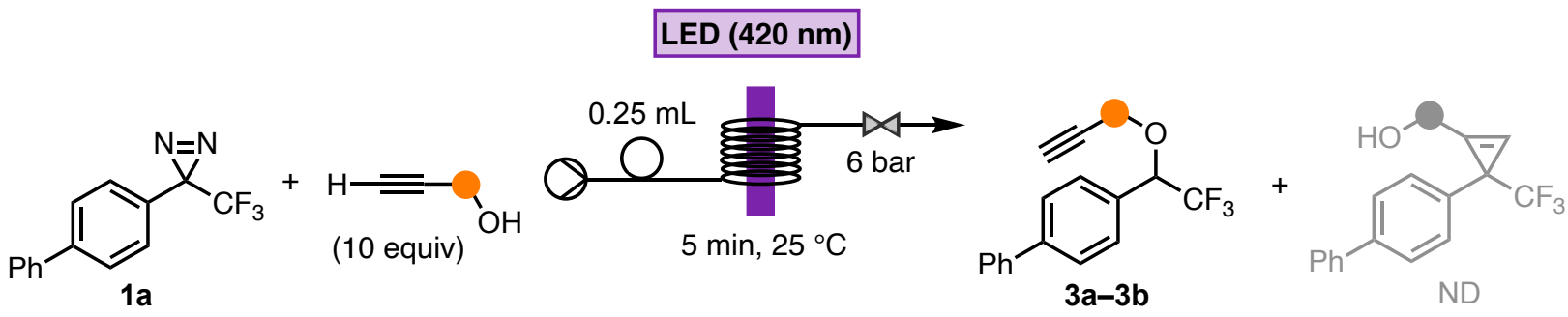

$0.4 \mathrm{M}$ in $\mathrm{CH}_{2} \mathrm{Cl}_{2}$

The general flow procedure described in $\mathbf{5 . 2}$ was used. A solution of the diazirine 1a and the propargyl alcohol (10 equiv.) in $\mathrm{CH}_{2} \mathrm{Cl}_{2}(0.4 \mathrm{M}$ to $1 \mathrm{a})$ was prepared and loaded into the injection loop $(0.25 \mathrm{~mL}$, reaction scale $=0.1 \mathrm{mmol})$. The flow rate was set to $2 \mathrm{~mL} / \mathrm{min}$ for a $10 \mathrm{~mL}$ reactor volume (residence time $=5 \mathrm{~min}$ ). The flow reactions were run under LED irradiation $(420 \mathrm{~nm})$ at $25^{\circ} \mathrm{C}$. The post-reaction stream containing the desired cyclopropene product (preand post-slug volume $=1 \mathrm{~mL}$ ) was collected in a $30 \mathrm{~mL}$ vial. The solvent was removed under reduced pressure and the crude mixture was purified by flash column chromatography (Biotage, SNAP ULTRA, 100-7/3 hexanes:EtOAc) to give the compounds $3 \mathbf{a}$ and $\mathbf{3 b}$.

\subsubsection{Characterization of 2,2,2-trifluoromethyl ether 3a and 3b}

4-(2,2,2-Trifluoro-1-(pent-4-yn-1-yloxy)ethyl)-1,1'-biphenyl 3a<smiles>C#CCCCOC(c1ccc(-c2ccccc2)cc1)C(F)(F)F</smiles>

Purified by flash column chromatography (100:0-70:30 hex/EtOAc). Isolated as a colorless oil (18 mg, 58\%). ${ }^{1} \mathrm{H} \mathrm{NMR}\left(500 \mathrm{MHz}, \mathrm{CDCl}_{3}\right) \delta(\mathrm{ppm}): 7.63(\mathrm{~m}, 4 \mathrm{H}), 7.52(\mathrm{~d}, \mathrm{~J}=8.5 \mathrm{~Hz}, 2 \mathrm{H})$, $7.51-7.44(\mathrm{~m}, 2 \mathrm{H}), 7.42-7.35(\mathrm{~m}, 1 \mathrm{H}), 4.67(\mathrm{q}, J=6.6 \mathrm{~Hz}, 1 \mathrm{H}), 3.67(\mathrm{td}, J=6.0,3.2 \mathrm{~Hz}$, 2H), 2.37 (tdd, $J=7.0,5.8,2.7 \mathrm{~Hz}, 2 \mathrm{H}), 1.94(\mathrm{t}, J=2.6 \mathrm{~Hz}, 1 \mathrm{H}), 1.91-1.81(\mathrm{~m}, 2 \mathrm{H}) .{ }^{13} \mathrm{C}\left\{{ }^{1} \mathrm{H}\right\}$ $\operatorname{NMR}\left(126 \mathrm{MHz}, \mathrm{CDCl}_{3}\right) \delta(\mathrm{ppm}): 142.4,140.4,131.8,128.9,128.6,127.6,127.3,127.2,122.7$ (q, J = $\left.259.4 \mathrm{~Hz}\right), 83.5$, 79.9 (q, J = 31.1 Hz), 69.0,68.8, 28.5, 15.1. ${ }^{19} \mathrm{~F} \mathrm{NMR}\left(470 \mathrm{MHz}, \mathrm{CDCl}_{3}\right) \delta(\mathrm{ppm}):-6.59$ (d, J=6.8 Hz). IR (cm-1): 3289 , 2915, 1489, 1370, 1270, 1174, 1135, 1095, 1011, 763, 693. HR-MS (El) m/z (compound did not ionize).

\section{4-(2,2,2-Trifluoro-1-(prop-2-yn-1-yloxy)ethyl)-1,1'-biphenyl 3b}<smiles>C#CCOC(c1ccc(-c2ccccc2)cc1)C(F)(F)F</smiles>

Purified by flash column chromatography (100:0-70:30 hex/EtOAc). Isolated as a white solid (20 mg, 70\%). mp: $48-50{ }^{\circ} \mathrm{C}{ }^{1} \mathrm{H} \mathrm{NMR}\left(500 \mathrm{MHz}, \mathrm{CDCl}_{3}\right) \delta$ (ppm): $7.70-7.63(\mathrm{~m}, 2 \mathrm{H}), 7.64$ $-7.58(\mathrm{~m}, 2 \mathrm{H}), 7.54(\mathrm{~d}, J=9.2 \mathrm{~Hz}, 2 \mathrm{H}), 7.53-7.43(\mathrm{~m}, 2 \mathrm{H}), 7.43-7.36(\mathrm{~m}, 1 \mathrm{H}), 5.02(\mathrm{q}, J$ $=6.7 \mathrm{~Hz}, 1 \mathrm{H}), 4.40(\mathrm{dd}, J=15.9,2.4 \mathrm{~Hz}, 1 \mathrm{H}), 4.13(\mathrm{dd}, J=16.0,2.4 \mathrm{~Hz}, 1 \mathrm{H}), 2.53(\mathrm{t}, J=2.4$ $\mathrm{Hz}, 1 \mathrm{H}) .{ }^{13} \mathrm{C}\left\{{ }^{1} \mathrm{H}\right\} \mathrm{NMR}\left(126 \mathrm{MHz}, \mathrm{CDCl}_{3}\right) \delta(\mathrm{ppm}): 142.8,140.3,130.5,128.9,128.9,127.7,127.5,127.2,124.8$ (q, J $=281.0 \mathrm{~Hz}$ ), 77.8, 77.4 (q, J = 31.8 Hz), 76.1, 56.7. $\left.{ }^{19} \mathrm{~F} \mathrm{NMR} \mathrm{(470} \mathrm{MHz,} \mathrm{CDCl}_{3}\right) \delta(\mathrm{ppm}):-76.05$ (d, J = 5.2 Hz). IR $\left(\mathrm{cm}^{-1}\right): 3288,2913,1489,1369,1259,1175,1134,1097,1018,764,692$. HR-MS (EI) m/z (compound did not ionize). 


\subsection{General procedure for the TMS deprotection of 2ak}

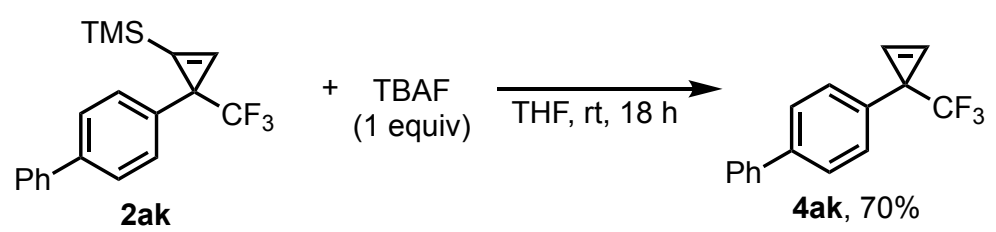

The cyclopropene $2 \mathrm{ak}(0.06 \mathrm{mmol}, 20 \mathrm{mg})$ was dissolved in $0.3 \mathrm{~mL}$ of anhydrous THF. TBAF (1 M in THF, $60 \mu \mathrm{L}, 1$ equiv) was added and the reaction mixture was stirred for $18 \mathrm{~h}$ at room temperature. The solvent was removed under reduced pressure and the residue was purified by flash column chromatography (Biotage, SNAP ULTRA, 100\% hexanes) to give the compound $\mathbf{4 a k}$.

\section{4-(1-(Trifluoromethyl)cycloprop-2-en-1-yl)-1,1'-biphenyl 4ak}<smiles>FC(F)(F)C1(c2ccc(-c3ccccc3)cc2)C=C1</smiles>

Isolated as a white solid (11 mg, 70\%). mp: 58-60 ${ }^{\circ} \mathrm{C}{ }^{1} \mathrm{H} \mathrm{NMR}\left(500 \mathrm{MHz}, \mathrm{CDCl}_{3}\right) \delta(\mathrm{ppm})$ : 7.59-7.55 (m, 4H), 7.46-7.43 (m, 2H), $7.39(\mathrm{~d}, \mathrm{~J}=8.0 \mathrm{~Hz}, 2 \mathrm{H}), 7.37-7.34(\mathrm{~m}, 1 \mathrm{H}), 7.29(\mathrm{q}, J$ $=1.5 \mathrm{~Hz}, 2 \mathrm{H}) .{ }^{13} \mathrm{C}\left\{{ }^{1} \mathrm{H}\right\} \mathrm{NMR}\left(126 \mathrm{MHz}, \mathrm{CDCl}_{3}\right) \delta(\mathrm{ppm}): 140.8,140.4,137.8,128.9,128.2$, 128.1, 127.5, 127.3, 127.1 (q, J = 276.6 Hz), 127.2, 108.0 (q, $J=2.8 \mathrm{~Hz}), 29.4$ (q, J = 36.0 $\mathrm{Hz}) .{ }^{19} \mathrm{~F} \mathrm{NMR}\left(470 \mathrm{MHz}, \mathrm{CDCl}_{3}\right) \delta(\mathrm{ppm}):-65.6 . \mathrm{IR}\left(\mathrm{cm}^{-1}\right): 3142,3032,1653,1487,1259,1119,831,764,735$. HR-MS (EI) m/z (compound did not ionize).

\subsection{Reaction monitoring using ReactIR}

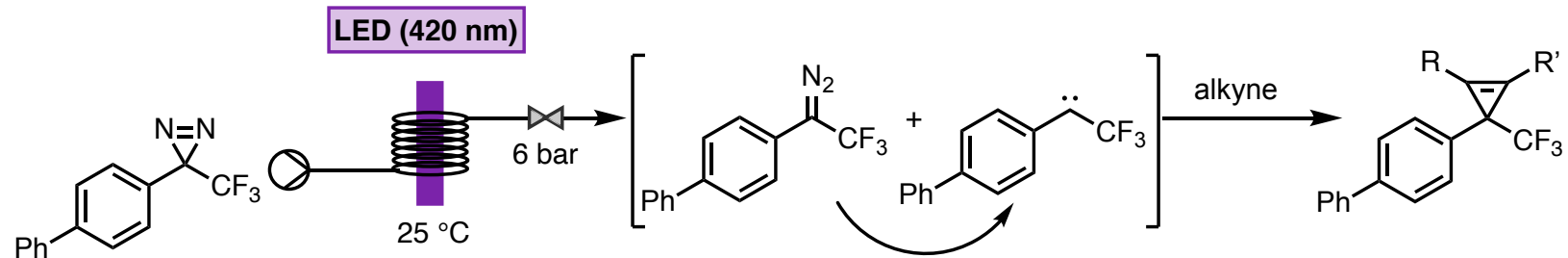

The general flow procedure described in $\mathbf{5 . 2}$ was used. A solution of the diazirine $1 \mathrm{a}$ and diphenyl acetylene (10 equiv) in $\mathrm{CH}_{2} \mathrm{Cl}_{2}(0.4 \mathrm{M}$ to $1 \mathrm{a})$ was prepared and loaded into the injection loop $(0.25 \mathrm{~mL}$, reaction scale $=0.1 \mathrm{mmol})$. The flow reactions were run under LED irradiation $(420 \mathrm{~nm})$ at $25^{\circ} \mathrm{C}$ in a $10 \mathrm{~mL}$ tube reactor. The outlet of the reactor was connected to the ReactIR using a silicon probe and reaction monitoring was done in real time. The flow rate was varied from 2 to $4 \mathrm{~mL} / \mathrm{min}$ for a better detection of reaction intermediates. The figure below is the setup used for this experiment: 


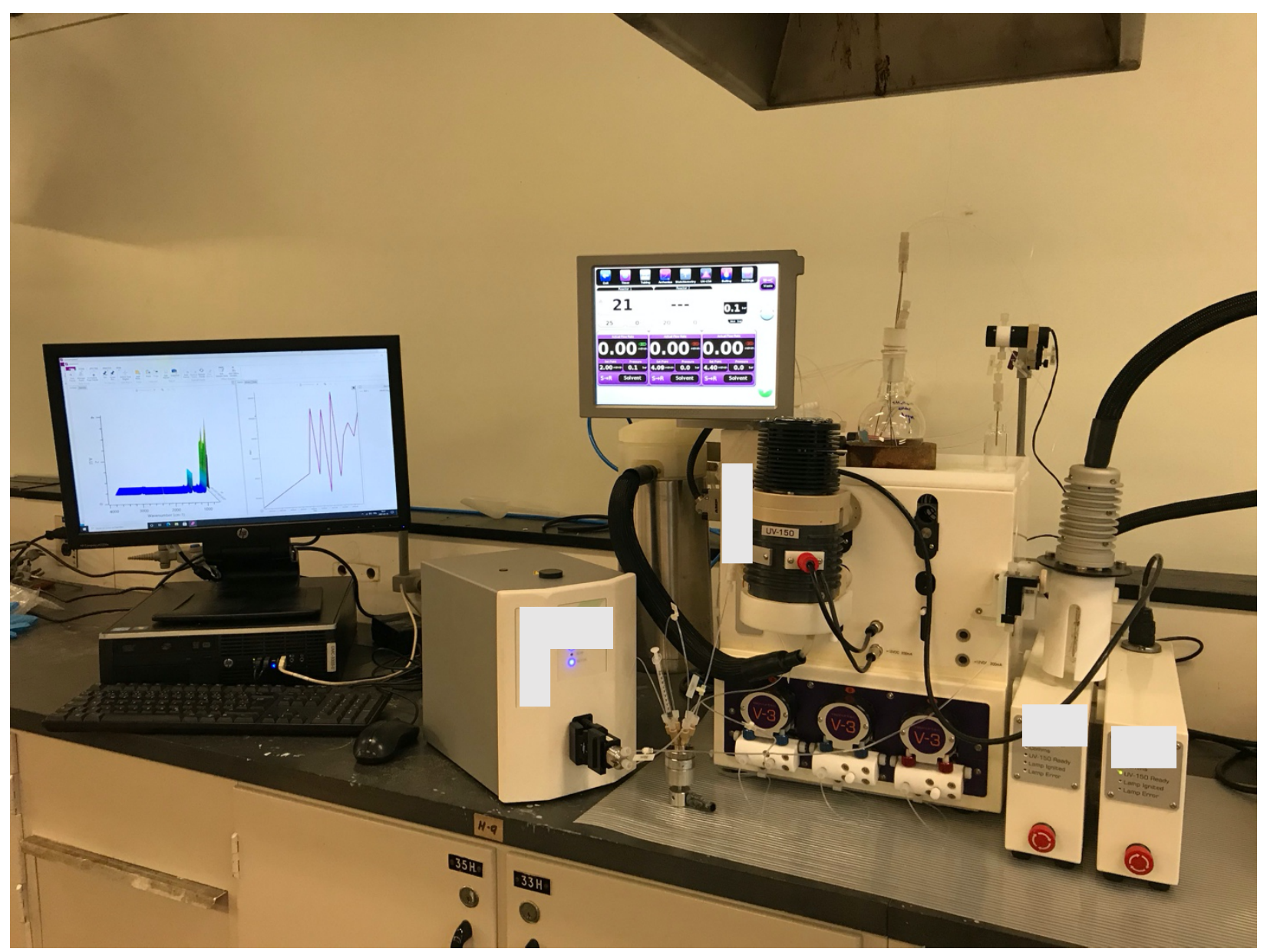

On the left: ReactIR equipement; On the right: flow setup

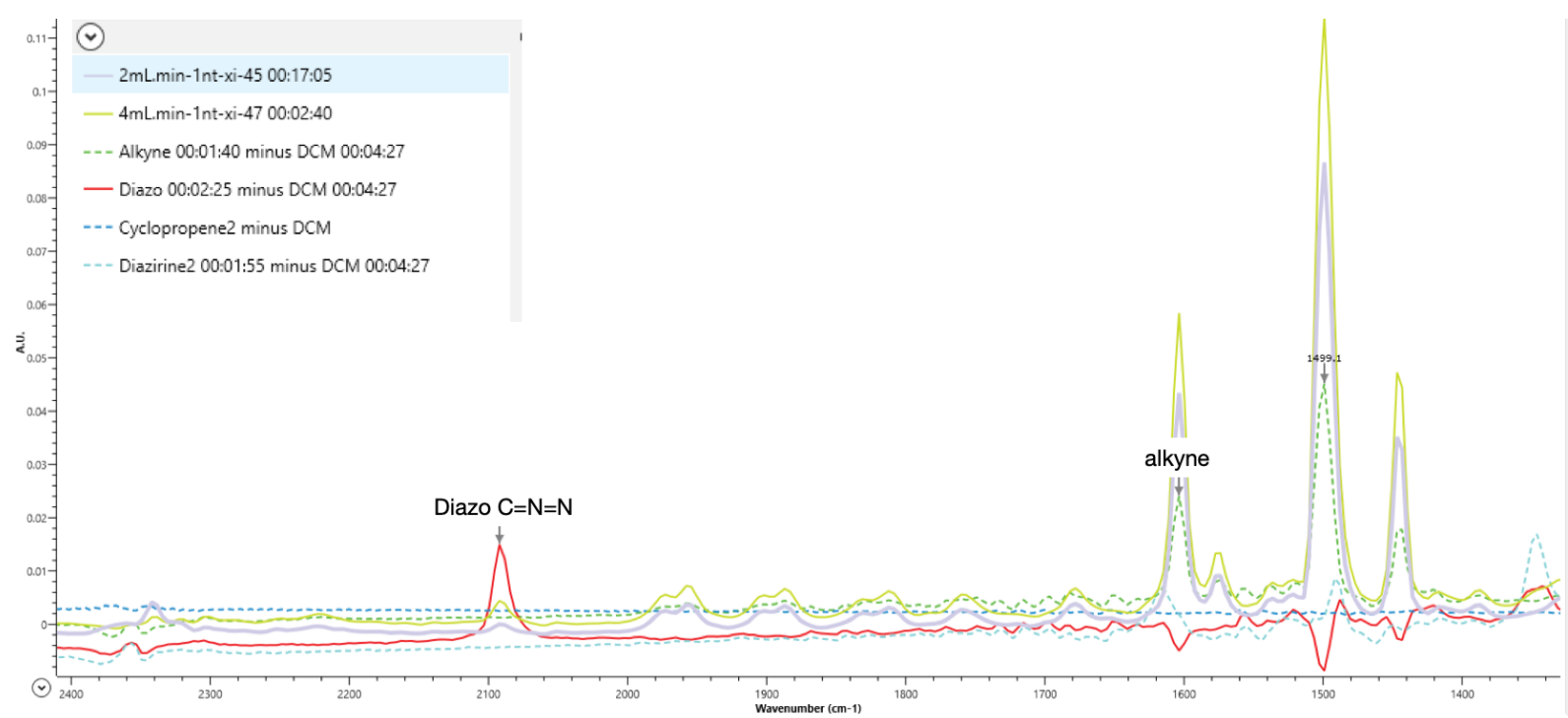

From the obtained IR spectrum, the characteristic peak of the diazo compound at $2100 \mathrm{~cm}^{-1}$ was observed with increased intensity when the flow rate was increased from 2 to $4 \mathrm{~mL} / \mathrm{min}$. 


\section{Control experiments}

A solution of cyclopropene $2 \mathrm{a}$ in $\mathrm{CH}_{2} \mathrm{Cl}_{2}(0.1 \mathrm{M})$ was prepared and loaded into the injection loop $(0.25 \mathrm{~mL})$. The flow rate was set to $2 \mathrm{~mL} / \mathrm{min}$ for a $10 \mathrm{~mL}$ reactor volume (residence time $=5 \mathrm{~min}$ ). The flow reactions were run under LED irradiation (A: $420 \mathrm{~nm}$ and $\mathrm{B}: 365 \mathrm{~nm}$ ) at $25^{\circ} \mathrm{C}$. The post-reaction stream containing the cyclopropene product (preand post-slug volume $=1 \mathrm{~mL}$ ) was collected in a $10 \mathrm{~mL}$ vial. The solvent was removed under reduced pressure. ${ }^{1} \mathrm{H}$ and ${ }^{19} \mathrm{~F}$ NMR of the mixture were recorded and compared to the starting material $2 \mathrm{a}$. The obtained spectra are displayed below (Stacked + individual spectra):
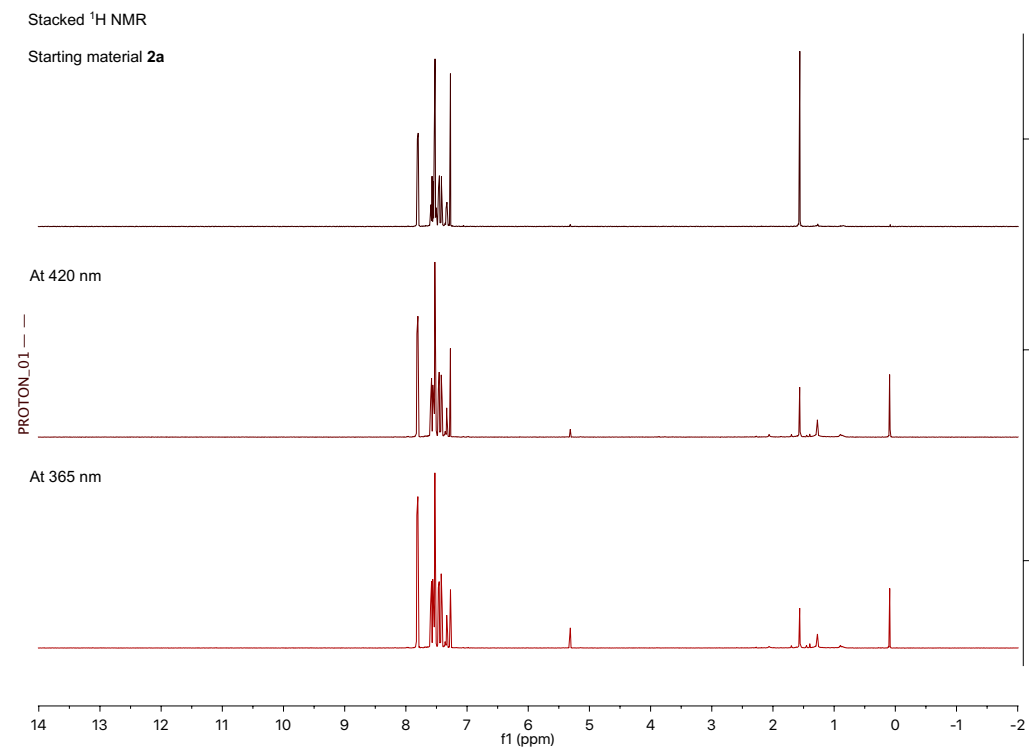

Stacked ${ }^{19} \mathrm{~F}$ NMR

Starting material 2a

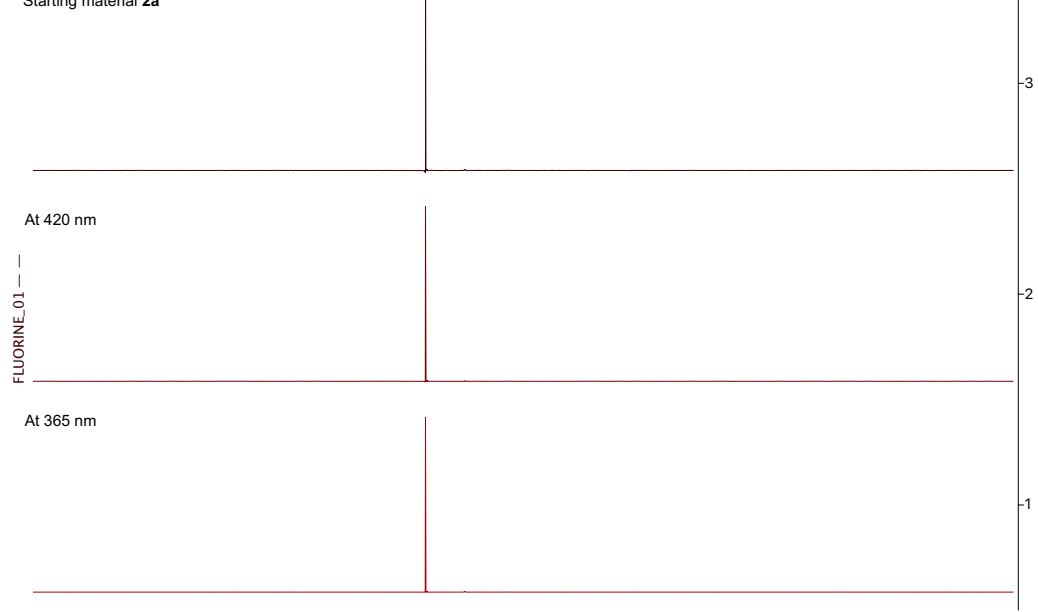

$\begin{array}{llllllllllllllllllllllllllll}30 & 20 & 10 & 0 & -10 & -20 & -30 & -40 & -50 & -60 & -70 & -80 & -90 & -100 & -110 & -120 & -130 & -140 & -150 & -160 & -170 & -180 & -190 & -200\end{array}$ 
${ }^{1} \mathrm{H}$ NMR spectra of $2 \mathrm{a}$ (Starting material)

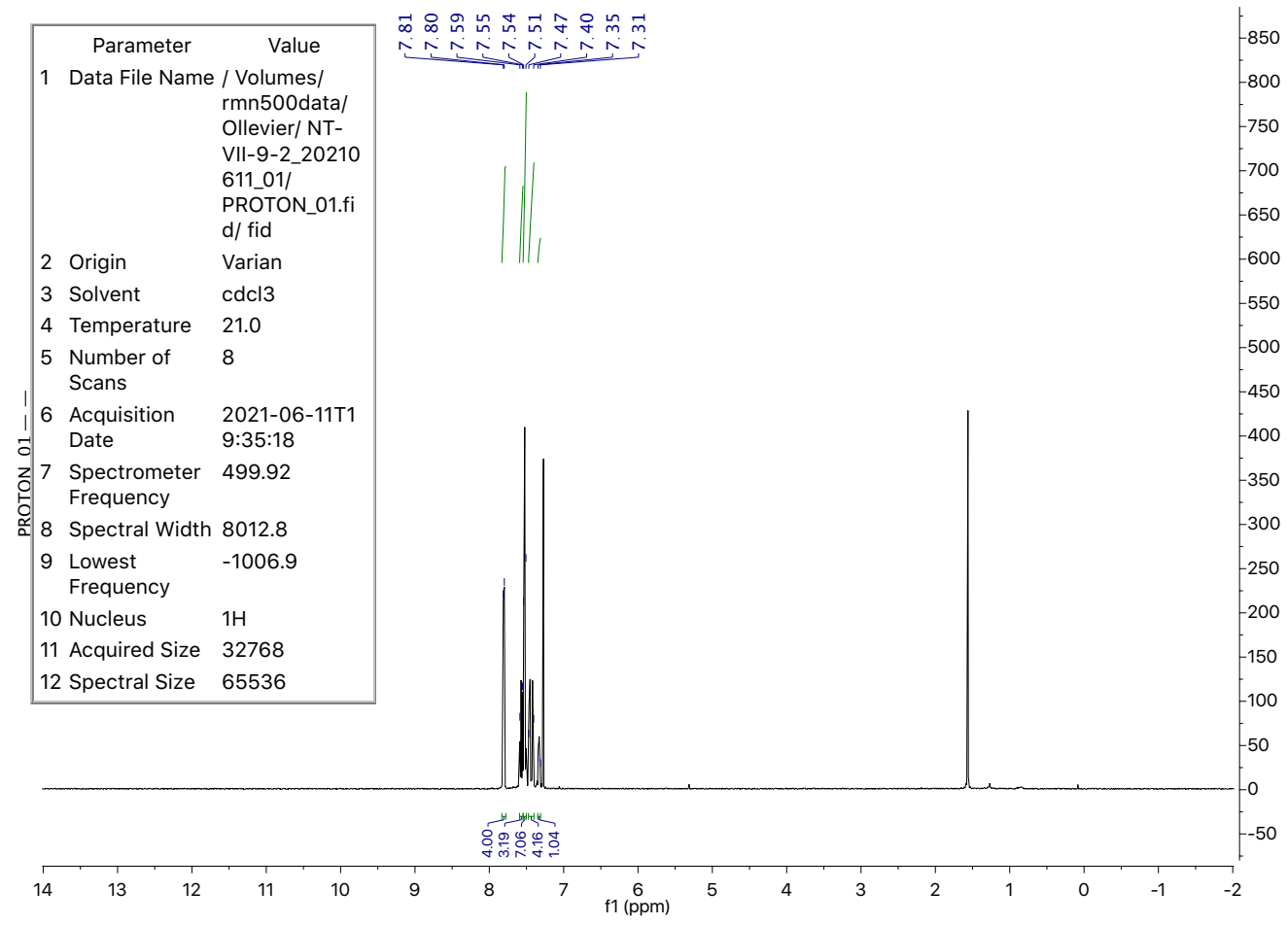

\section{${ }^{19} \mathrm{~F}$ NMR spectra of $2 a$ (Starting material)}

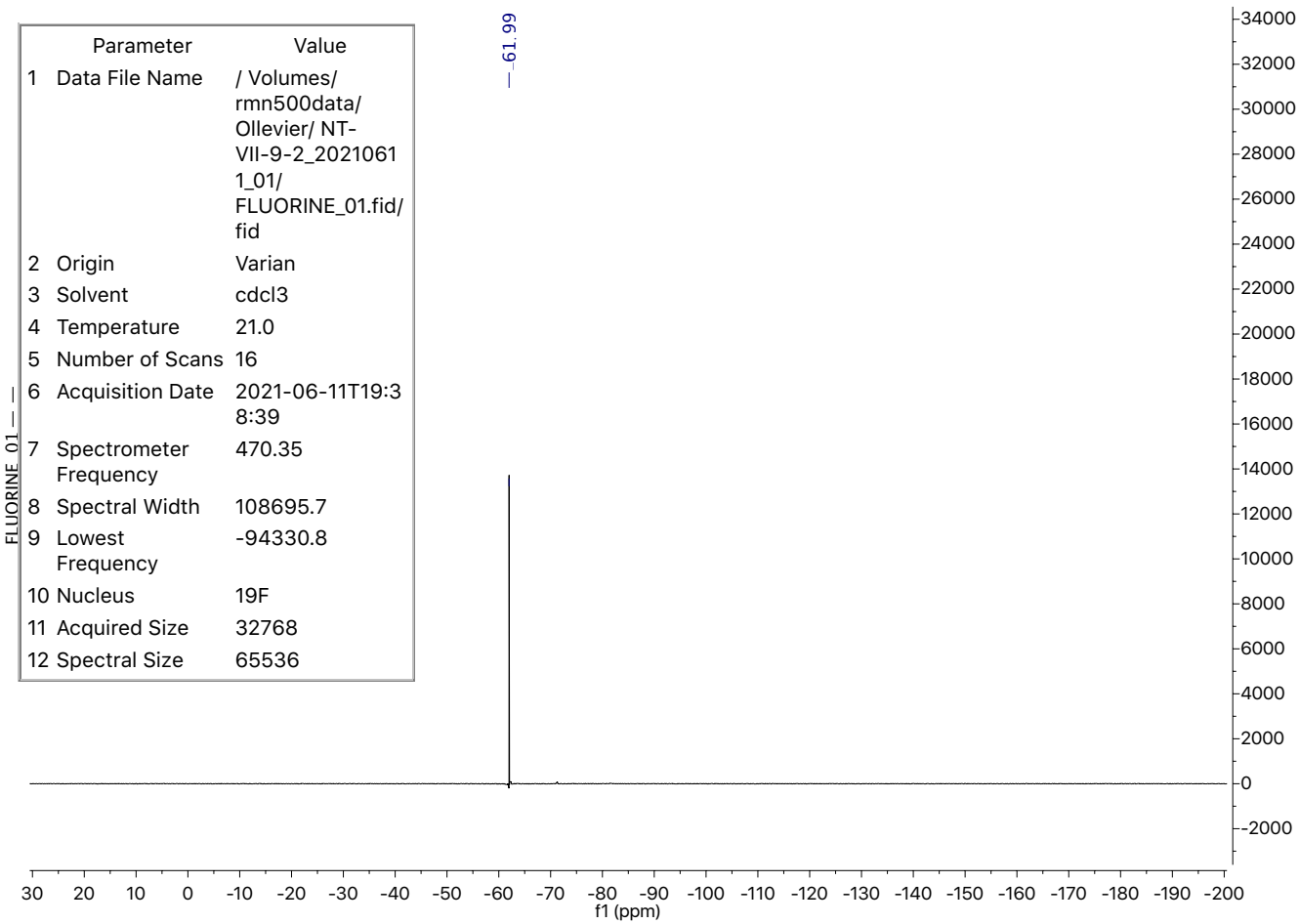


A: $420 \mathrm{~nm}$

${ }^{1} \mathrm{H}$ NMR spectra of the mixture

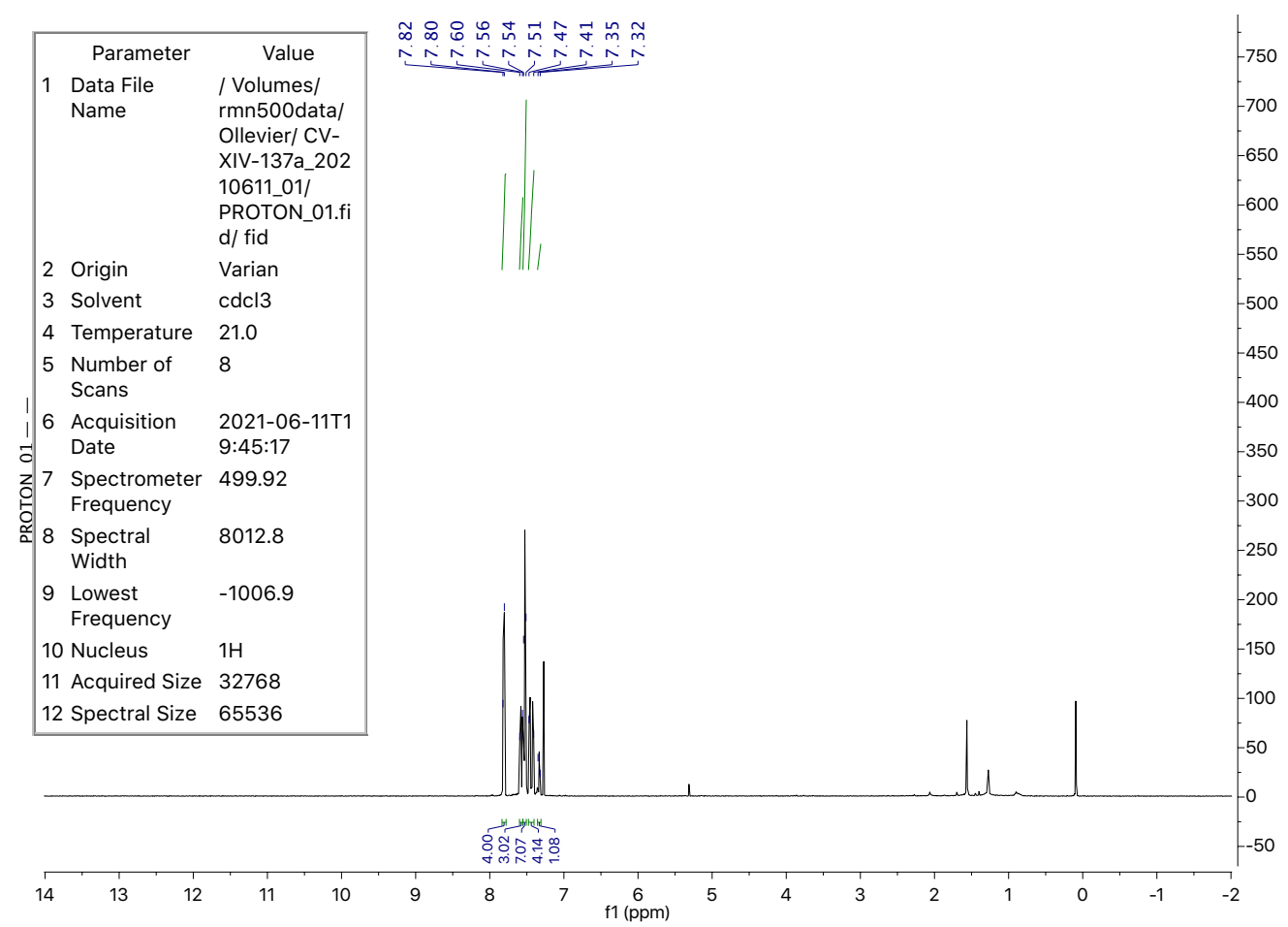

${ }^{19} \mathrm{~F}$ NMR spectra of the mixture

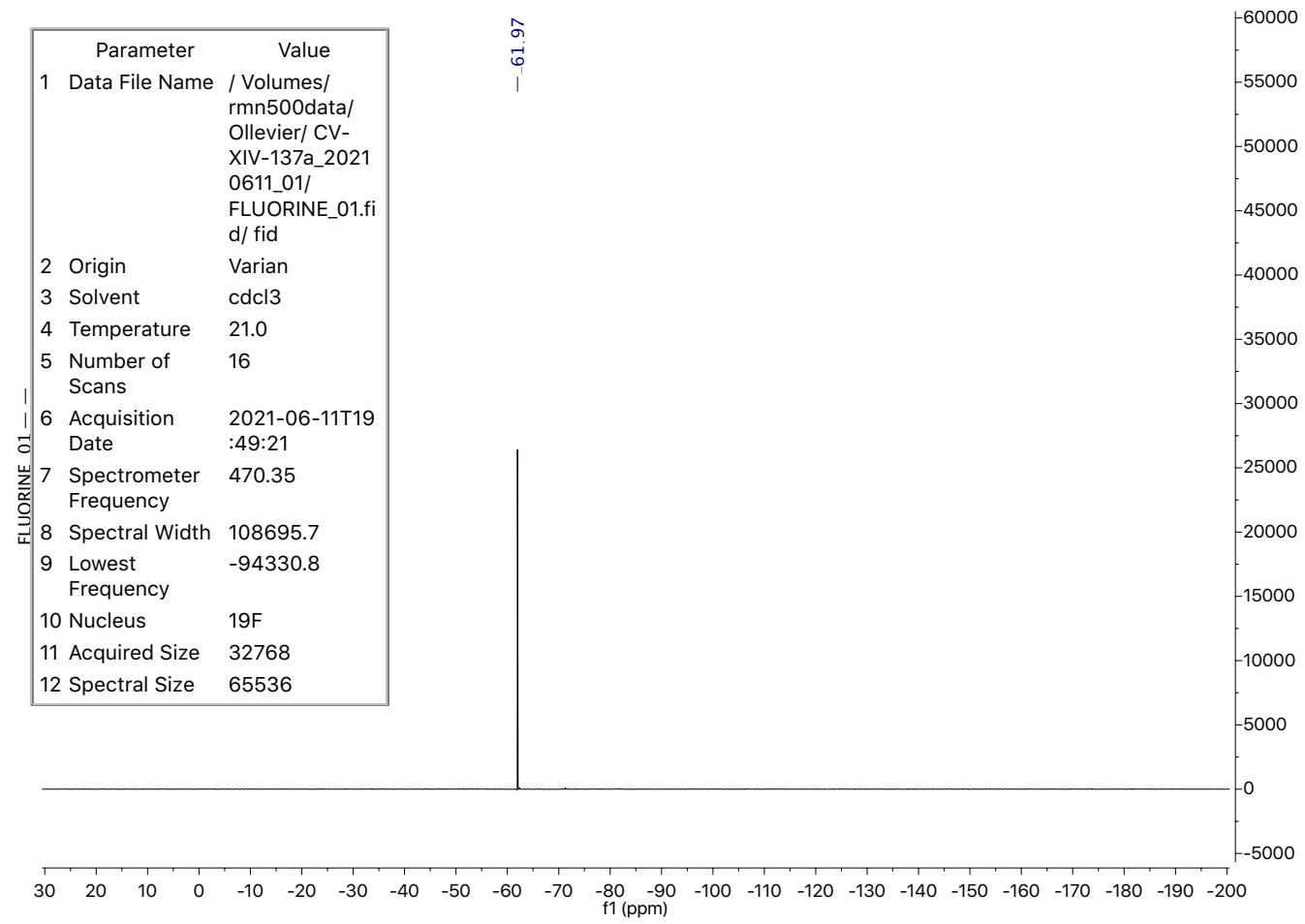




\section{B: $365 \mathrm{~nm}$}

\section{${ }^{1} \mathrm{H}$ NMR spectra of the mixture}

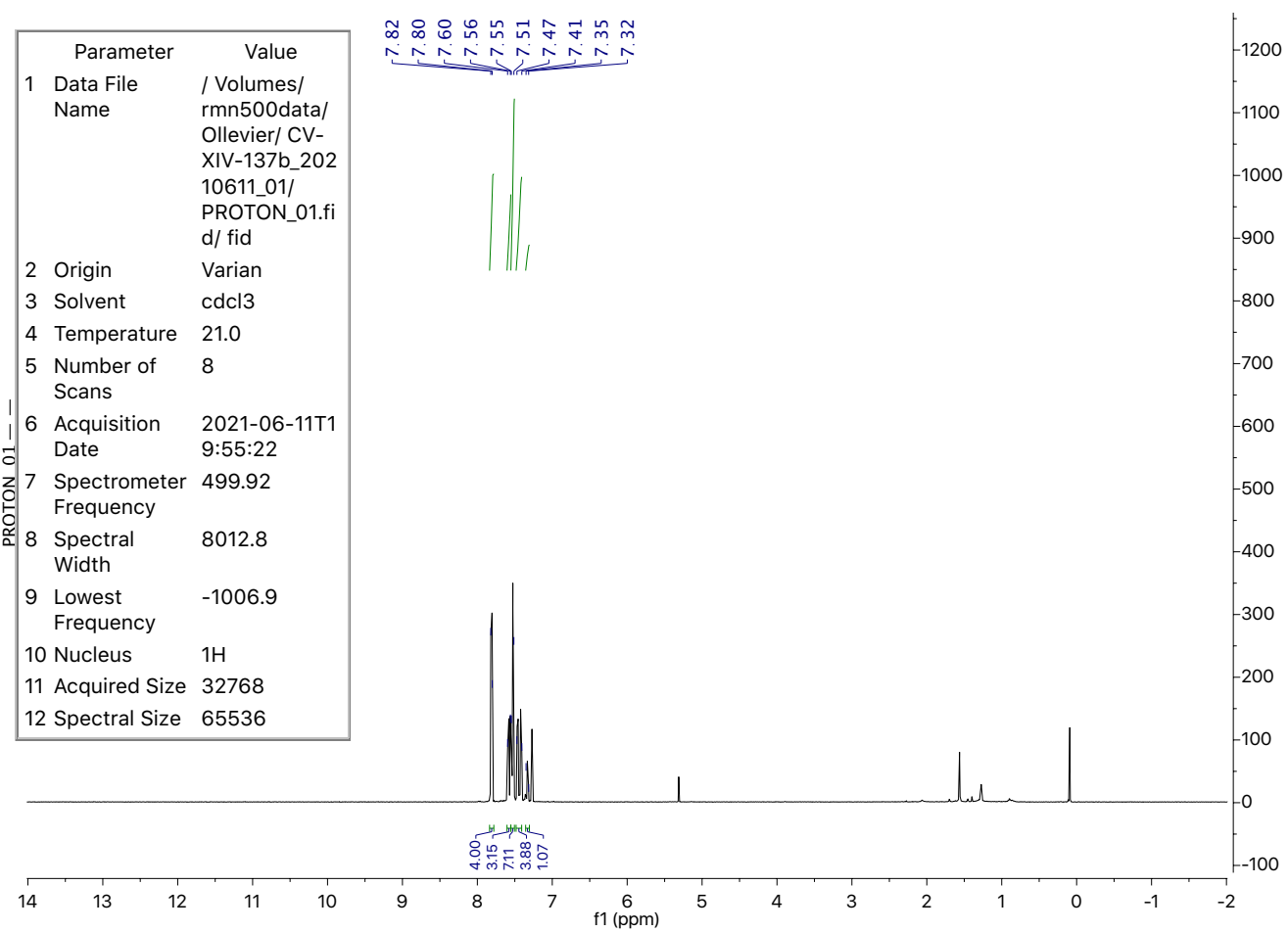

${ }^{19} \mathrm{~F}$ NMR spectra of the mixture

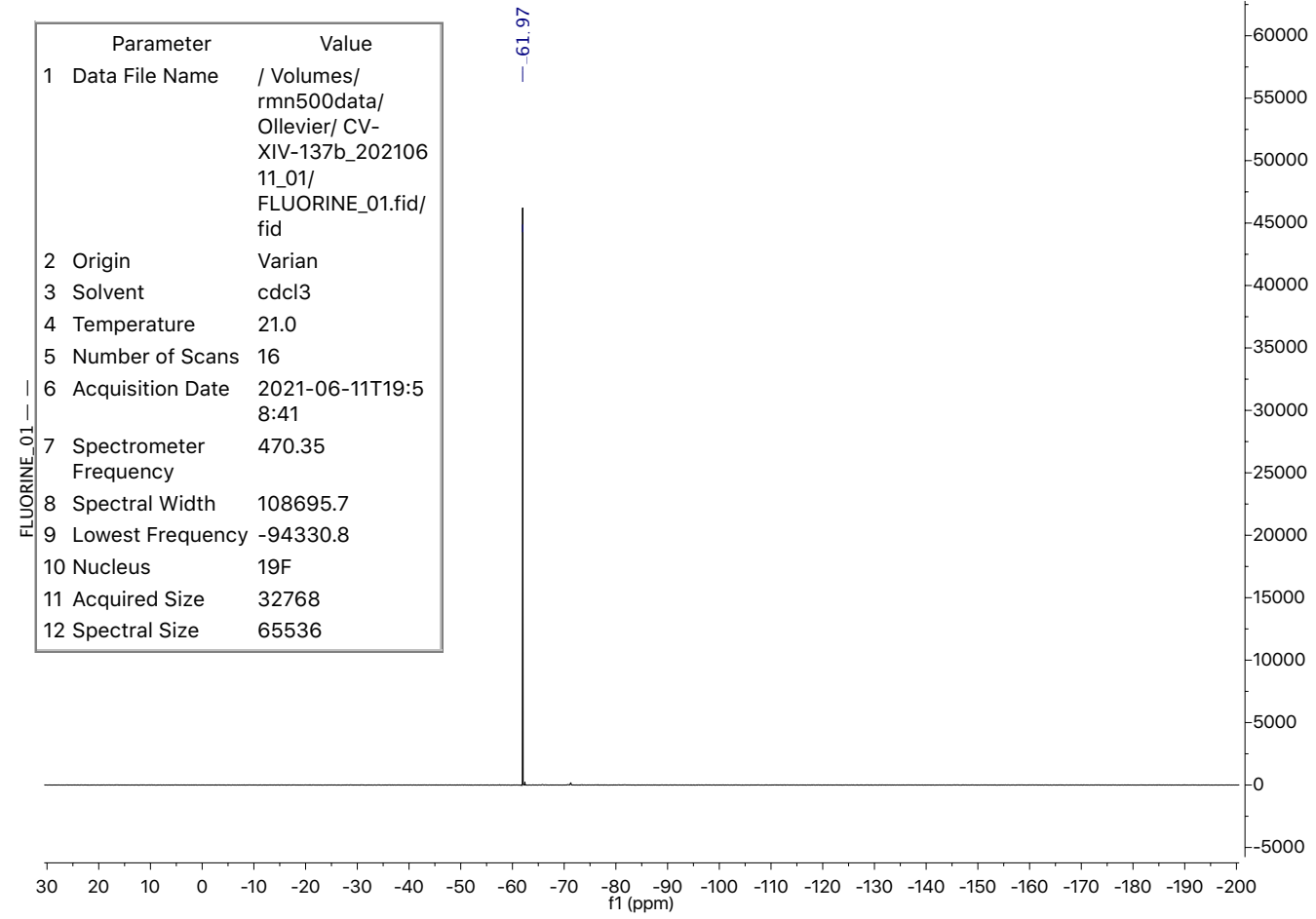




\section{References}

1. Manzi, L.; Barrow, A. S.; Scott, D.; Layfield, R.; Wright, T. G.; Moses, J. E.; Oldham, N. J., Carbene footprinting accurately maps binding sites in protein-ligand and protein-protein interactions. Nat. Commun. 2016, 7, 13288.

2. Richardson, S. K.; Ife, R. J., Swern oxidation of diaziridines to diazirines. J. Chem. Soc. Perkin Trans. 1989, 11721174.

3. Wang, L.; Yoshida, T.; Muto, Y.; Murai, Y.; Tachrim, Z. P.; Ishida, A.; Nakagawa, S.; Sakihama, Y.; Hashidoko, Y.; Masuda, K.; Hatanaka, Y.; Hashimoto, M., Synthesis of Diazirine-Based Photoreactive Saccharin Derivatives for the Photoaffinity Labeling of Gustatory Receptors. Eur. J. Org. Chem. 2015, 3129-3134.

4. Bender, T.; Huss, M.; Wieczorek, H.; Grond, S.; von Zezschwitz, P., Convenient Synthesis of a [114C]Diazirinylbenzoic Acid as a Photoaffinity Label for Binding Studies of V-ATPase Inhibitors. Eur. J. Org. Chem. 2007, 3870-3878.

5. Kogon, A. A.; Bochkariov, D. E.; Baskunov, B. P.; Cheprakov, A. V., 2,3-Dihydroxy-3-\{3-[3(trifluoromethyl)diazirin-3-yl]phenyl\}propionic Acid. A Cleavable Carbene-Generating Reagent Used for Photocrosslinking. Liebigs Ann. Chem. 1992, 1992, 879-881.

6. Lee, K.; Ban, H. S.; Naik, R.; Hong, Y. S.; Son, S.; Kim, B.-K.; Xia, Y.; Song, K. B.; Lee, H.-S.; Won, M., Identification of Malate Dehydrogenase 2 as a Target Protein of the HIF-1 Inhibitor LW6 using Chemical Probes. Angew. Chem., Int. Ed. 2013, 52, 10286-10289.

7. Protasova, I.; Bulat, B.; Jung, N.; Bräse, S., Synthesis of Diaziridines and Diazirines via Resin-Bound Sulfonyl Oximes. Org. Lett. 2017, 19, 34-37.

8. Hatanaka, Y.; Hashimoto, M.; Kurihara, H.; Nakayama, H.; Kanaoka, Y., A Novel Family of Aromatic Diazirines for Photoaffinity Labeling. J. Org. Chem. 1994, 59, 383-387.

9. Protasova, I.; Bulat, B.; Jung, N.; Brase, S., Synthesis of Diaziridines and Diazirines via Resin-Bound Sulfonyl Oximes. Org. Lett. 2017, 19, 34-37.

10. Wang, L.; Murai, Y.; Yoshida, T.; Ishida, A.; Masuda, K.; Sakihama, Y.; Hashidoko, Y.; Hatanaka, Y.; Hashimoto, M., Alternative one-pot synthesis of (trifluoromethyl)phenyldiazirines from tosyloxime derivatives: application for new synthesis of optically pure diazirinylphenylalanines for photoaffinity labeling. Org. Lett. 2015, 17, 616-619.

11. Barroso, R.; Jimenez, A.; Perez-Aguilar, M. C.; Cabal, M. P.; Valdes, C., Synthesis of 1,3-diaryl-3trifluoromethylcyclopropenes by transition-metal-free reaction of 2,2,2-trifluoroacetophenone tosylhydrazones with alkynes: the effect of the trifluoromethyl group. Chem. Commun. 2016, 52, 3677-3680. 
8. NMR Spectra

\section{1-([1,1'-Biphenyl]-4-yl)-2,2,2-trifluoroethan-1-one 0-tosyl oxime a1}

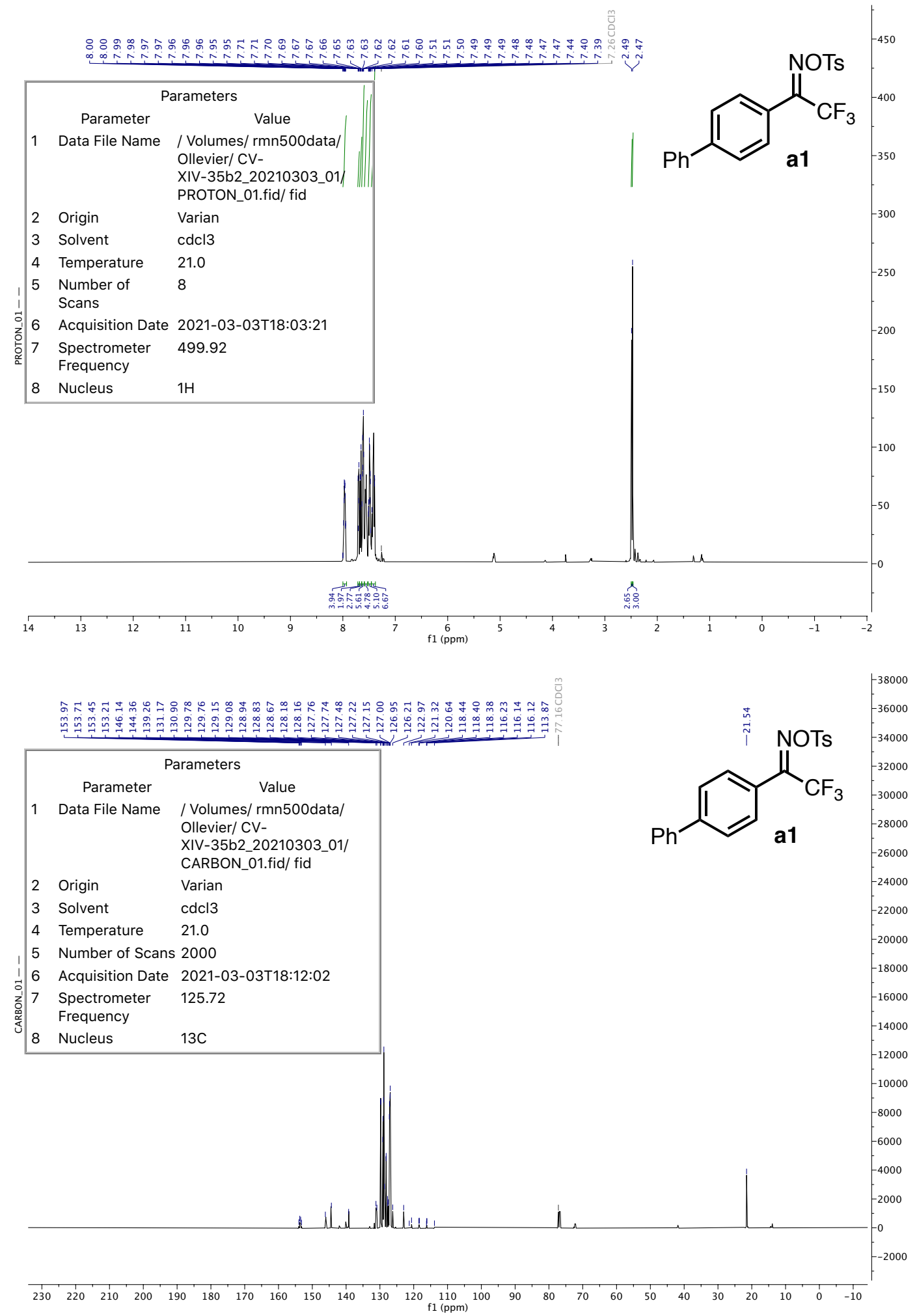




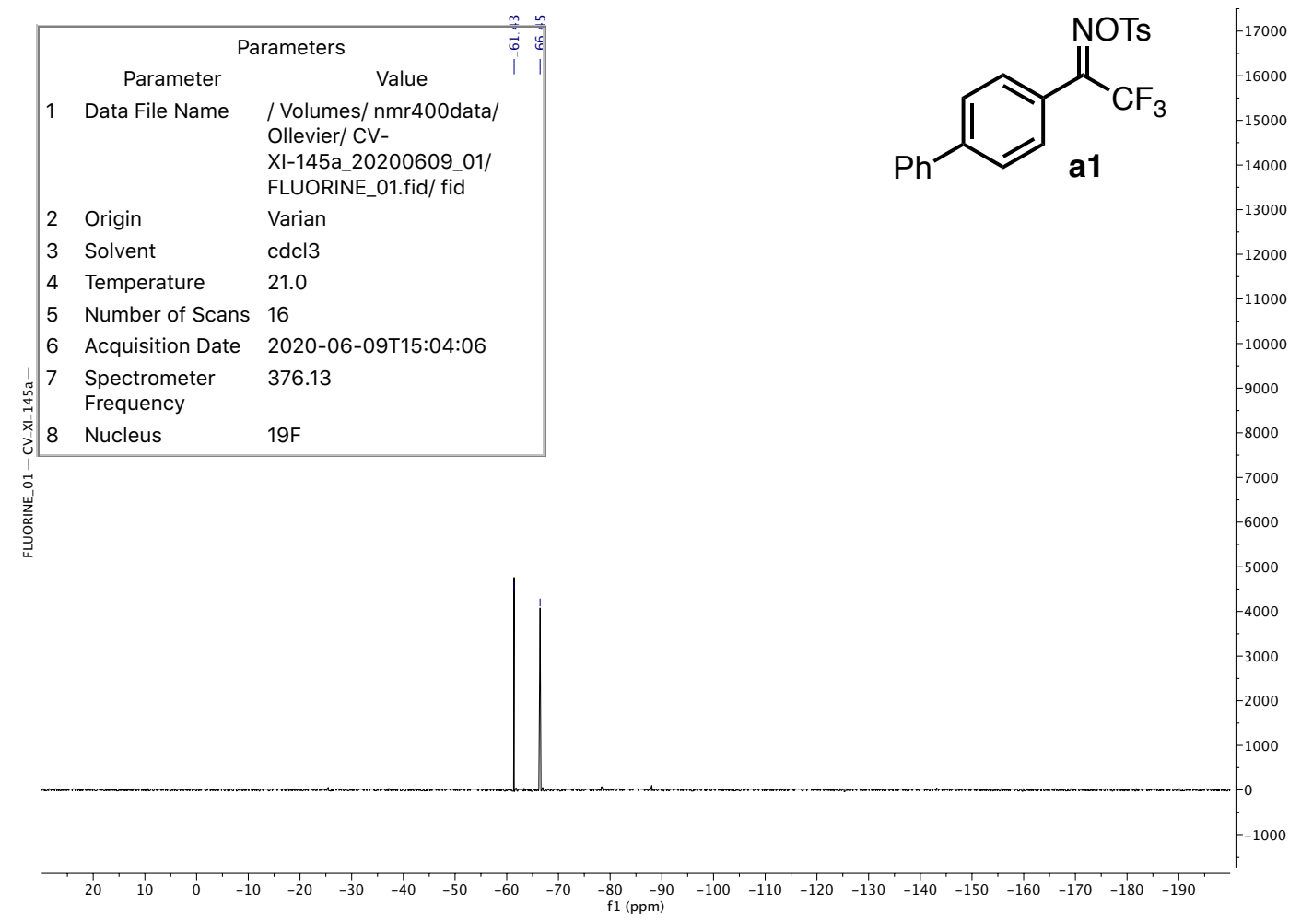

\section{2,2,2-Trifluoro-1-(naphthalen-2-yl)ethan-1-one 0-tosyl oxime b1}

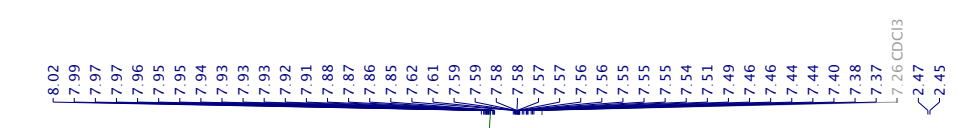

\begin{tabular}{|c|c|c|}
\hline & \multicolumn{2}{|c|}{ Parameters } \\
\hline & Parameter & Value \\
\hline 1 & Data File Name & $\begin{array}{l}\text { / Volumes/ rmn500data/ } \\
\text { Ollevier/ CV- } \\
\text { XI-151_20210211_01/ } \\
\text { PROTON_01.fid/fid }\end{array}$ \\
\hline 2 & Origin & Varian \\
\hline 3 & Solvent & cdcl3 \\
\hline 4 & Temperature & 22.0 \\
\hline 5 & Number of Scans & 8 \\
\hline 6 & Acquisition Date & 2021-02-11T18:08:06 \\
\hline 7 & $\begin{array}{l}\text { Spectrometer } \\
\text { Frequency }\end{array}$ & 499.92 \\
\hline 8 & Nucleus & $1 \mathrm{H}$ \\
\hline
\end{tabular}

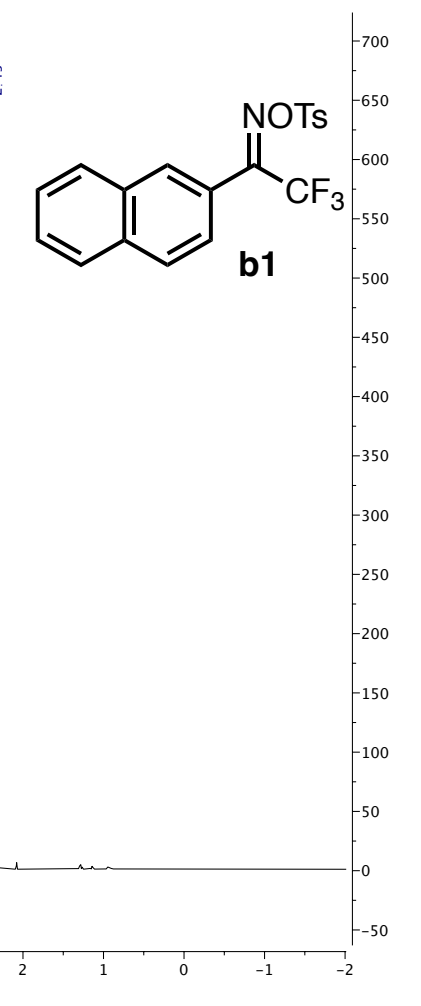



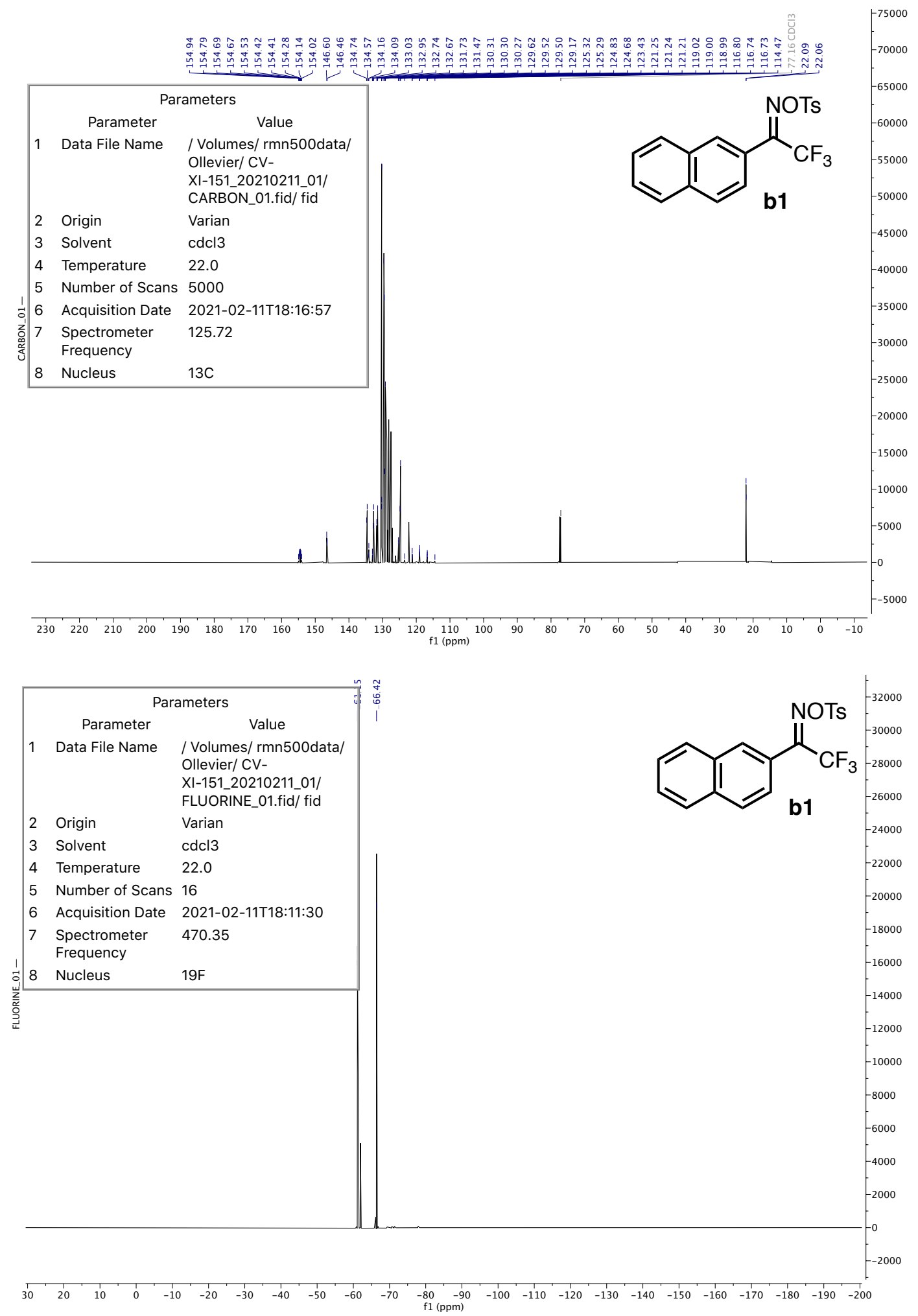
1-(4-(Benzyloxy)phenyl)-2,2,2-trifluoroethan-1-one 0-tosyl oxime c1

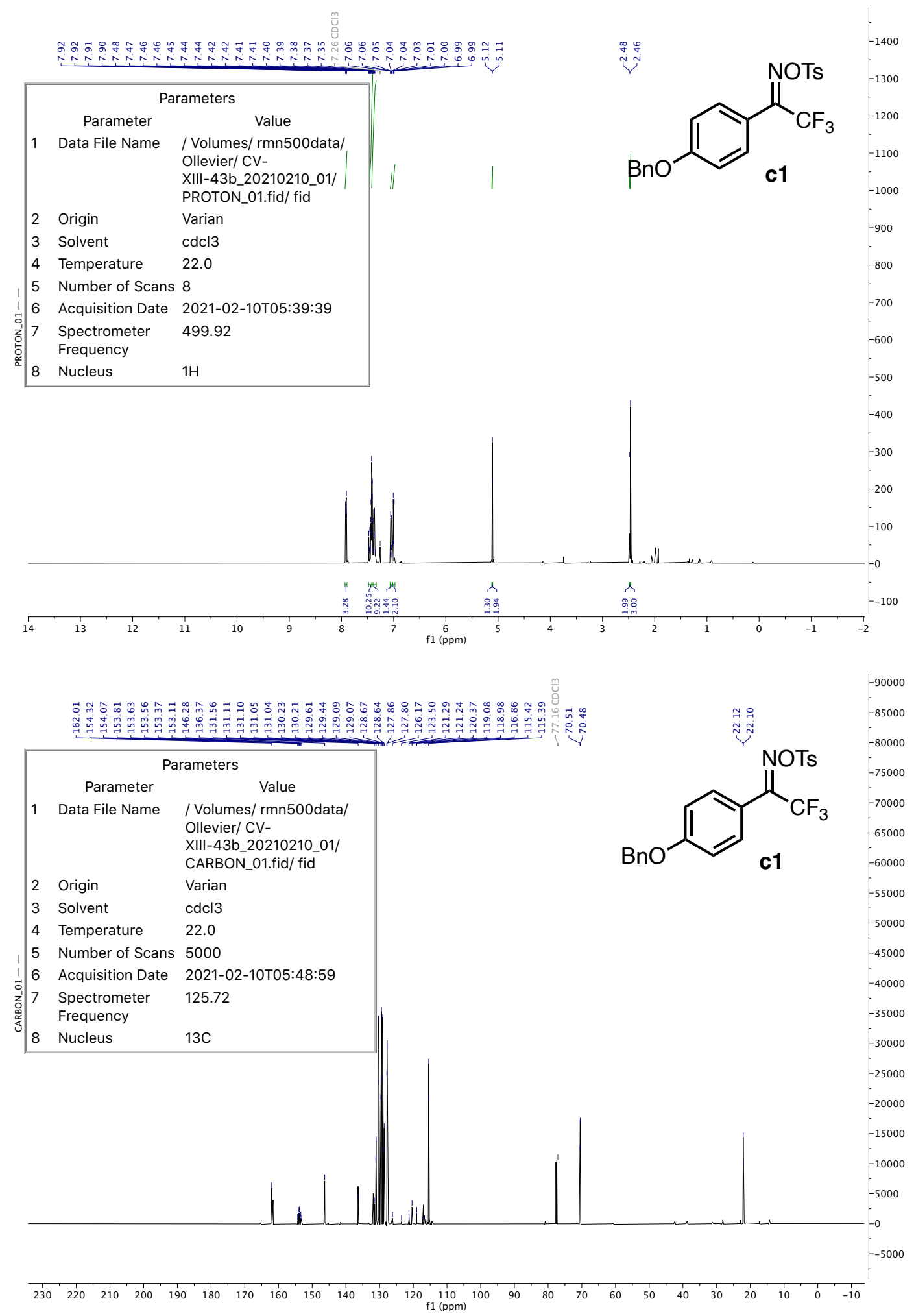




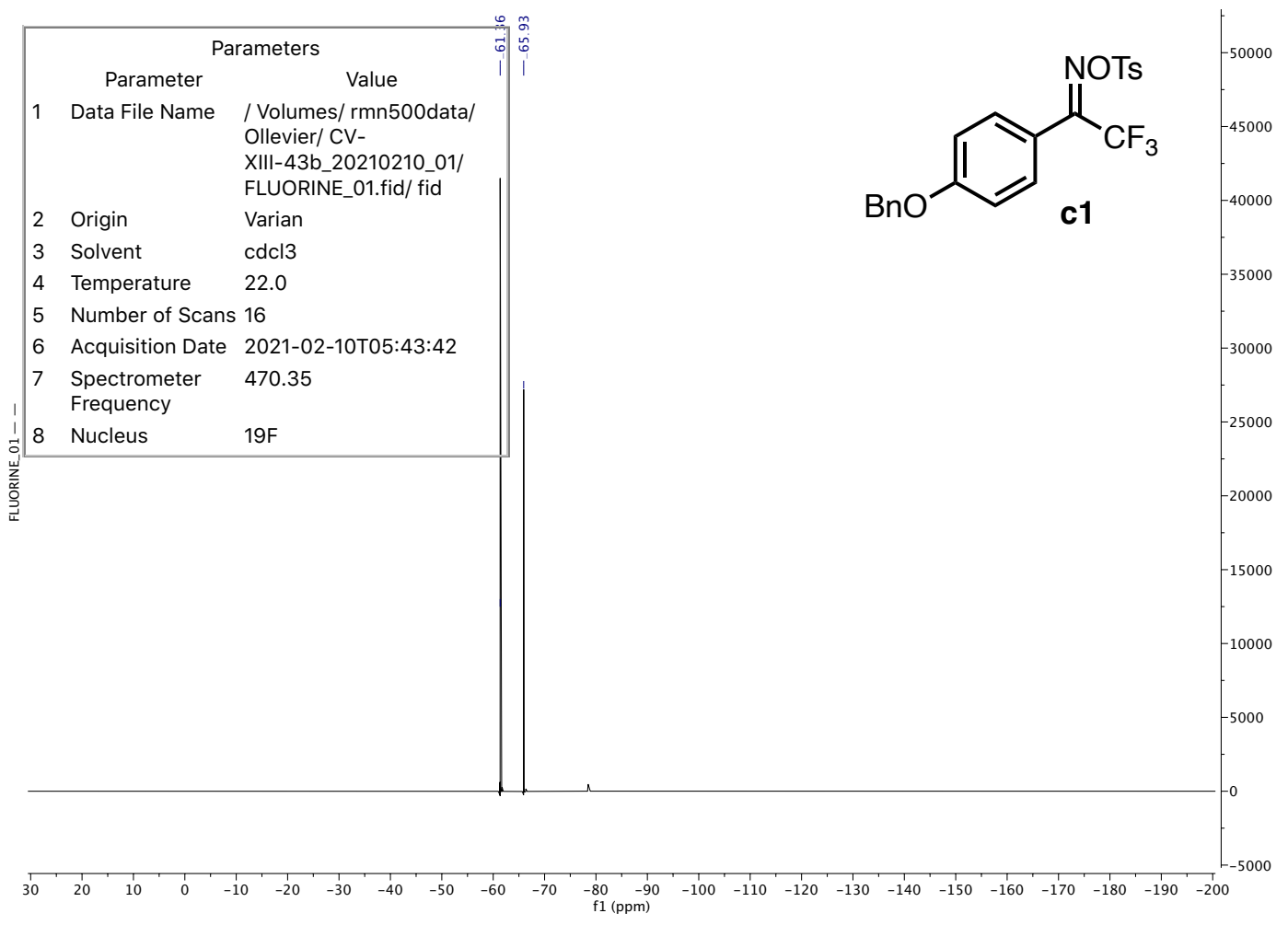

\section{1,1'-([1,1'-Biphenyl]-4,4'-diyl)bis(2,2,2-trifluoroethan-1-one) 0,0-ditosyl dioxime d1}

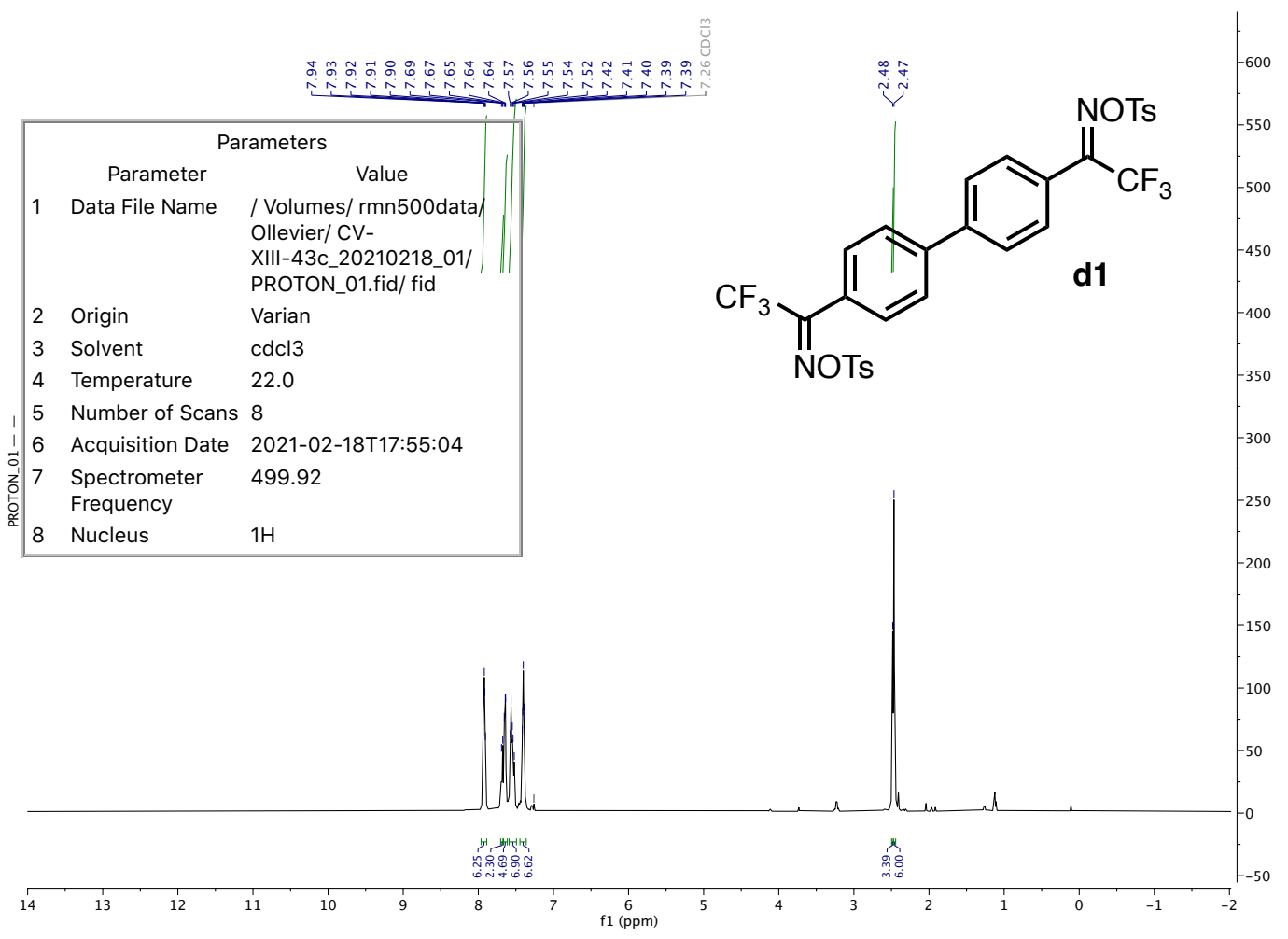




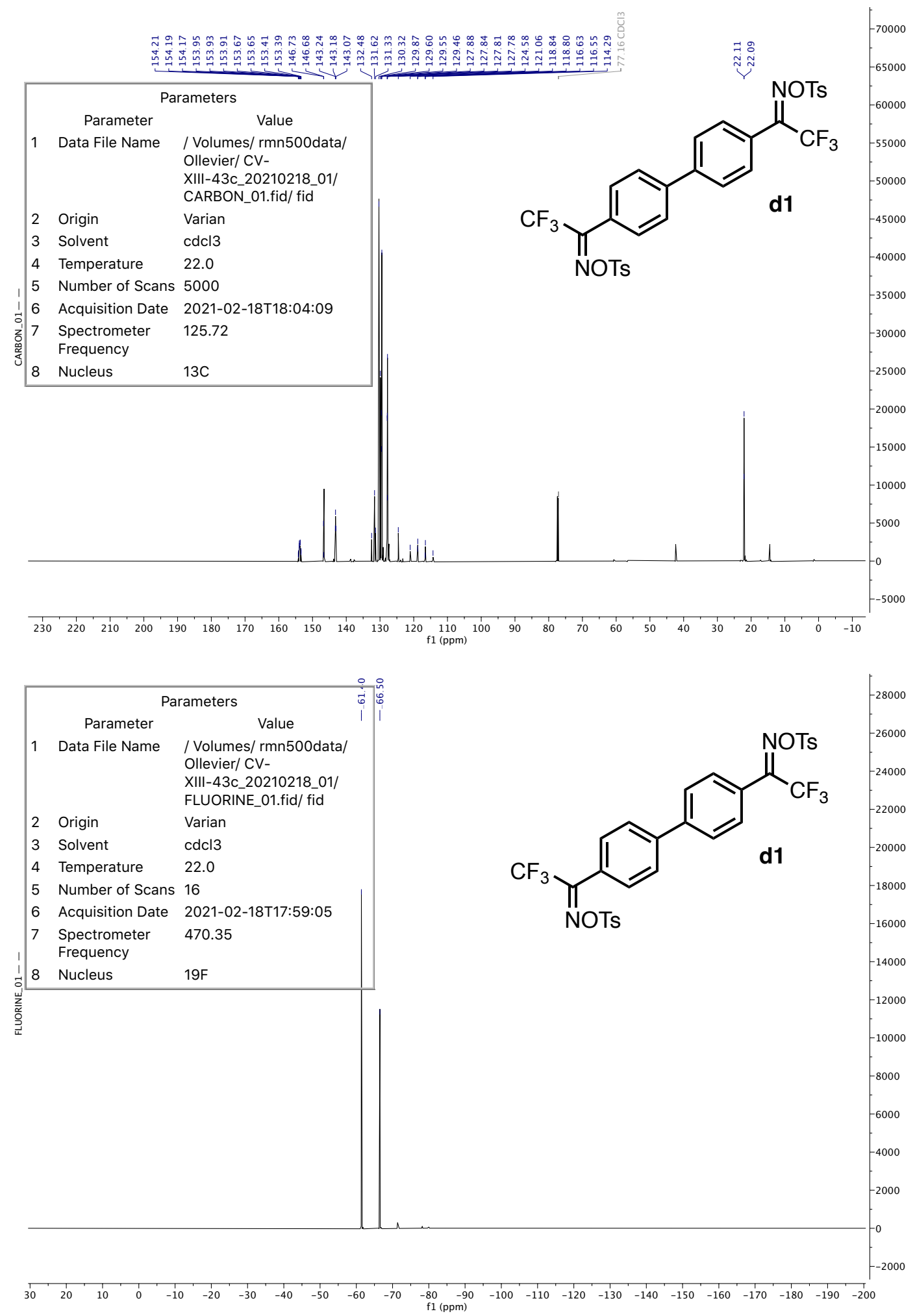




\section{2,2,2-Trifluoro-1-(p-tolyl)ethan-1-one 0-tosyl oxime e1}

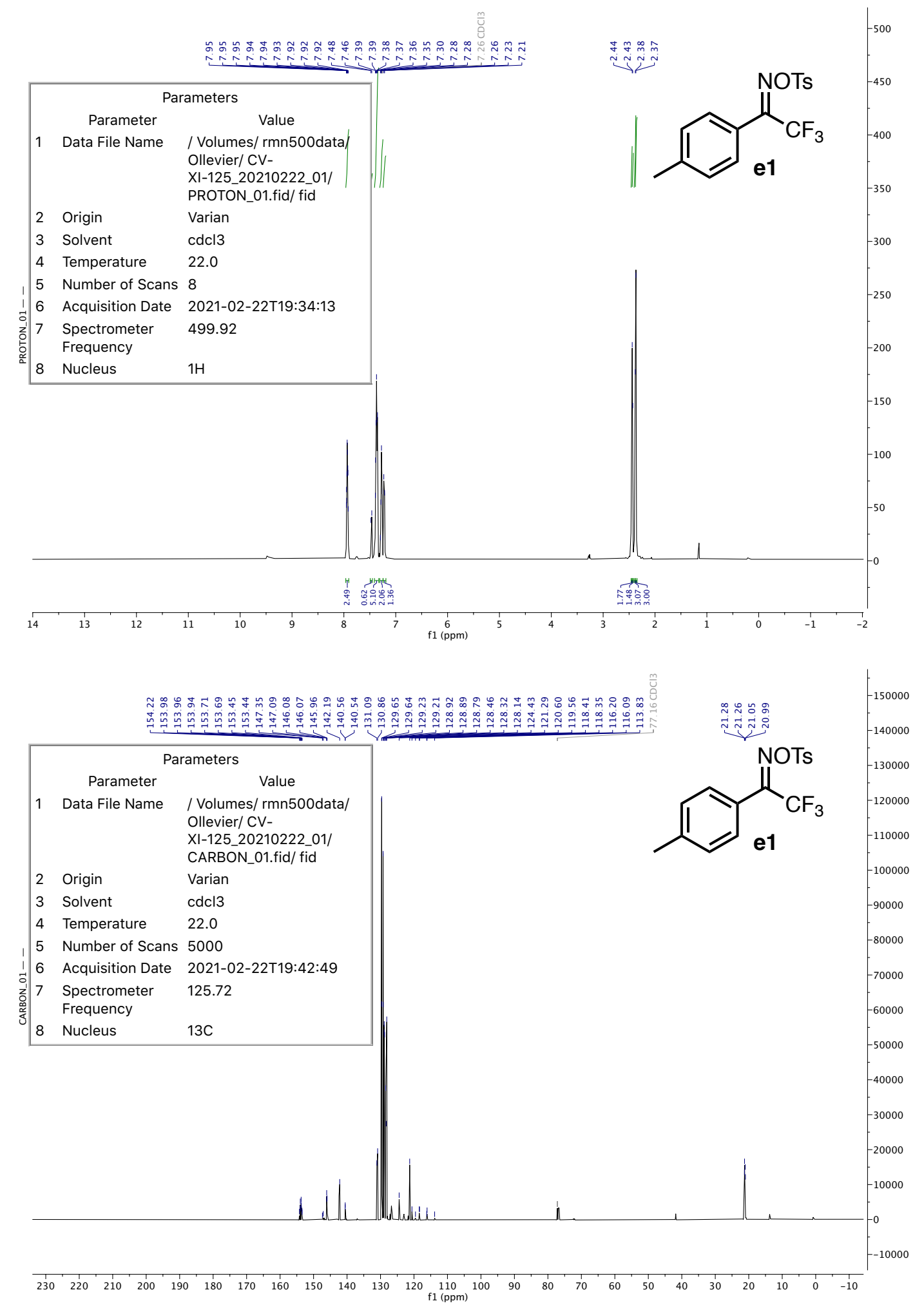




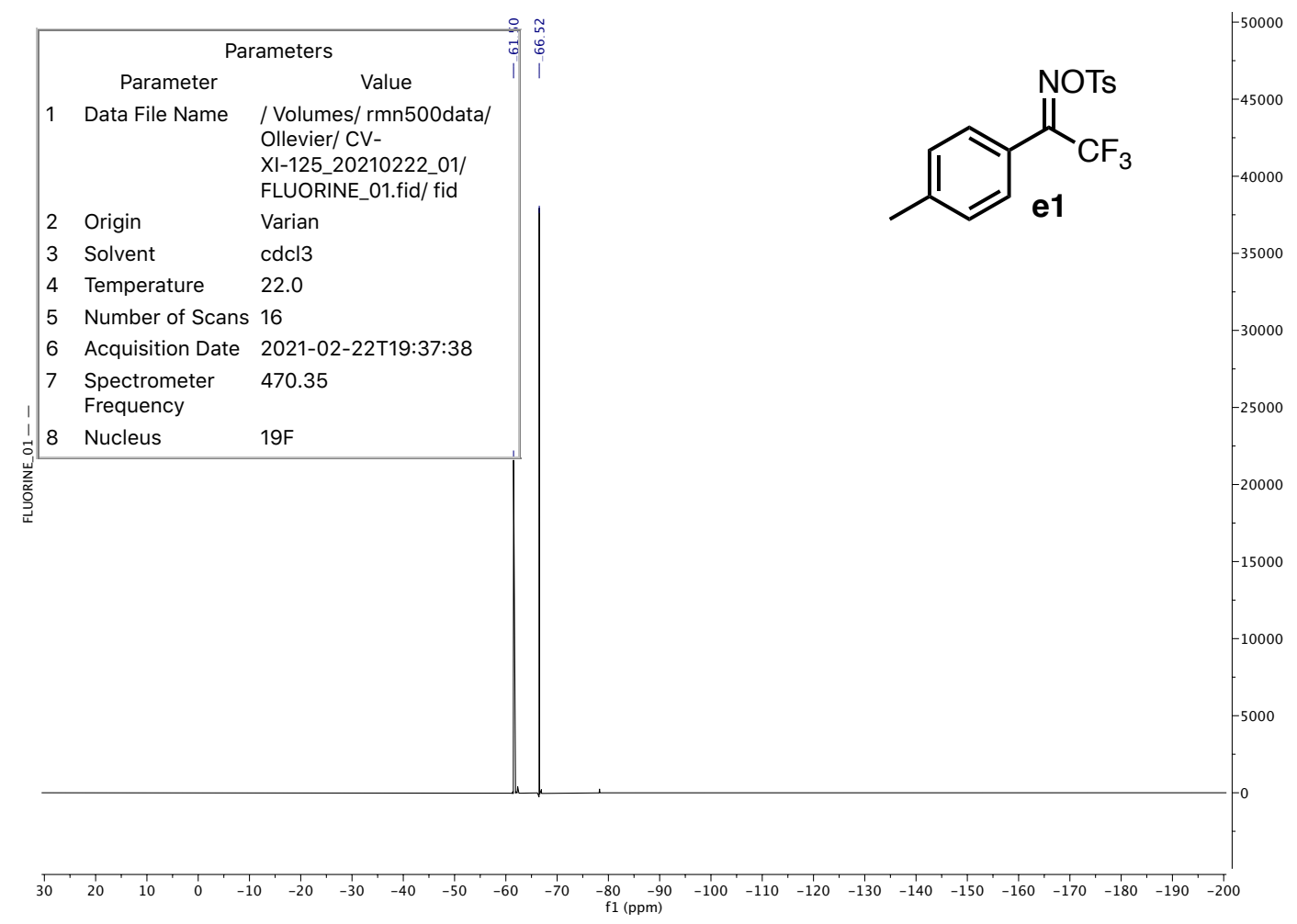

\section{1-(4-(Tert-butyl)phenyl)-2,2,2-trifluoroethan-1-one 0-tosyl oxime f1}

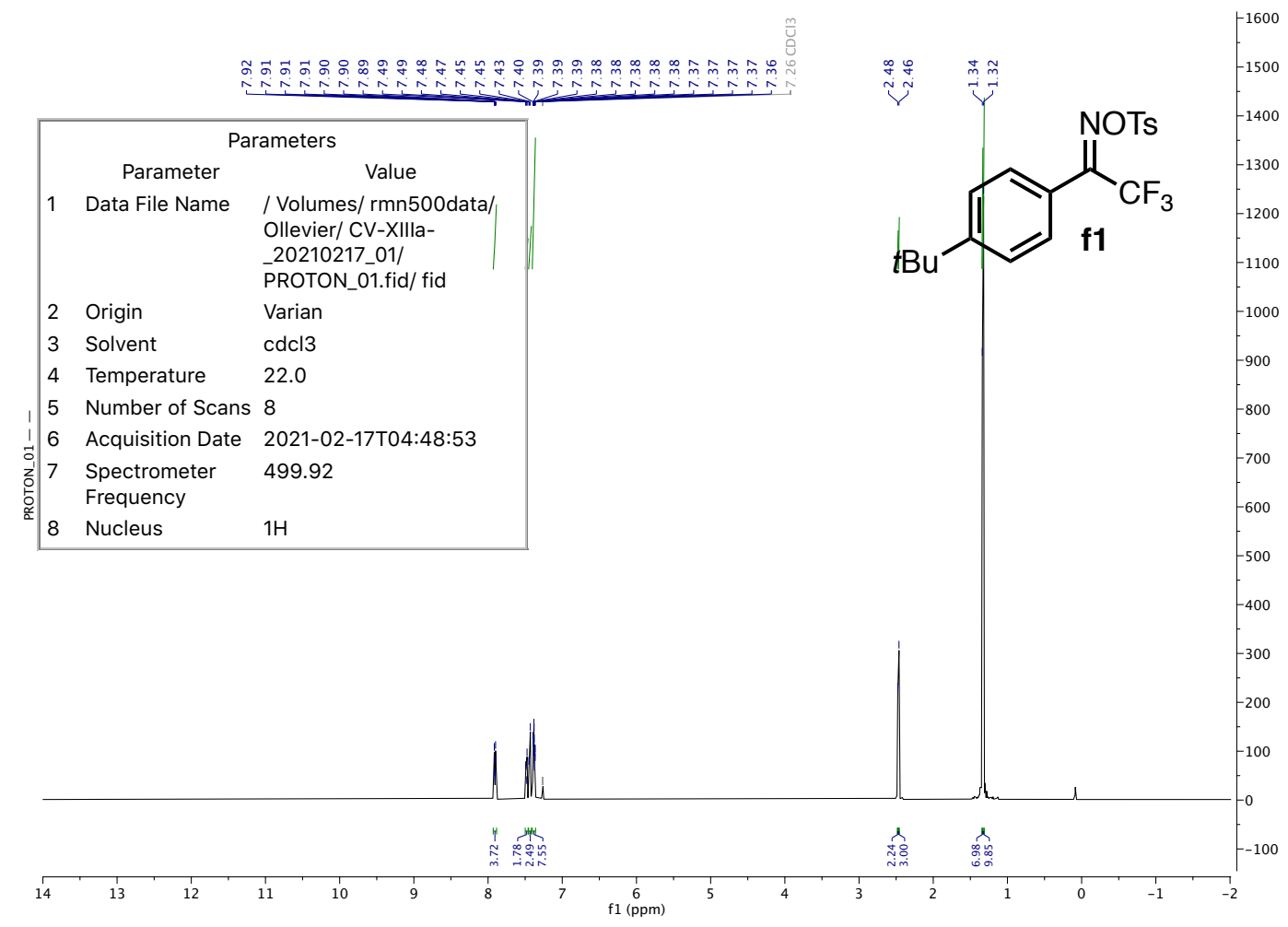



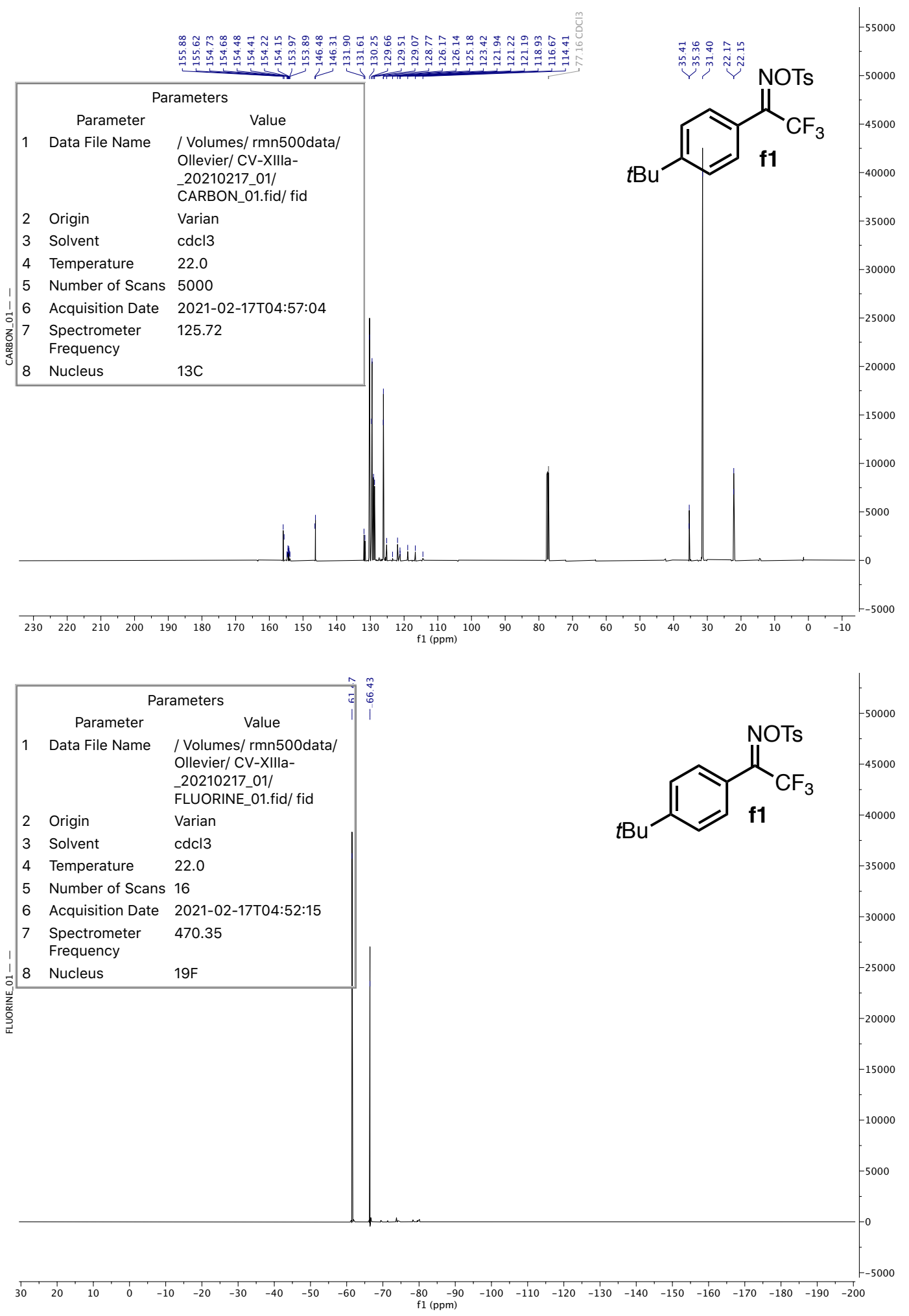


\section{2,2,2-Trifluoro-1-(4-methoxyphenyl)ethan-1-one 0-tosyl oxime g1}
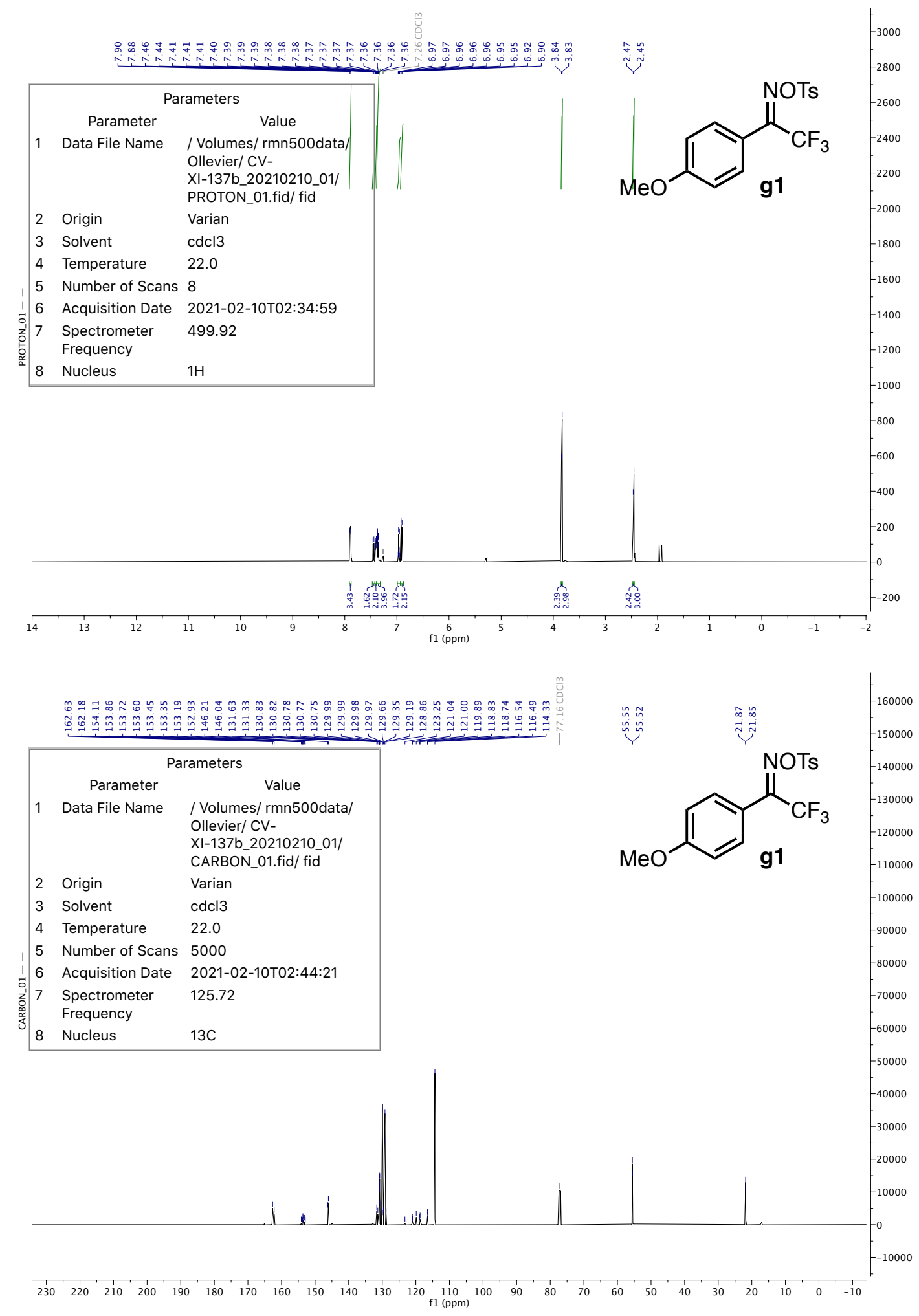


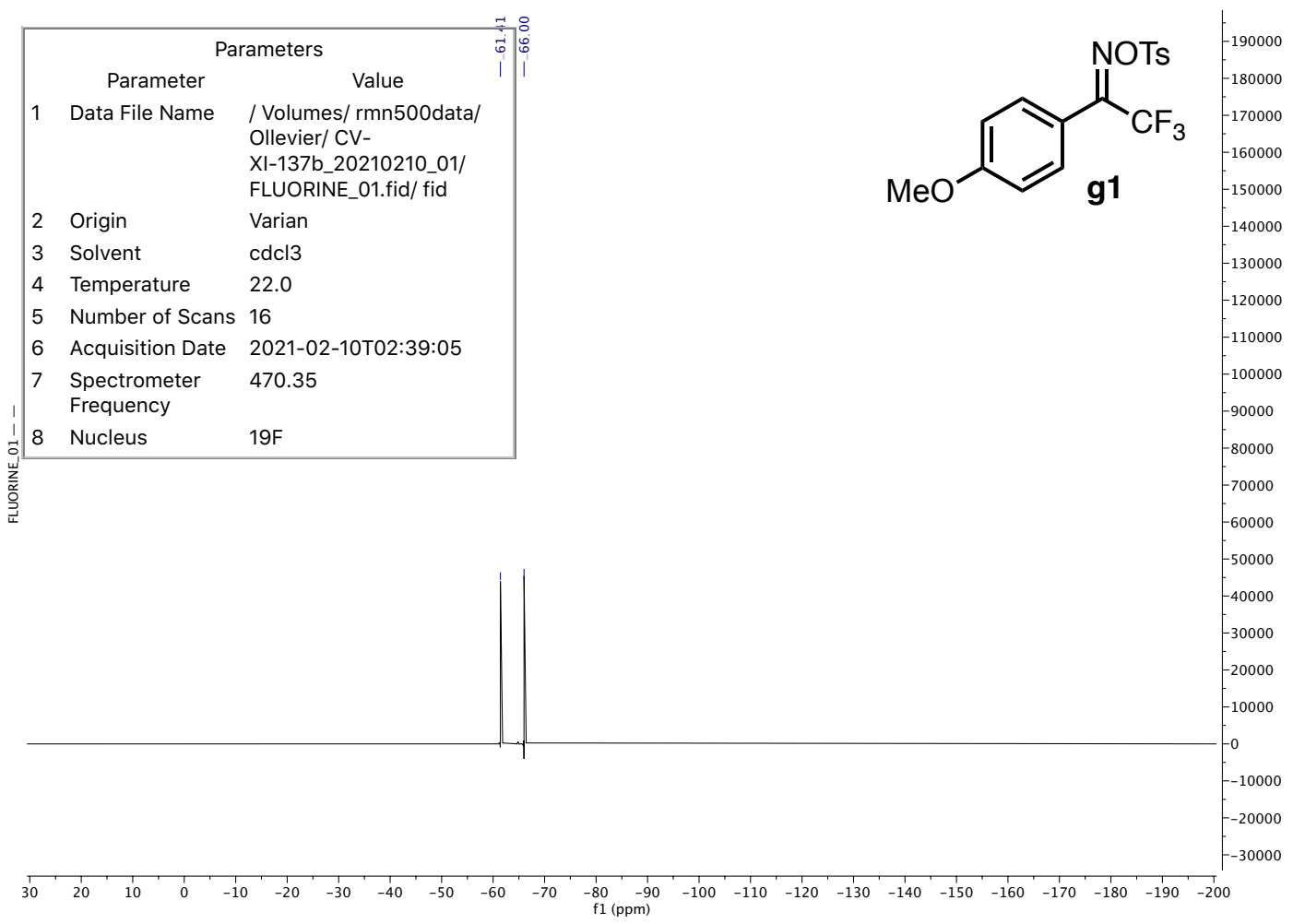

\section{2,2,2-Trifluoro-1-phenylethan-1-one 0-tosyl oxime h1}

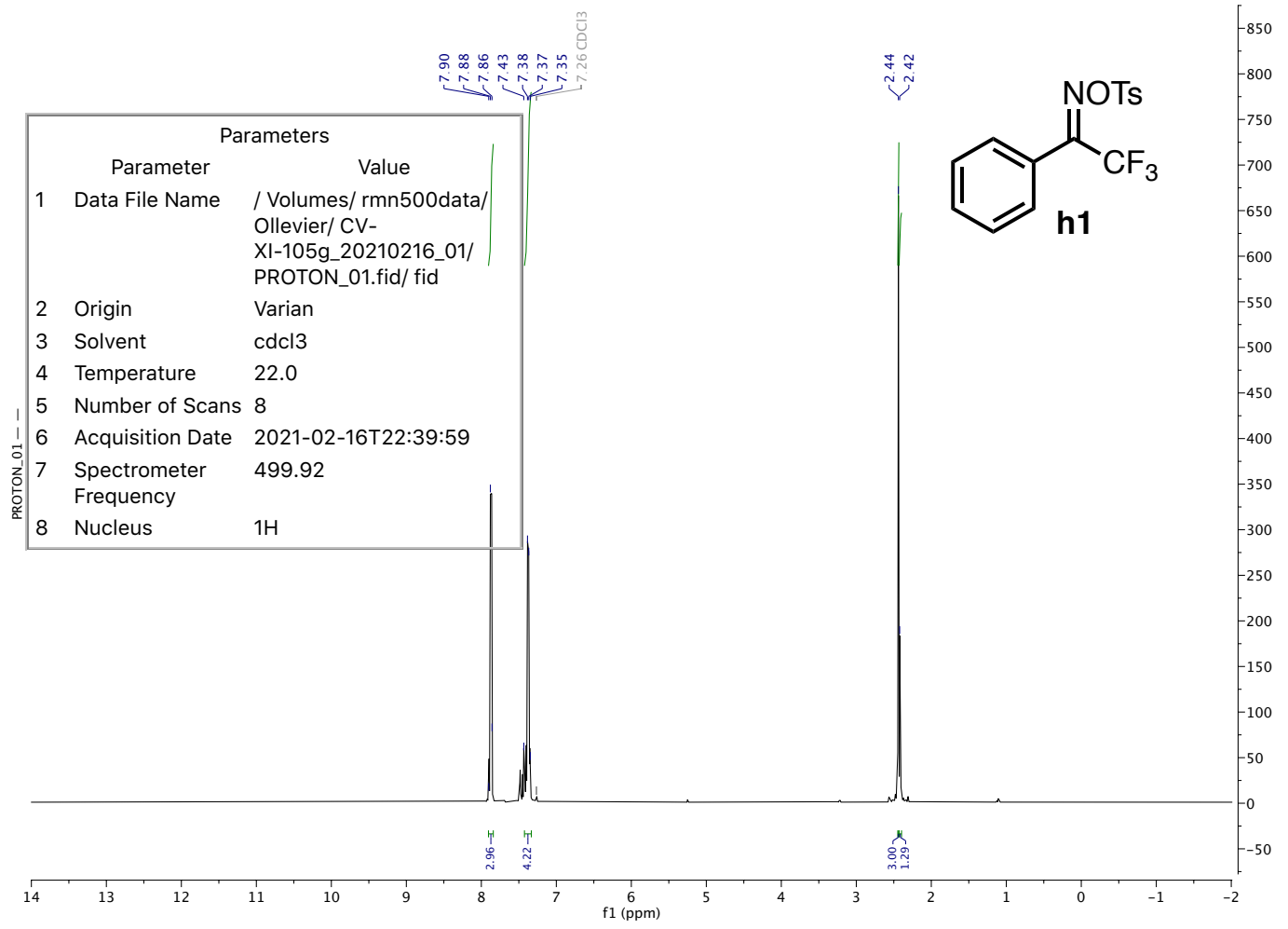



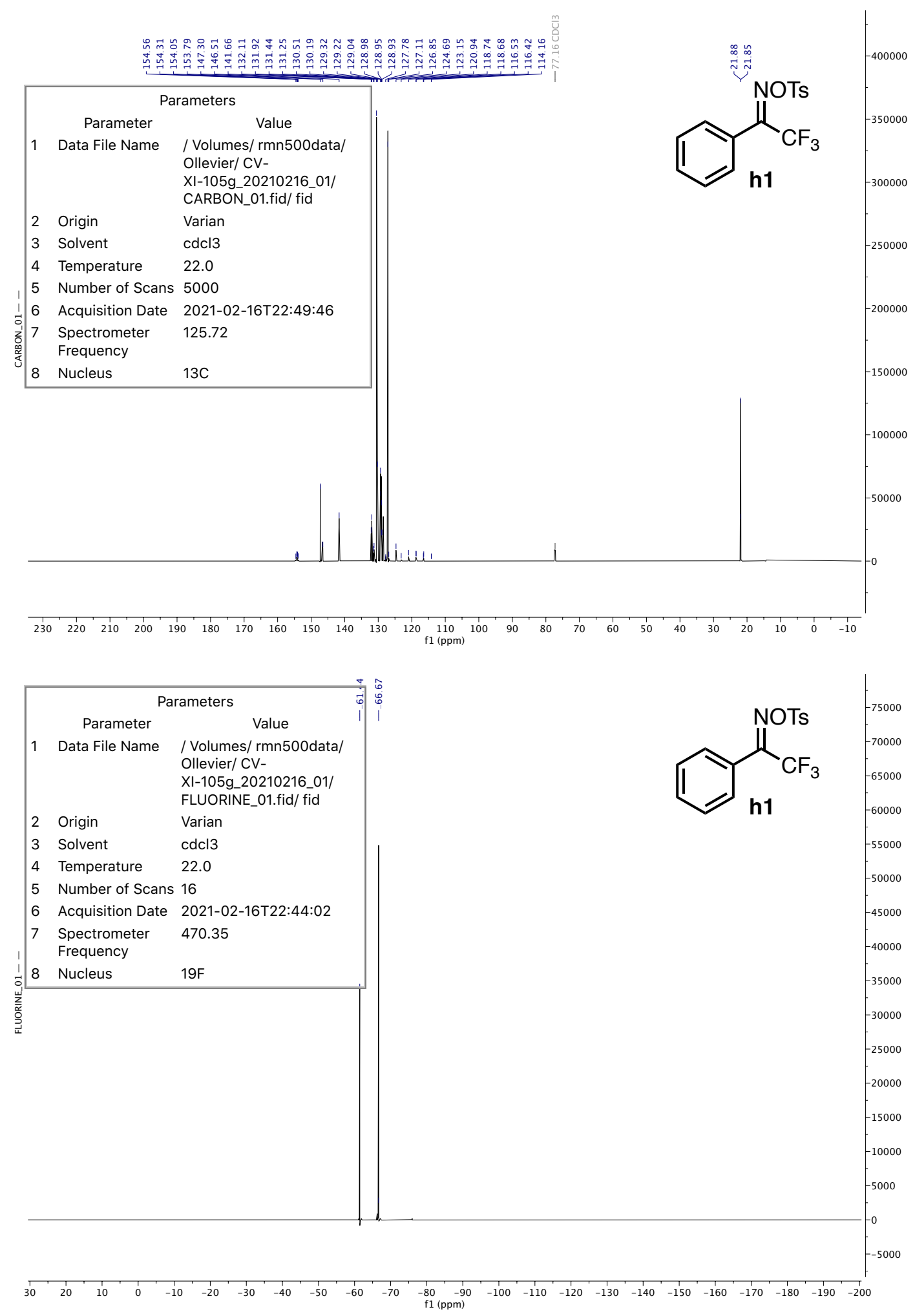


\section{2,2,2-Trifluoro-1-(4-fluorophenyl)ethan-1-one 0-tosyl oxime i1}

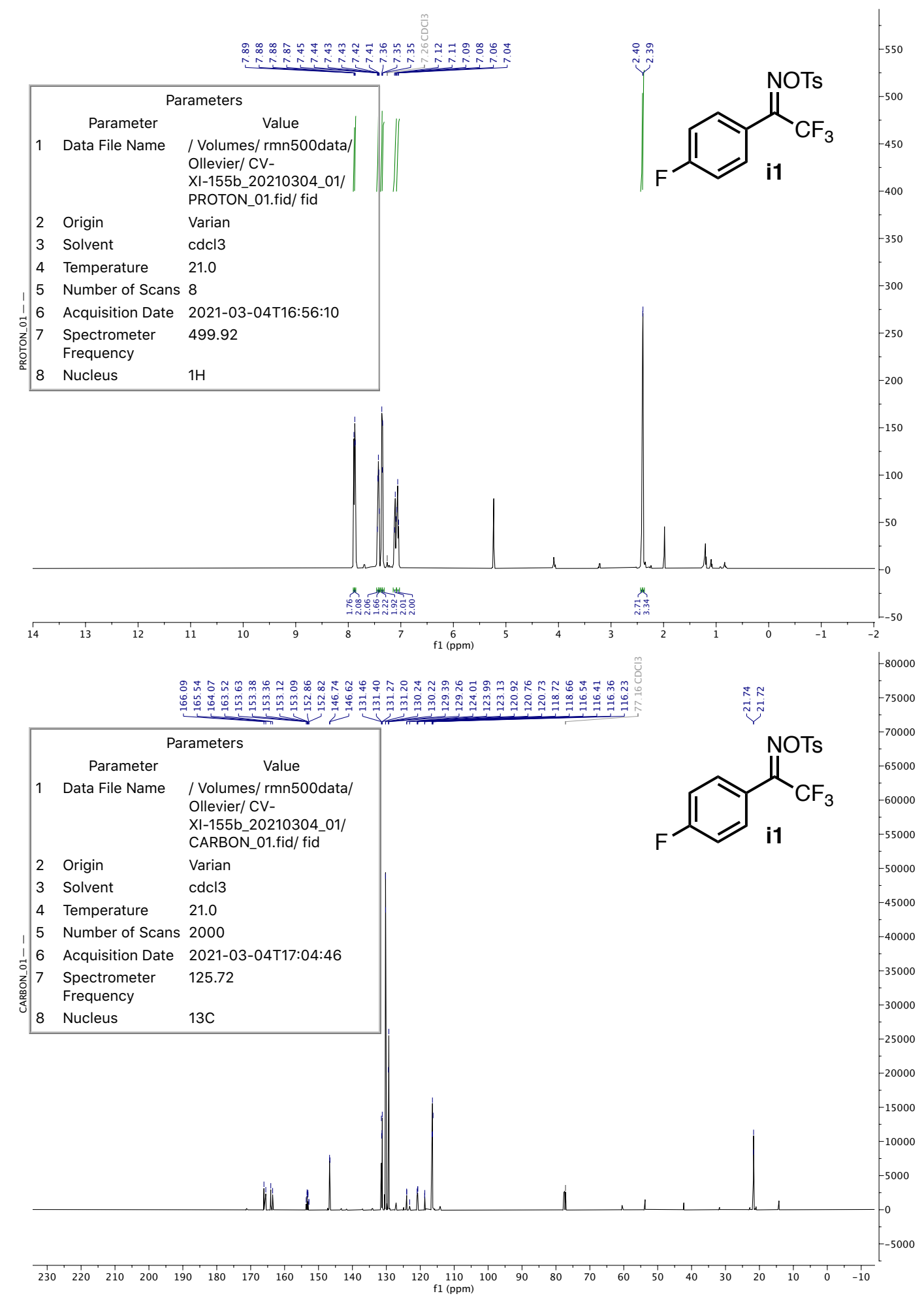




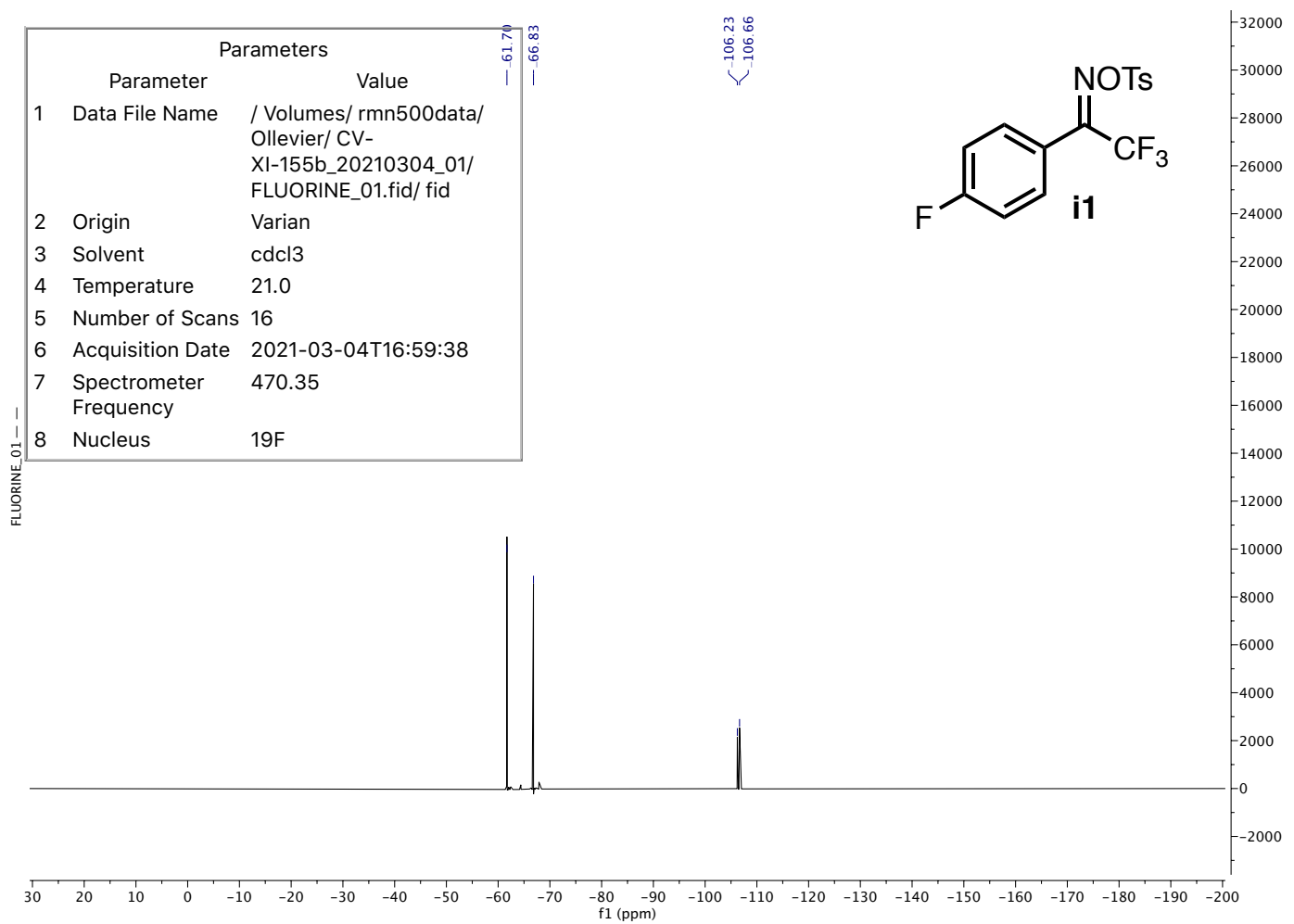

\section{1-(4-Bromophenyl)-2,2,2-trifluoroethan-1-one 0-tosyl oxime j1}

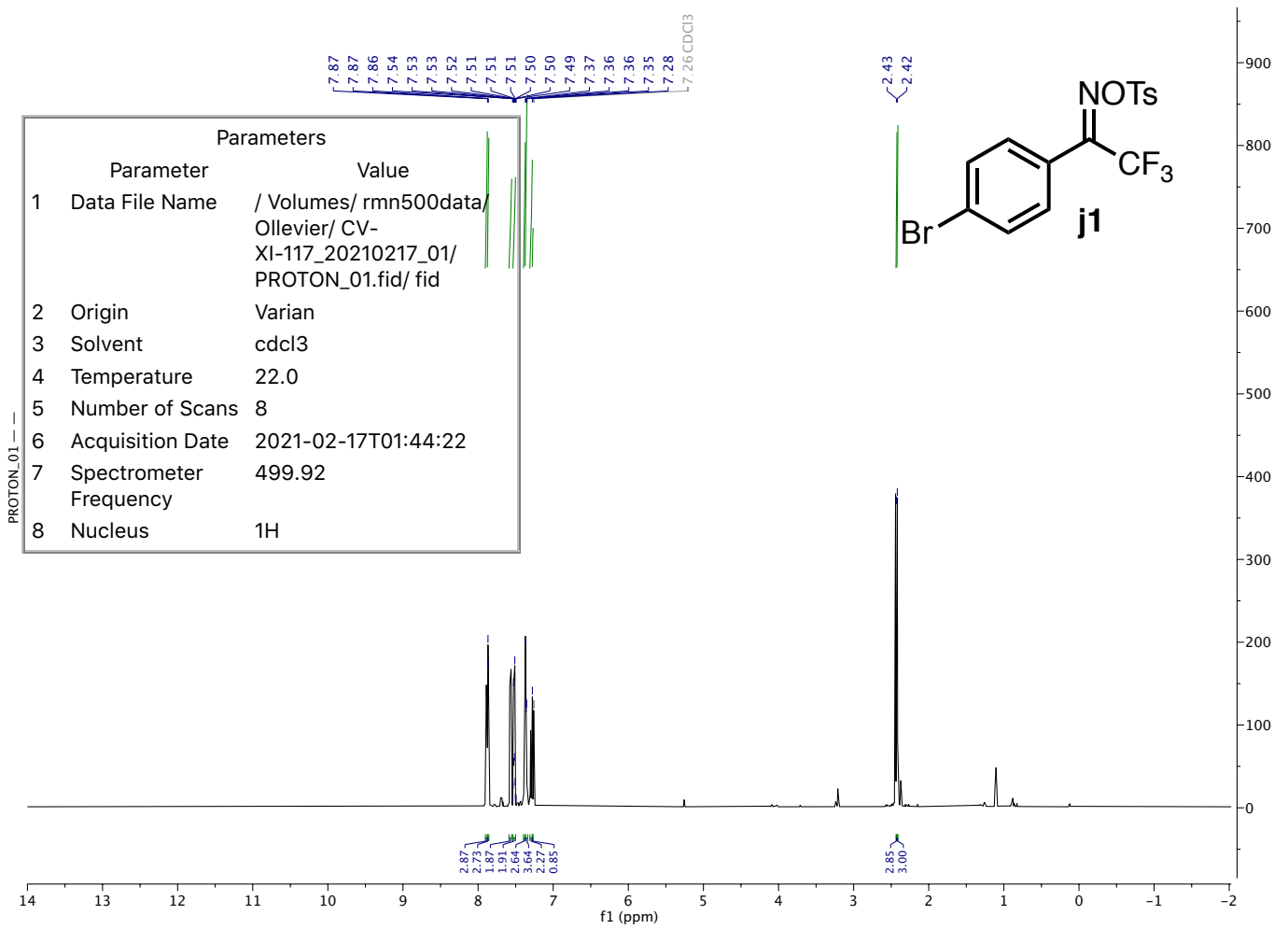




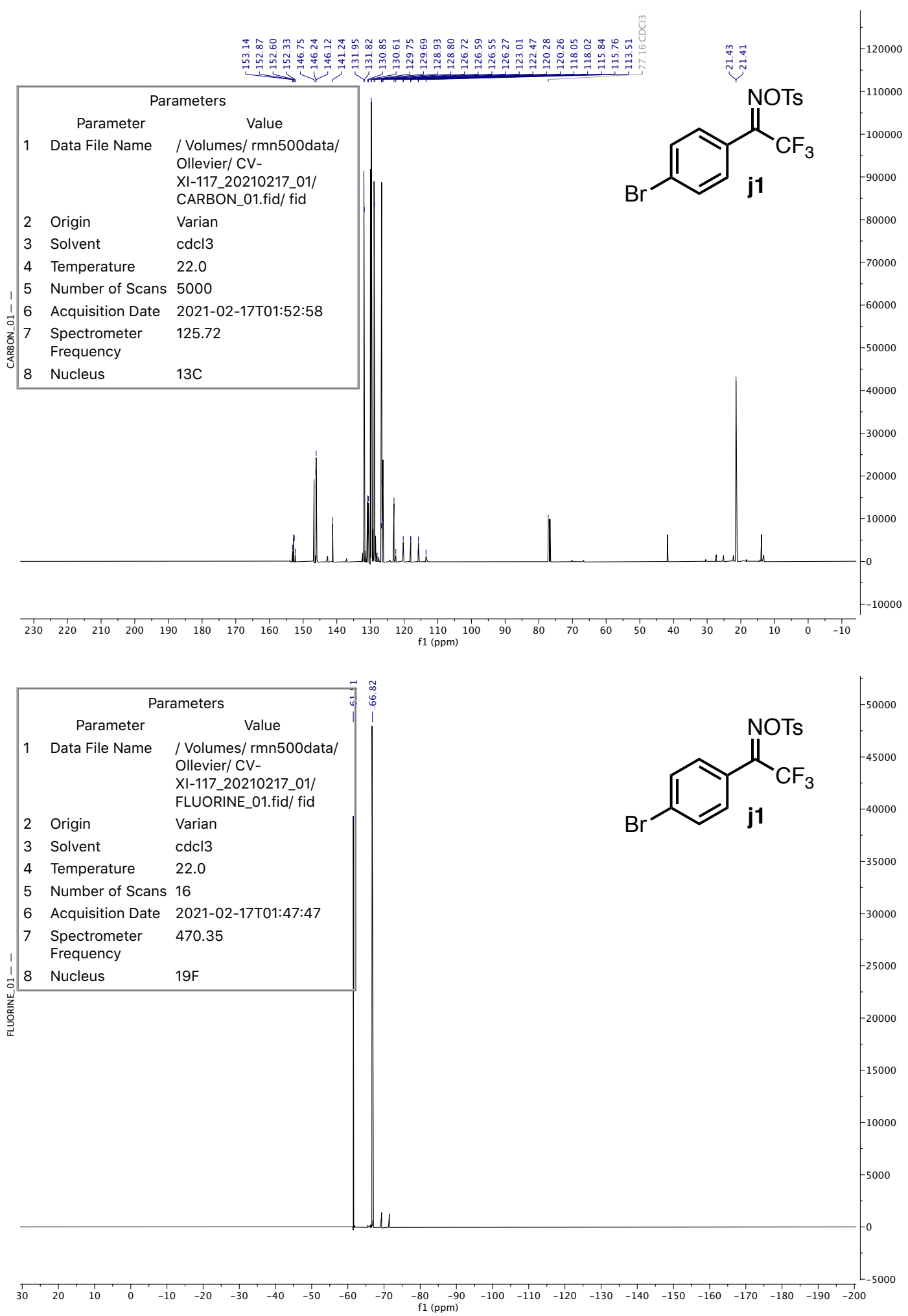




\section{1-(3-Bromophenyl)-2,2,2-trifluoroethan-1-one 0-tosyl oxime k1}
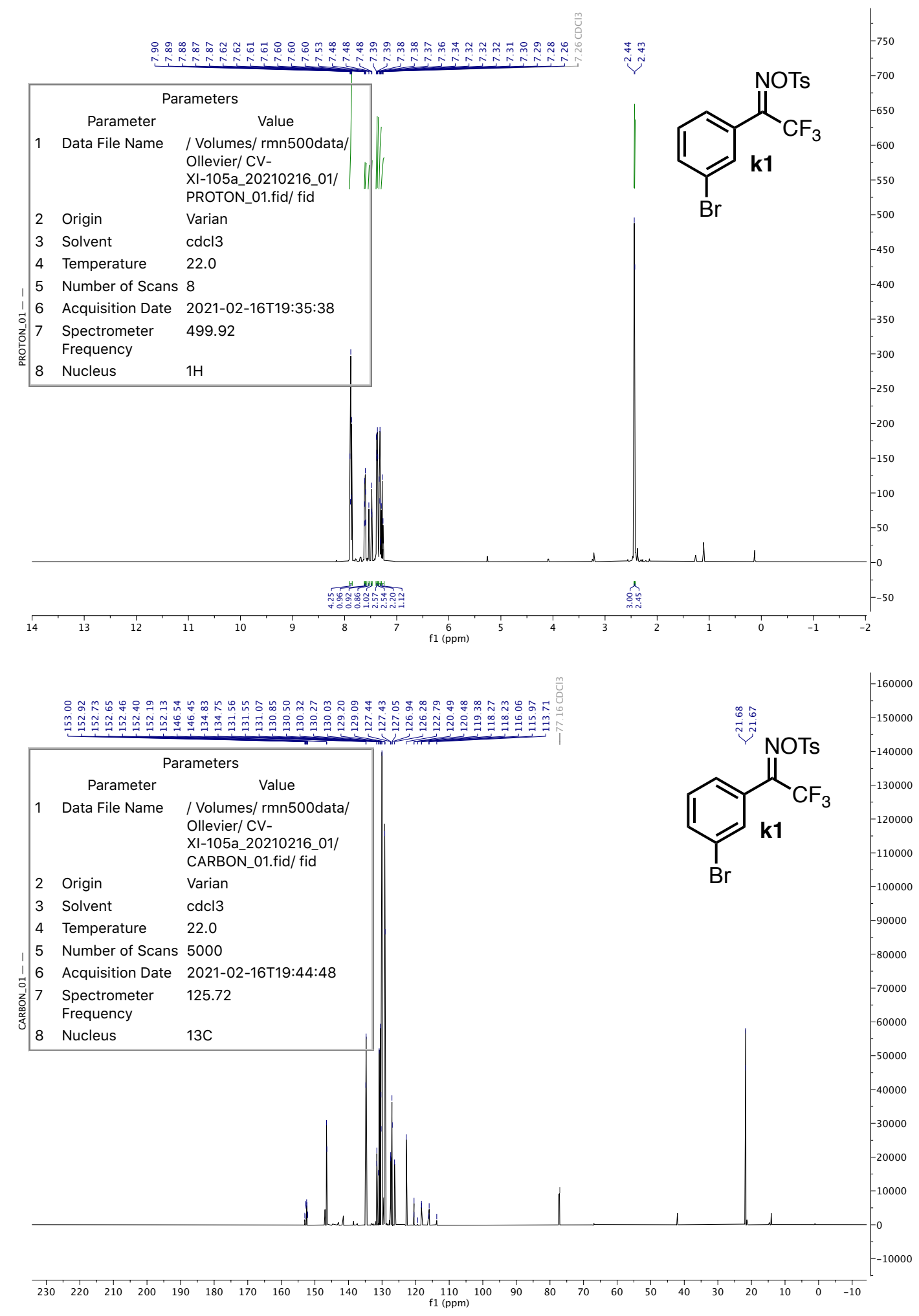


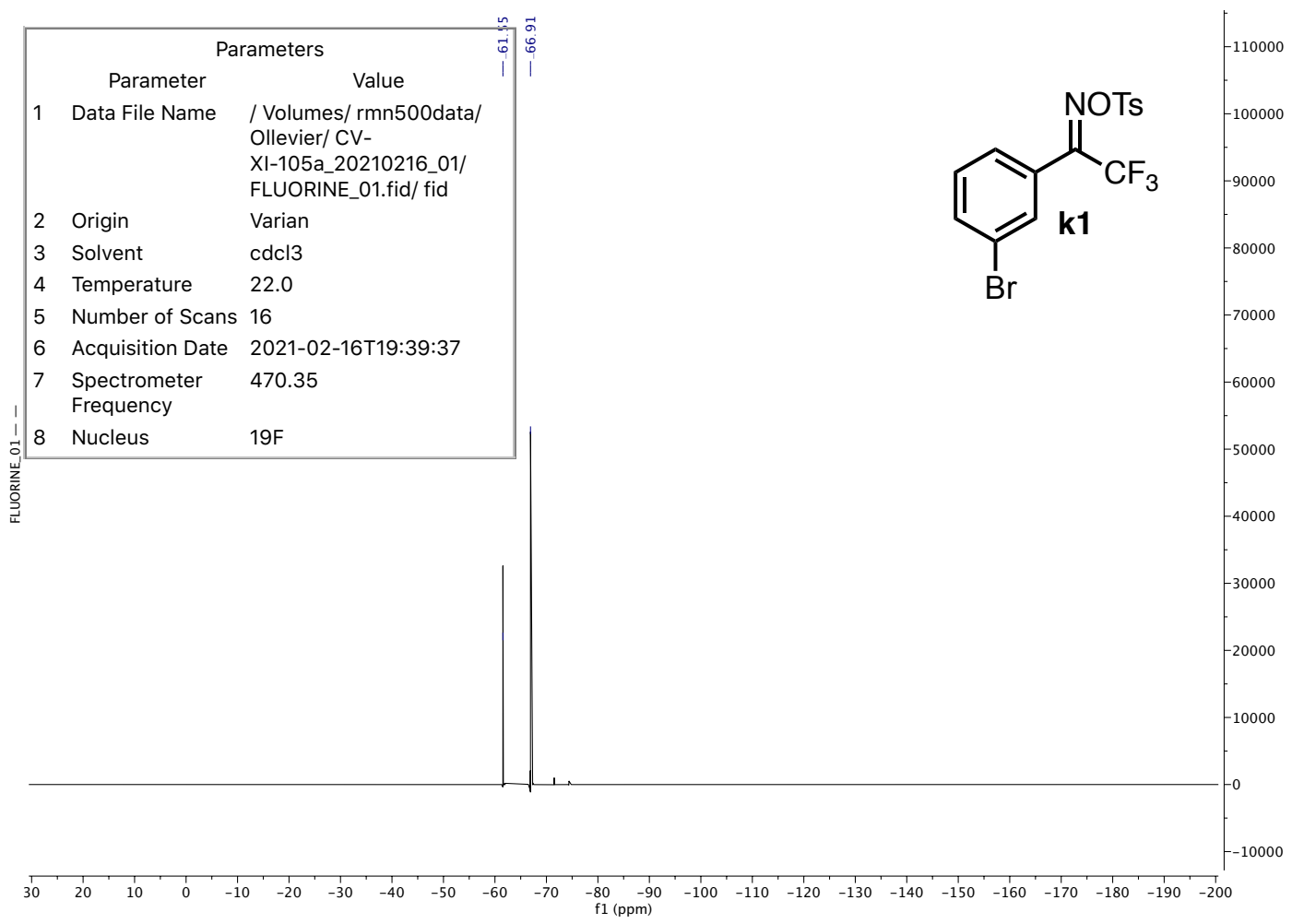

\section{2,2,2-Trifluoro-1-(4-(trifluoromethyl)phenyl)ethan-1-one 0-tosyl oxime I1}

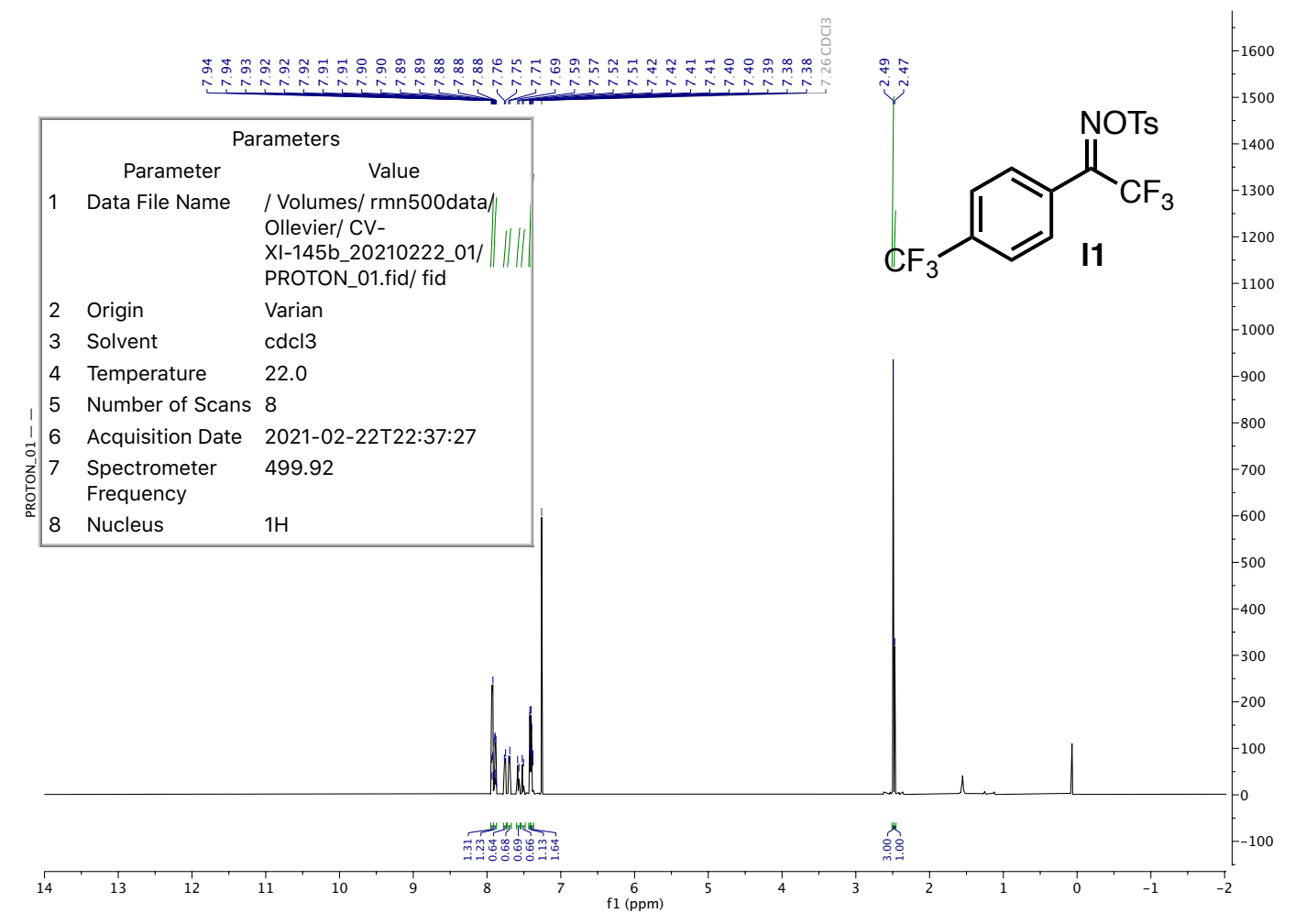




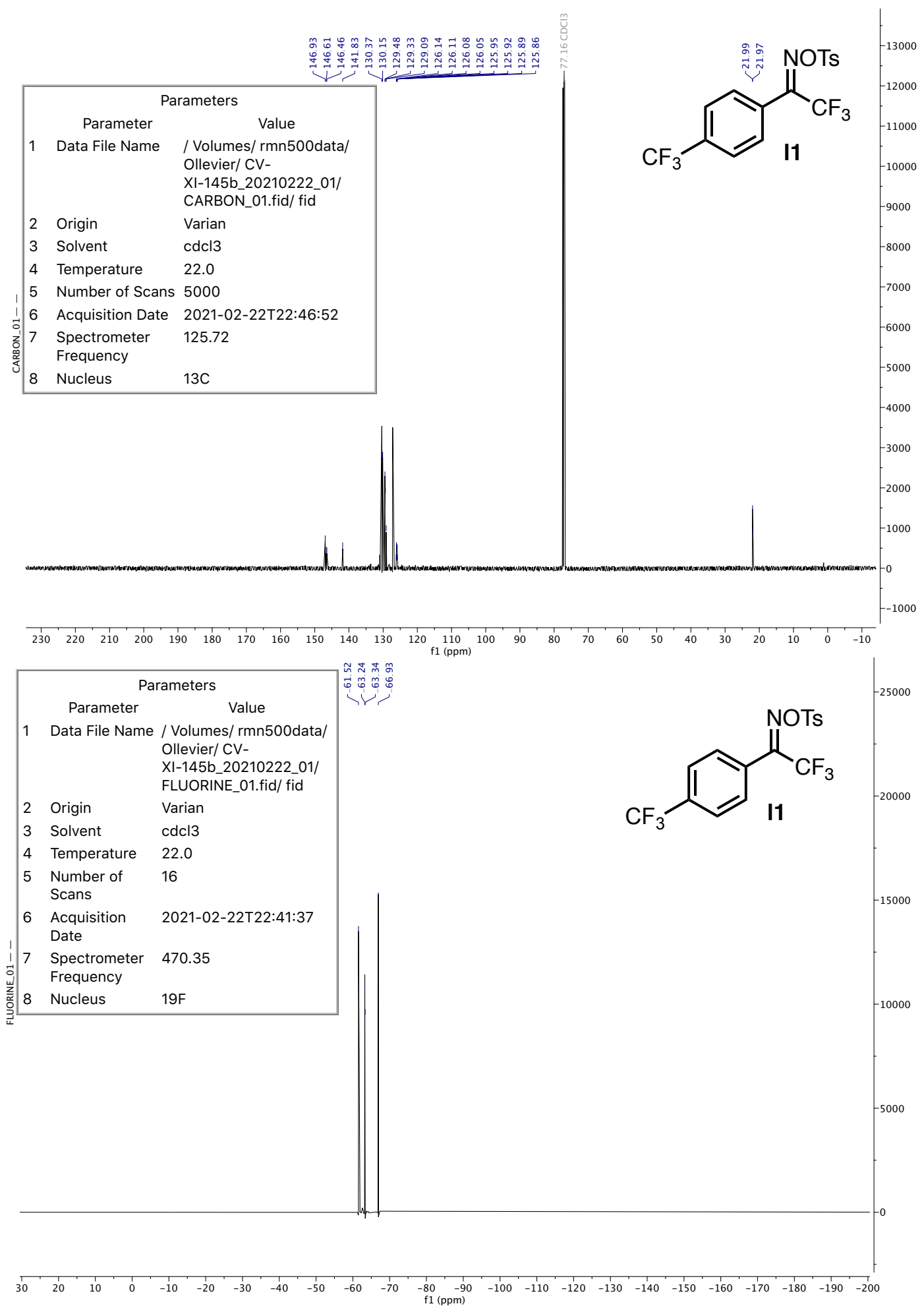




\section{2,2,2-Trifluoro-1-(2-methoxyphenyl)ethan-1-one 0-tosyl oxime m1}

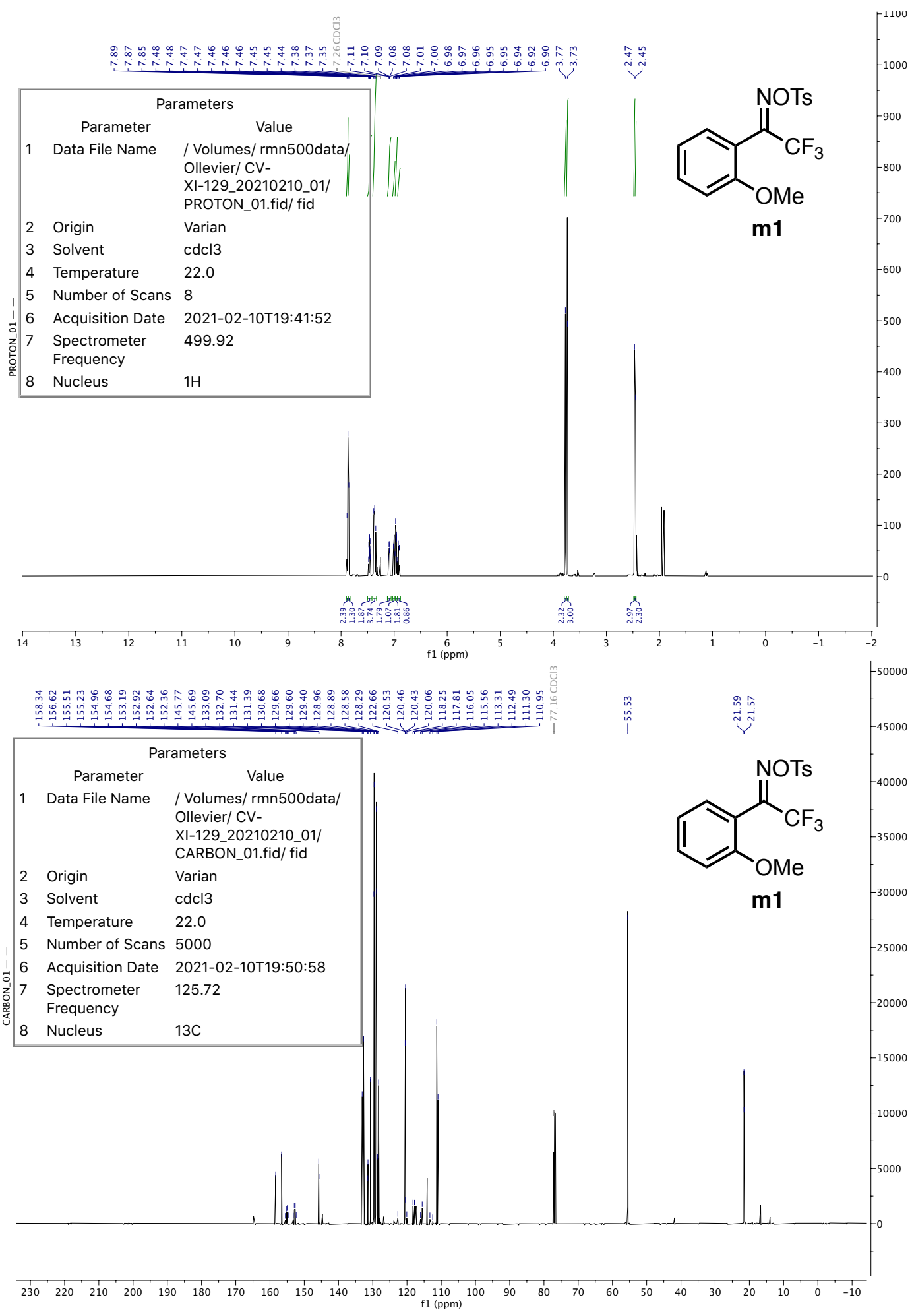




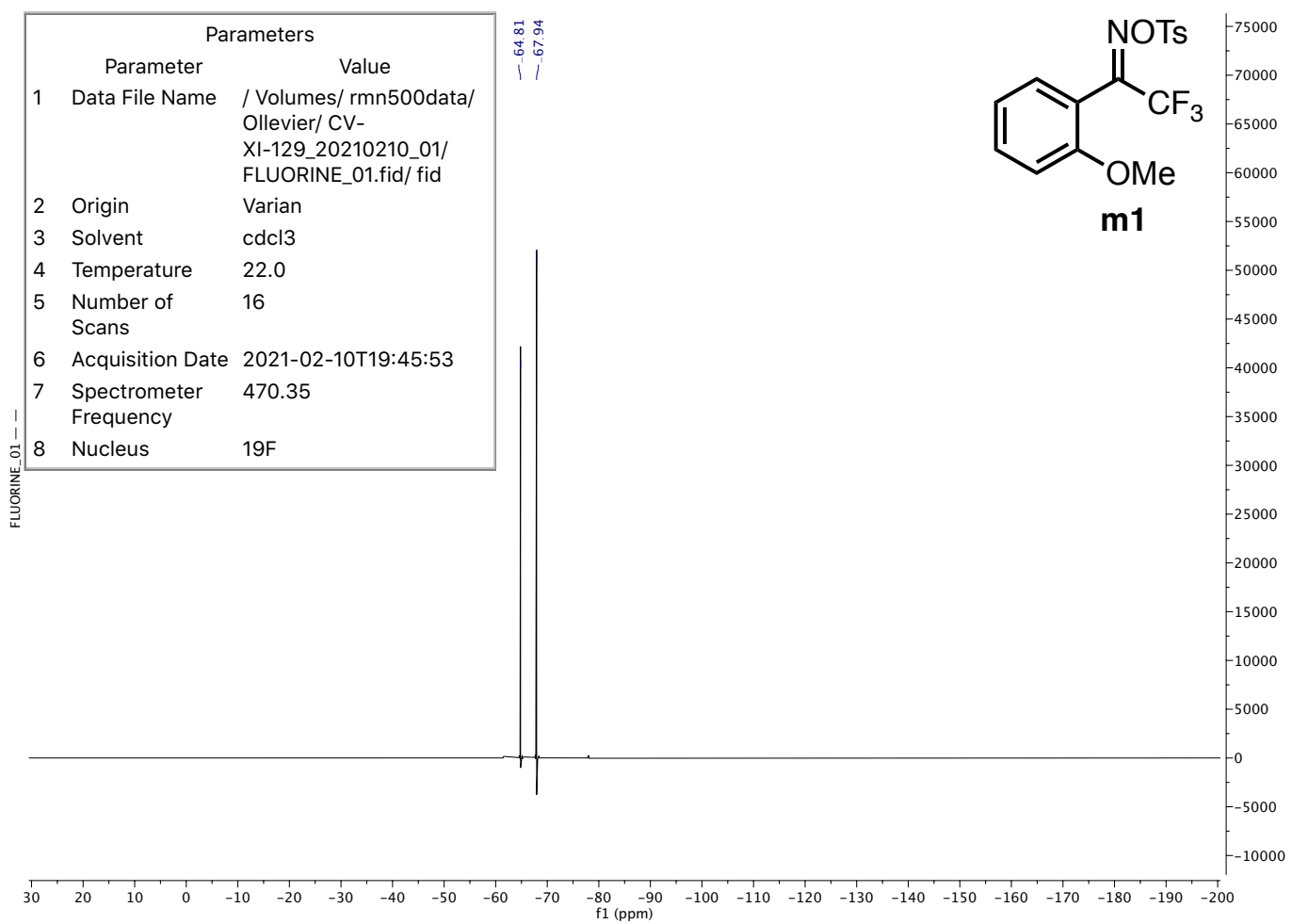

\section{2,2,2-Trifluoro-1-(2-fluorophenyl)ethan-1-one 0-tosyl oxime n1}

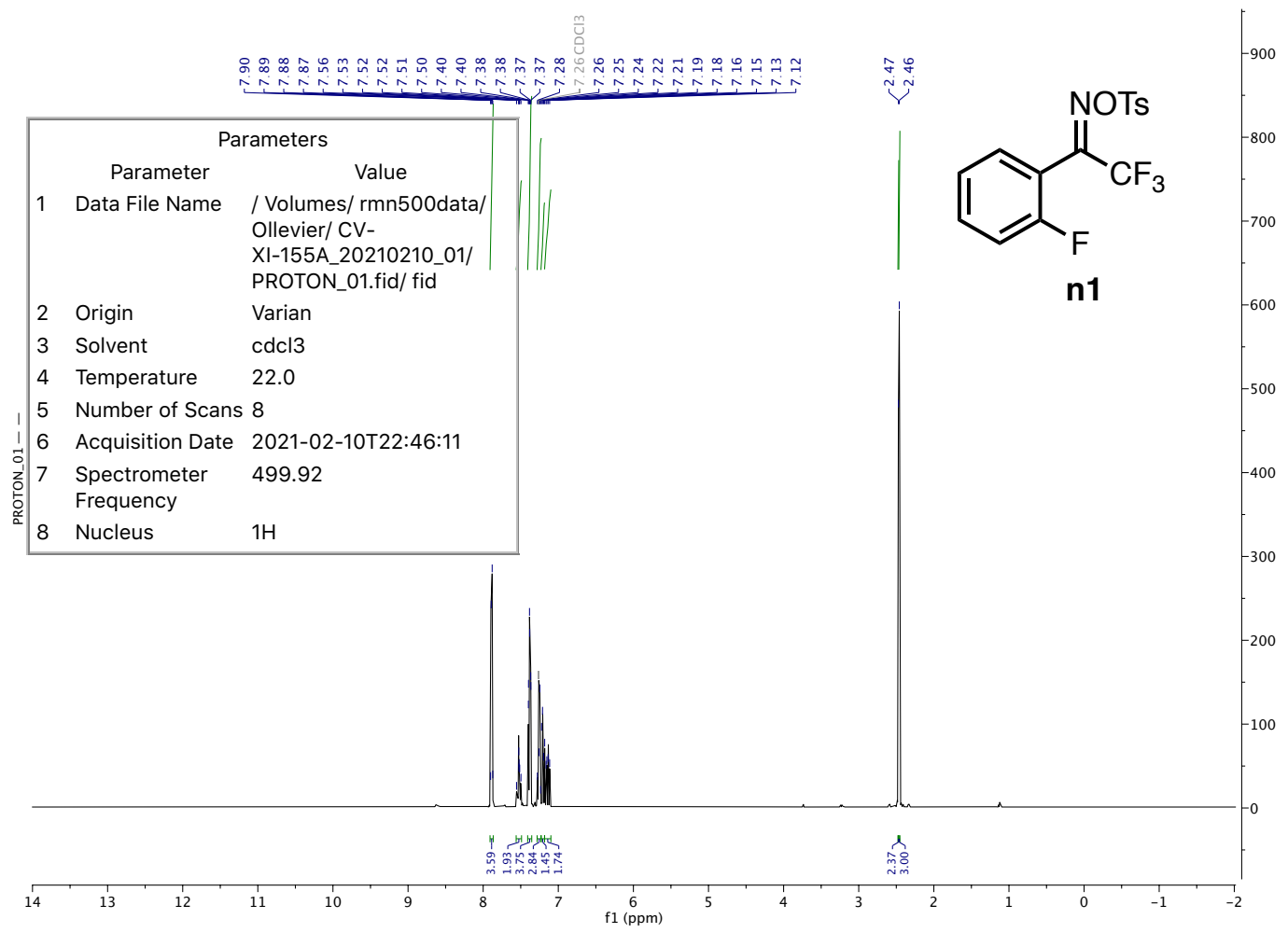




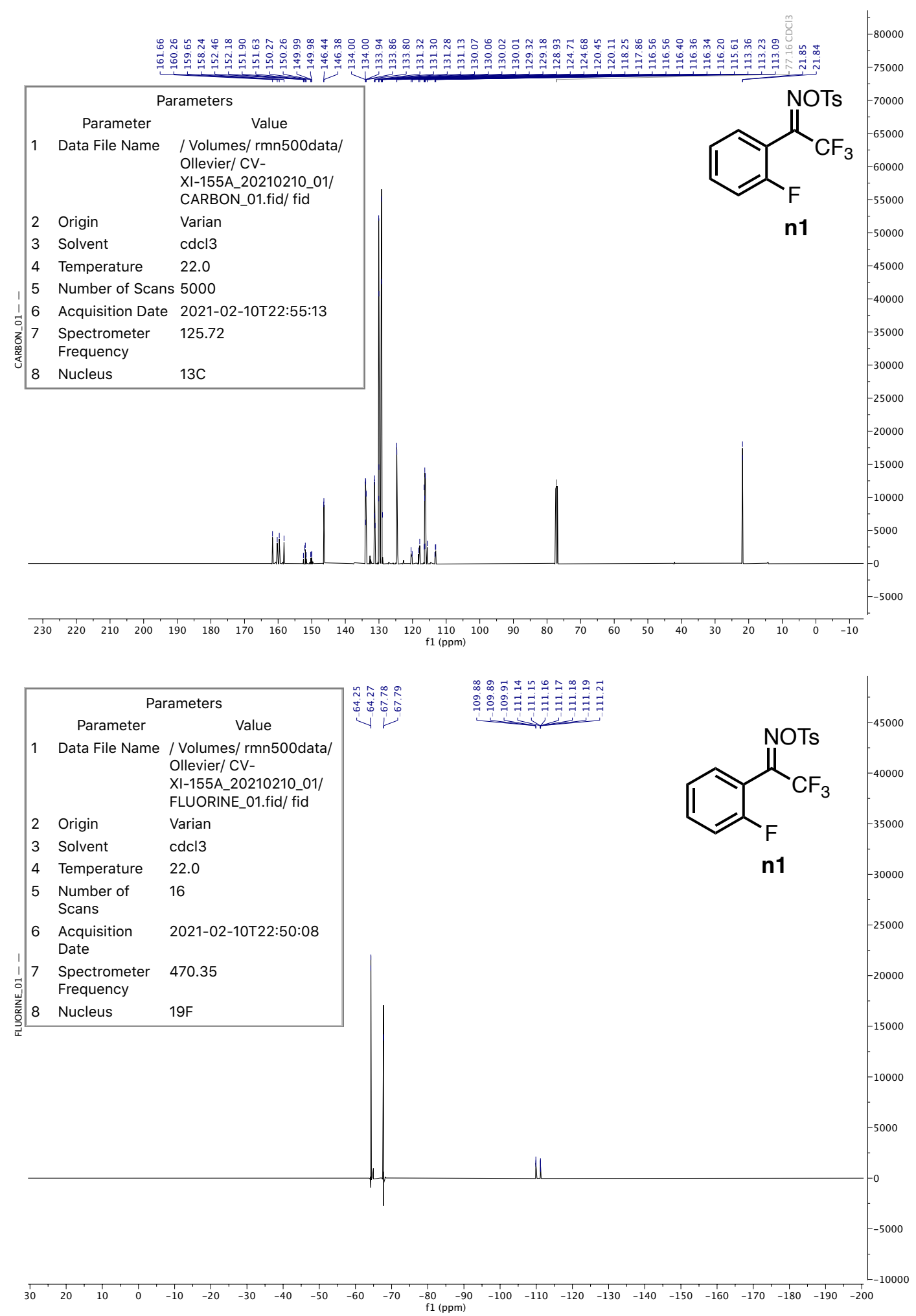




\section{3-(4,4'-Biphenyl)-3-(trifluoromethyl)diaziridine a2}

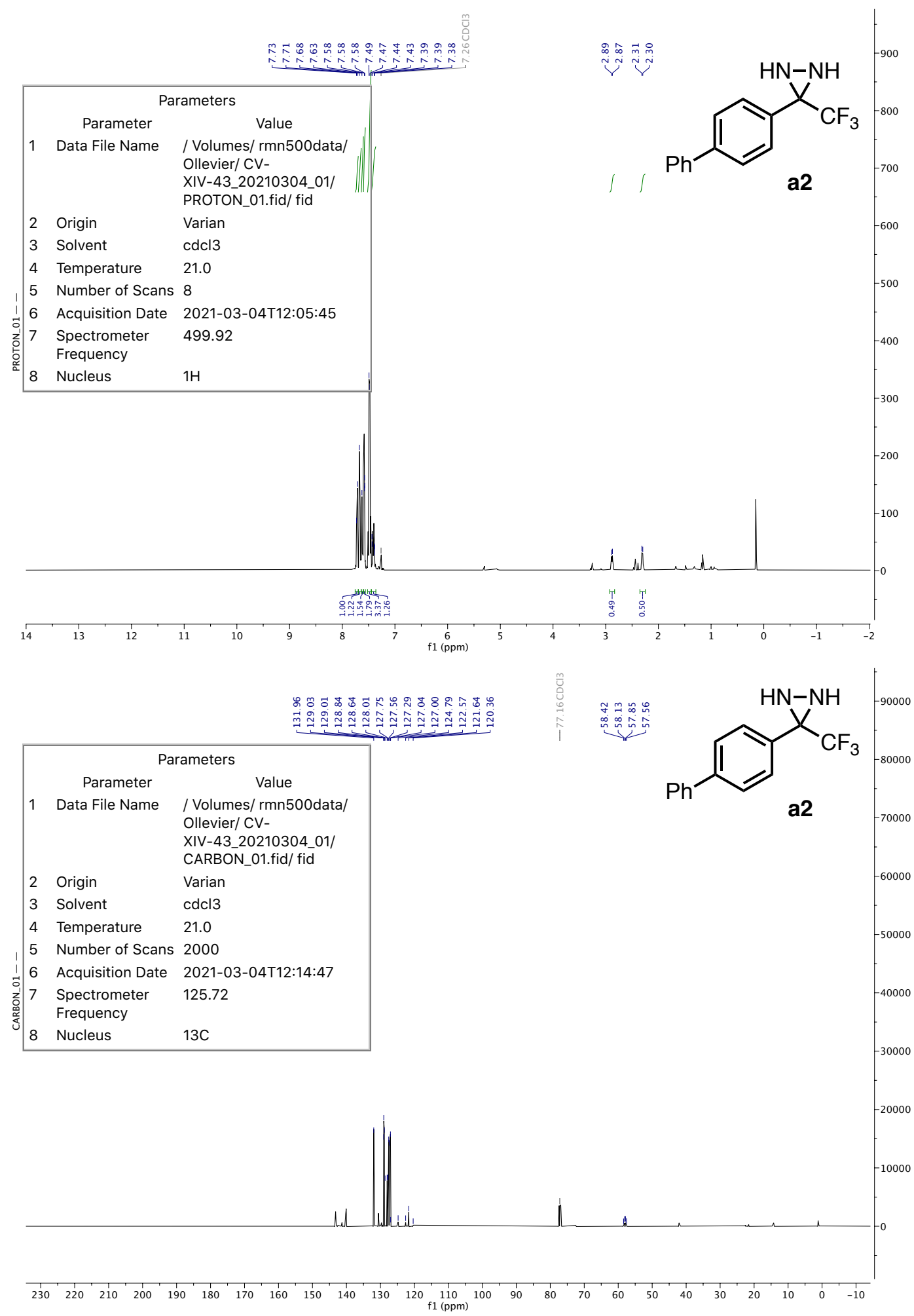




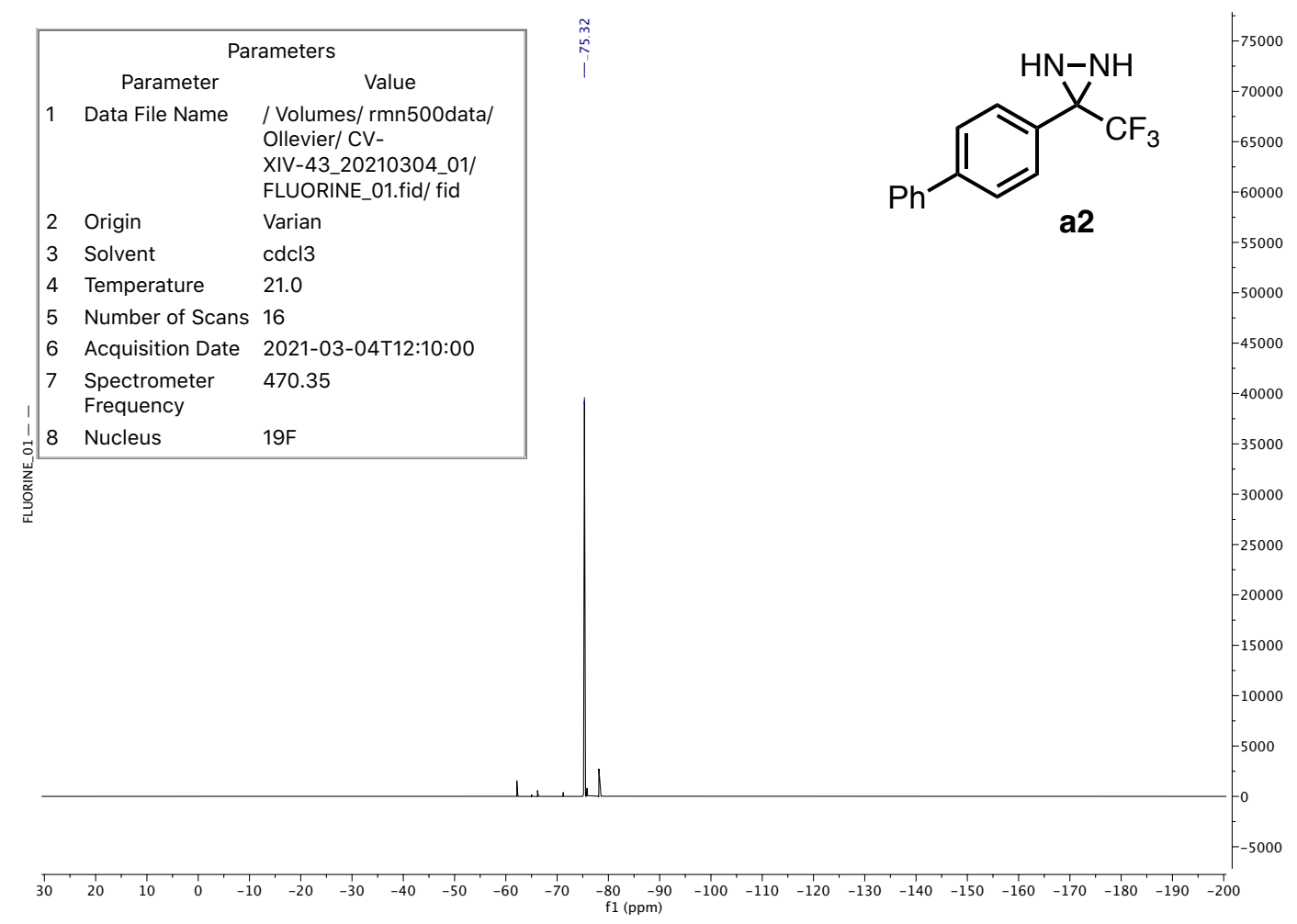

\section{3-(Naphthalin-2-yl)-3-(trifluoromethyl)diaziridine b2}

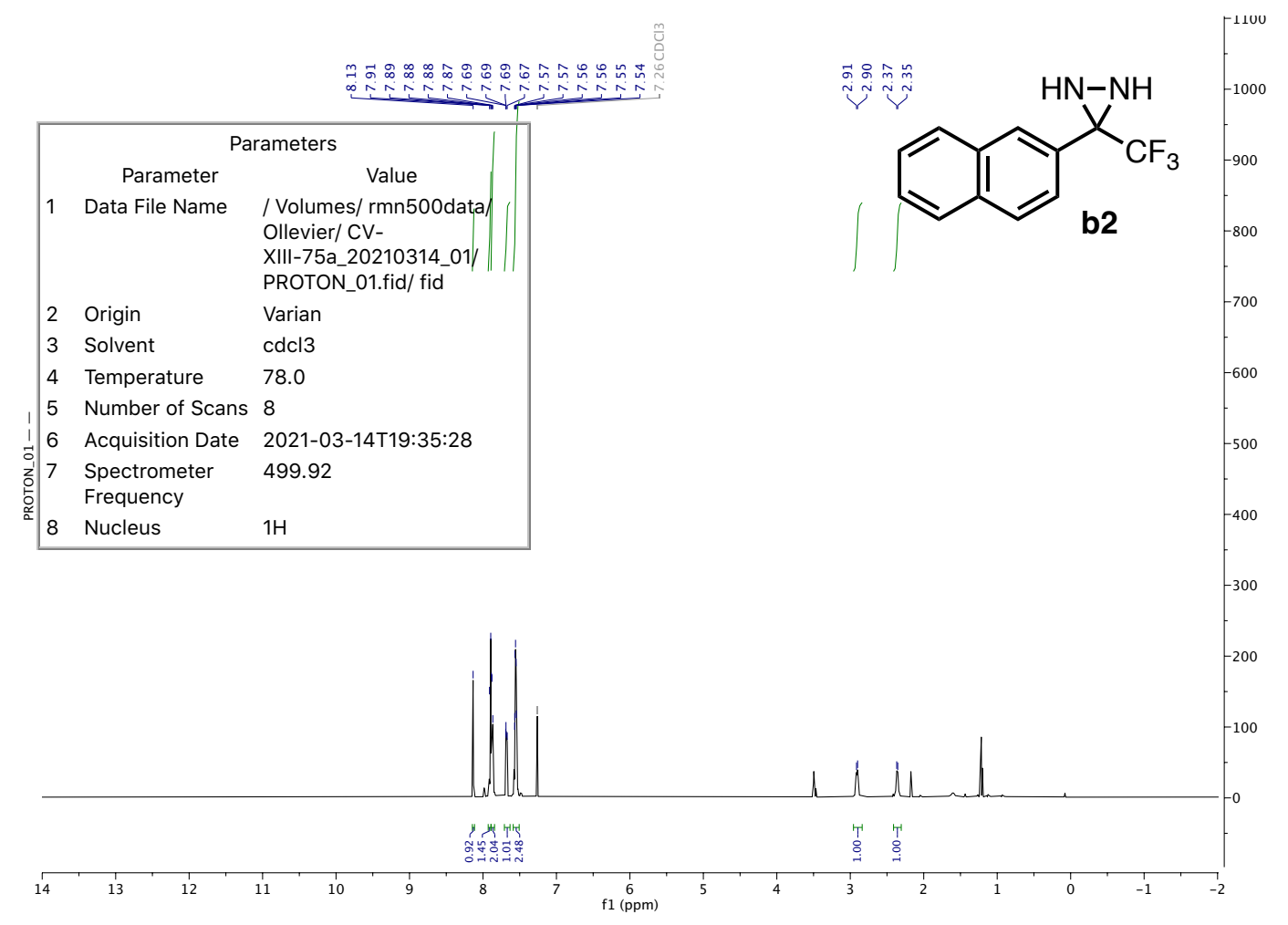




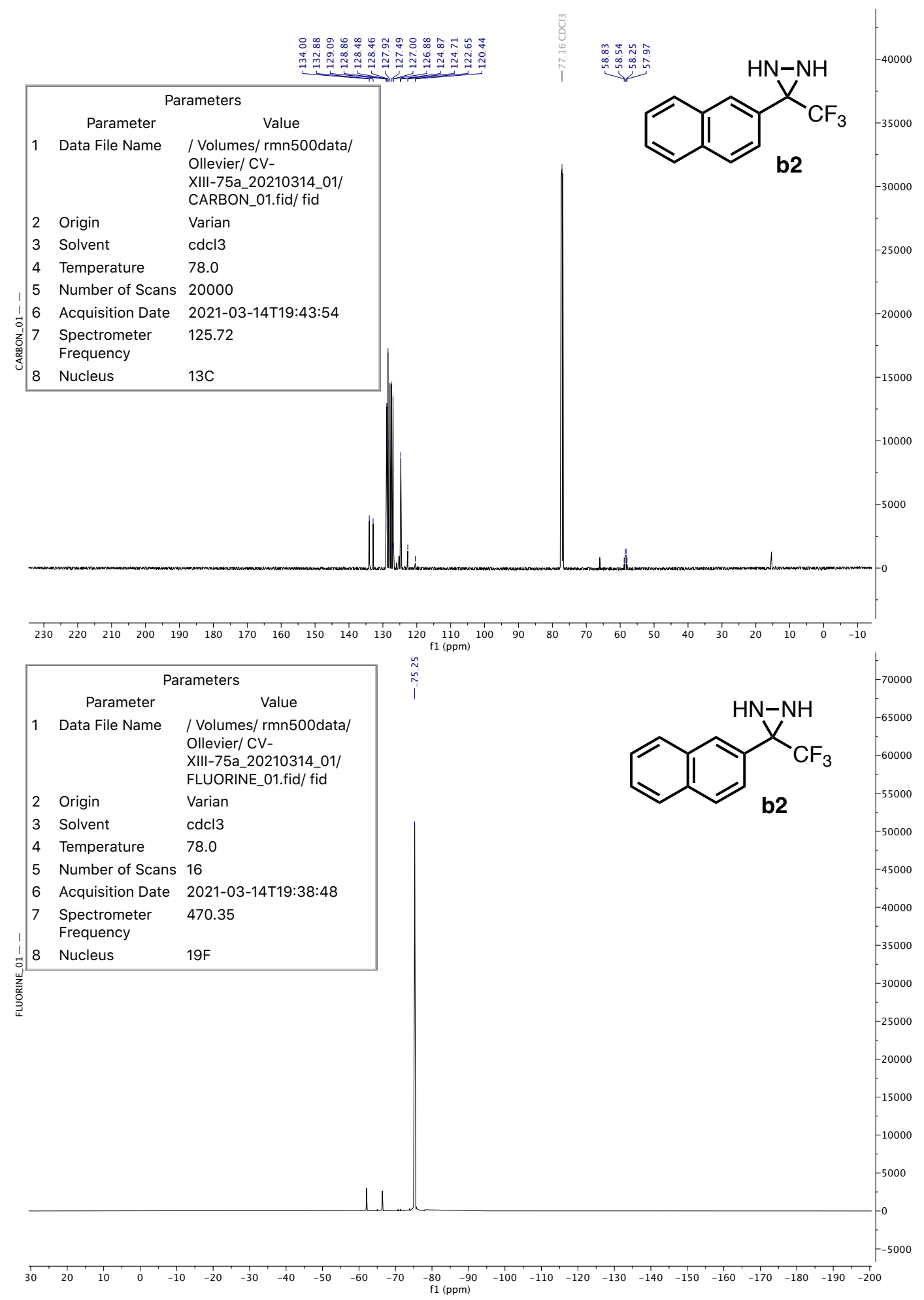


3-(-4-Benzyloxyphenyl)-3-(trifluoromethyl)diaziridine c2

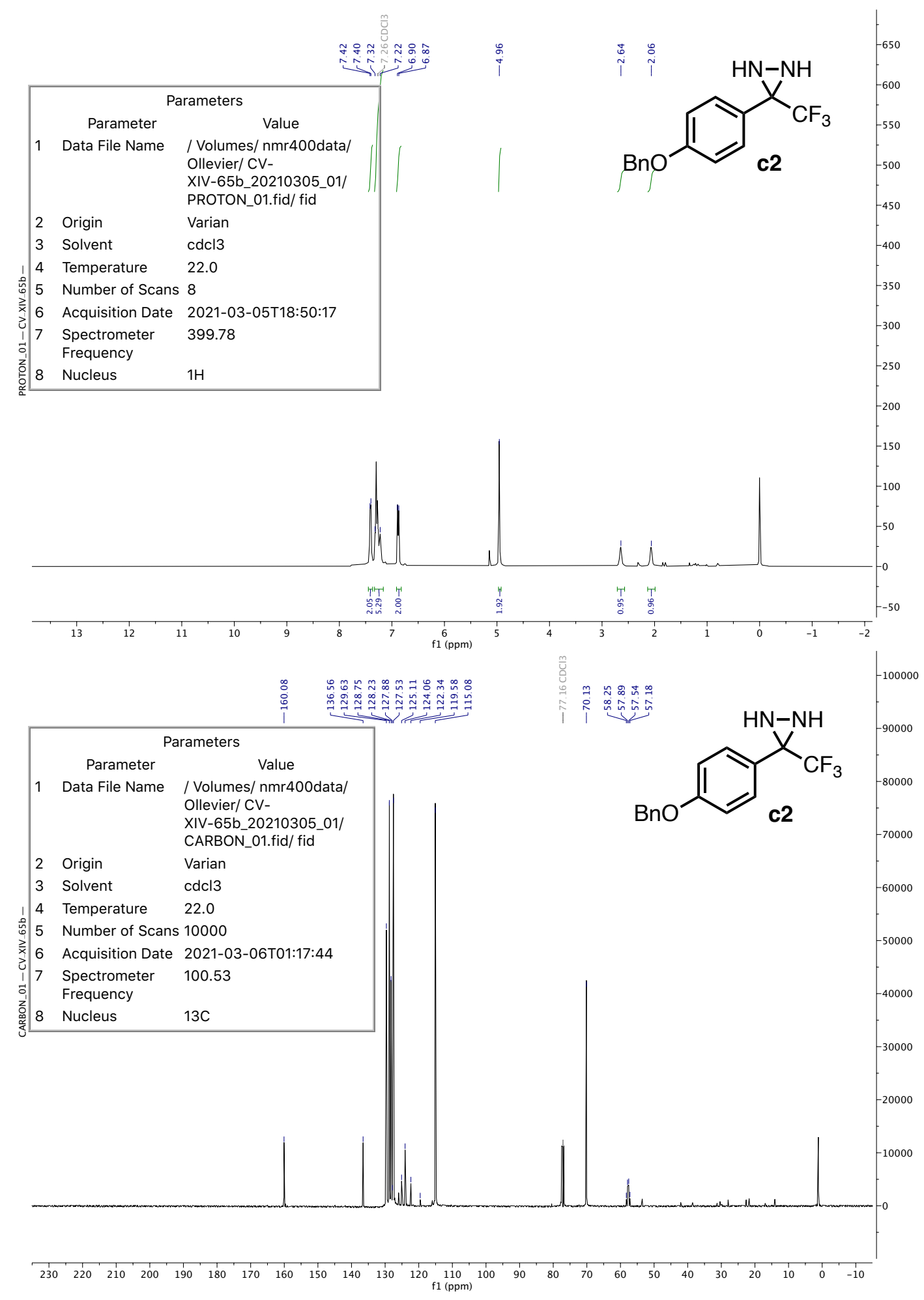




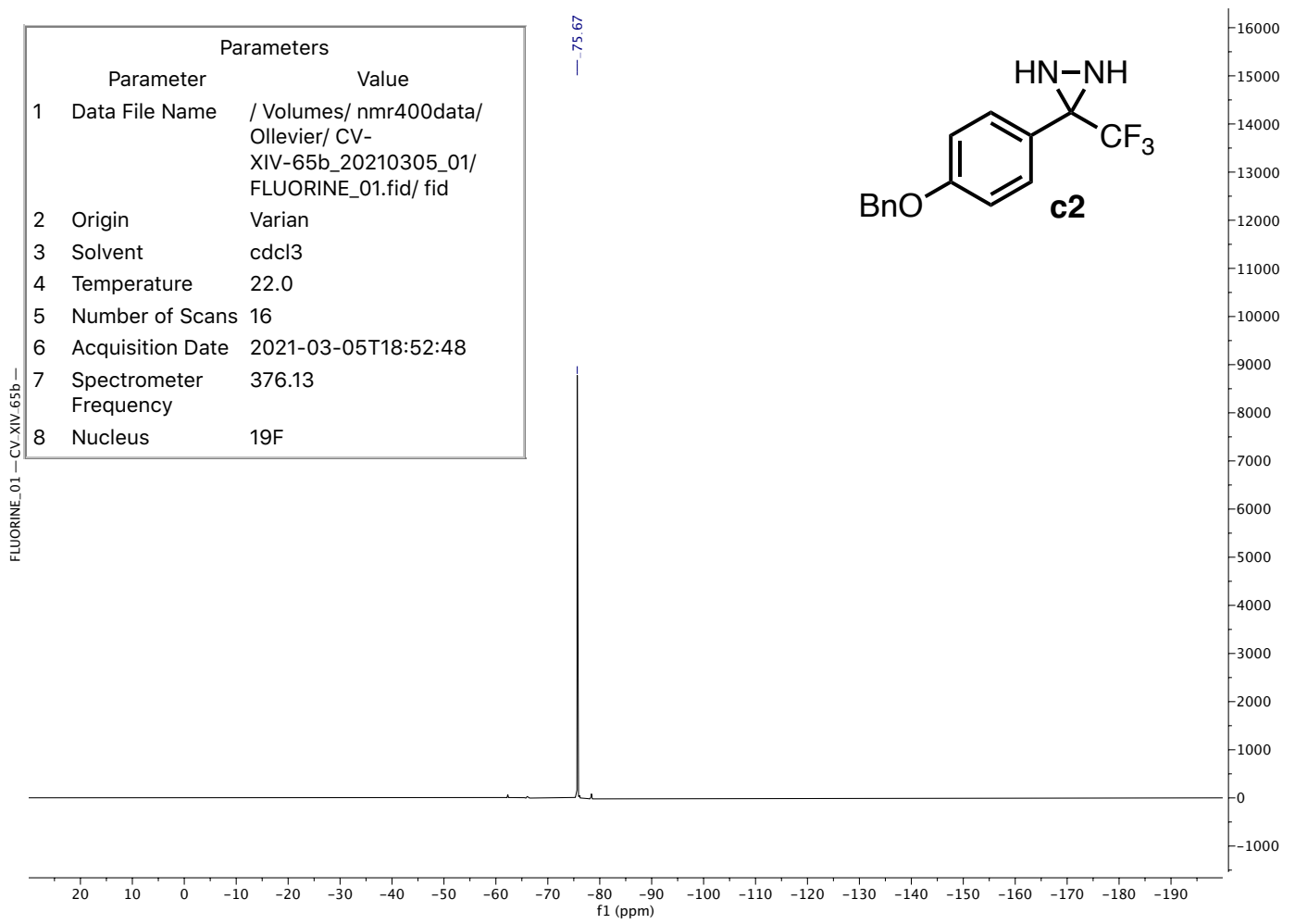

\section{Bis-(4,4'-biphenyl)-3-(trifluoromethyl)diaziridine d2}

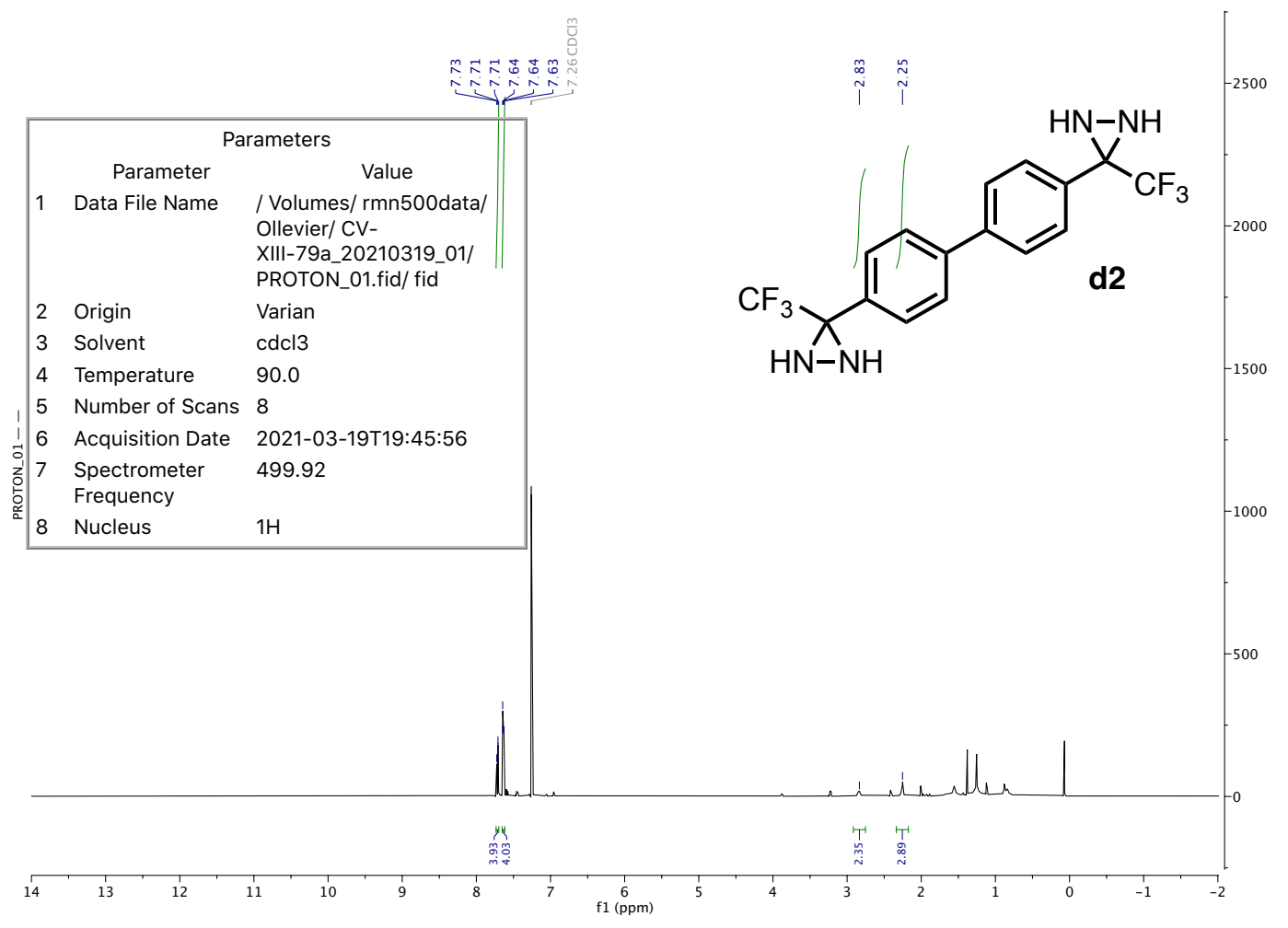




\begin{tabular}{|c|c|c|}
\hline \multicolumn{3}{|c|}{ Parameters } \\
\hline & Parameter & Value \\
\hline 1 & Data File Name & $\begin{array}{l}\text { / Volumes/ rmn500data/ } \\
\text { Ollevier/ CV- } \\
\text { XIII-79a_20210319_01/ } \\
\text { CARBON_01.fid/ fid }\end{array}$ \\
\hline 2 & Origin & Varian \\
\hline 3 & Solvent & cdcl3 \\
\hline 4 & Temperature & 90.0 \\
\hline 5 & Number of Scans & 20000 \\
\hline 6 & Acquisition Date & 2021-03-19T19:55:01 \\
\hline 7 & $\begin{array}{l}\text { Spectrometer } \\
\text { Frequency }\end{array}$ & 125.72 \\
\hline 8 & Nucleus & $13 \mathrm{C}$ \\
\hline
\end{tabular}
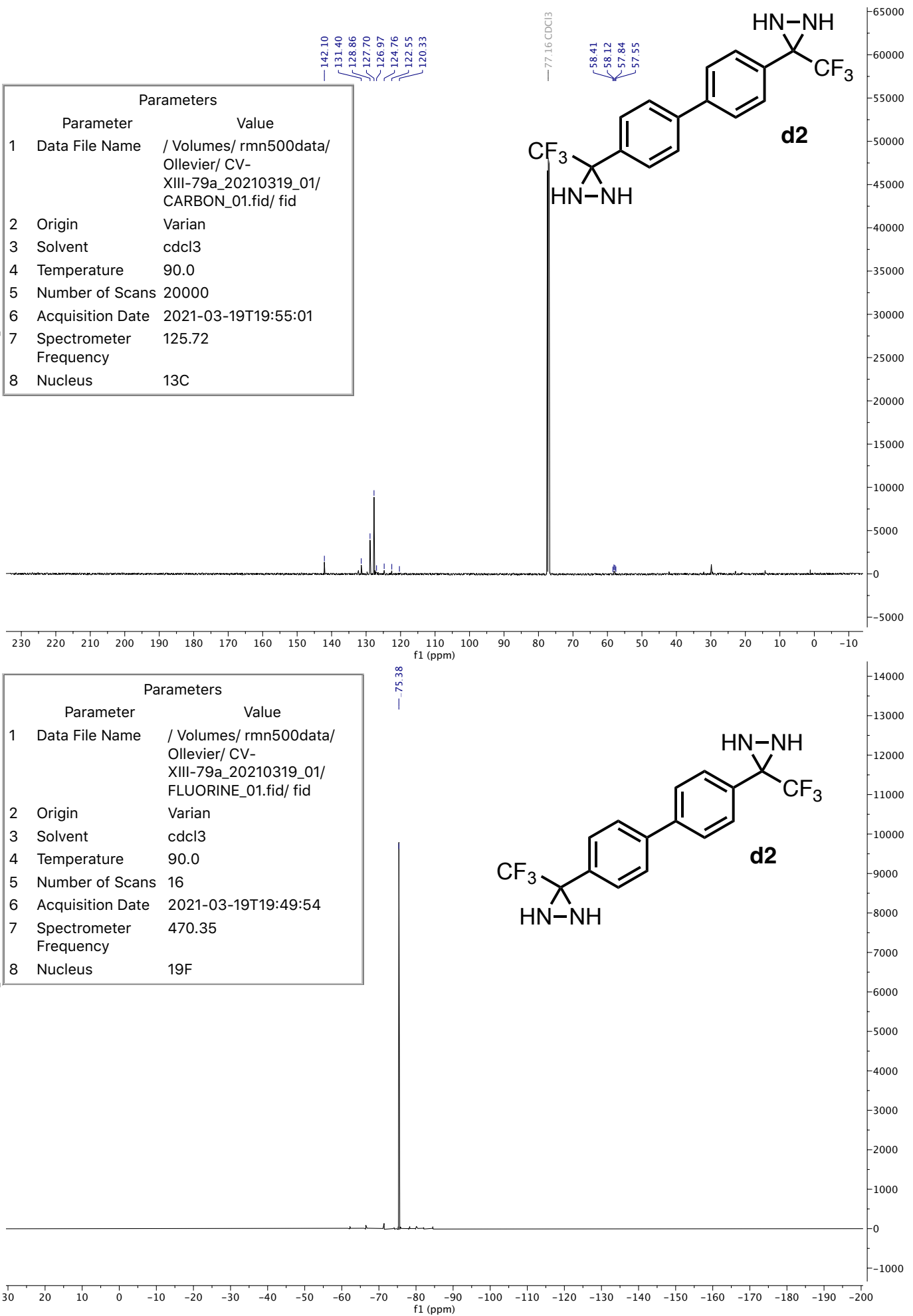
3-(p-Tolyl)-3-(trifluoromethyl)diaziridine e2

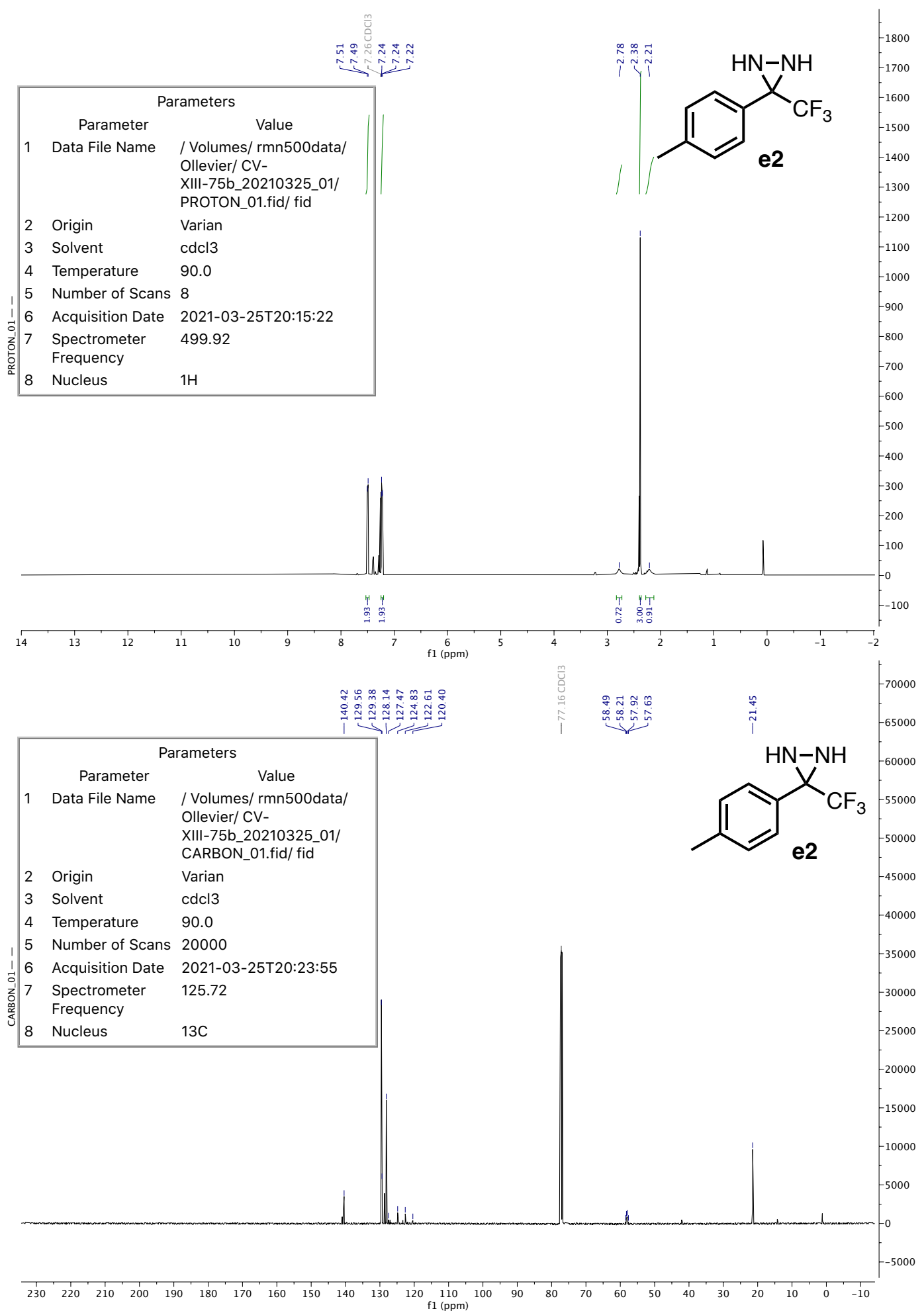




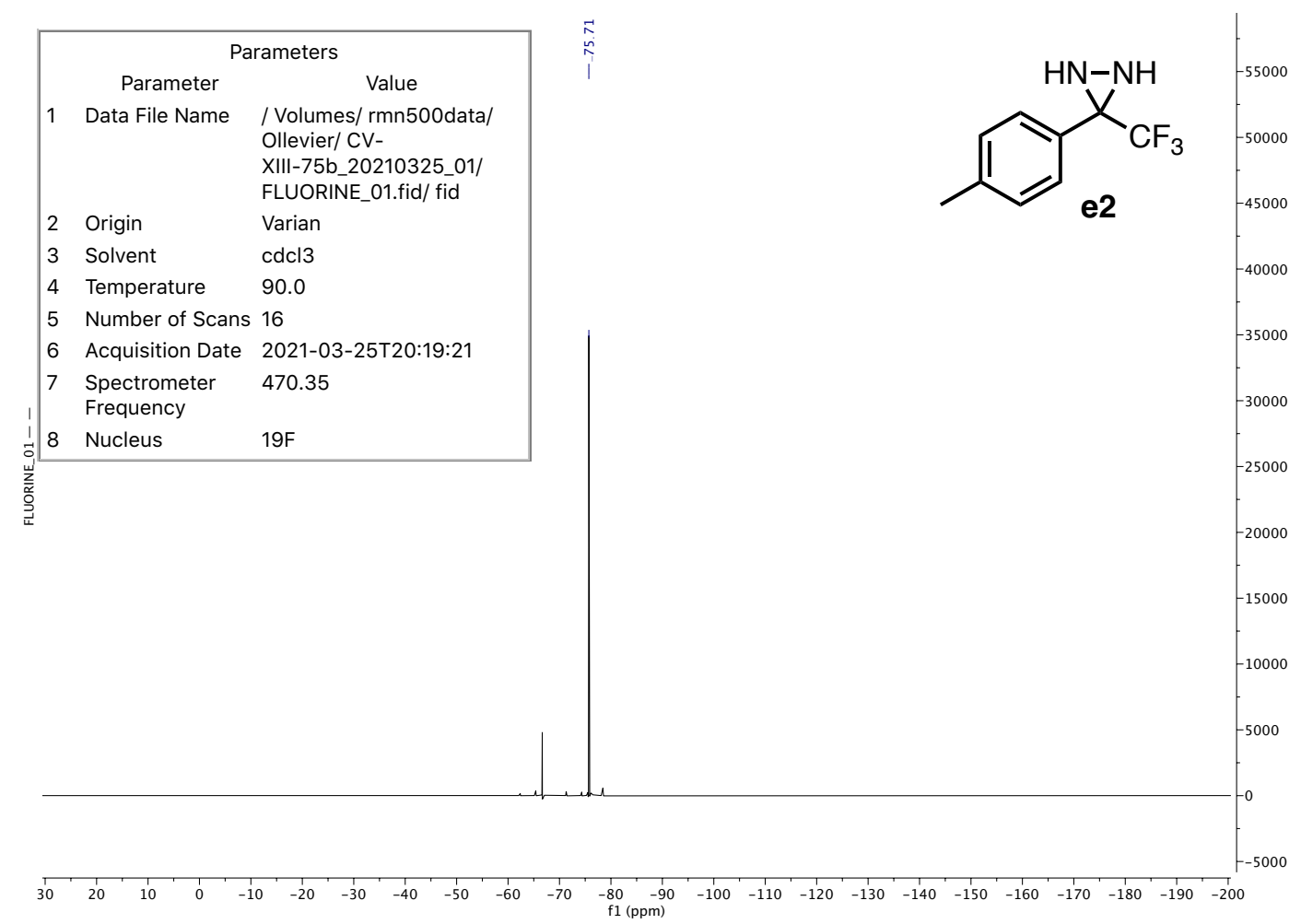

\section{3-(4-Tert-butylphenyl)-3-(trifluoromethyl)diaziridine f2}

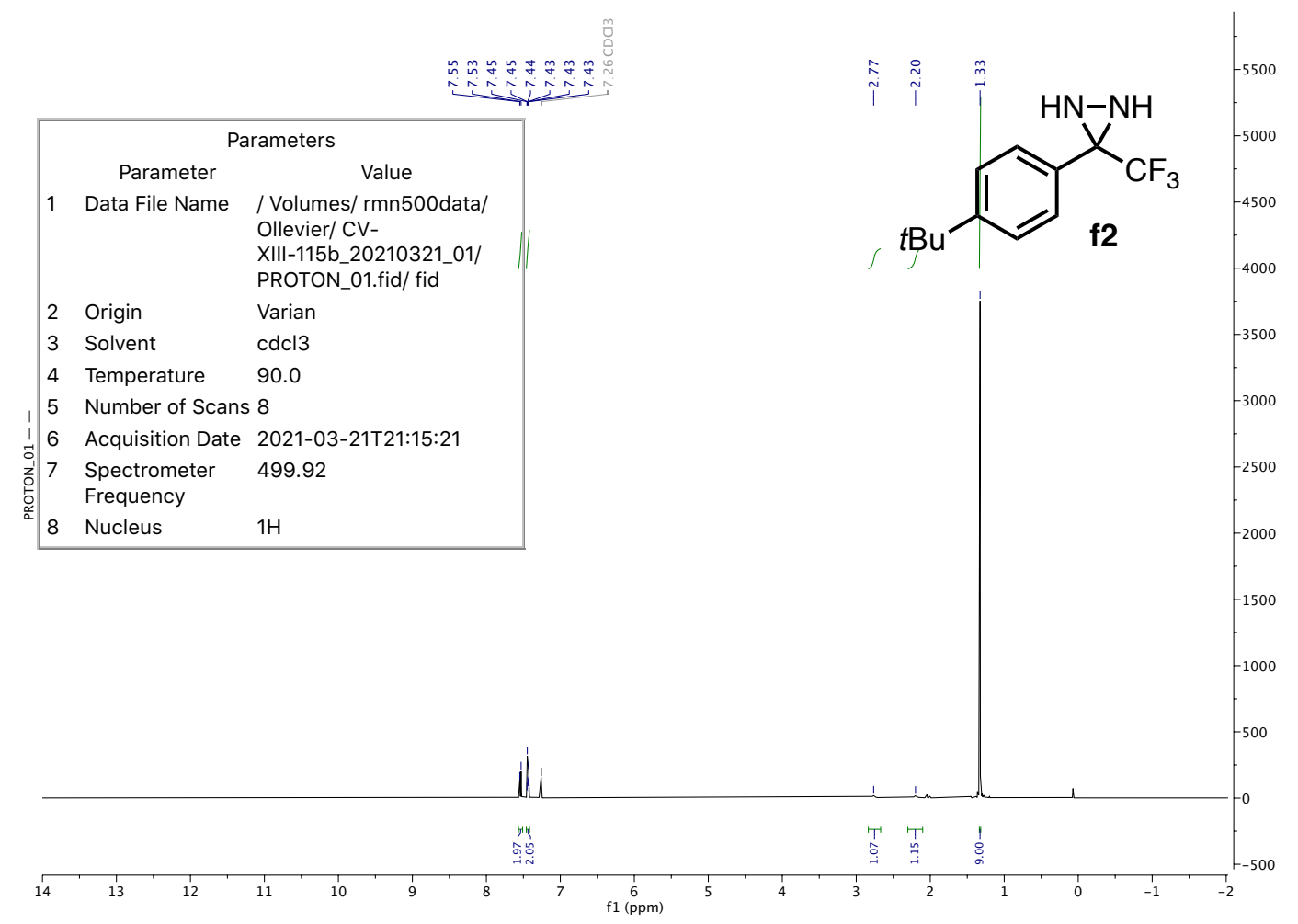




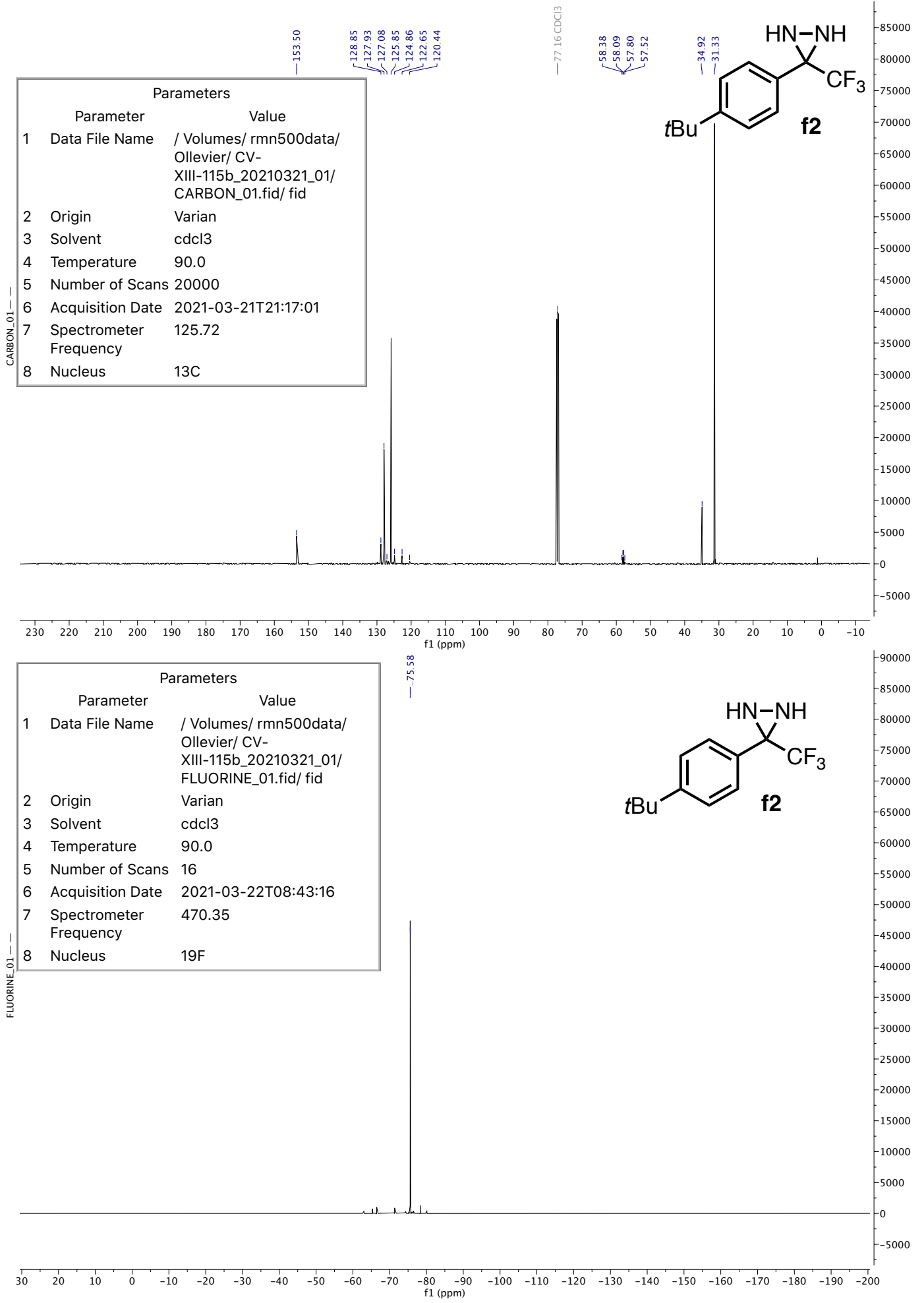




\section{3-(4-Methoxyphenyl)-3-(trifluoromethyl)diaziridine g2}

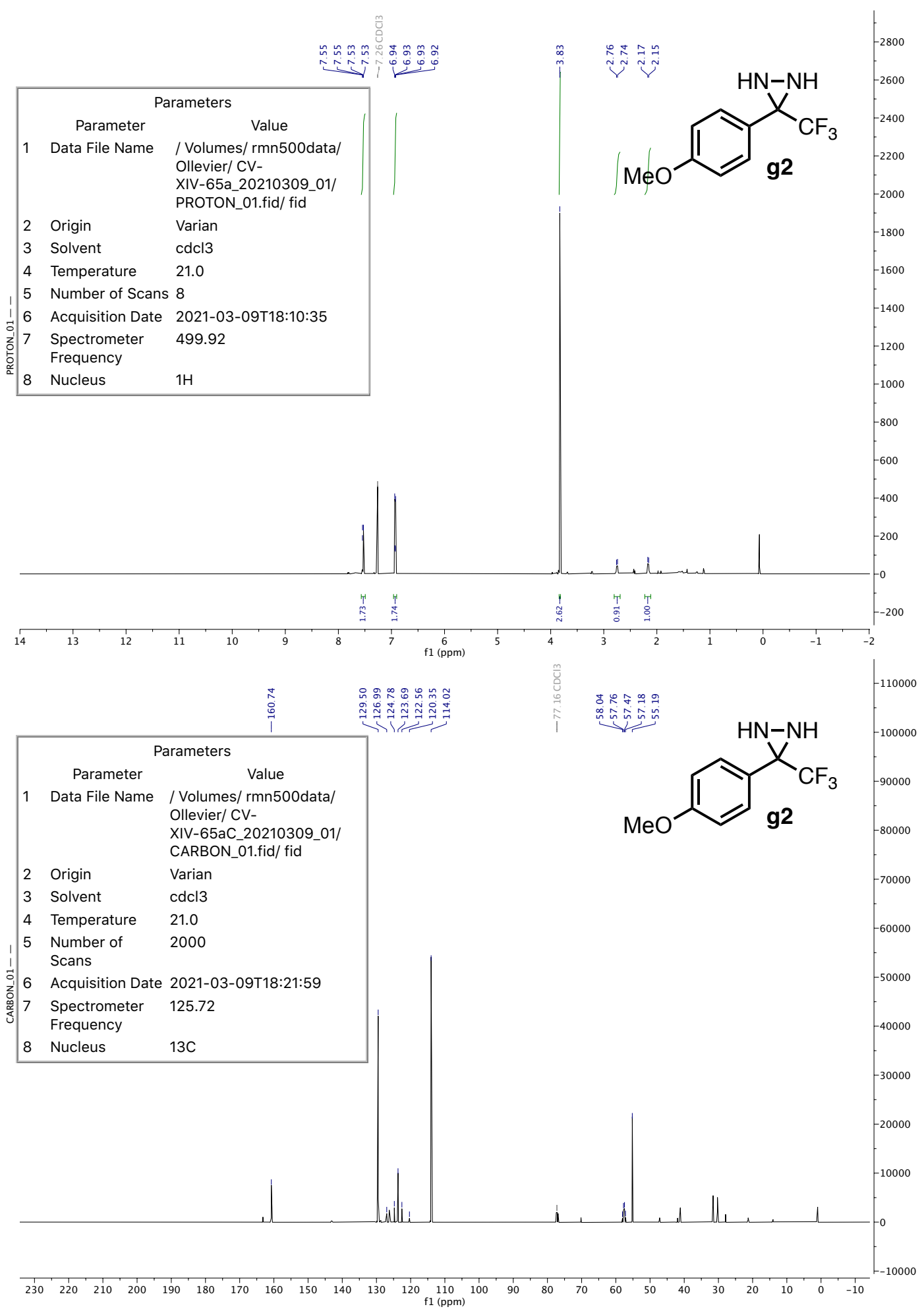




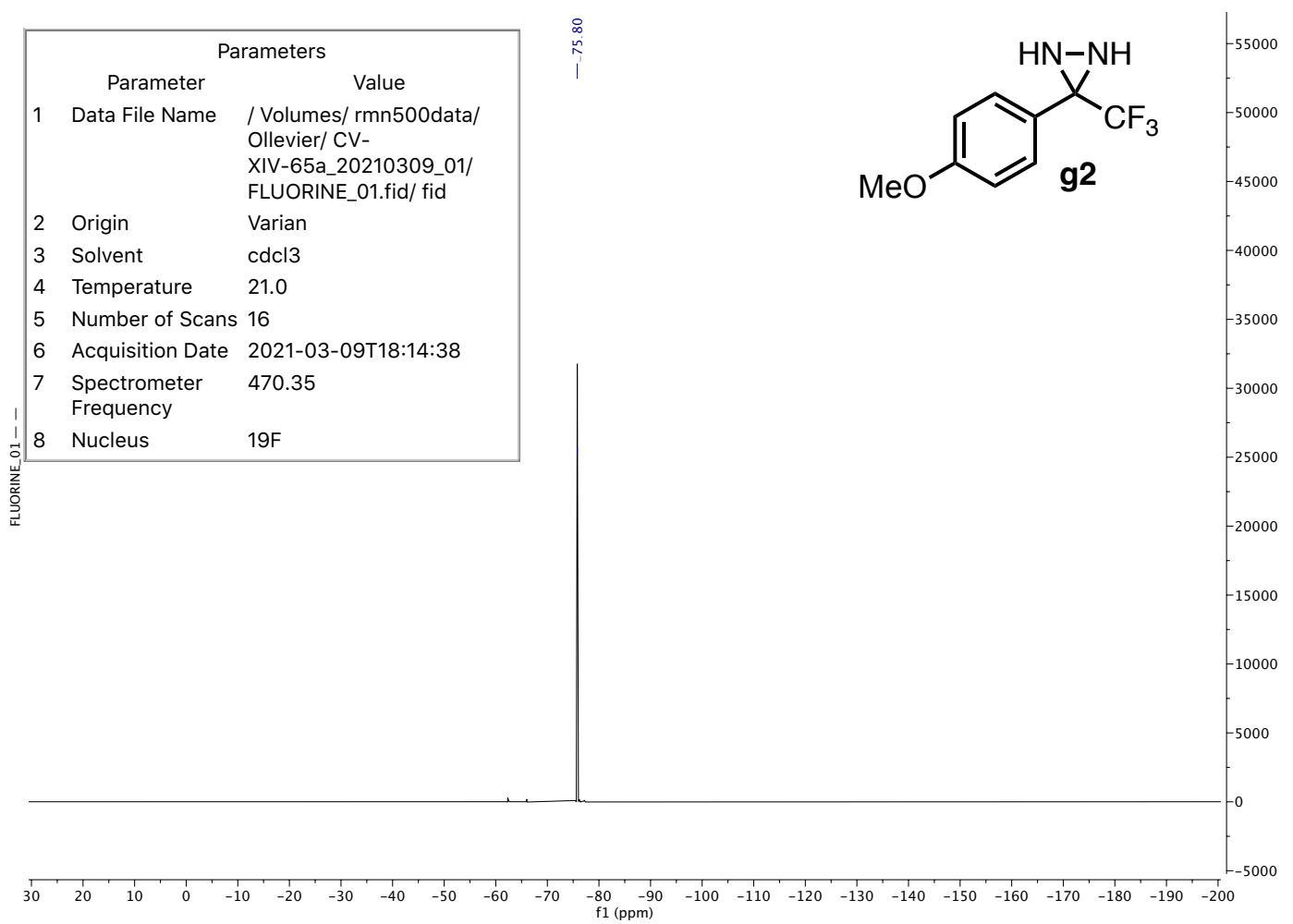

3-Phenyl-3-(trifluoromethyl)diaziridine h2

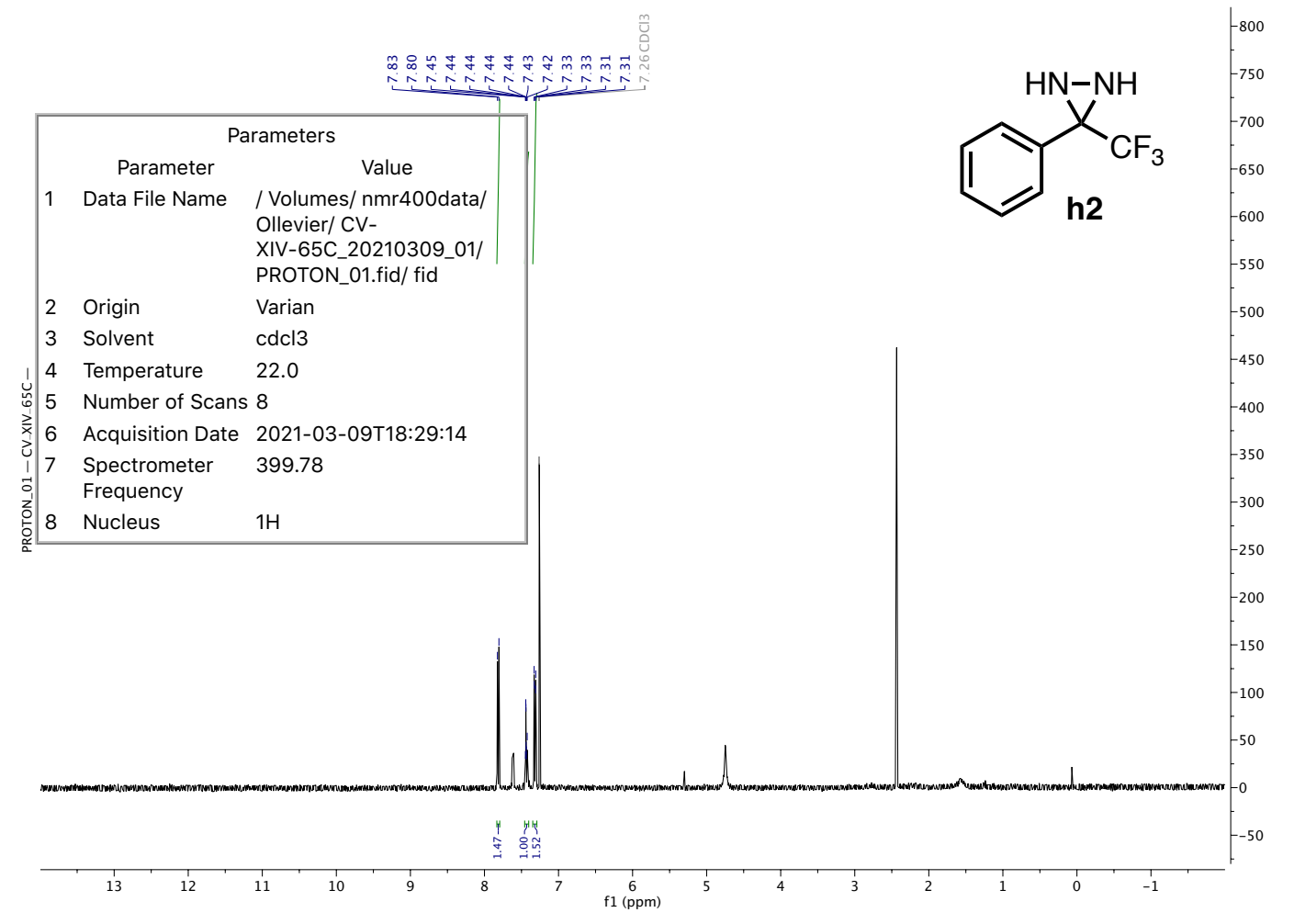




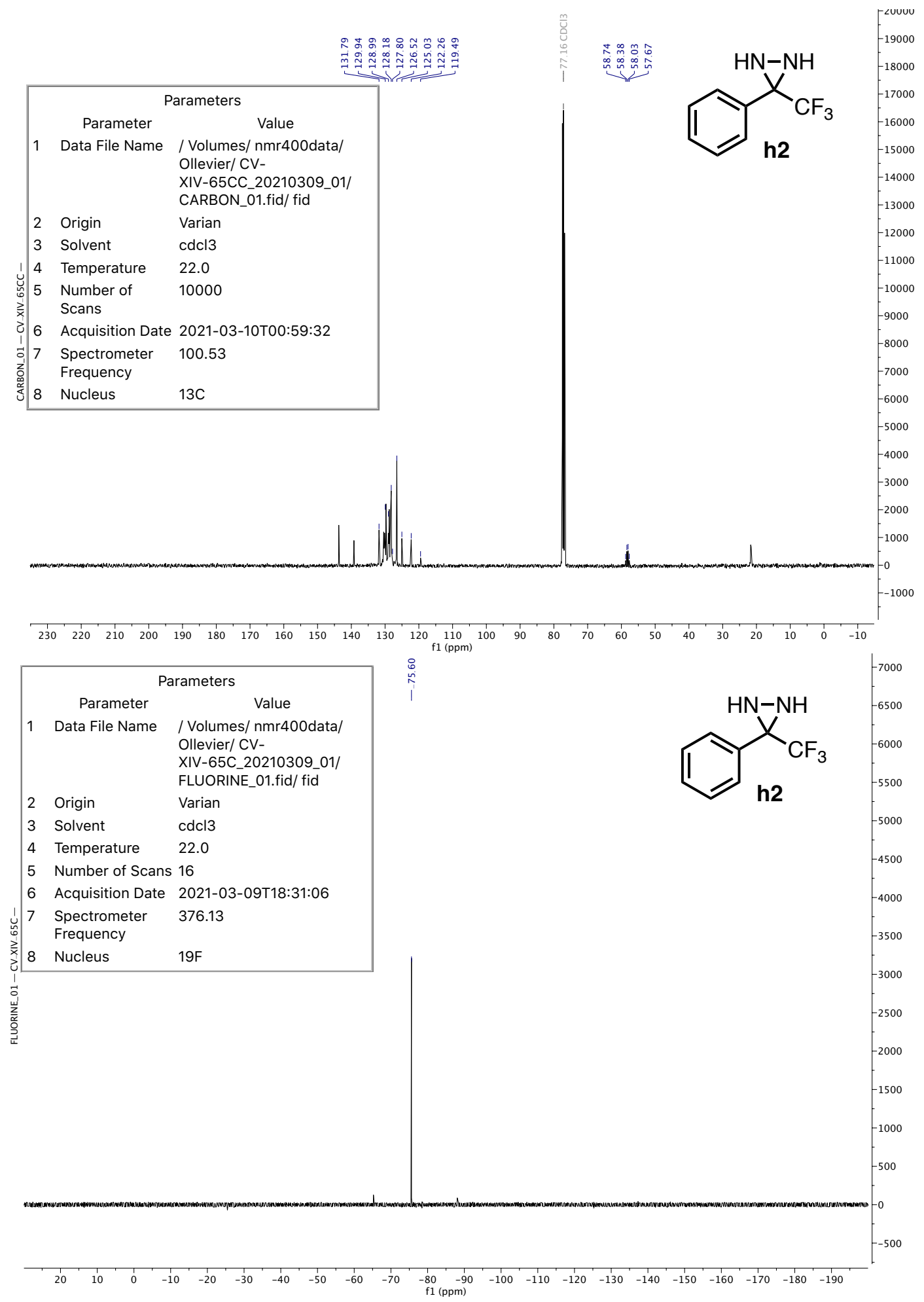




\section{3-(4-Fluorophenyl)-3-(trifluoromethyl)diaziridine i2}

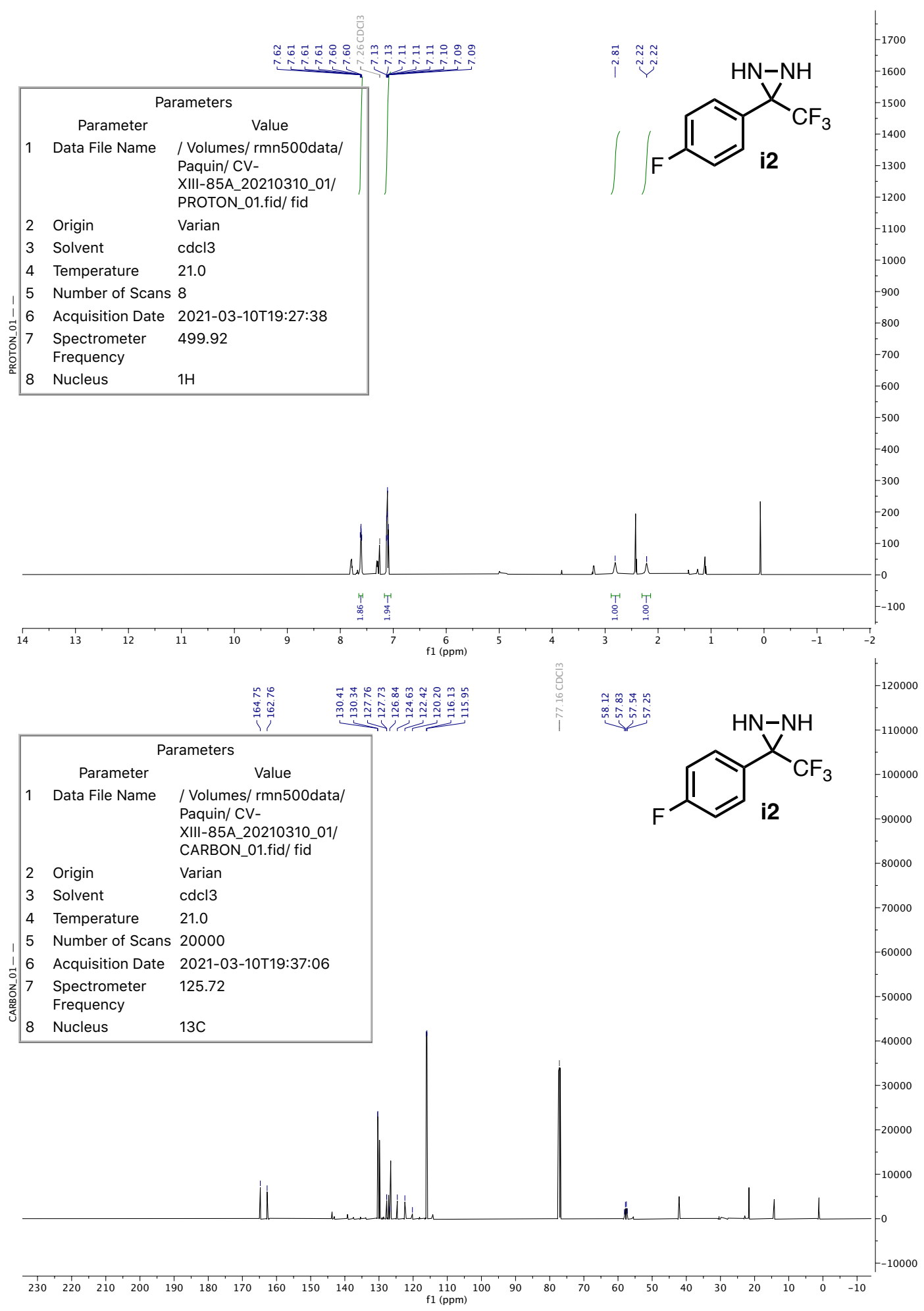




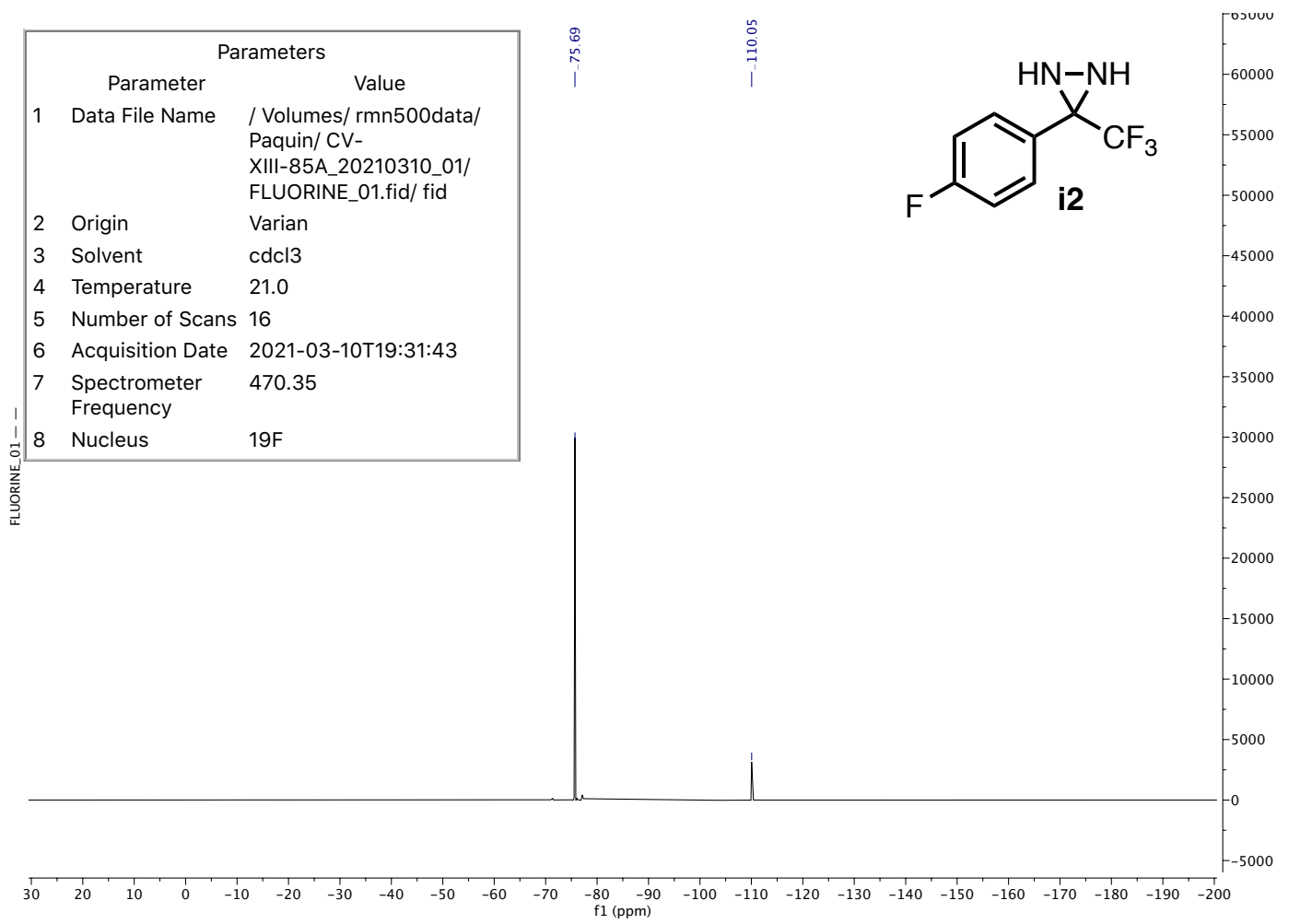

\section{3-(4-Bromophenyl)-3-(trifluoromethyl)diaziridine j2}

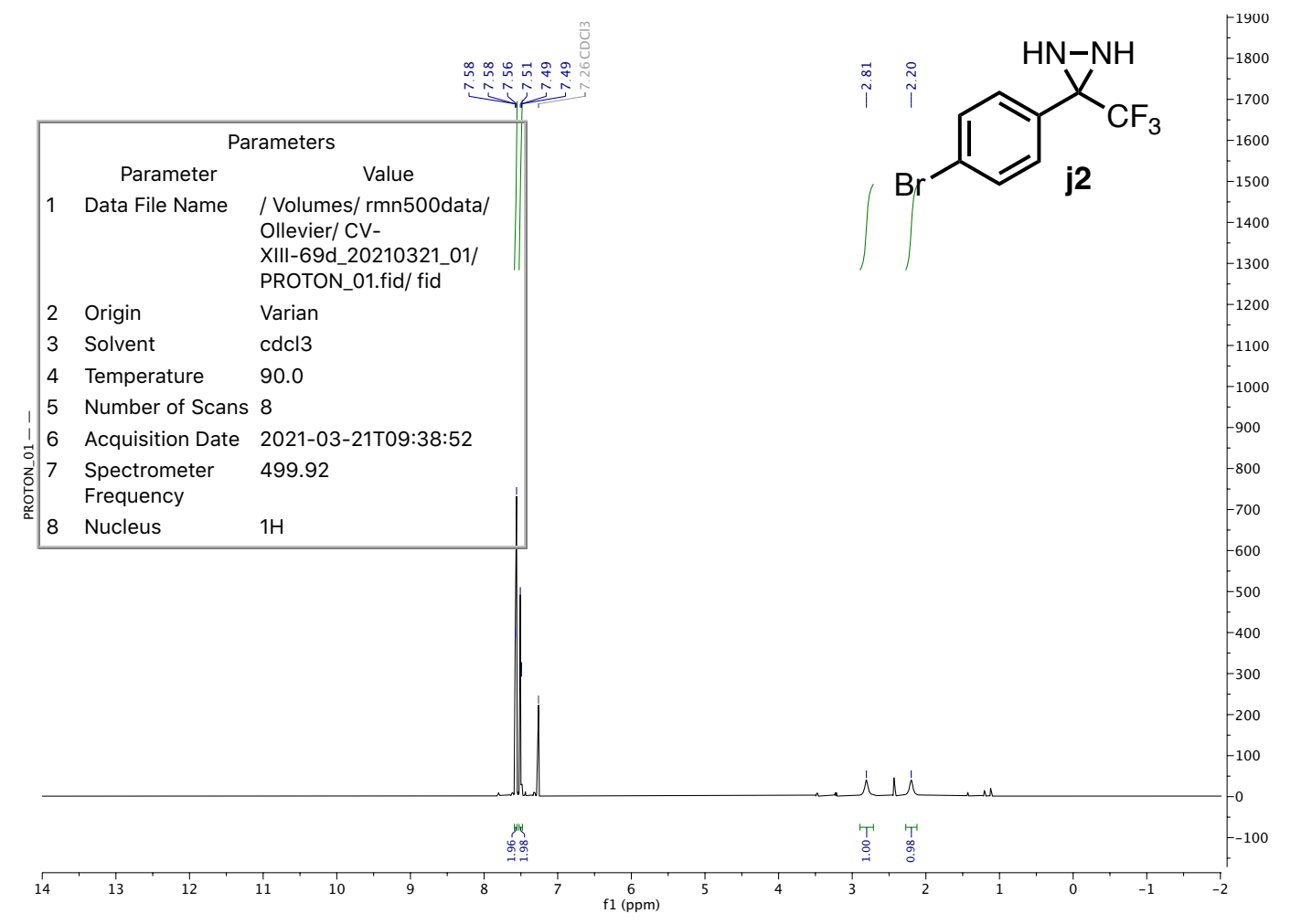




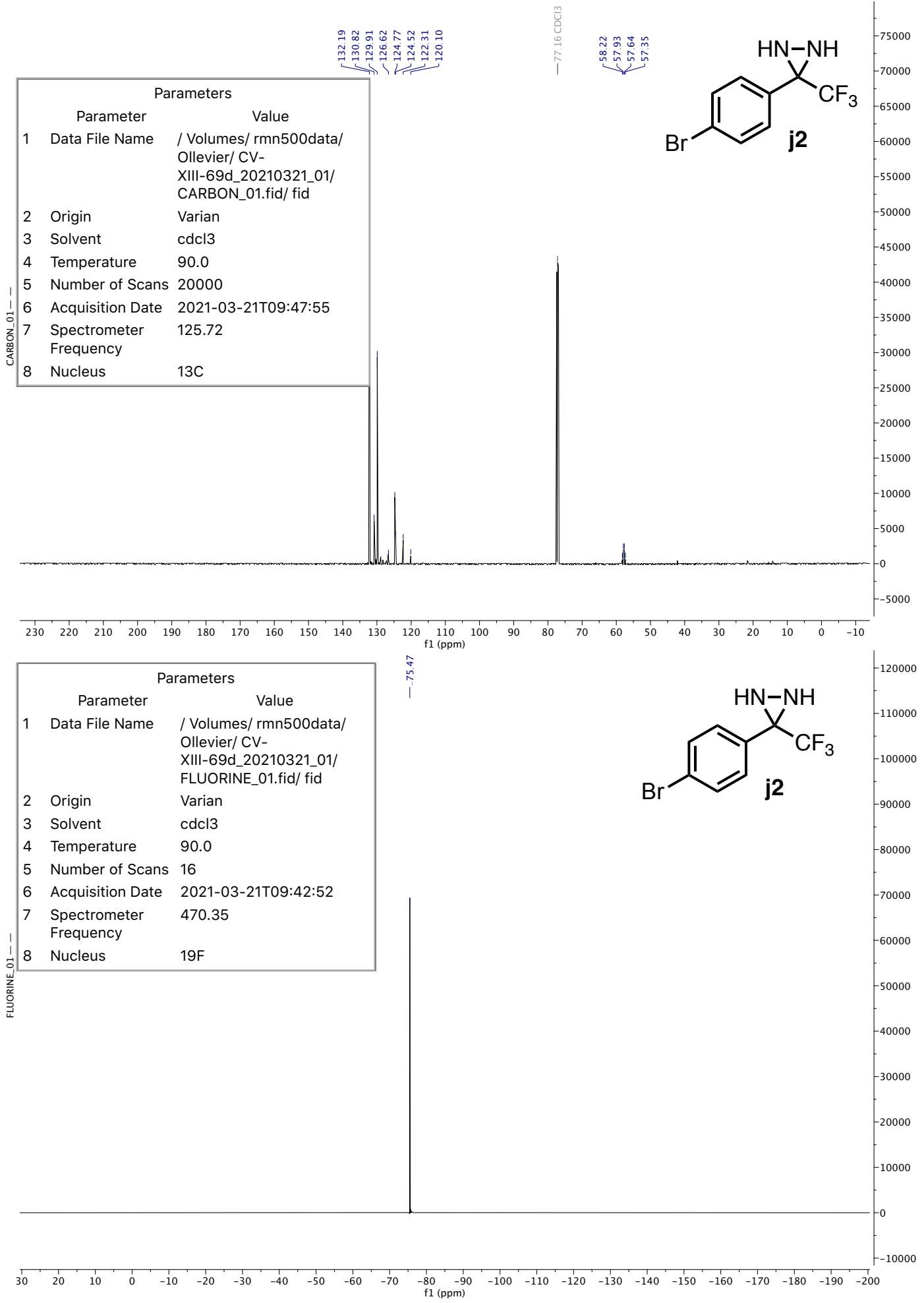




\section{3-(3-Bromophenyl)-3-(trifluoromethyl)diaziridine k2}

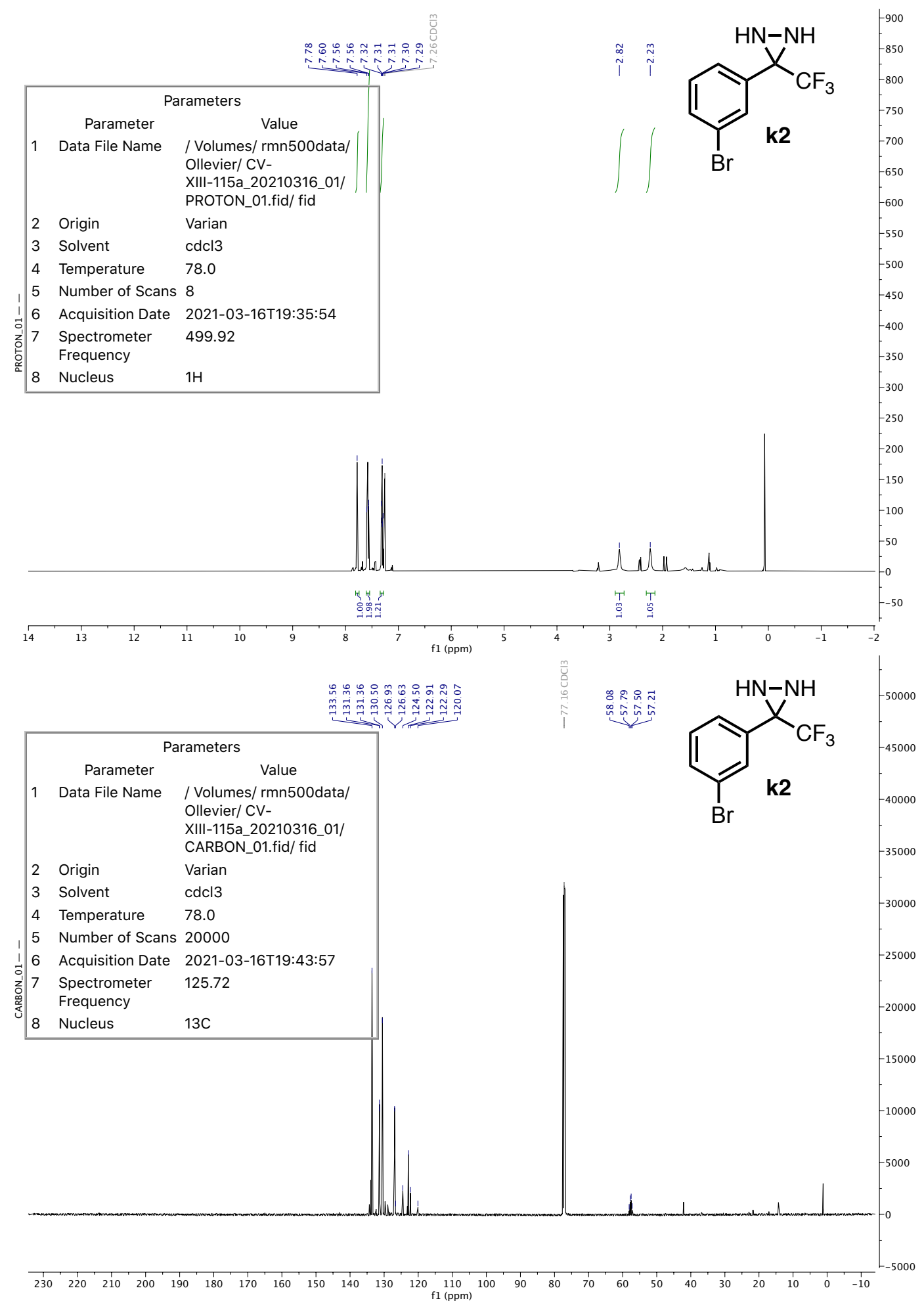




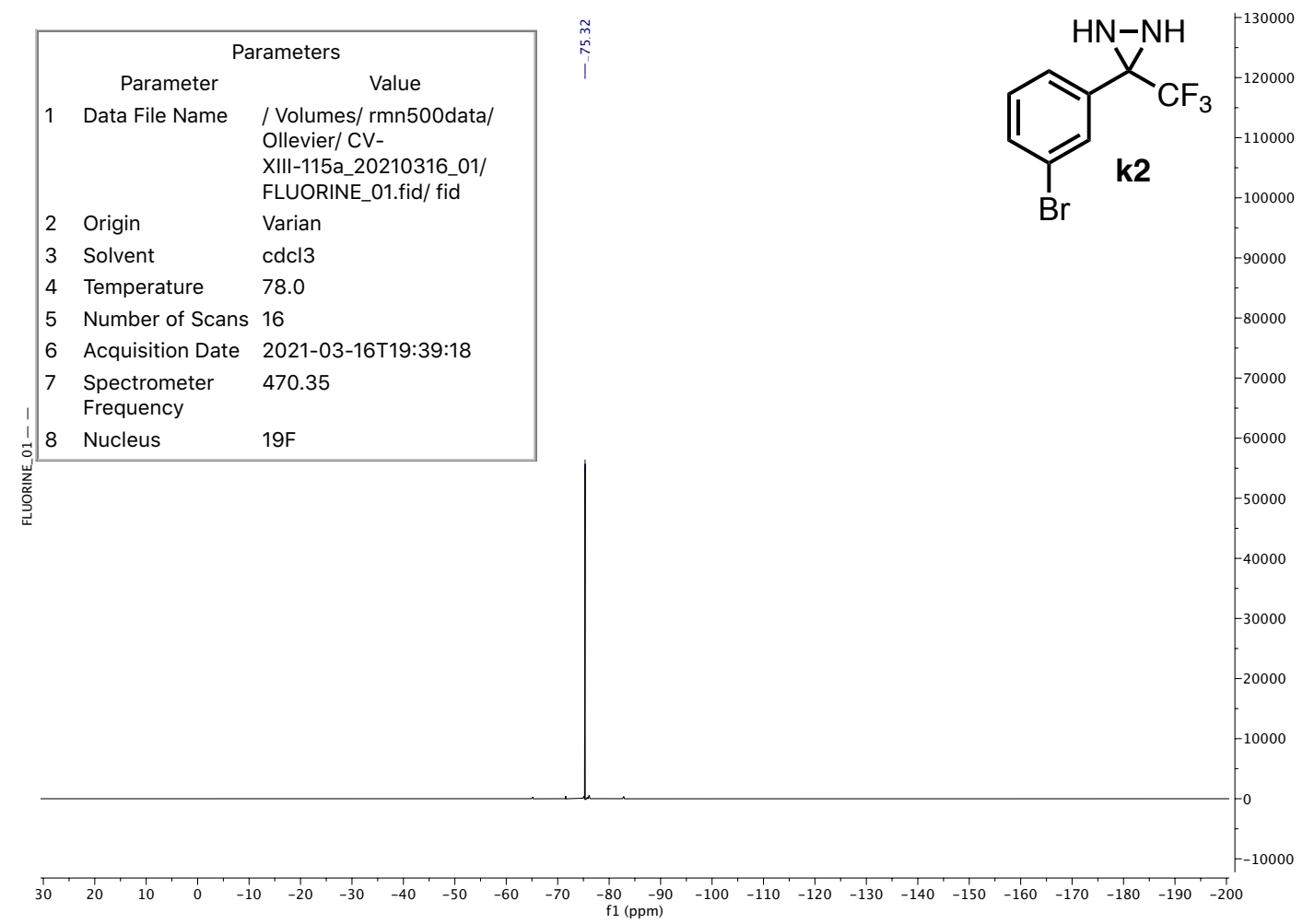

\section{3-(4-Trifluoromethylphenyl)-3-(trifluoromethyl)diaziridine I2}

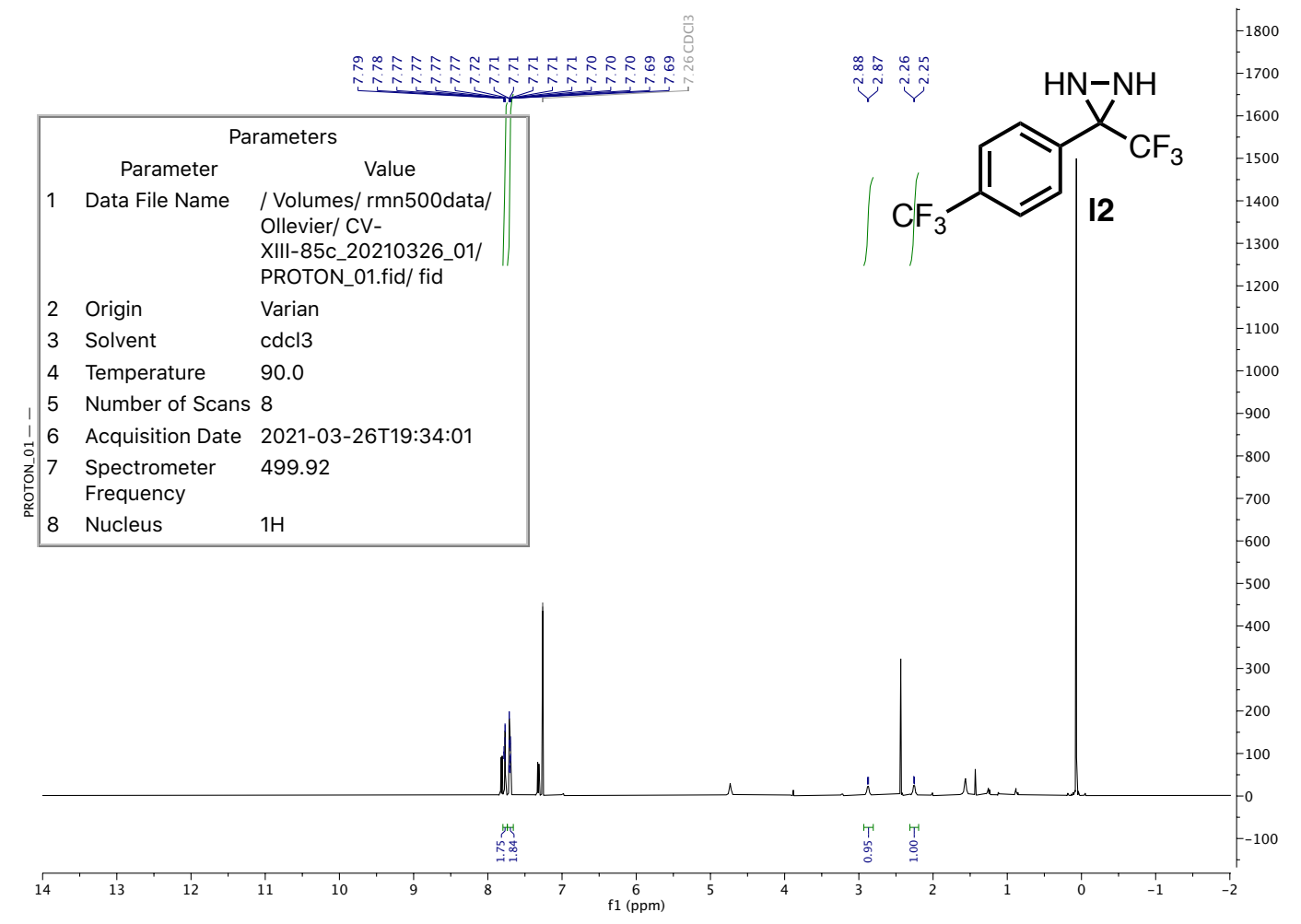




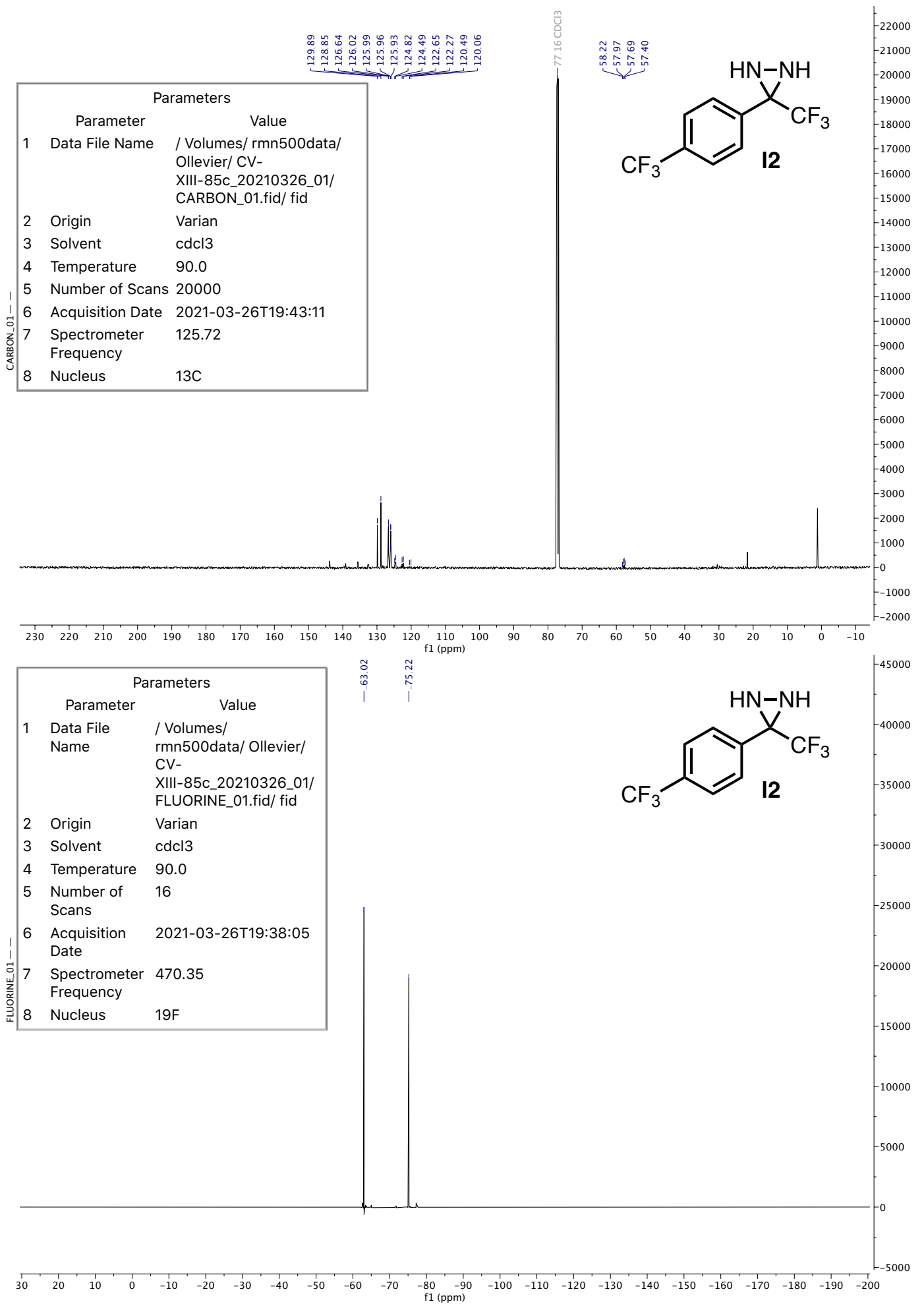




\section{3-(2-Methoxyphenyl)-3-(trifluoromethyl)diaziridine m2}

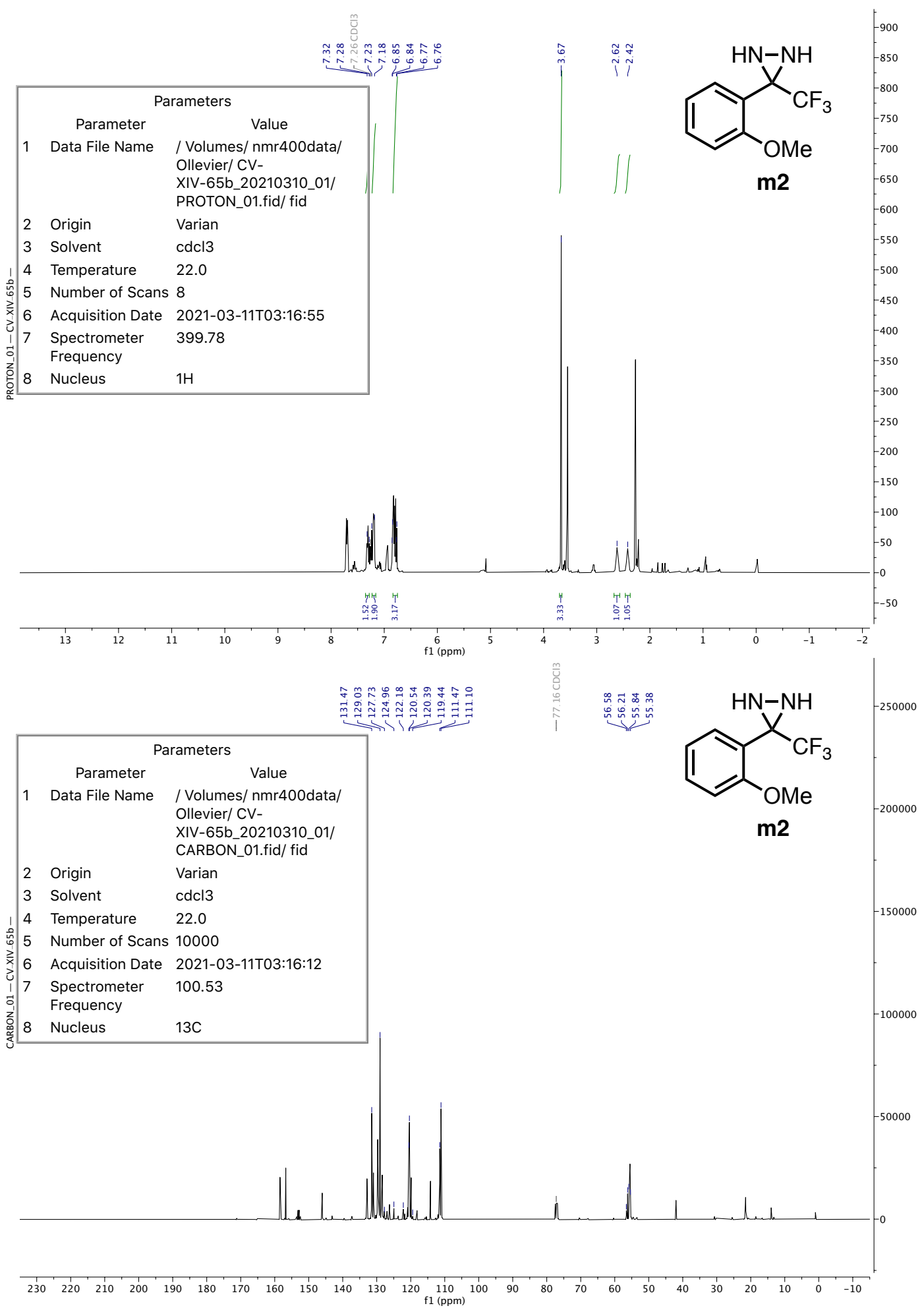




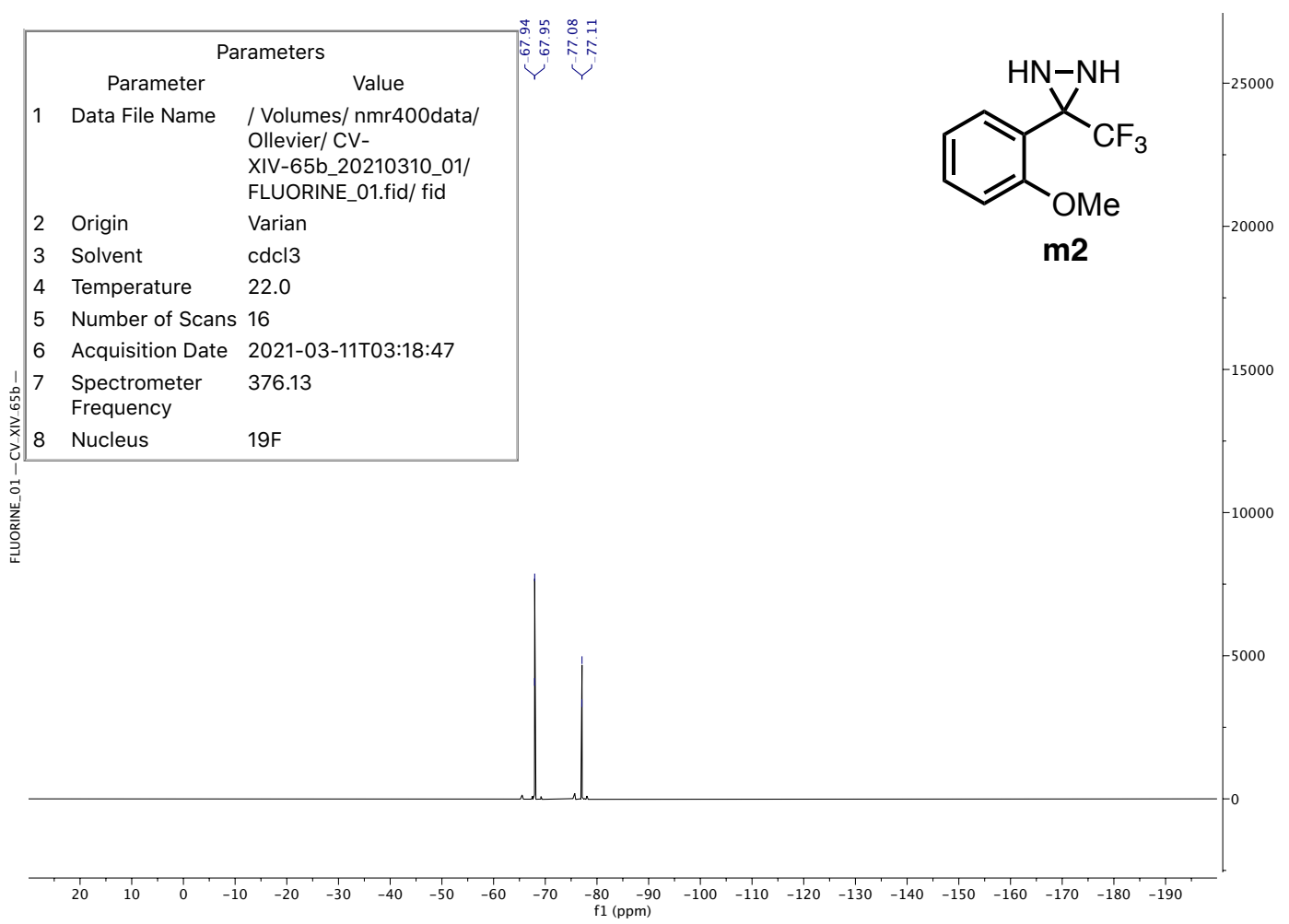

\section{3-(2-Fluorophenyl)-3-(trifluoromethyl)diaziridine n2}

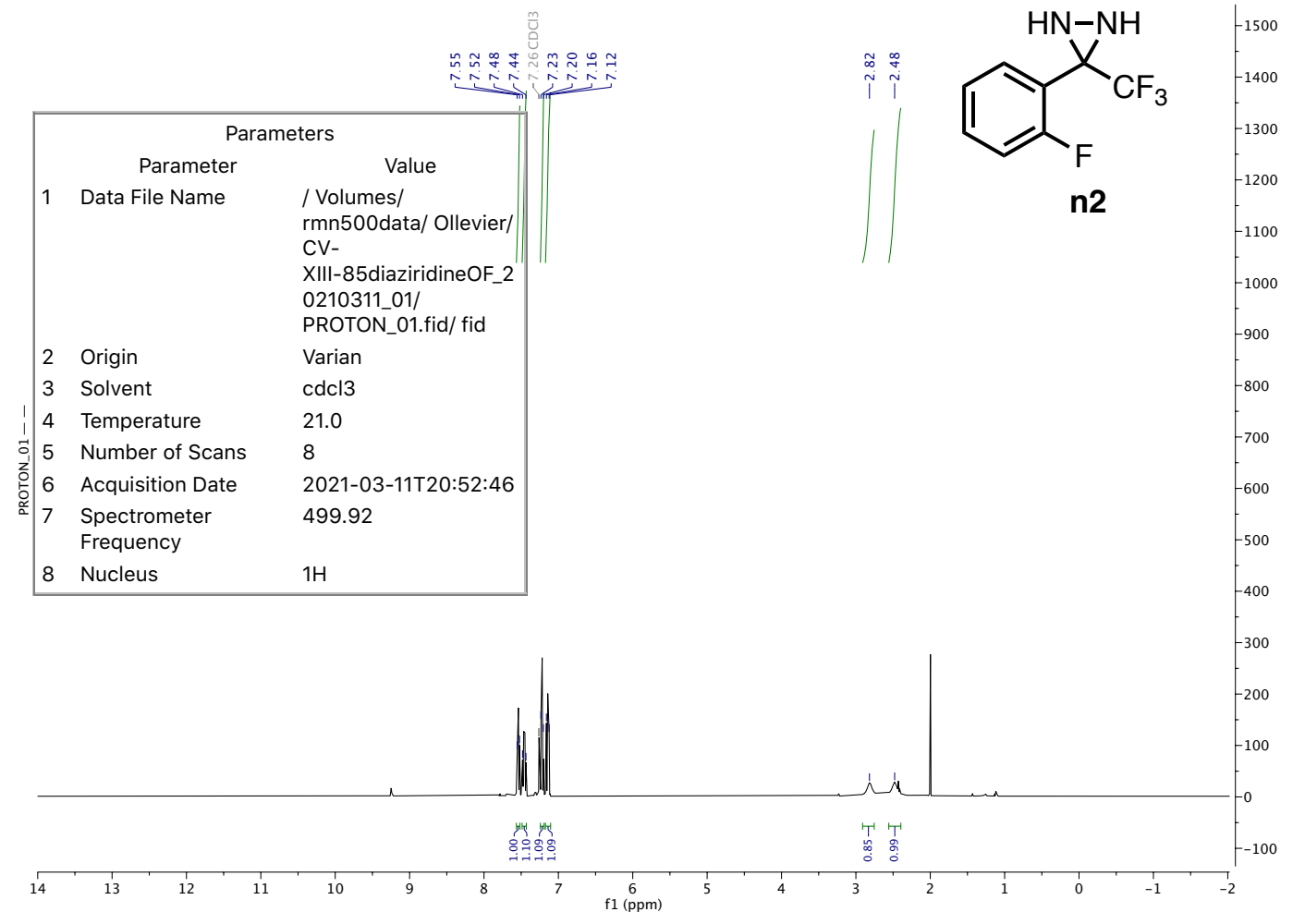




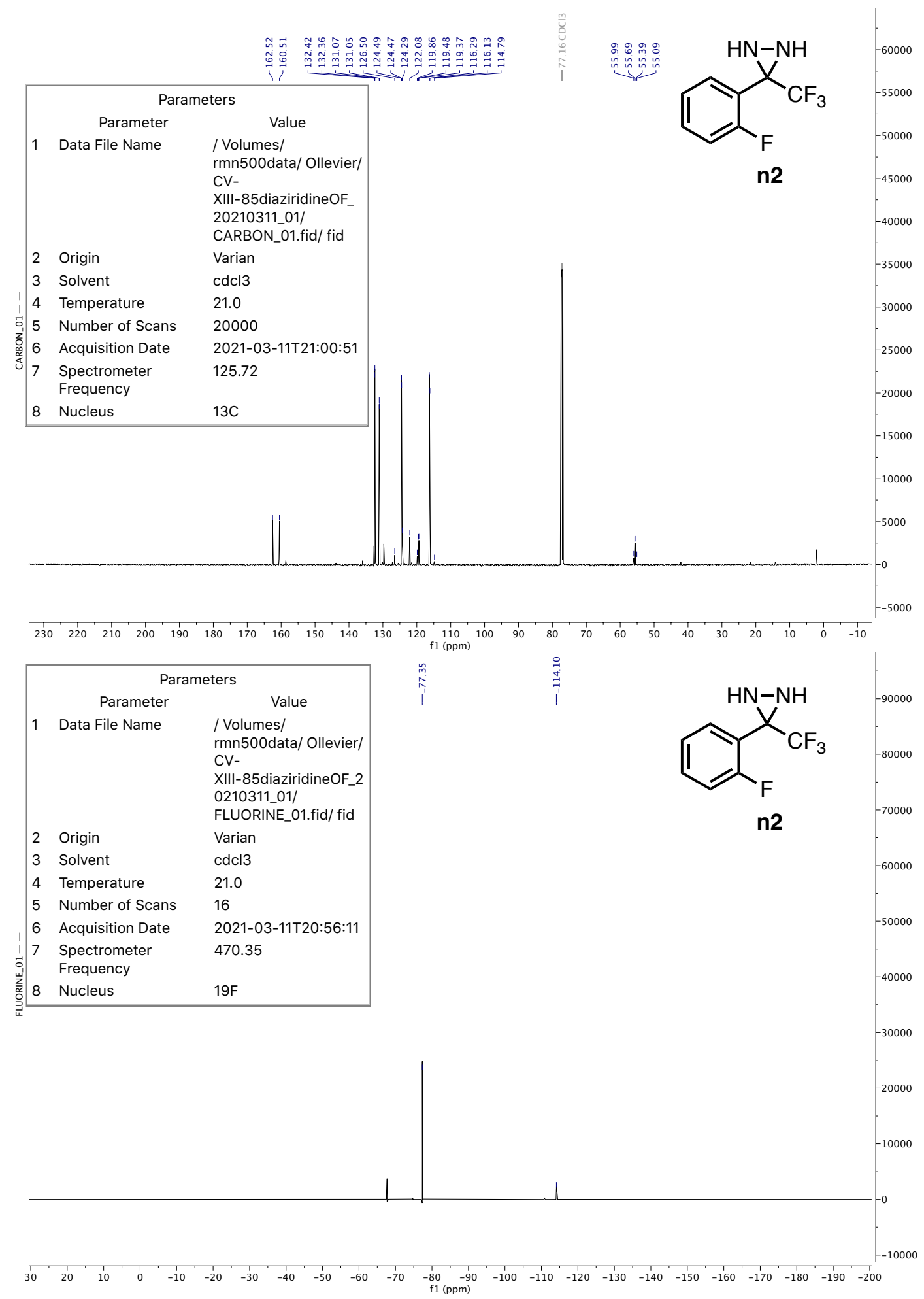




\section{3-([1,1'-Biphenyl]-4-yl)-3-(trifluoromethyl)-3H-diazirine 1a}

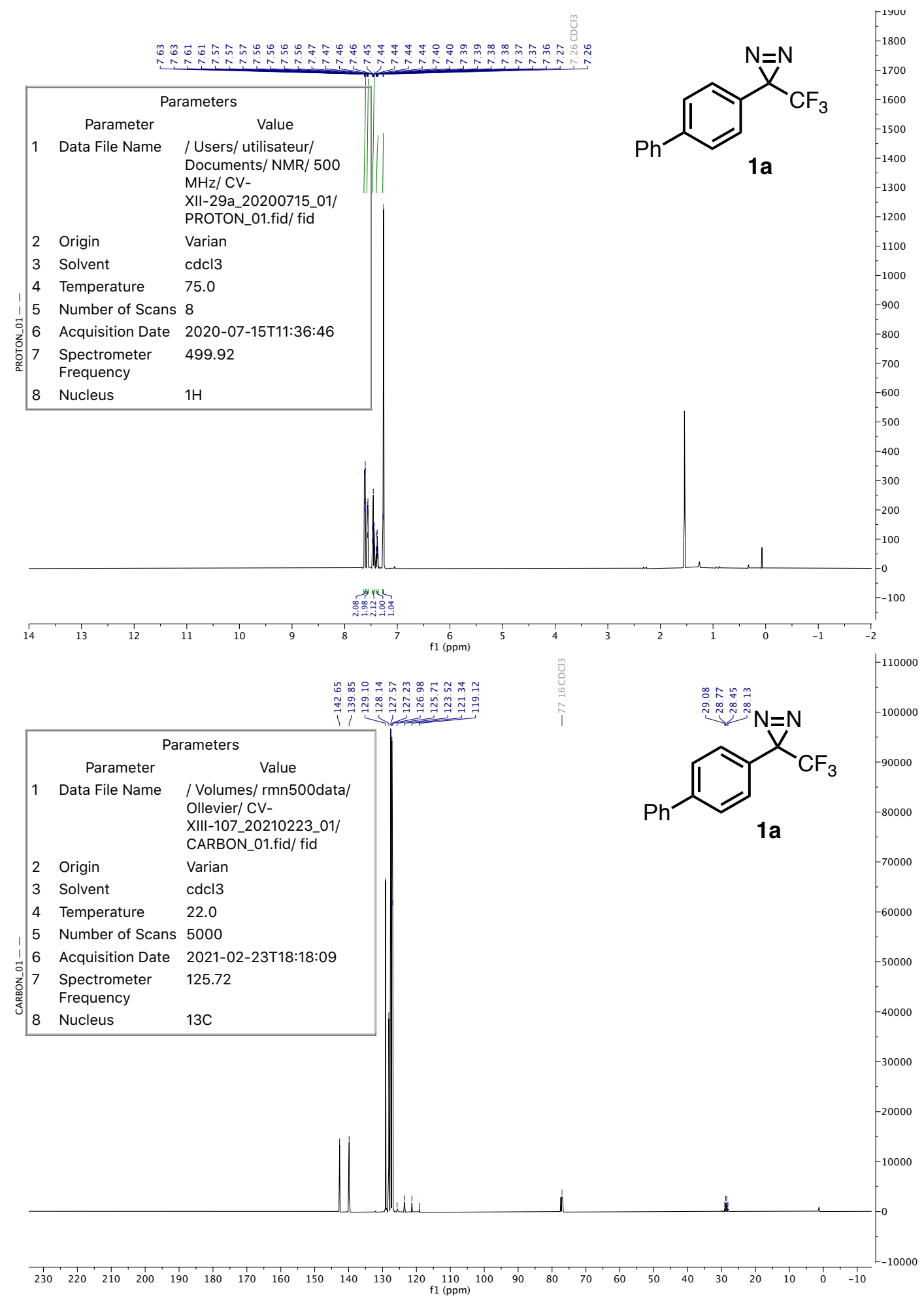




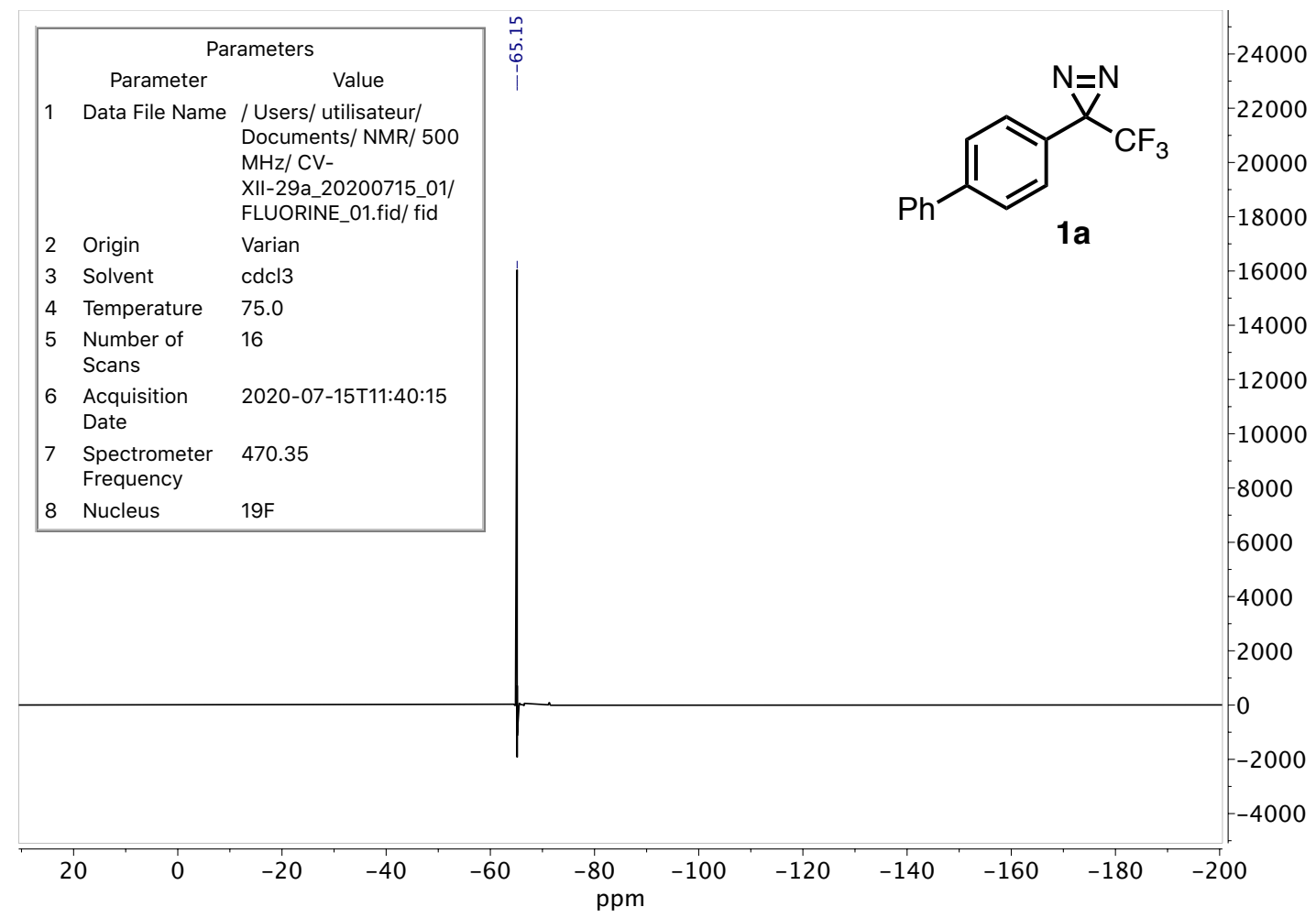

\section{3-(Naphthalen-2-yl)-3-(trifluoromethyl)-3H-diazirine 1b}

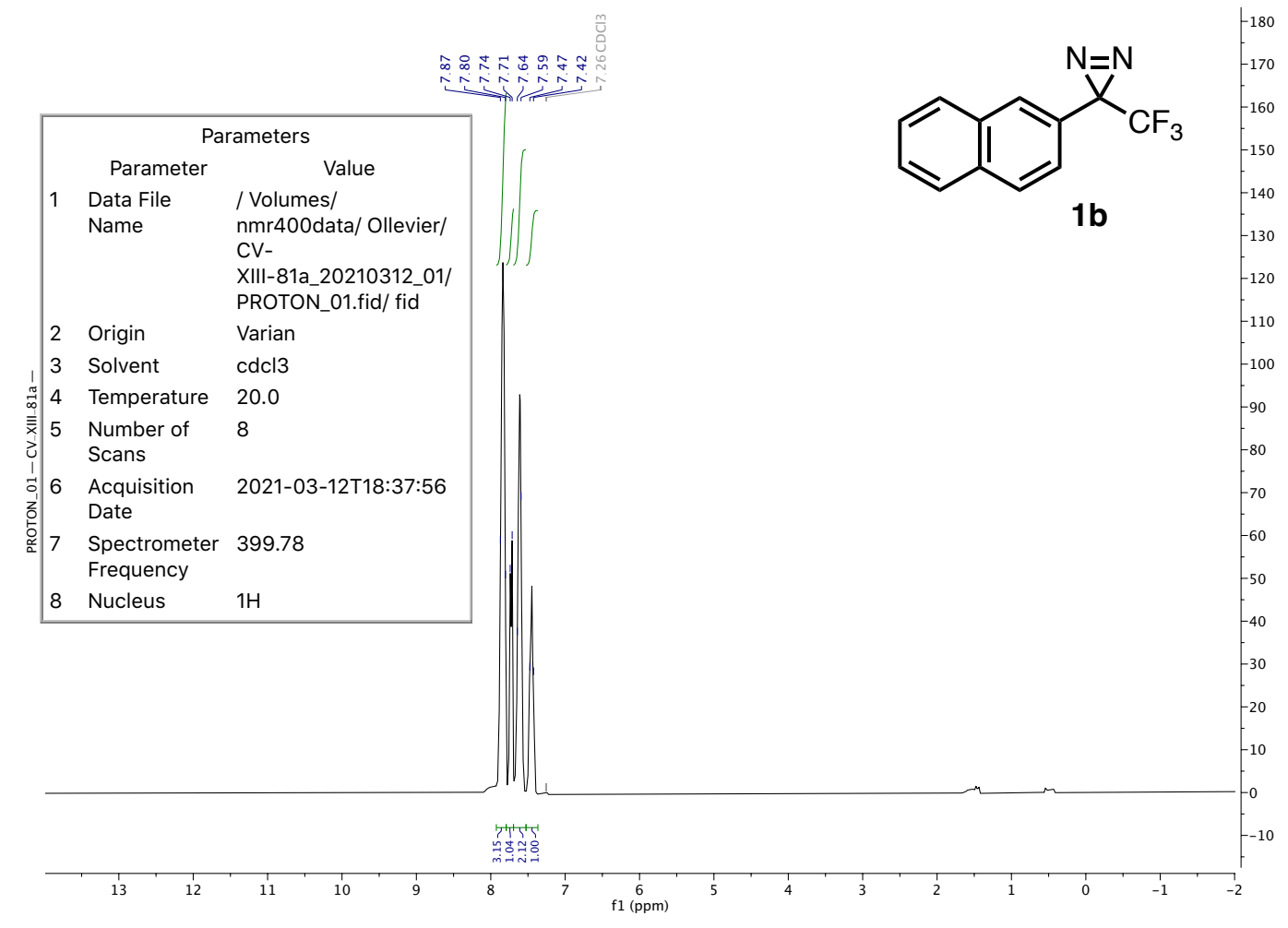




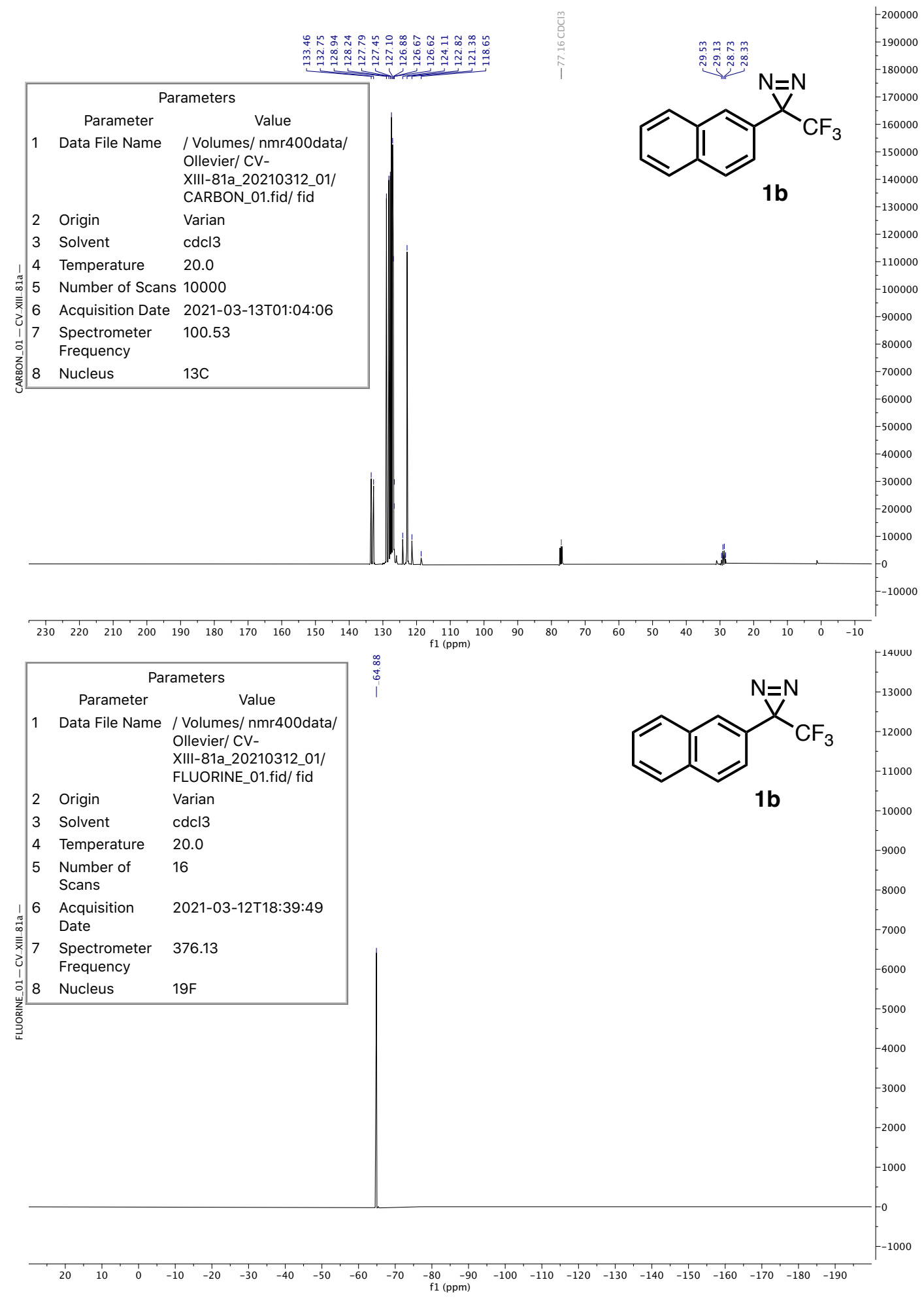


3-(4-(Benzyloxy)phenyl)-3-(trifluoromethyl)-3H-diazirine 1c

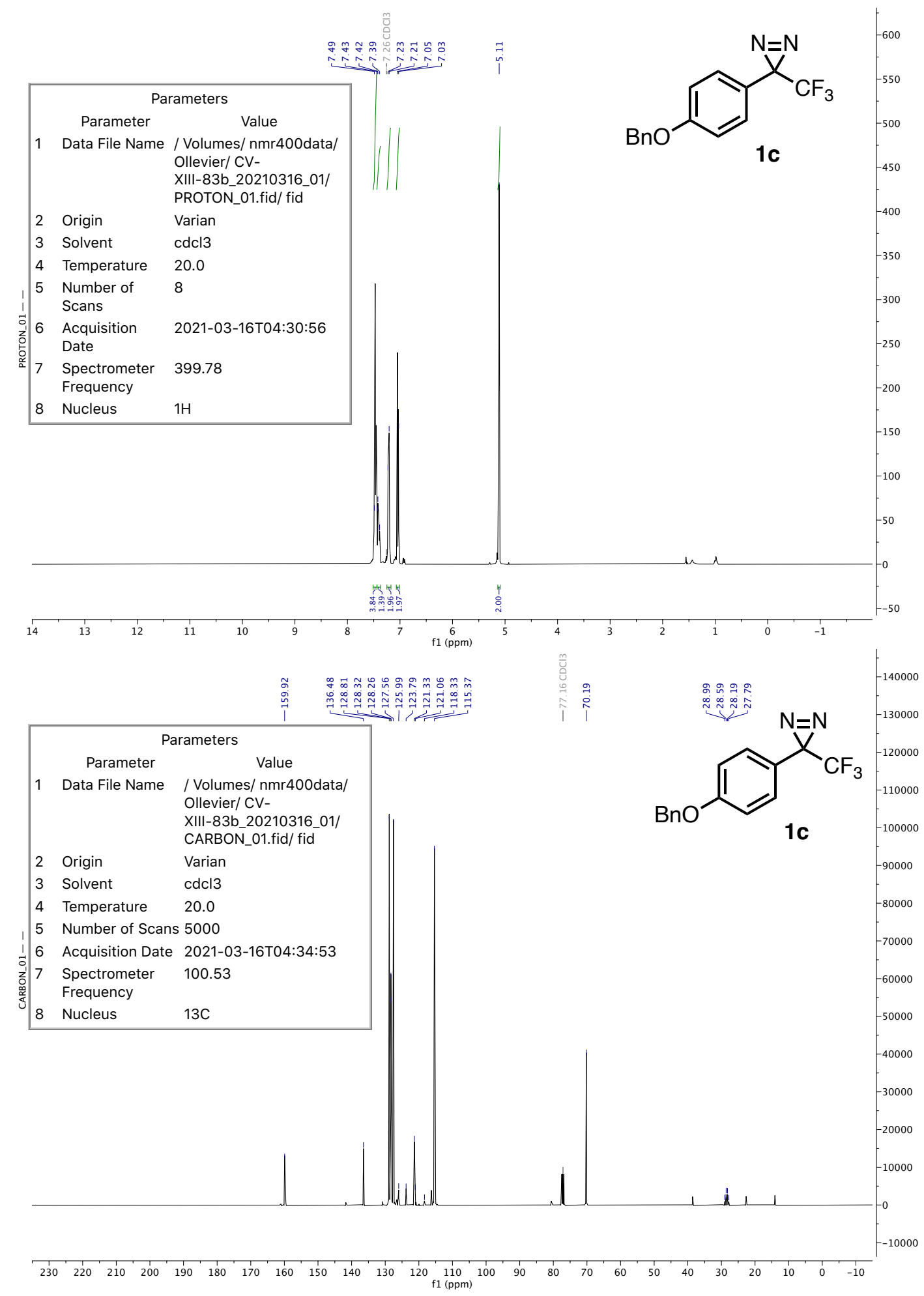




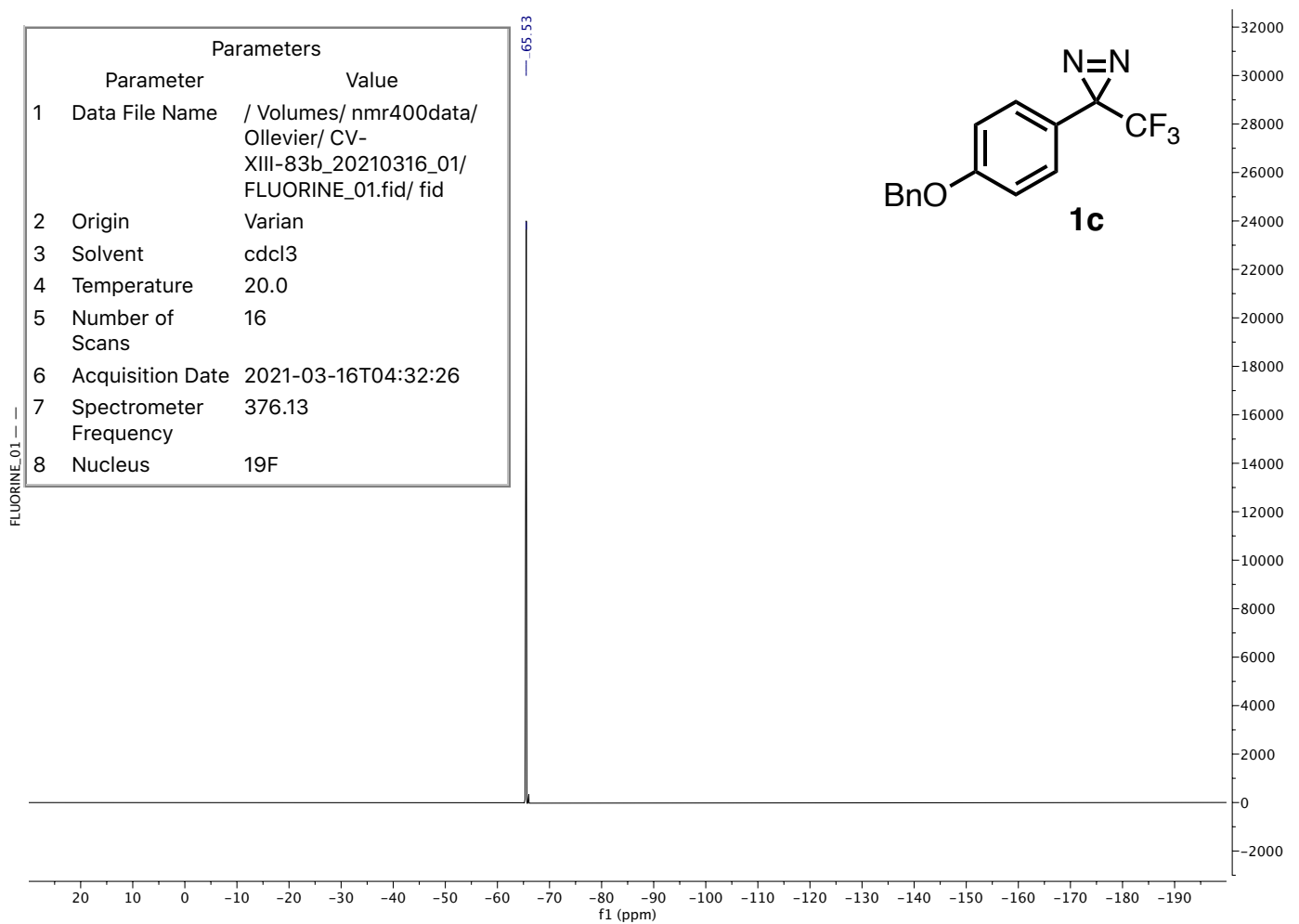

\section{4,4'-Bis(3-(trifluoromethyl)-3H-diazirin-3-yl)-1,1'-biphenyl 1d}

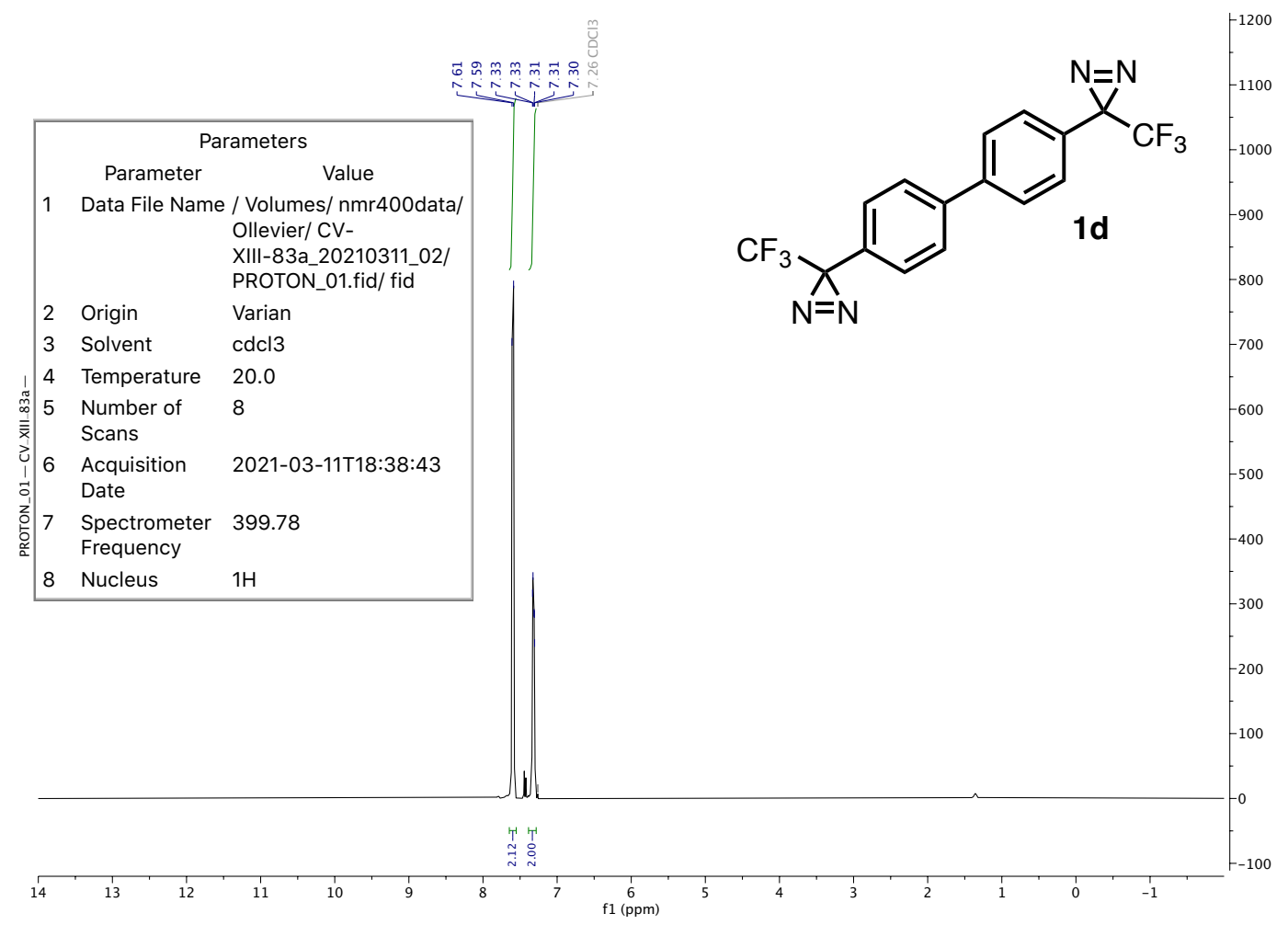




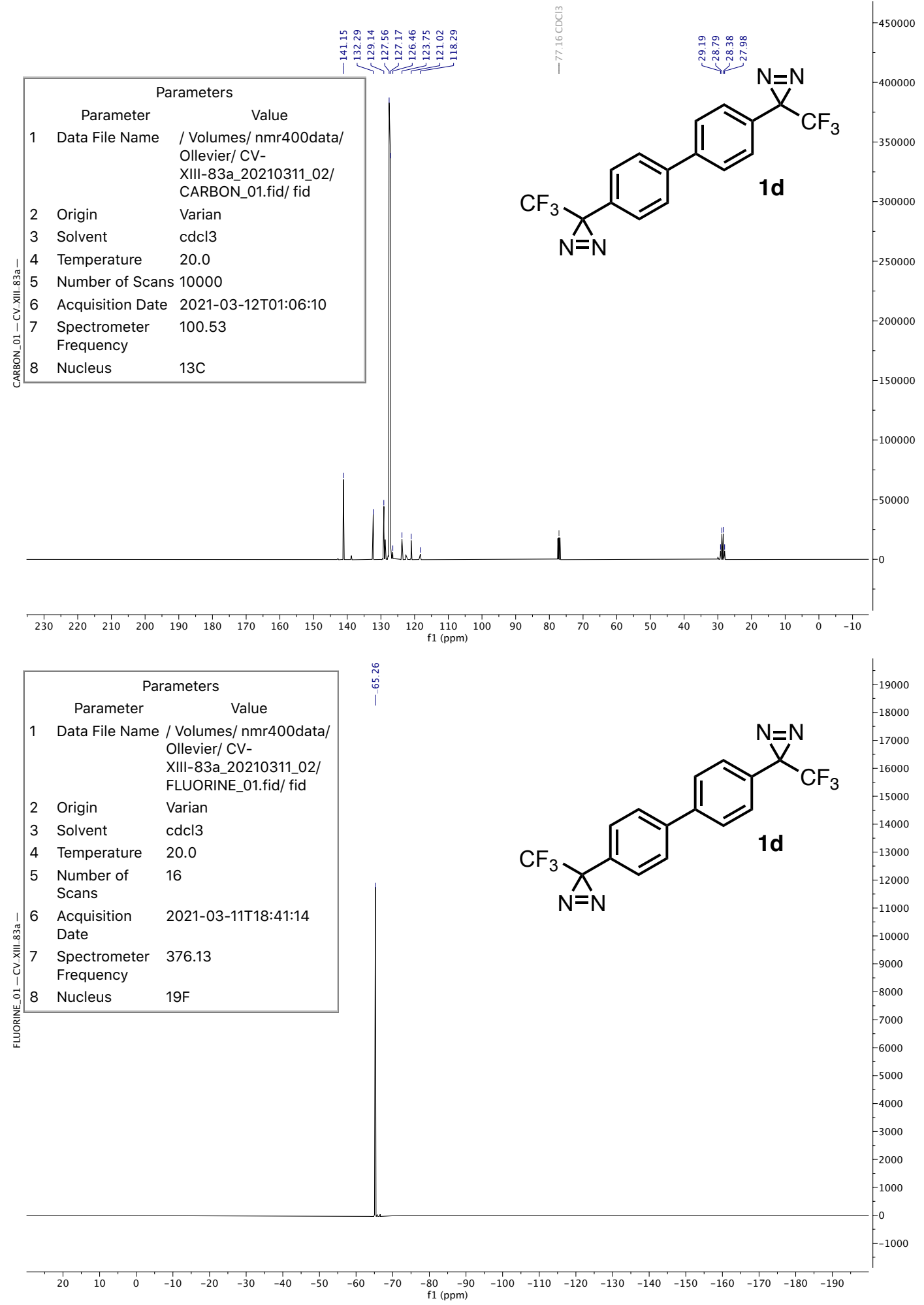


3-(p-Tolyl)-3-(trifluoromethyl)-3H-diazirine $1 \mathrm{e}$

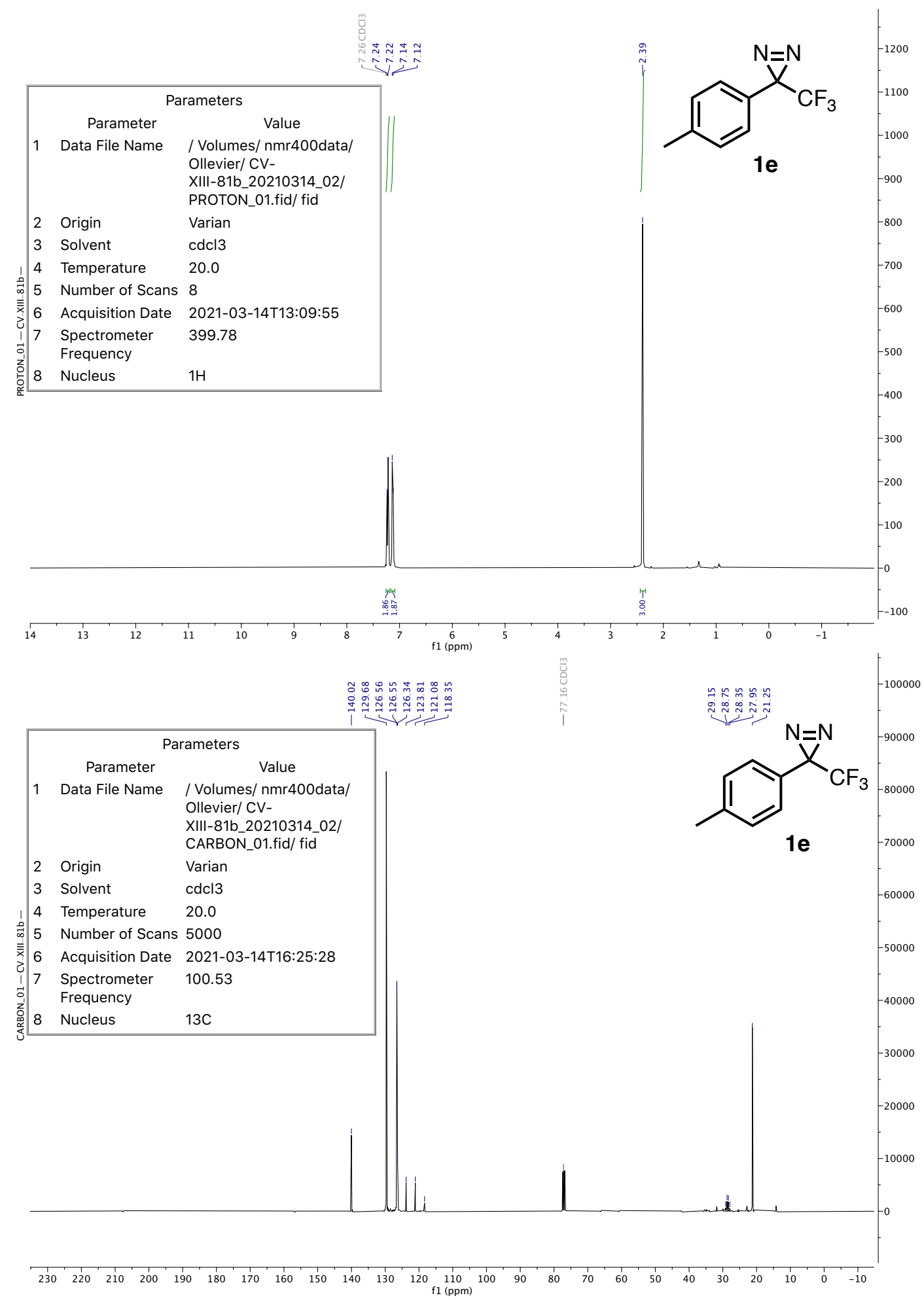




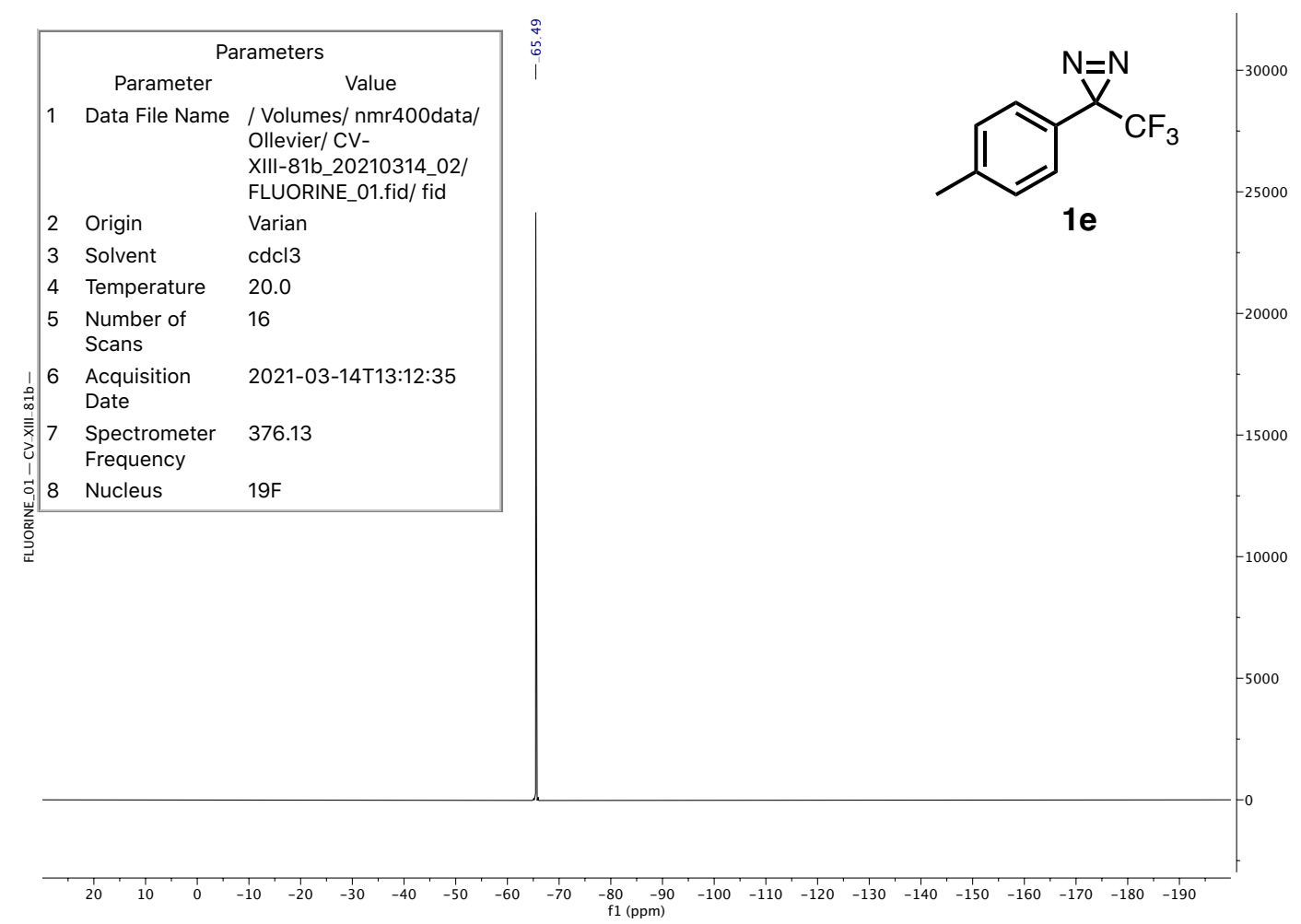

\section{3-(4-(Tert-butyl)phenyl)-3-(trifluoromethyl)-3H-diazirine $1 f$}

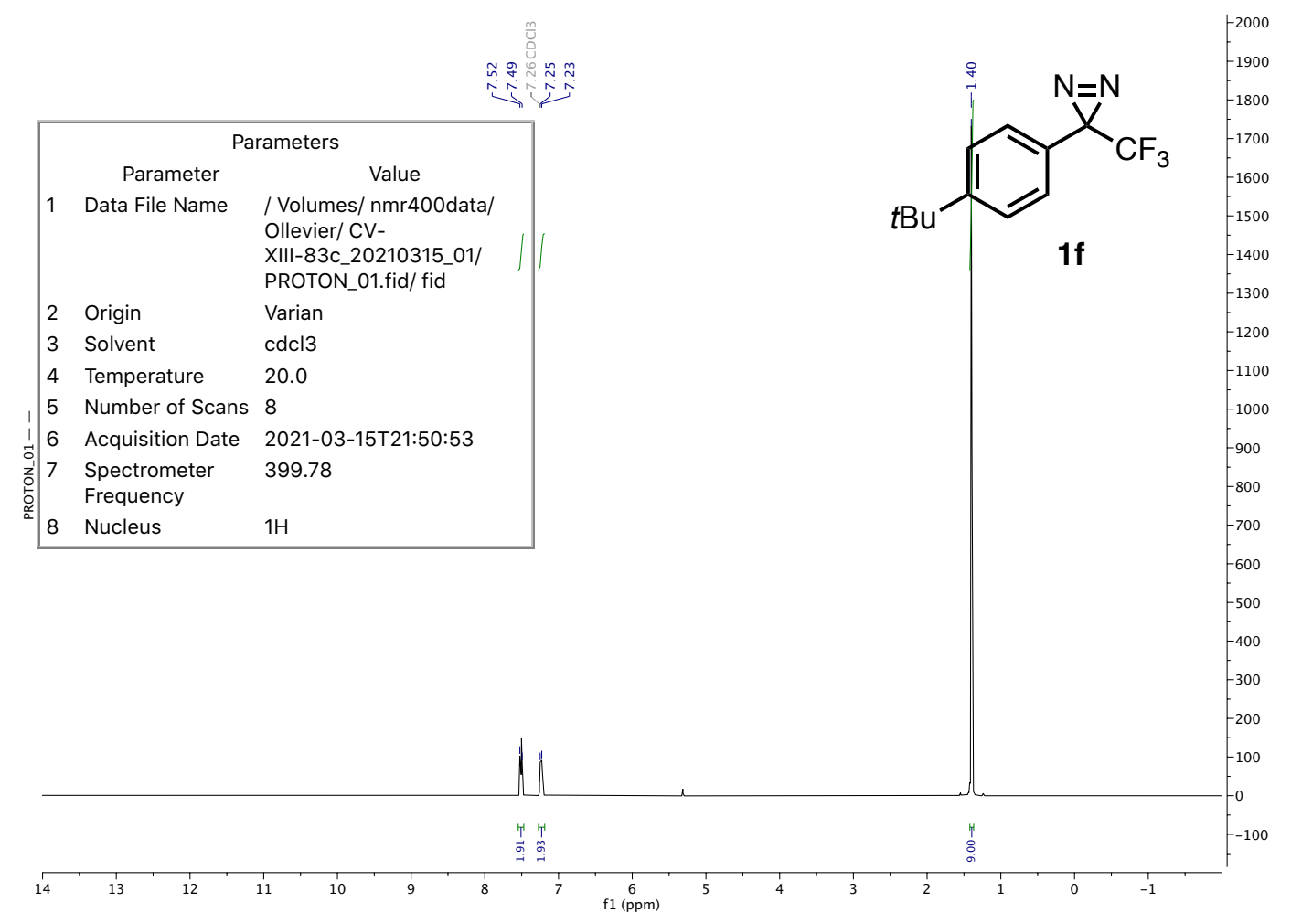




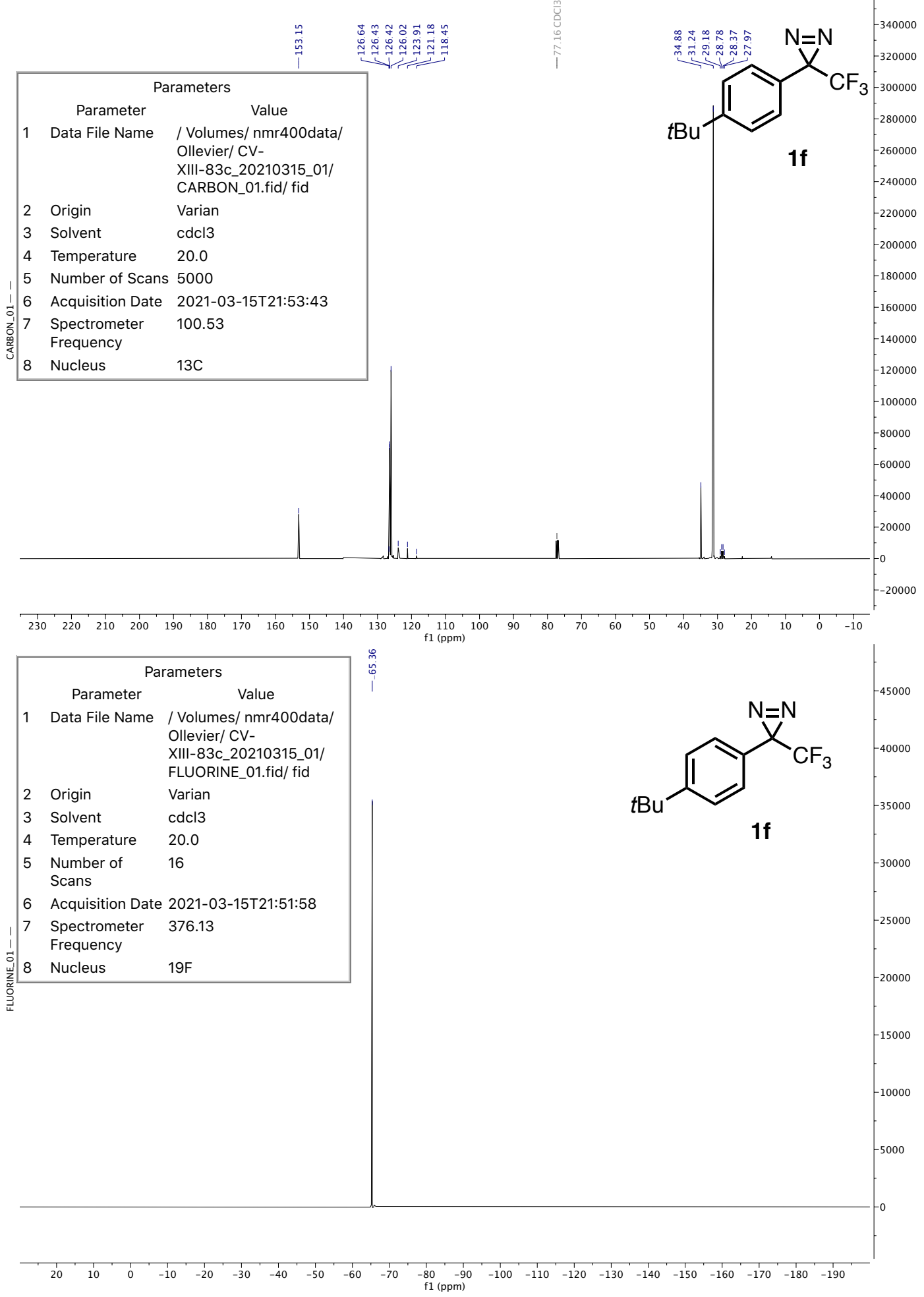


3-(4-Methoxyphenyl)-3-(trifluoromethyl)-3H-diazirine $1 \mathrm{~g}$

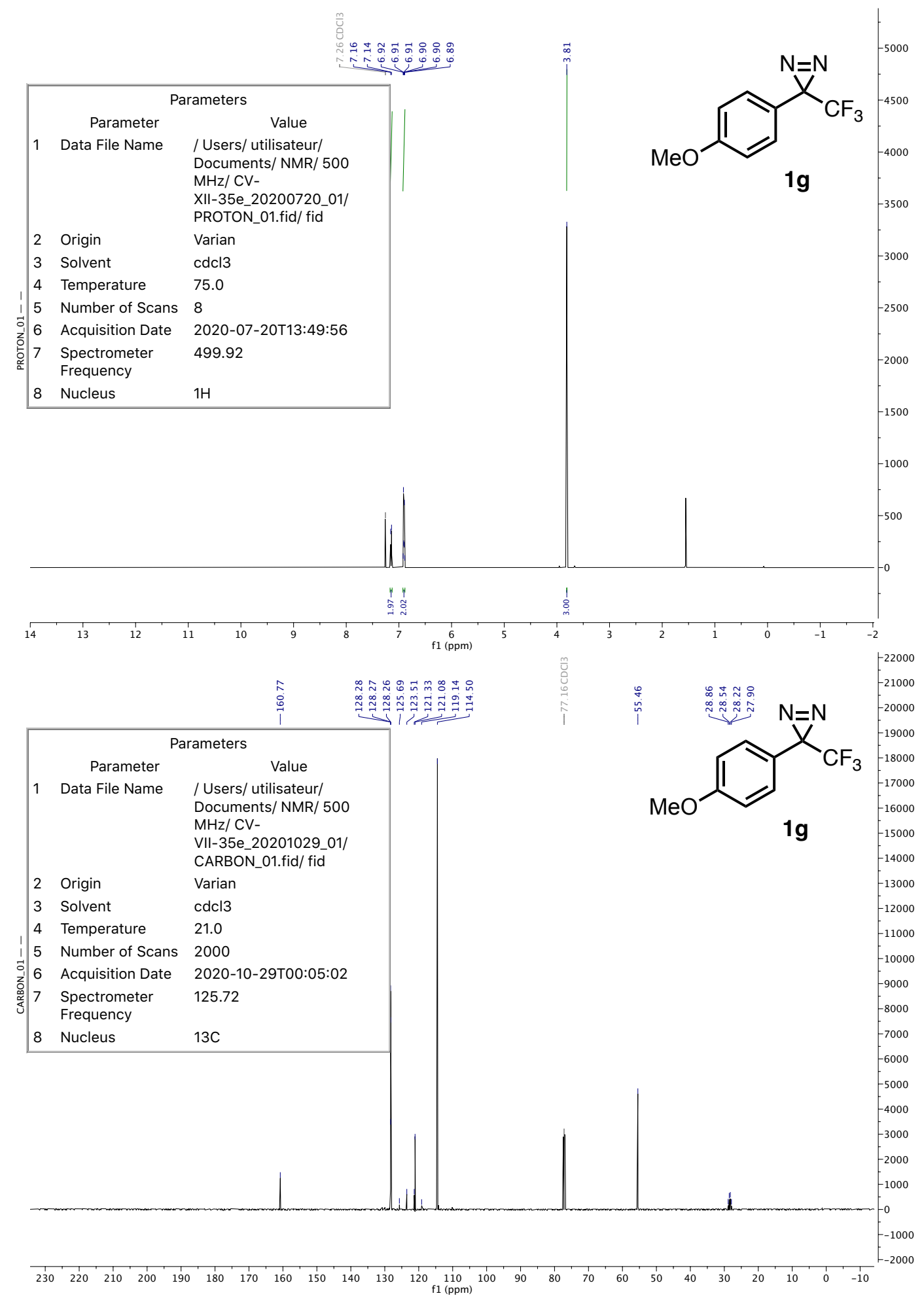




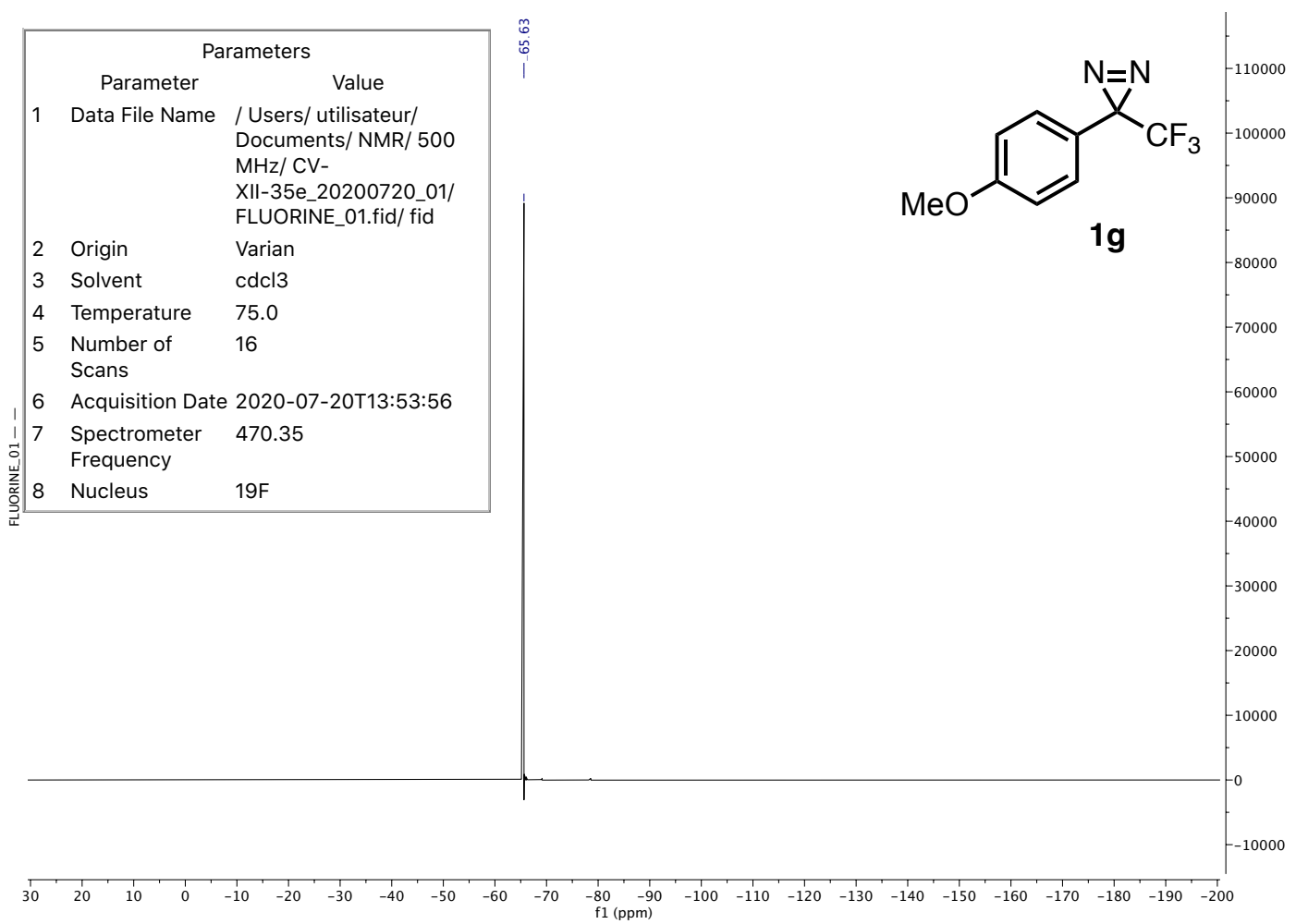

3-Phenyl-3-(trifluoromethyl)-3H-diazirine $1 \mathrm{~h}$

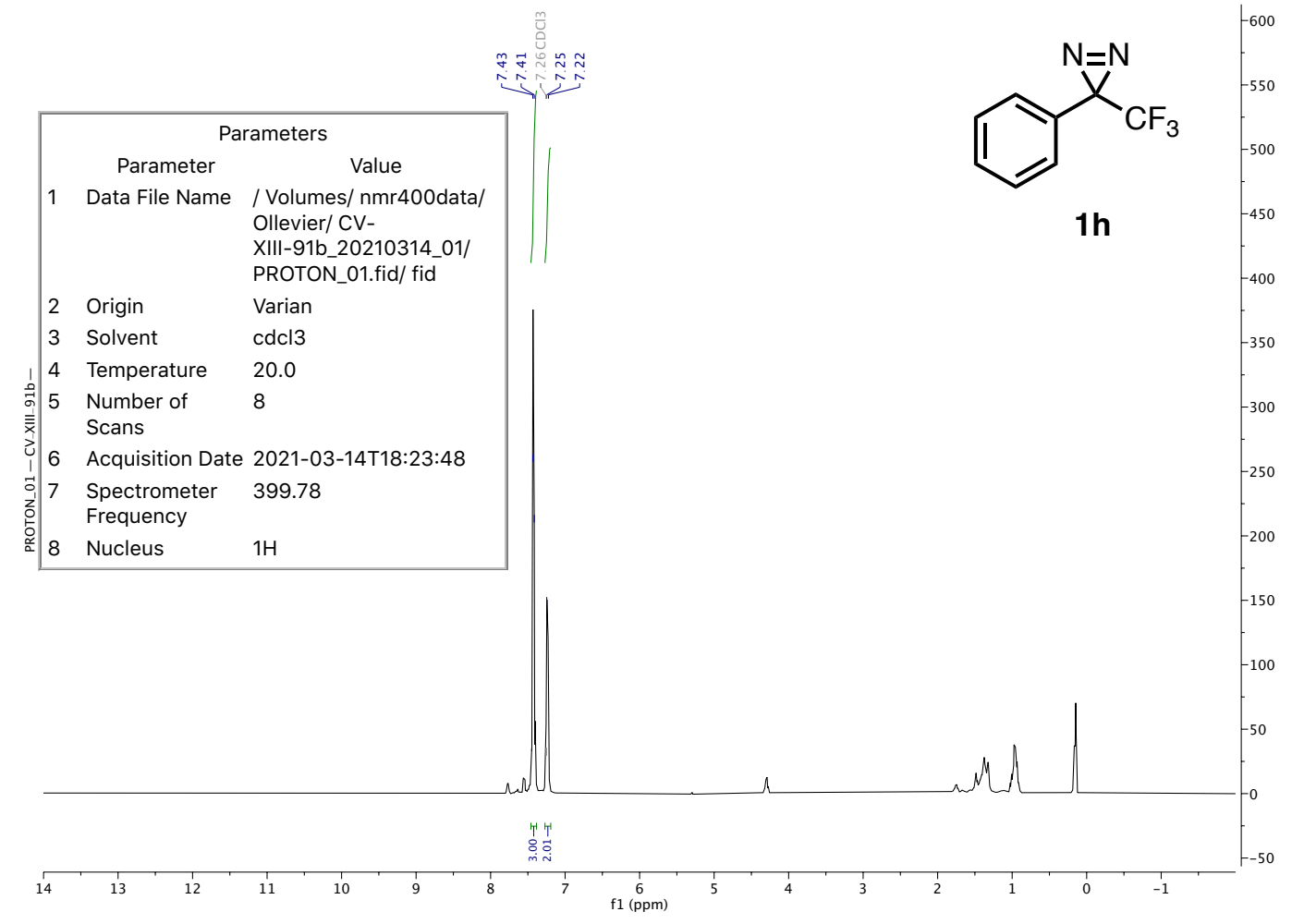




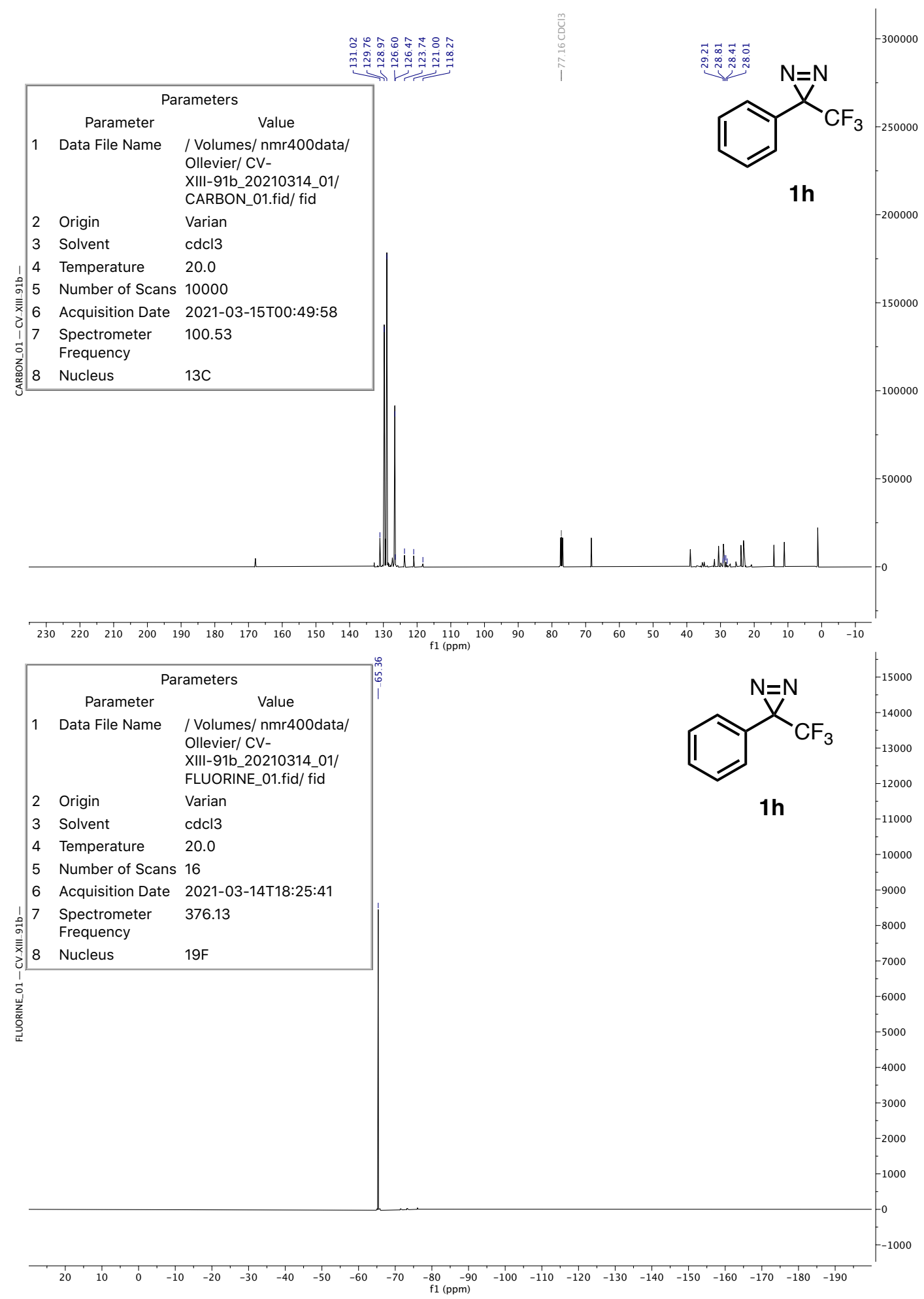


3-(4-Fluorophenyl)-3-(trifluoromethyl)-3H-diazirine 1i

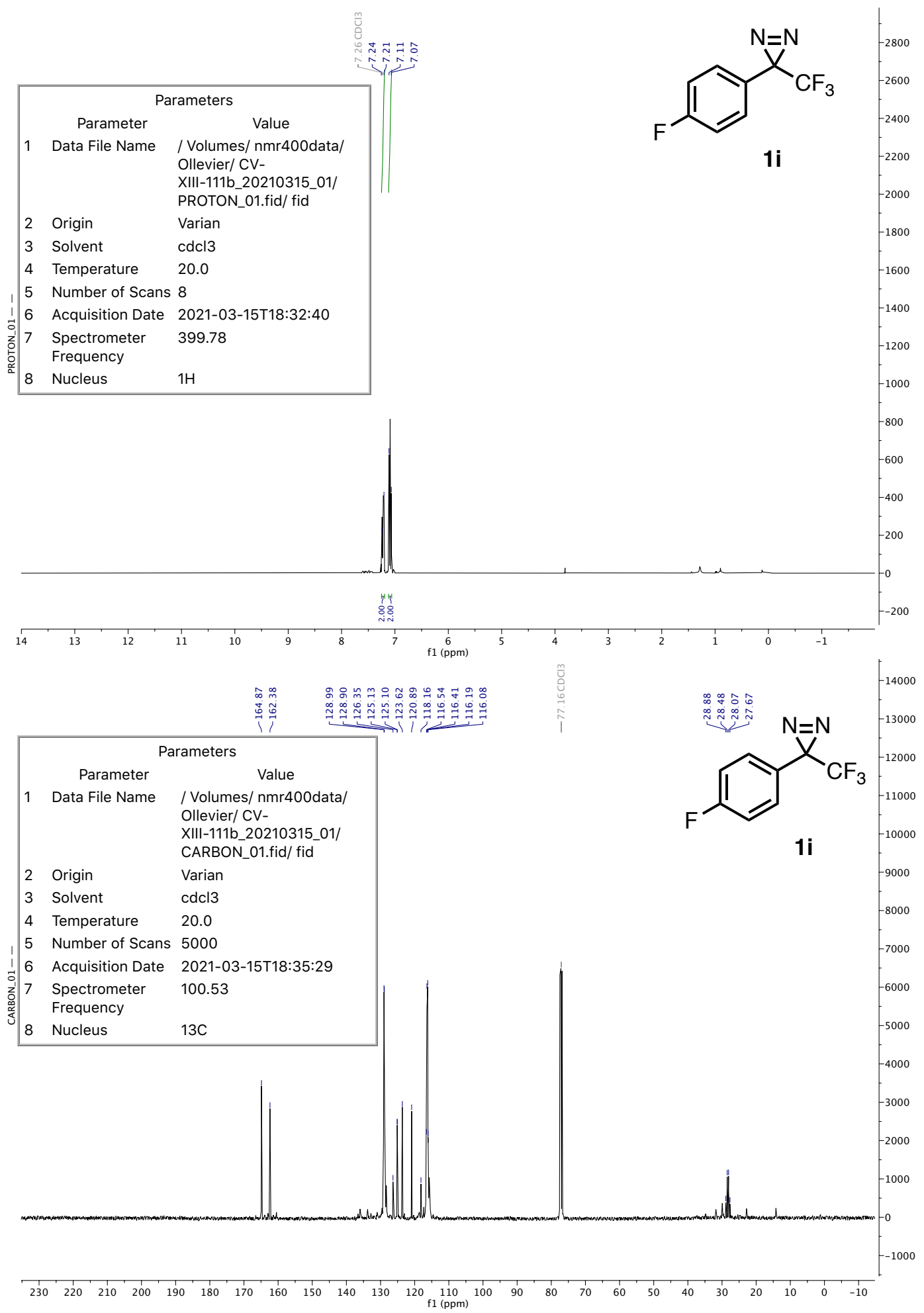




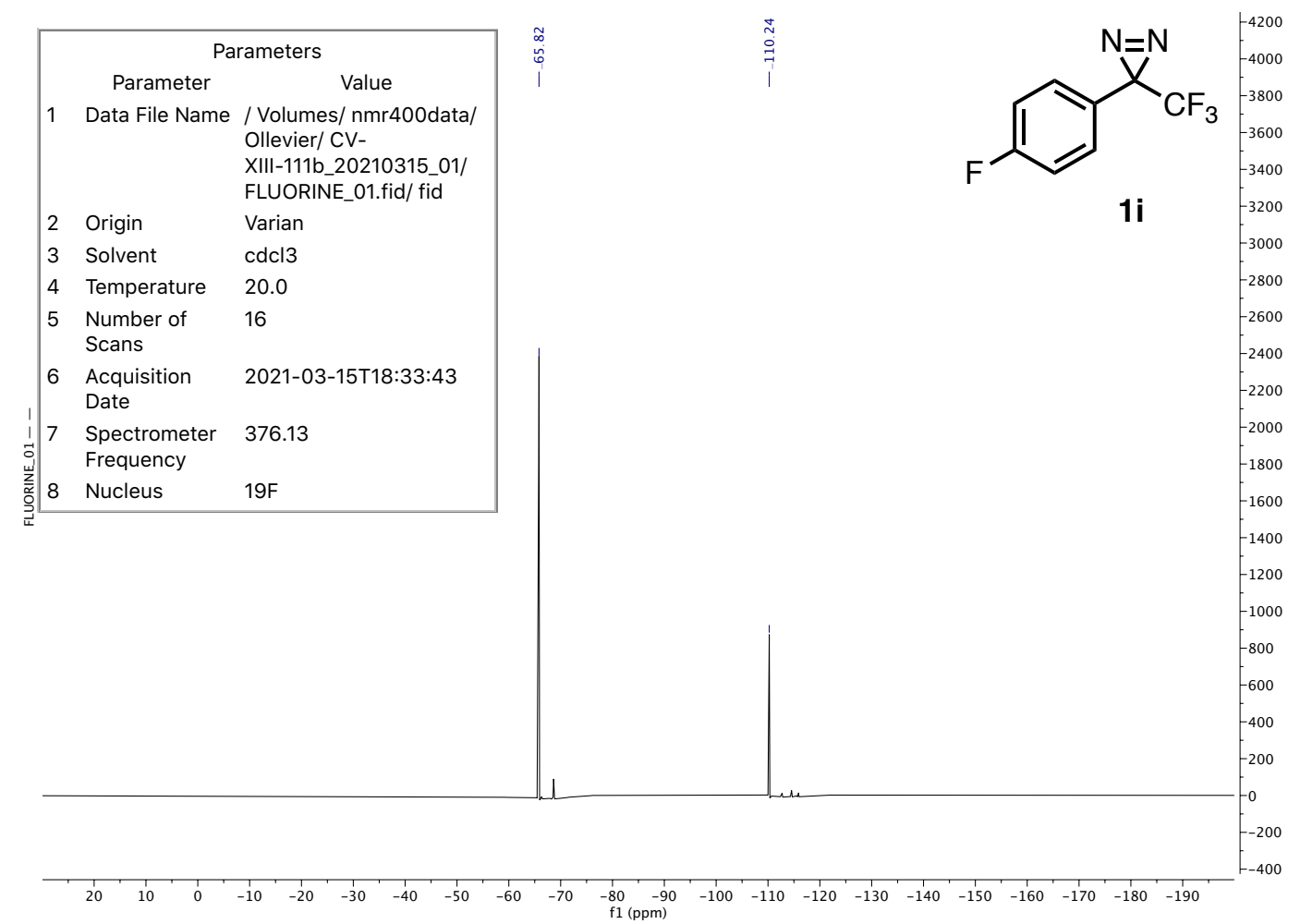

\section{3-(4-Bromophenyl)-3-(trifluoromethyl)-3H-diazirine 1j}

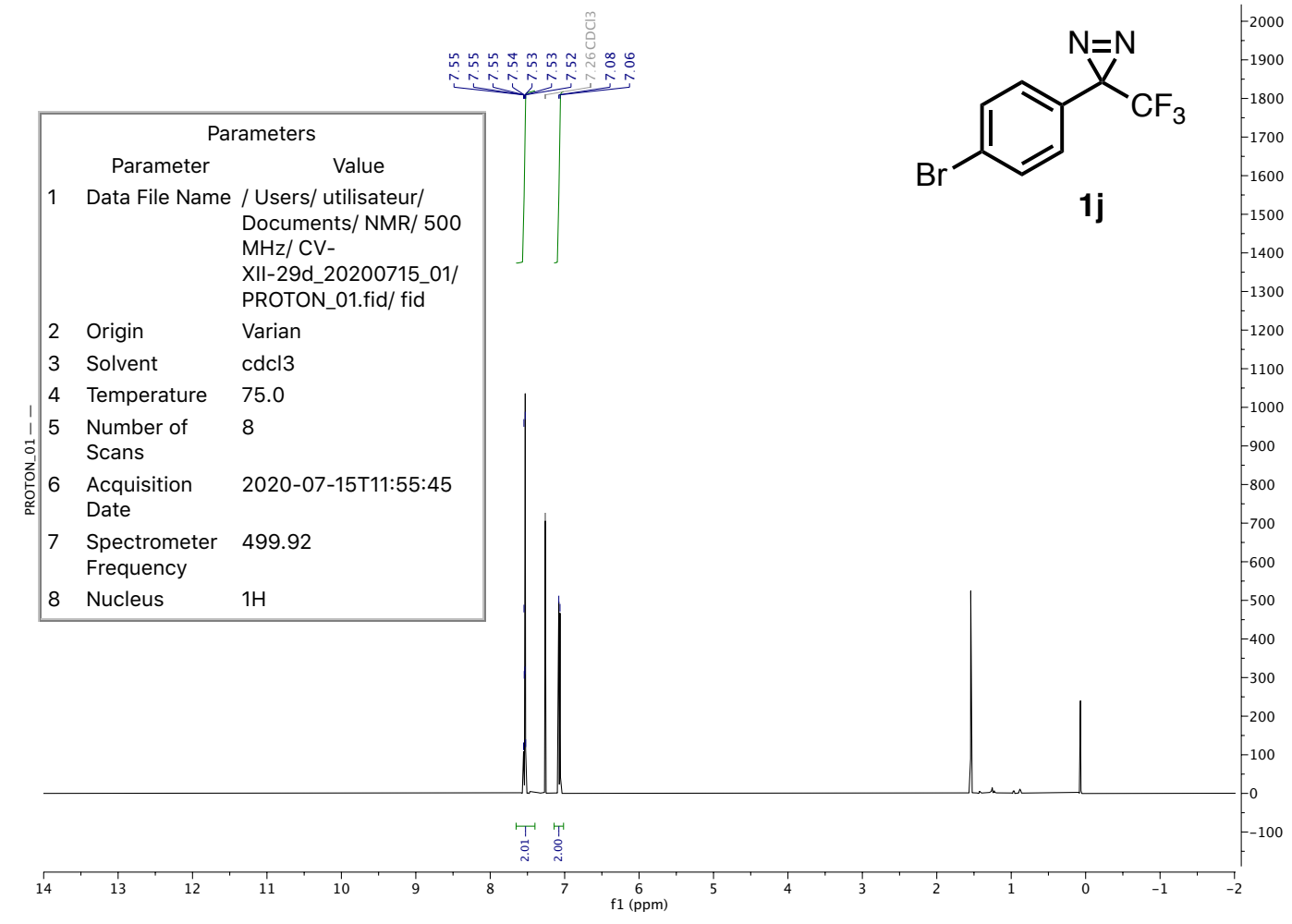




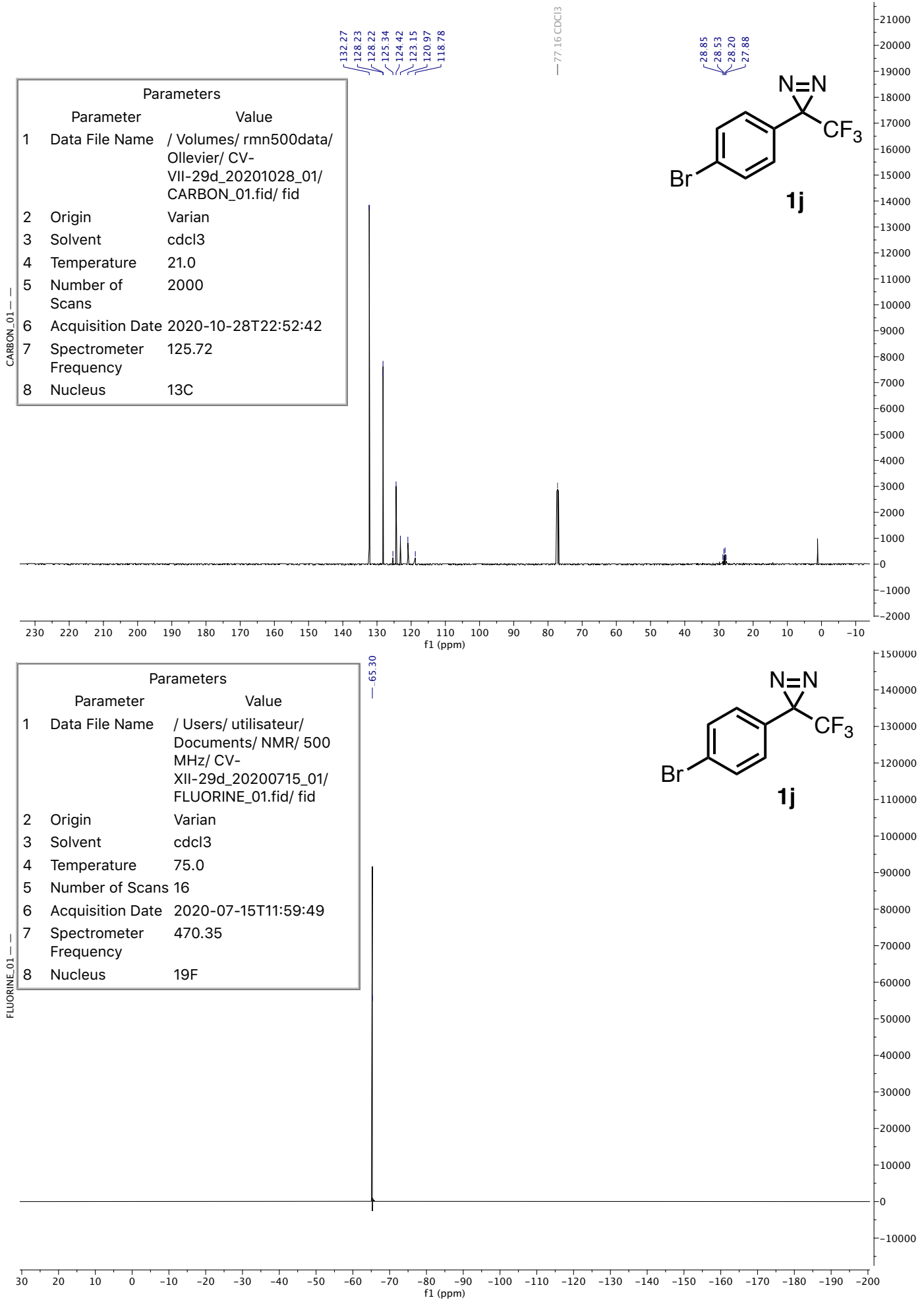




\section{3-(3-Bromophenyl)-3-(trifluoromethyl)-3H-diazirine 1k}

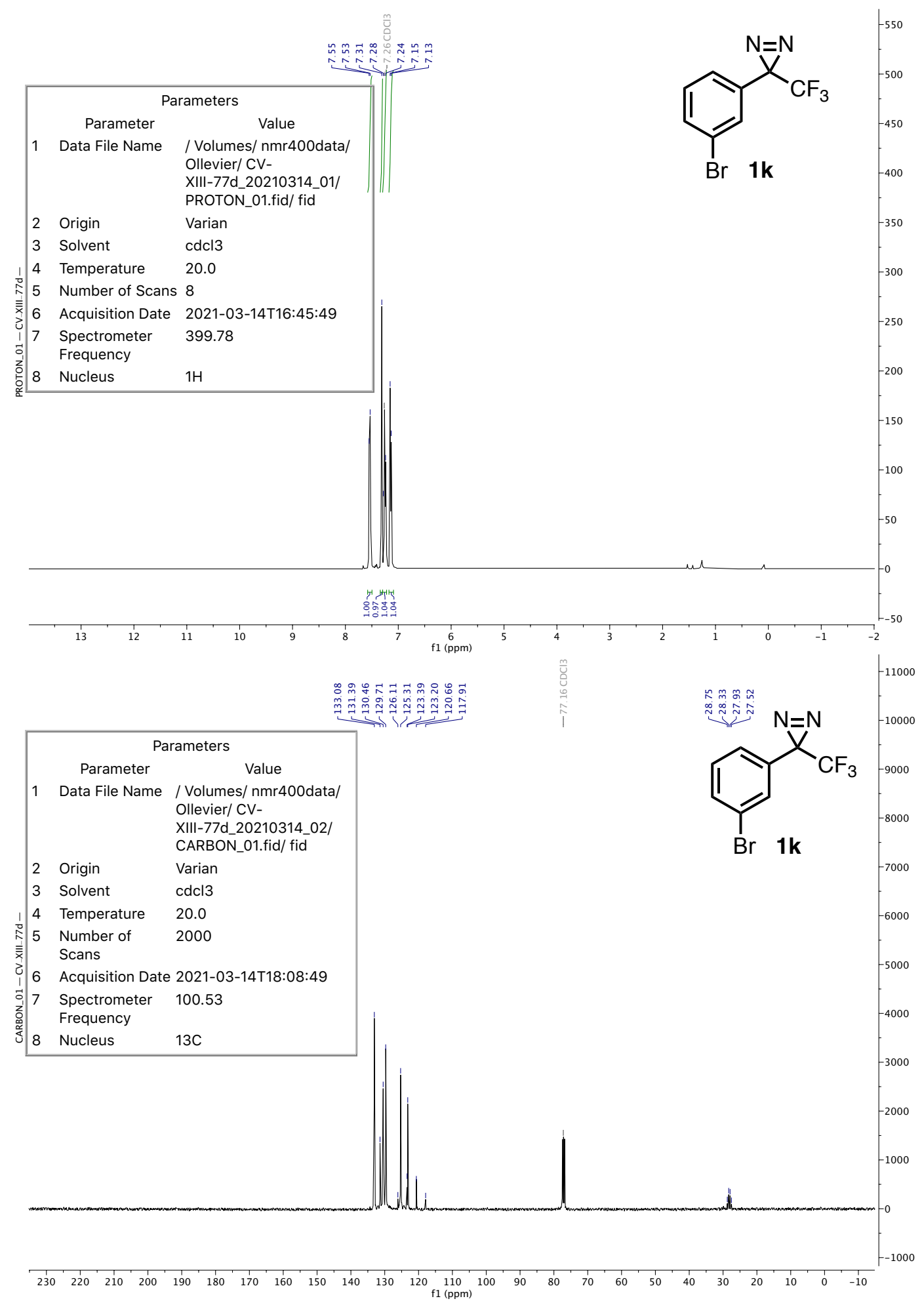




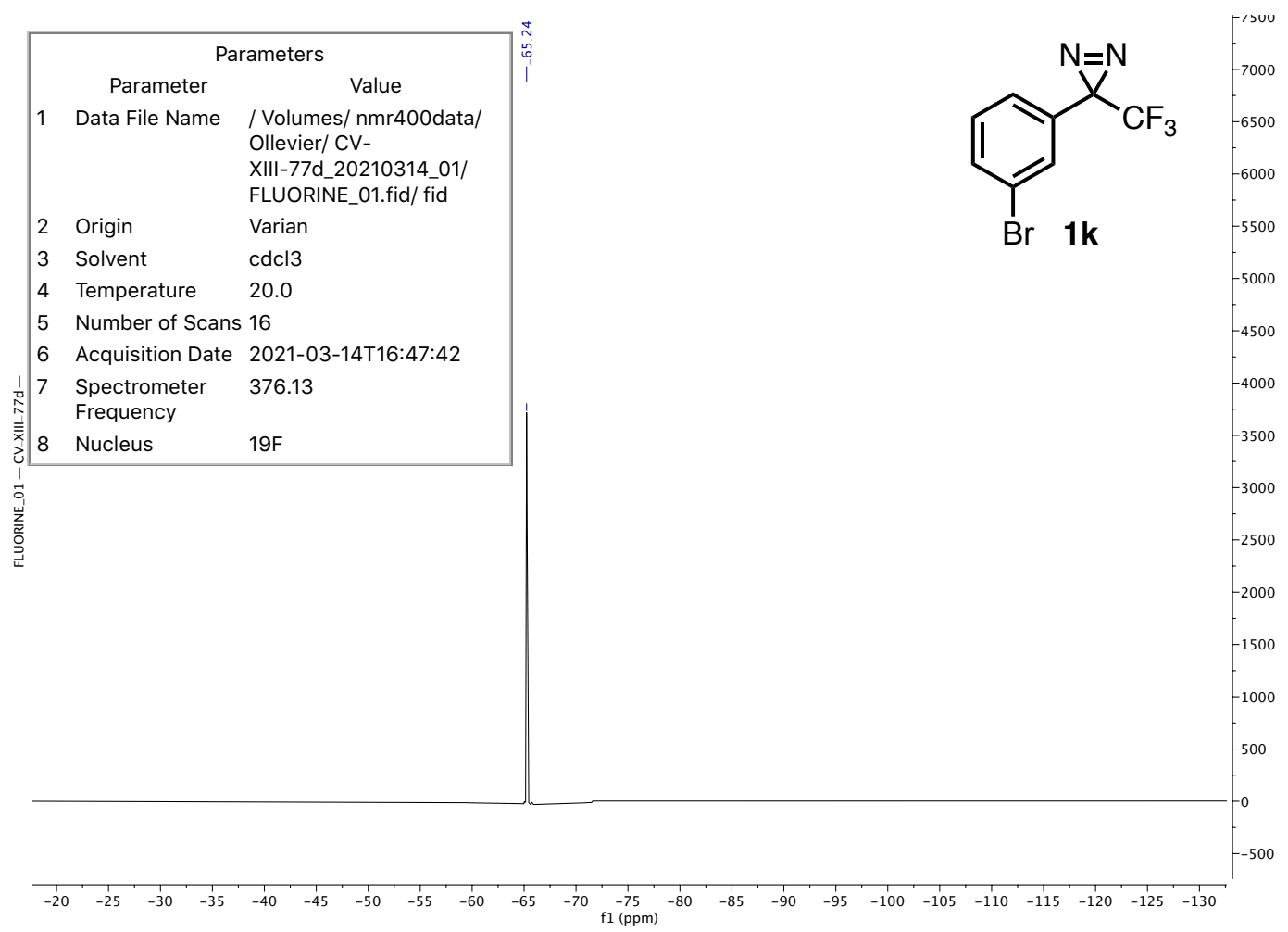

\section{3-(Trifluoromethyl)-3-(4-(trifluoromethyl)phenyl)-3H-diazirine 1l}

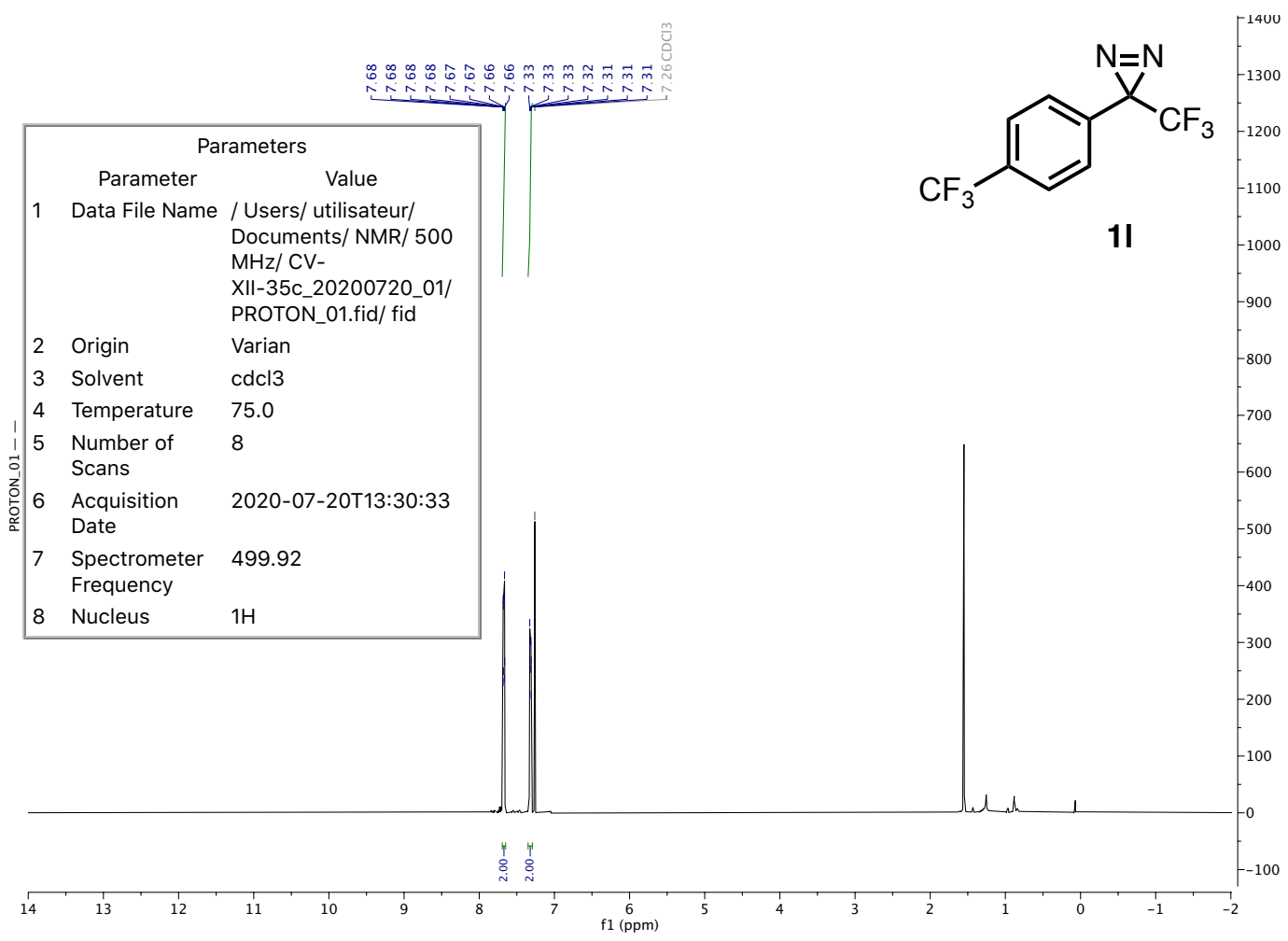




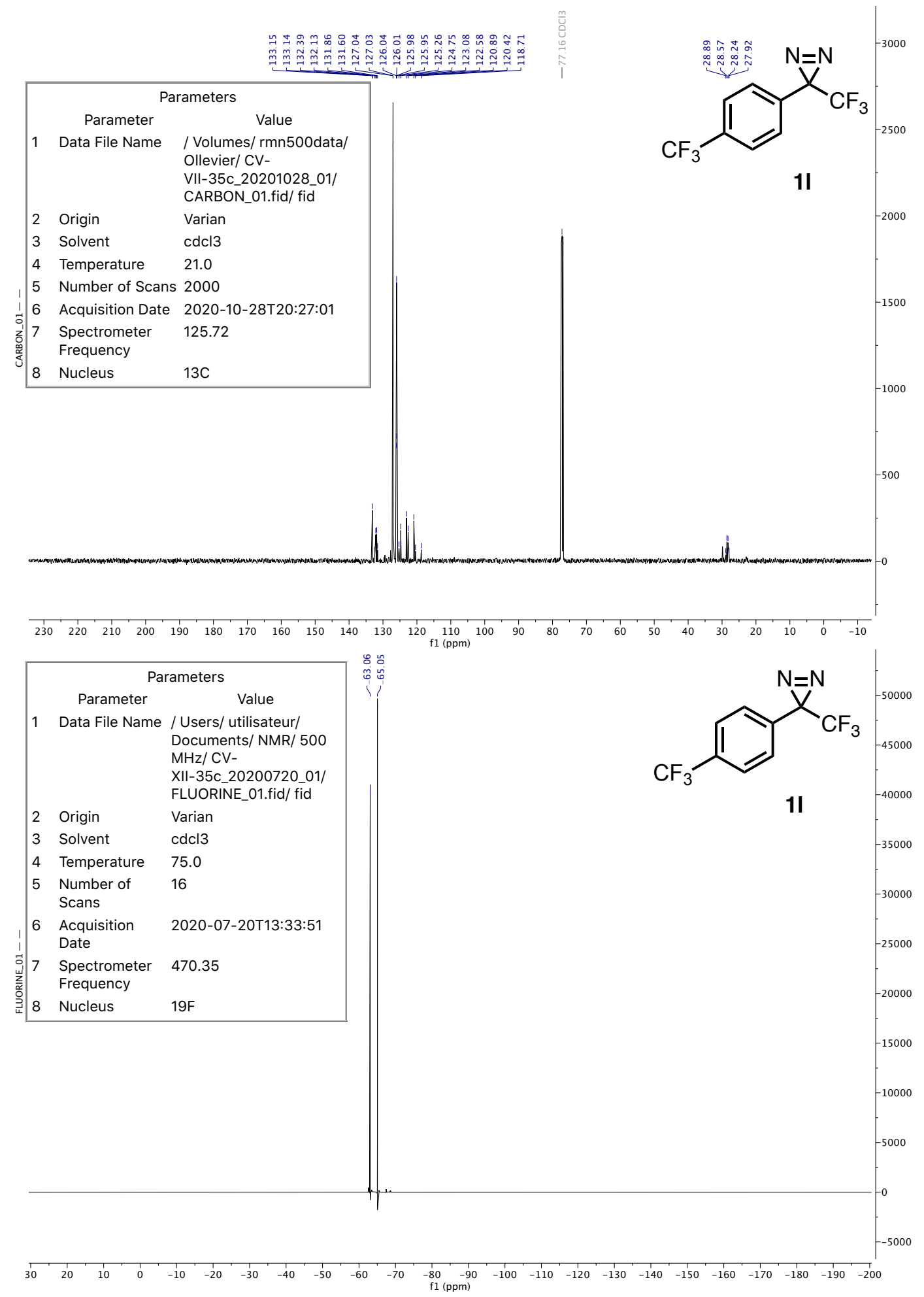




\section{3-(2-Methoxyphenyl)-3-(trifluoromethyl)-3H-diazirine $1 \mathrm{~m}$}

\begin{tabular}{|c|c|c|}
\hline \multicolumn{3}{|c|}{ Parameters } \\
\hline & Parameter & Value \\
\hline 1 & Data File Name & $\begin{array}{l}\text { / Volumes/nmr400data/ } \\
\text { Ollevier/ CV- } \\
\text { XIII-81c_20210316_01/ } \\
\text { PROTON_01.fid/fid }\end{array}$ \\
\hline 2 & Origin & Varian \\
\hline 3 & Solvent & cdcl3 \\
\hline 4 & Temperature & 20.0 \\
\hline 5 & Number of Scans & 8 \\
\hline 6 & Acquisition Date & 2021-03-16Т01:09:54 \\
\hline 7 & $\begin{array}{l}\text { Spectrometer } \\
\text { Frequency }\end{array}$ & 399.78 \\
\hline 8 & Nucleus & $1 \mathrm{H}$ \\
\hline
\end{tabular}

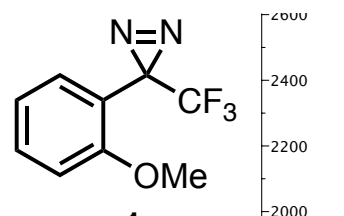

$1 \mathrm{~m}$

800

600

400

1200

000

$-800$

$-600$

$-400$

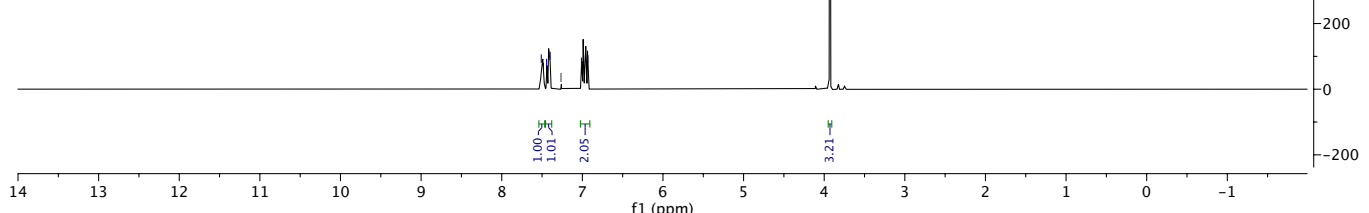

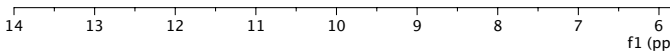

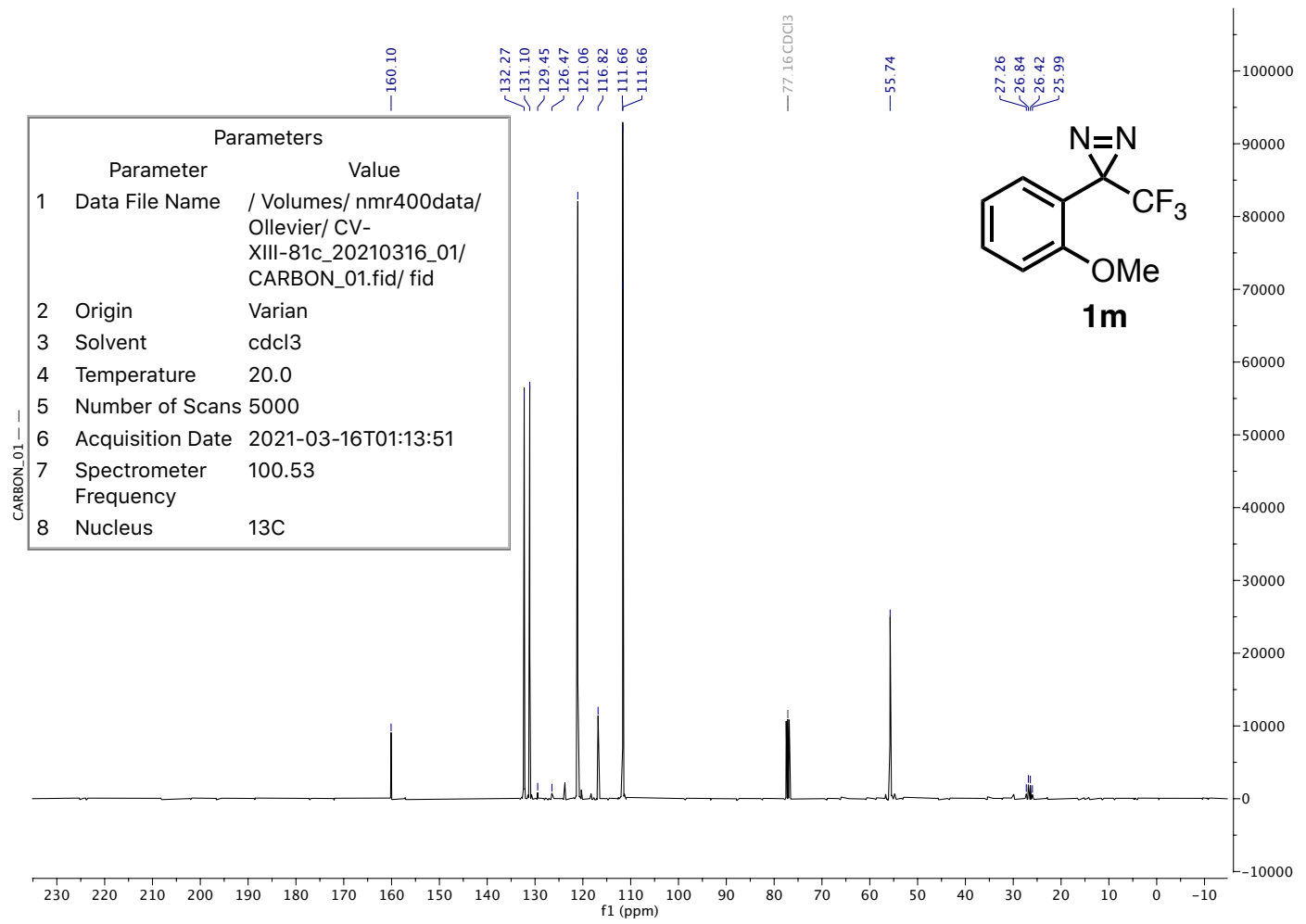




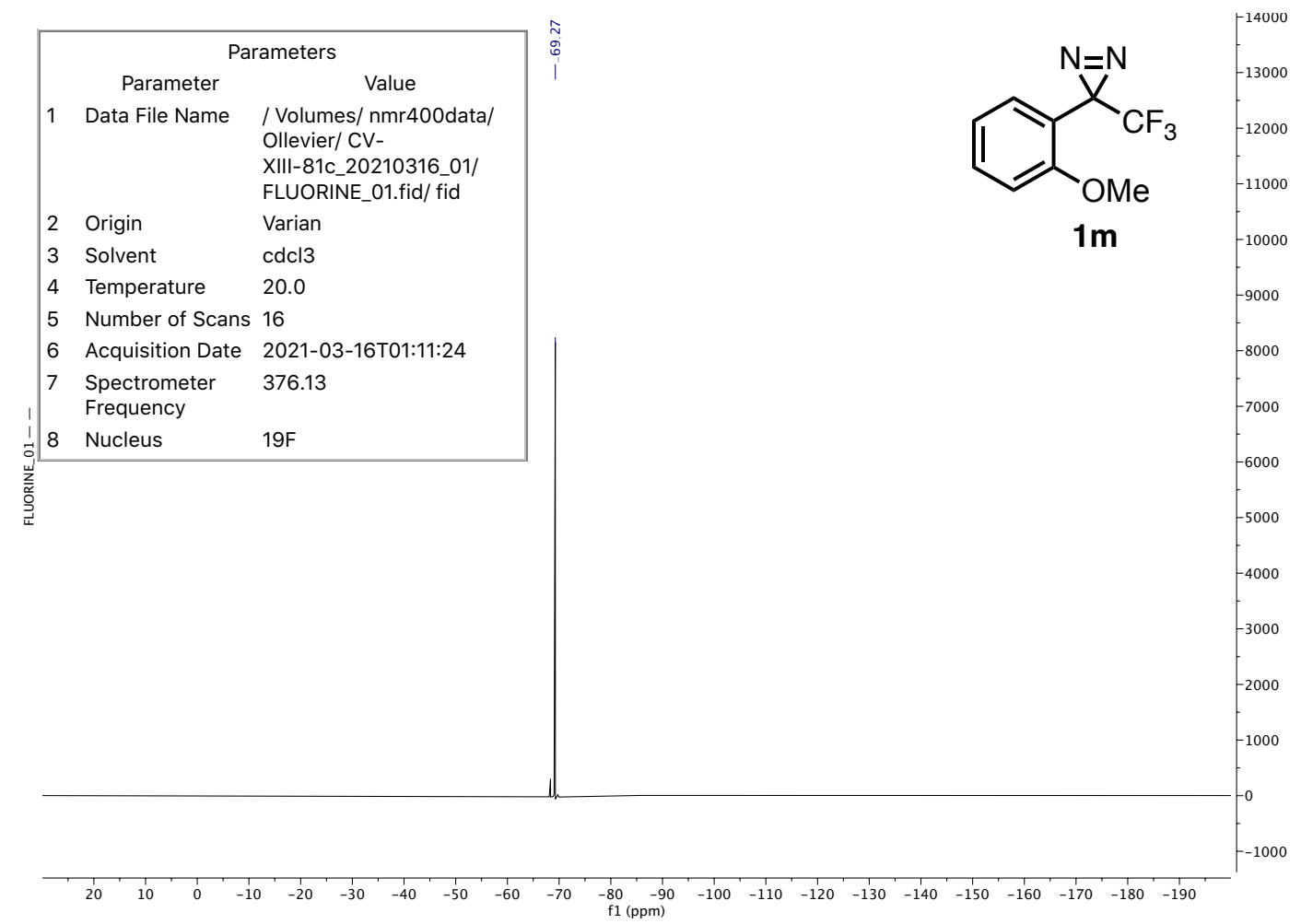

\section{3-(2-Fluorophenyl)-3-(trifluoromethyl)-3H-diazirine 1n}

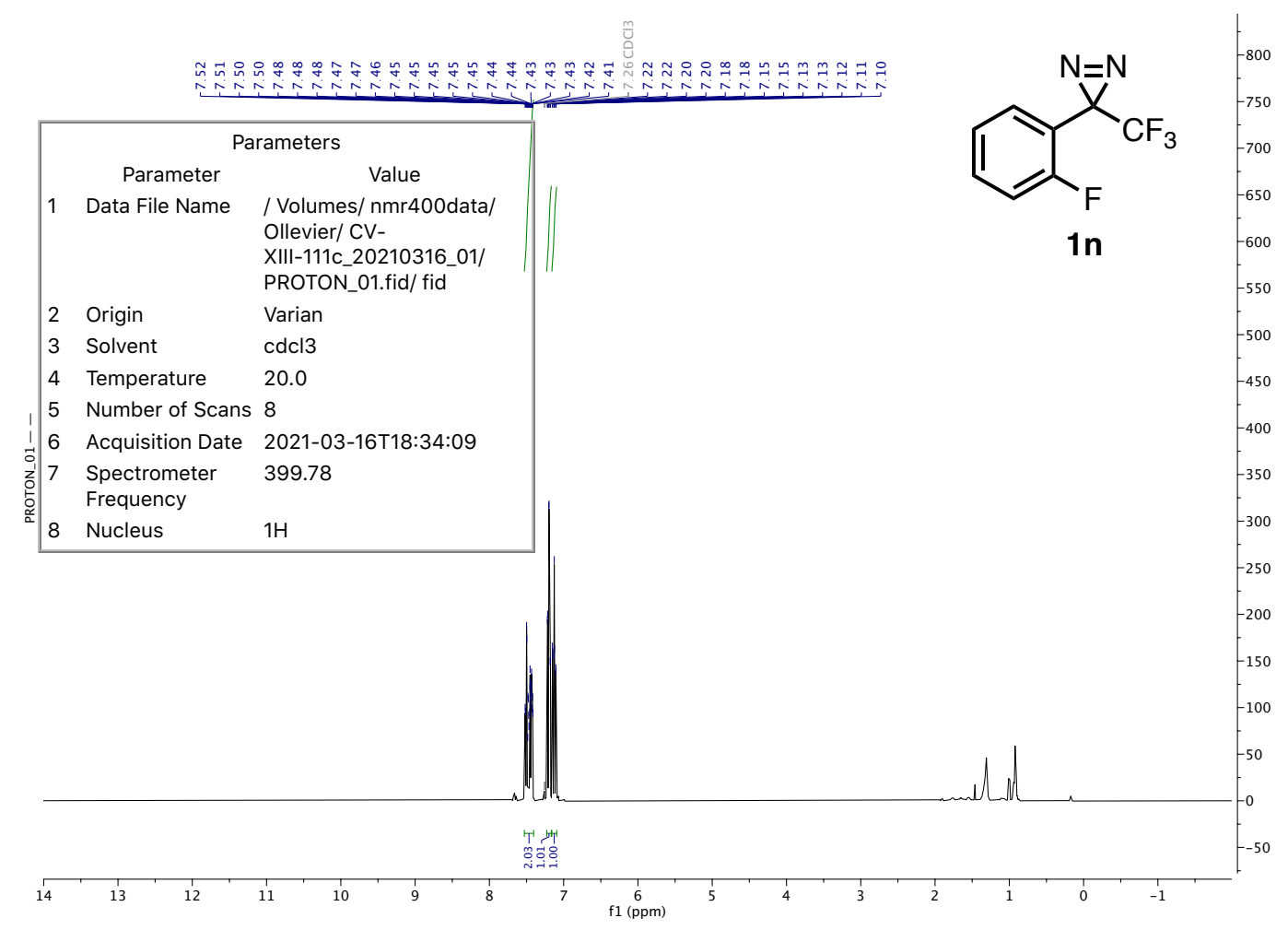




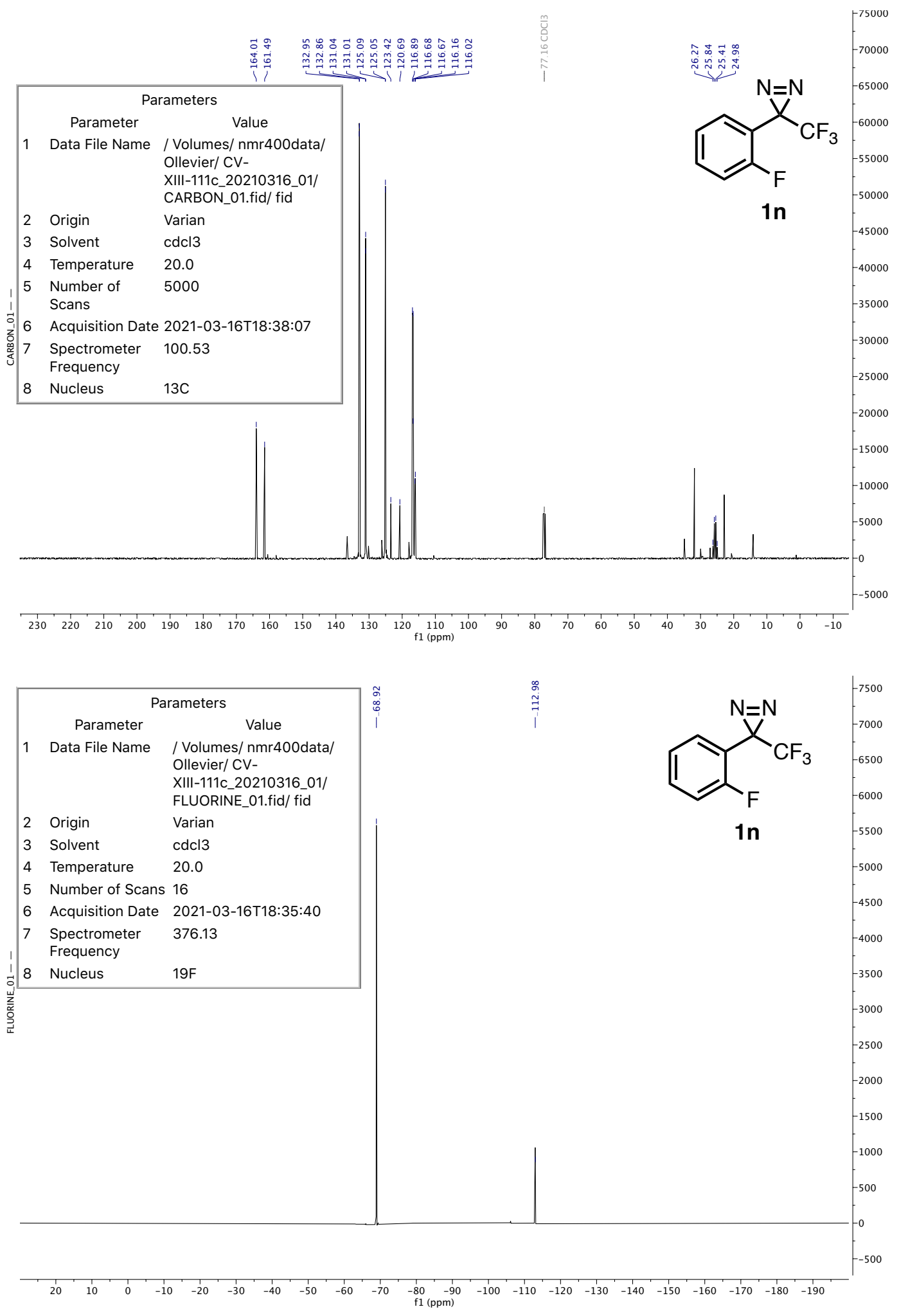




\section{4-(2,3-Diphenyl-1-(trifluoromethyl)cycloprop-2-en-1-yl)-1,1'-biphenyl 2a}

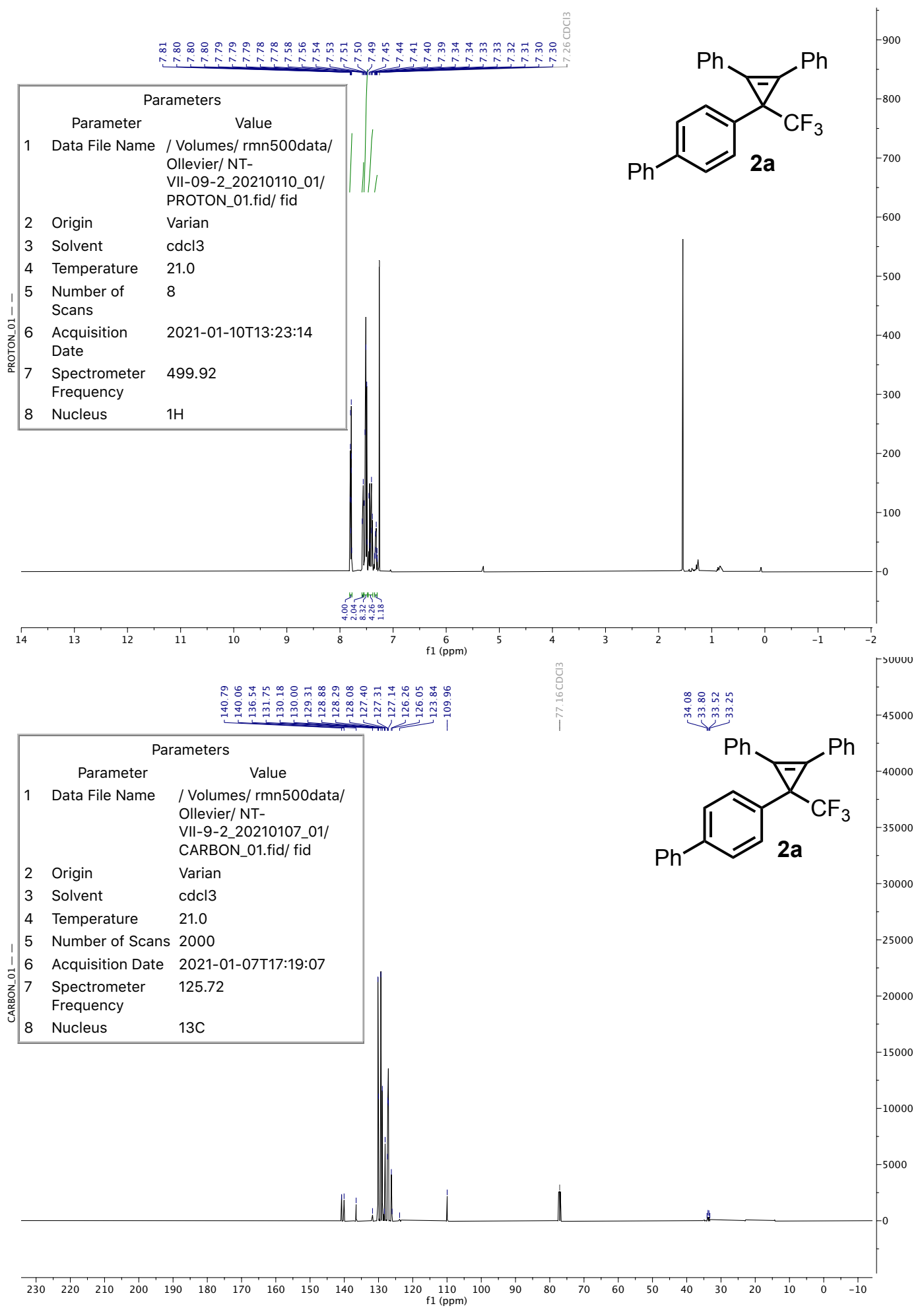




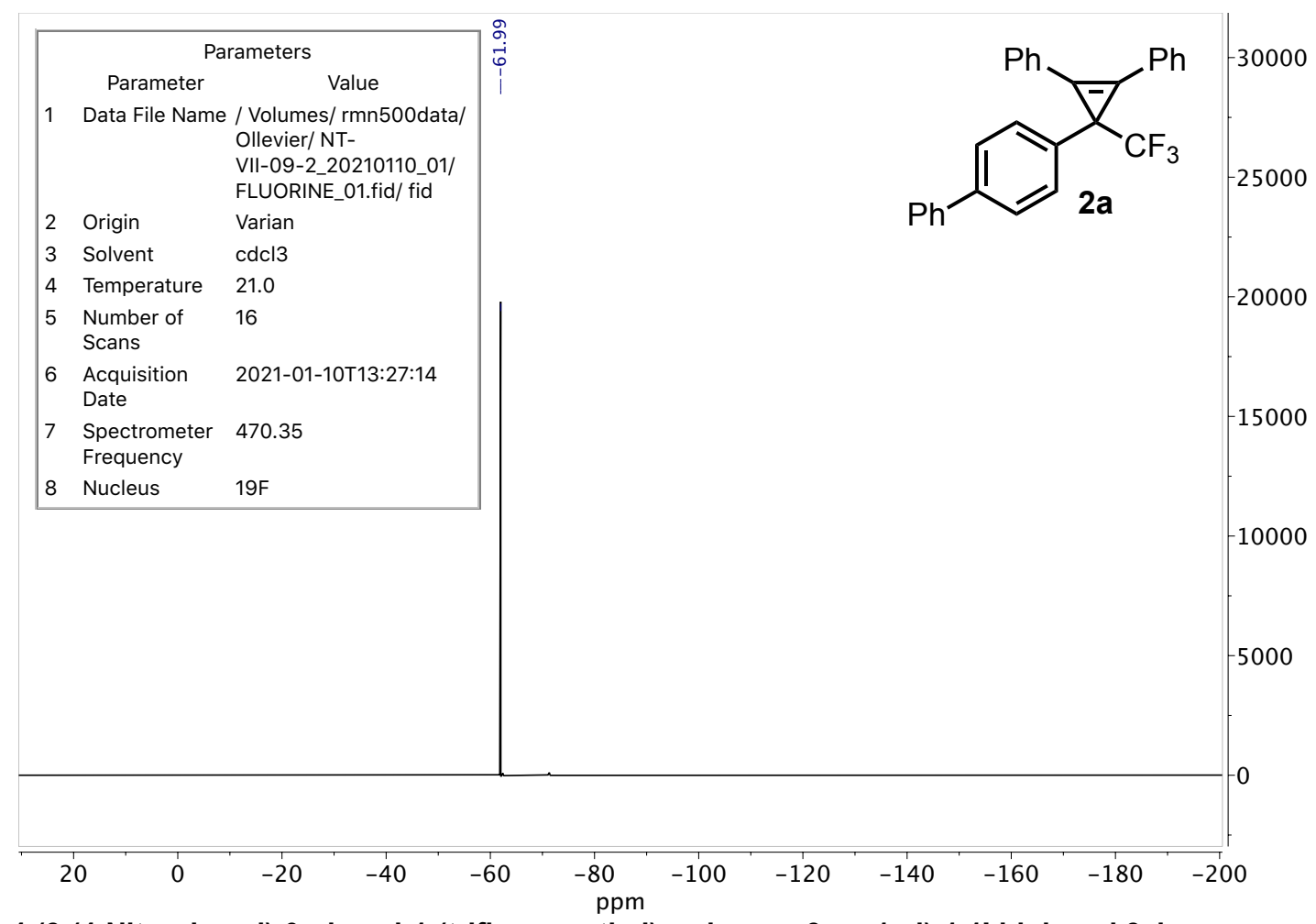

\section{4-(2-(4-Nitrophenyl)-3-phenyl-1-(trifluoromethyl)cycloprop-2-en-1-yl)-1,1'-biphenyl 2ab}

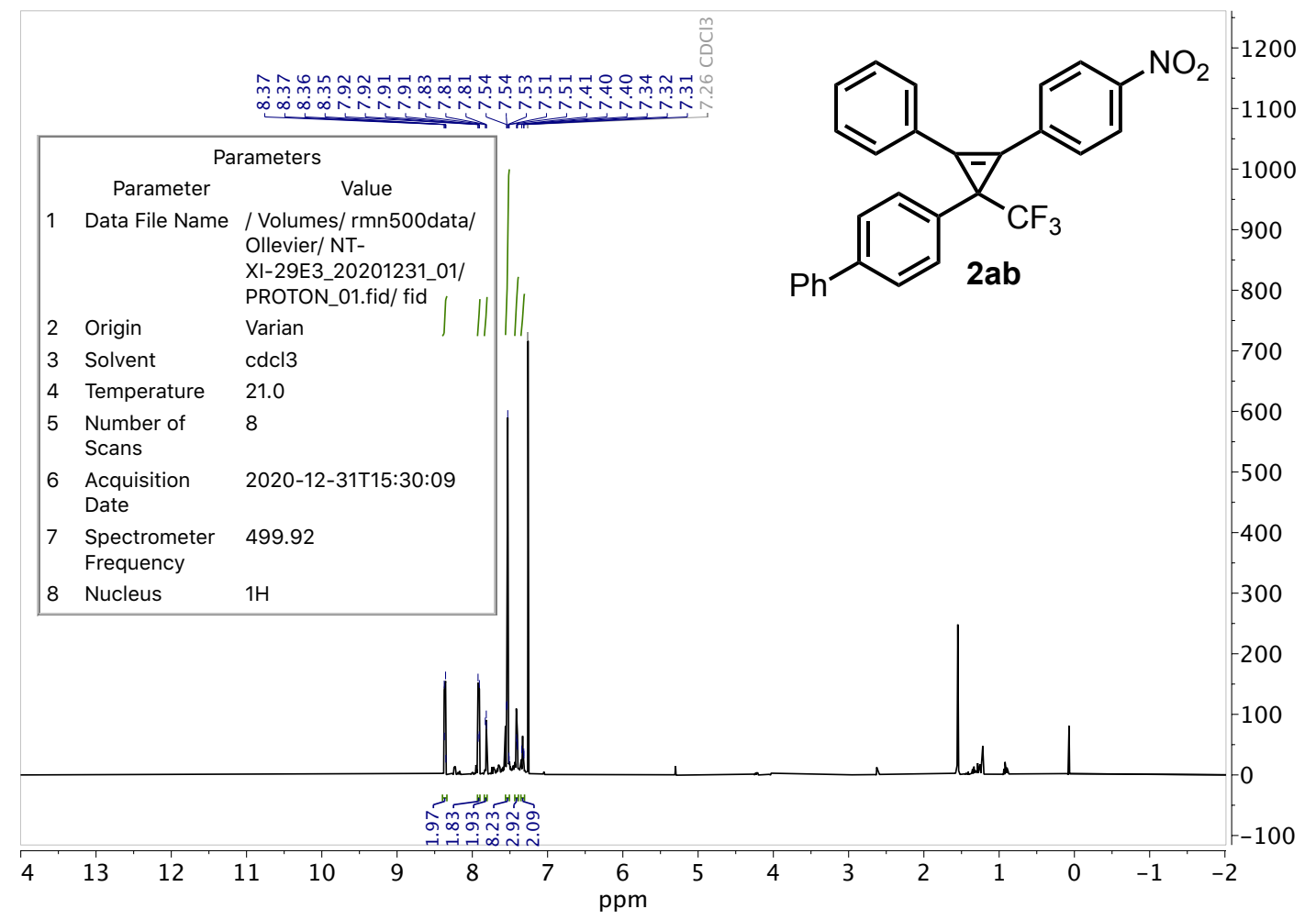




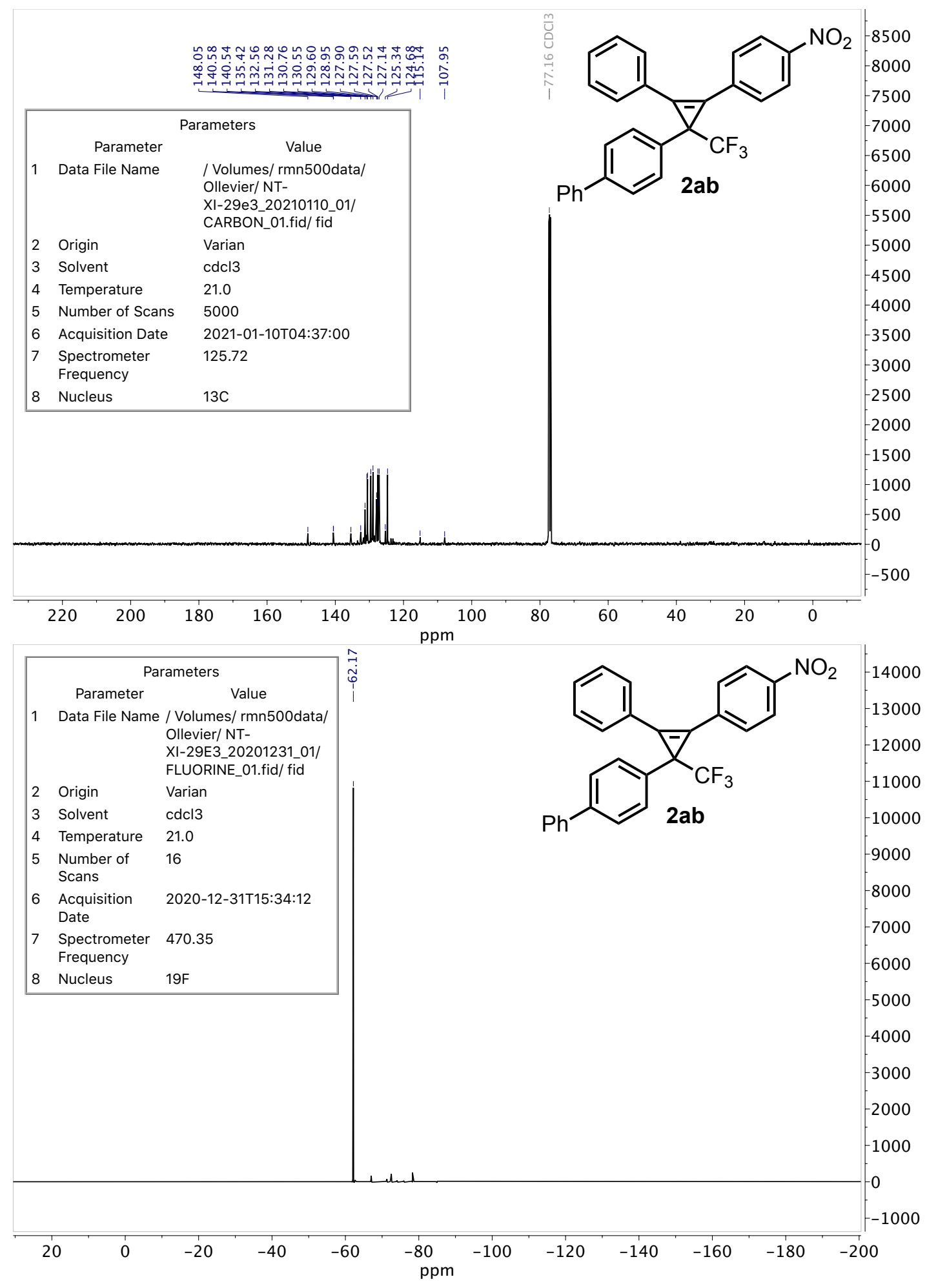


1-(4-(3-([1,1'-Biphenyl]-4-yl)-2-phenyl-3-(trifluoromethyl)cycloprop-1-en-1-yl)phenyl)ethan-1-one 2ac

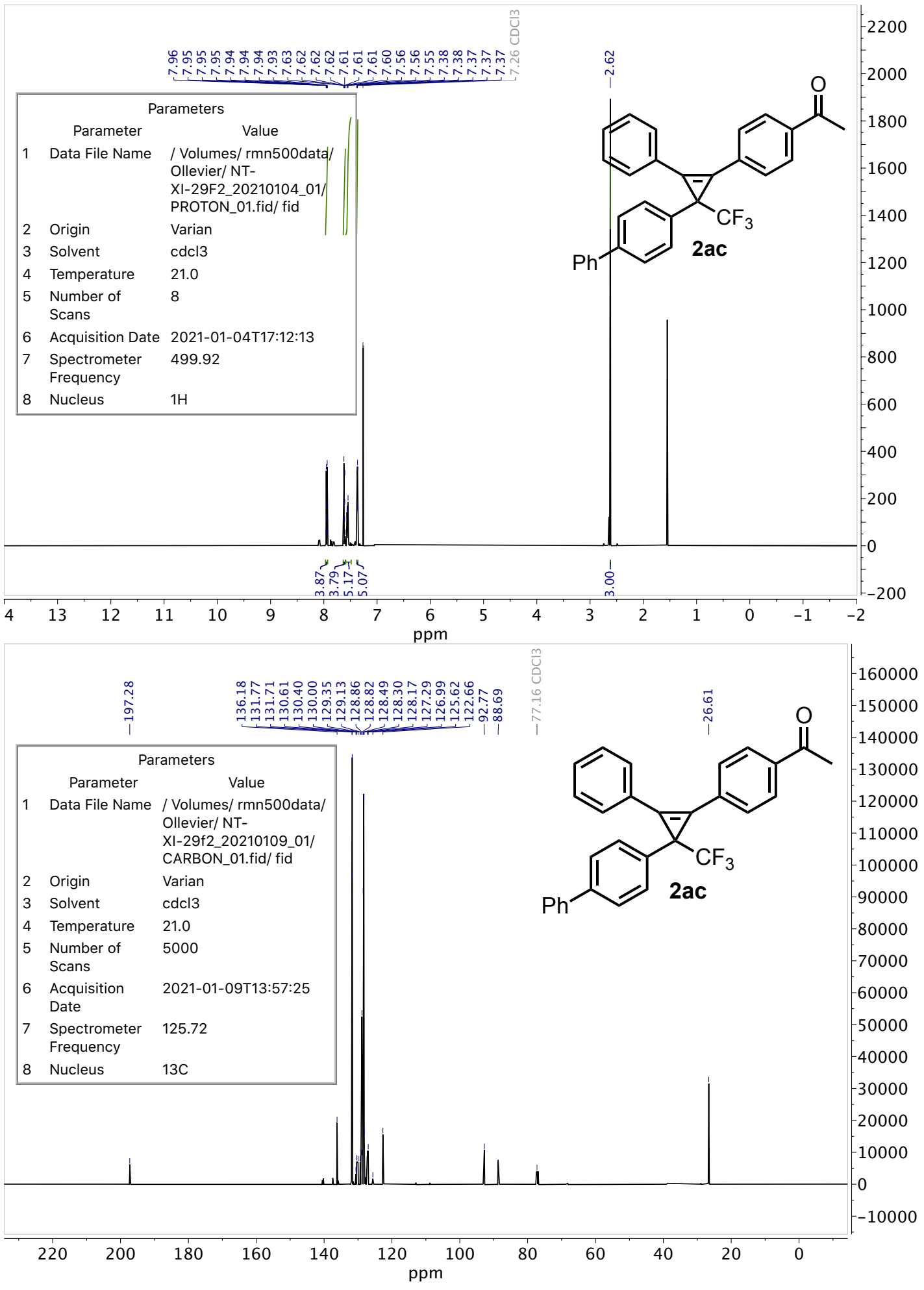




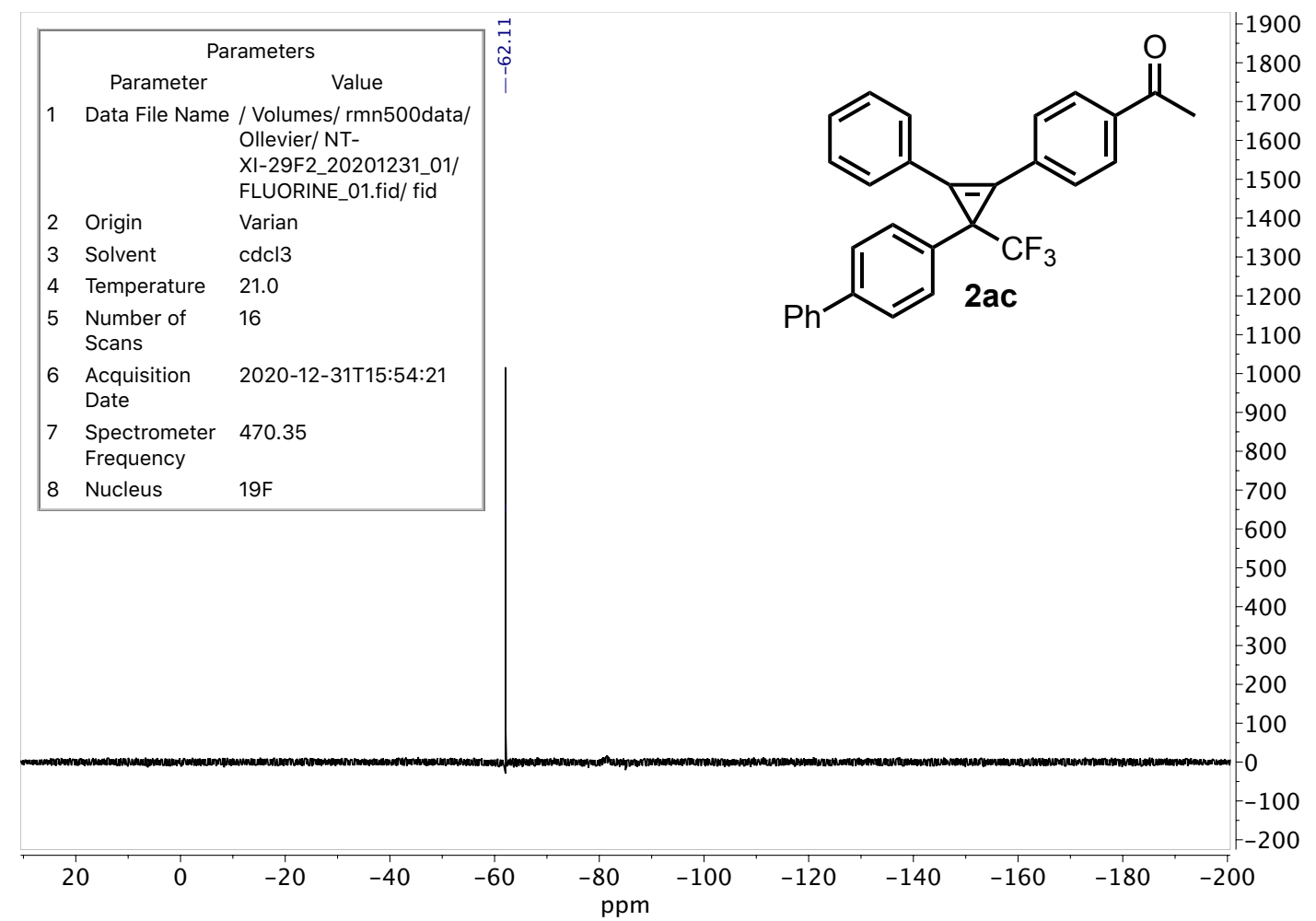

4-(2-Phenyl-1-(trifluoromethyl)-3-(4-(trifluoromethyl)phenyl)cycloprop-2-en-1-yl)-1,1'-biphenyl 2ad

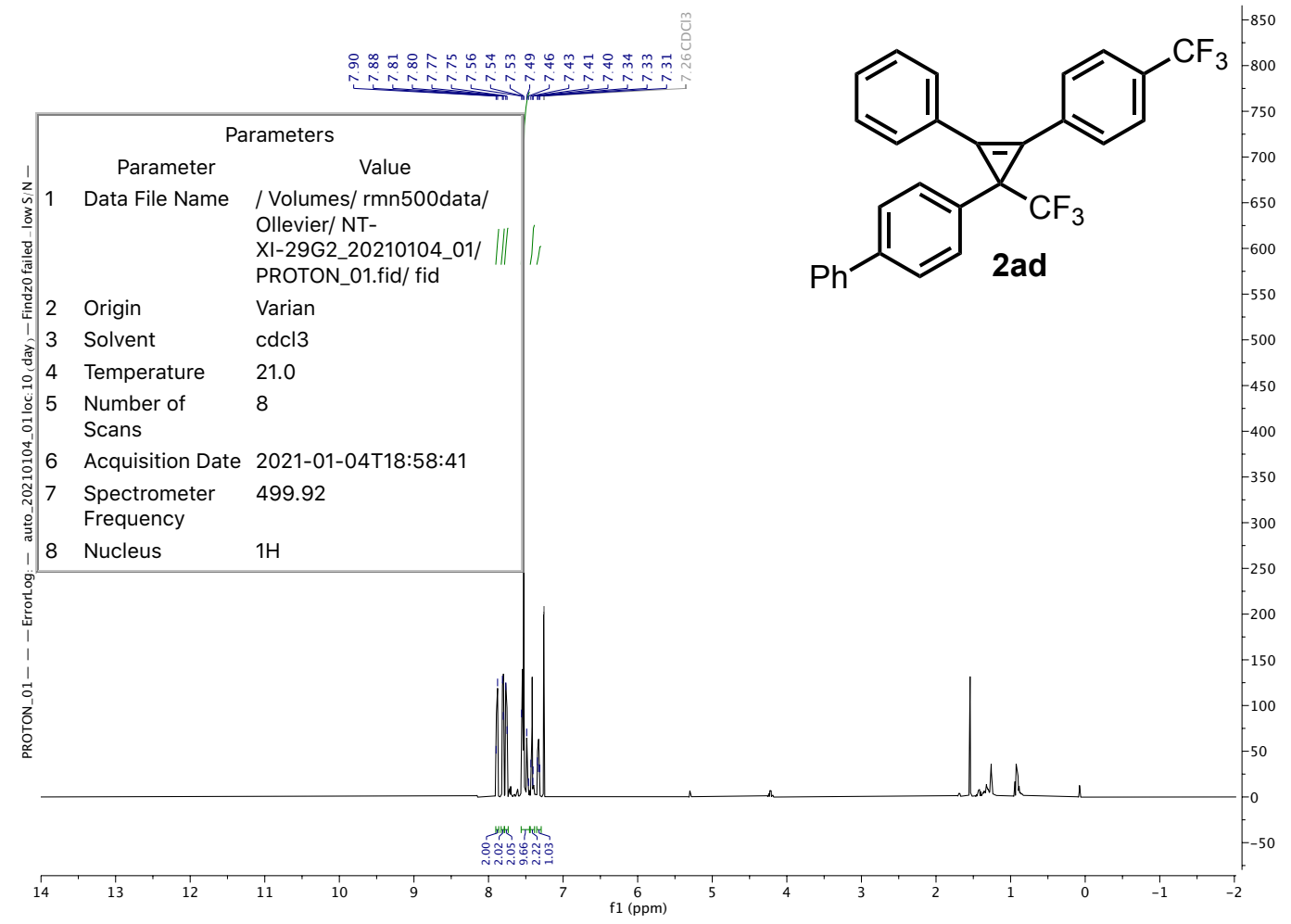




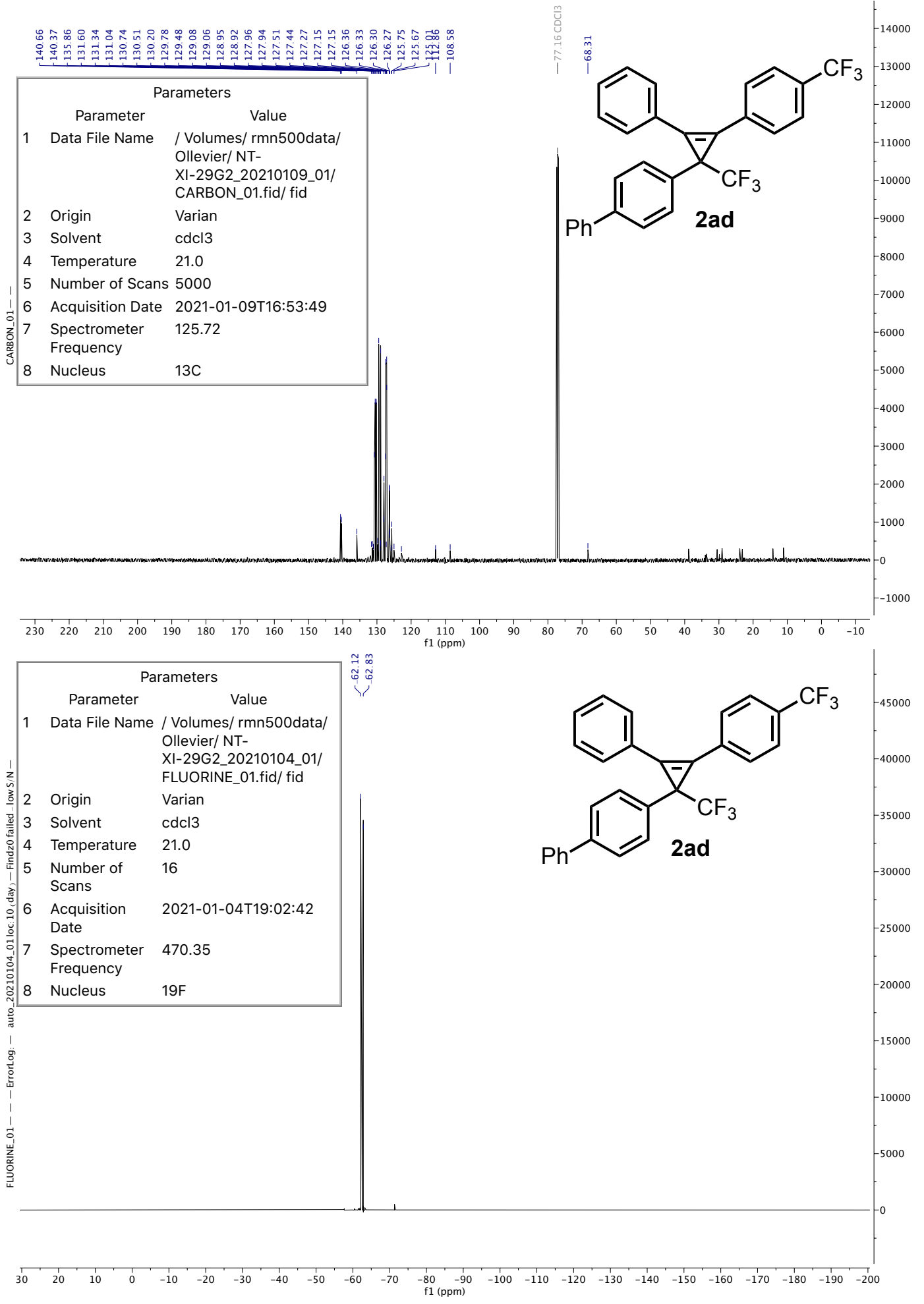




\section{4-(2-(4-Fluorophenyl)-3-phenyl-1-(trifluoromethyl)cycloprop-2-en-1-yl)-1,1'-biphenyl 2ae}

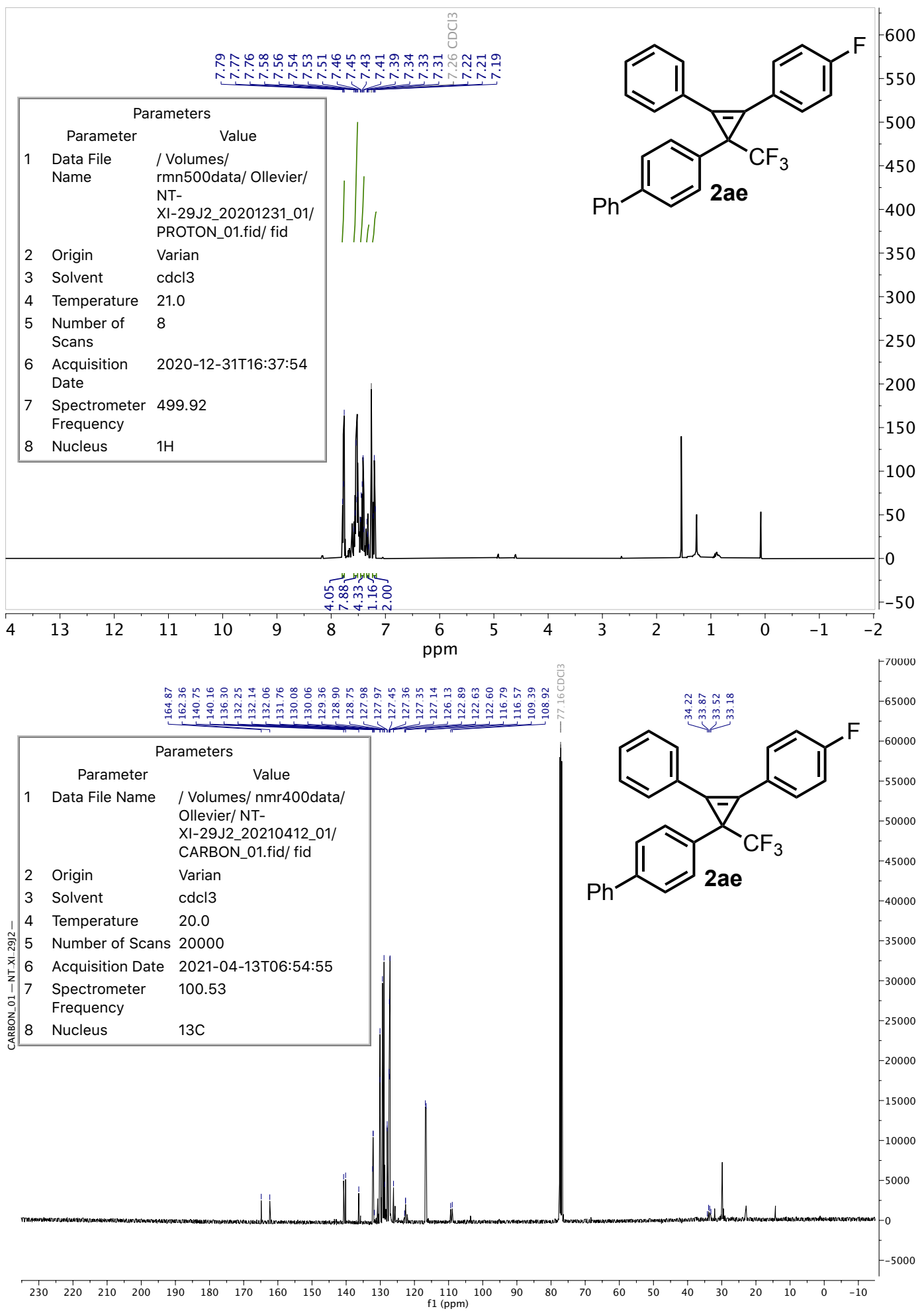




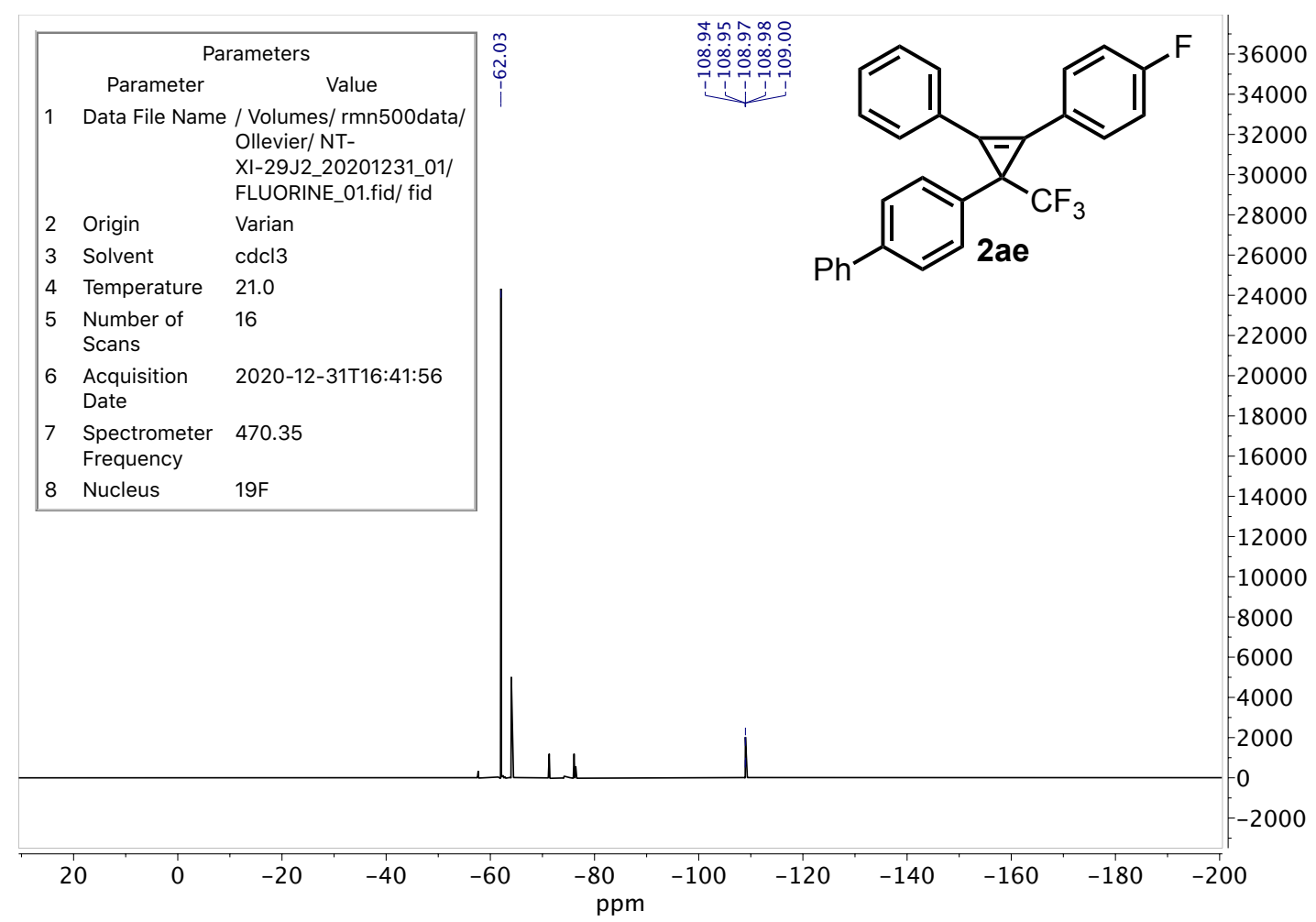

\section{4-(2,3-Dipropyl-1-(trifluoromethyl)cycloprop-2-en-1-yl)-1,1'-biphenyl 2af}

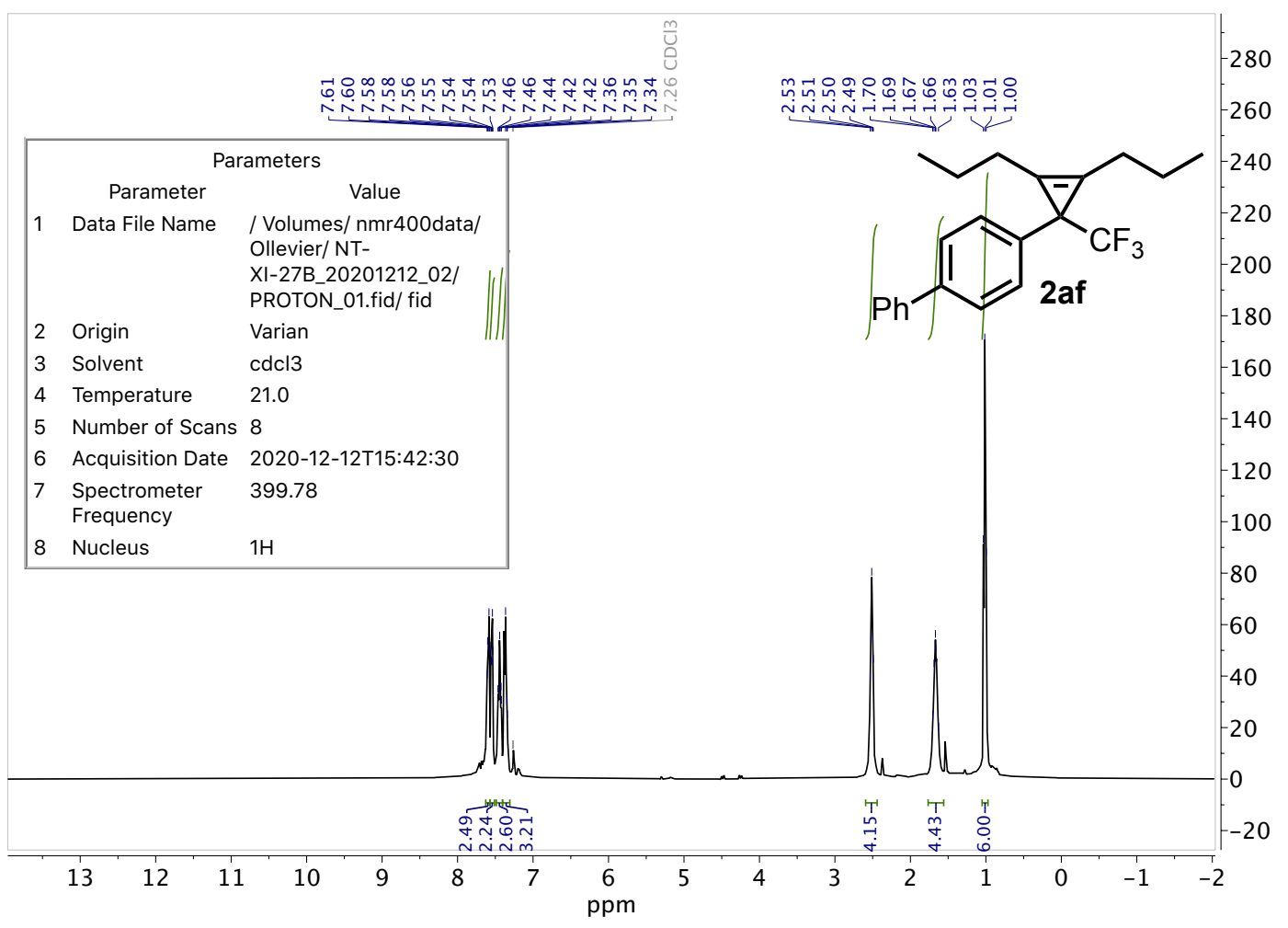




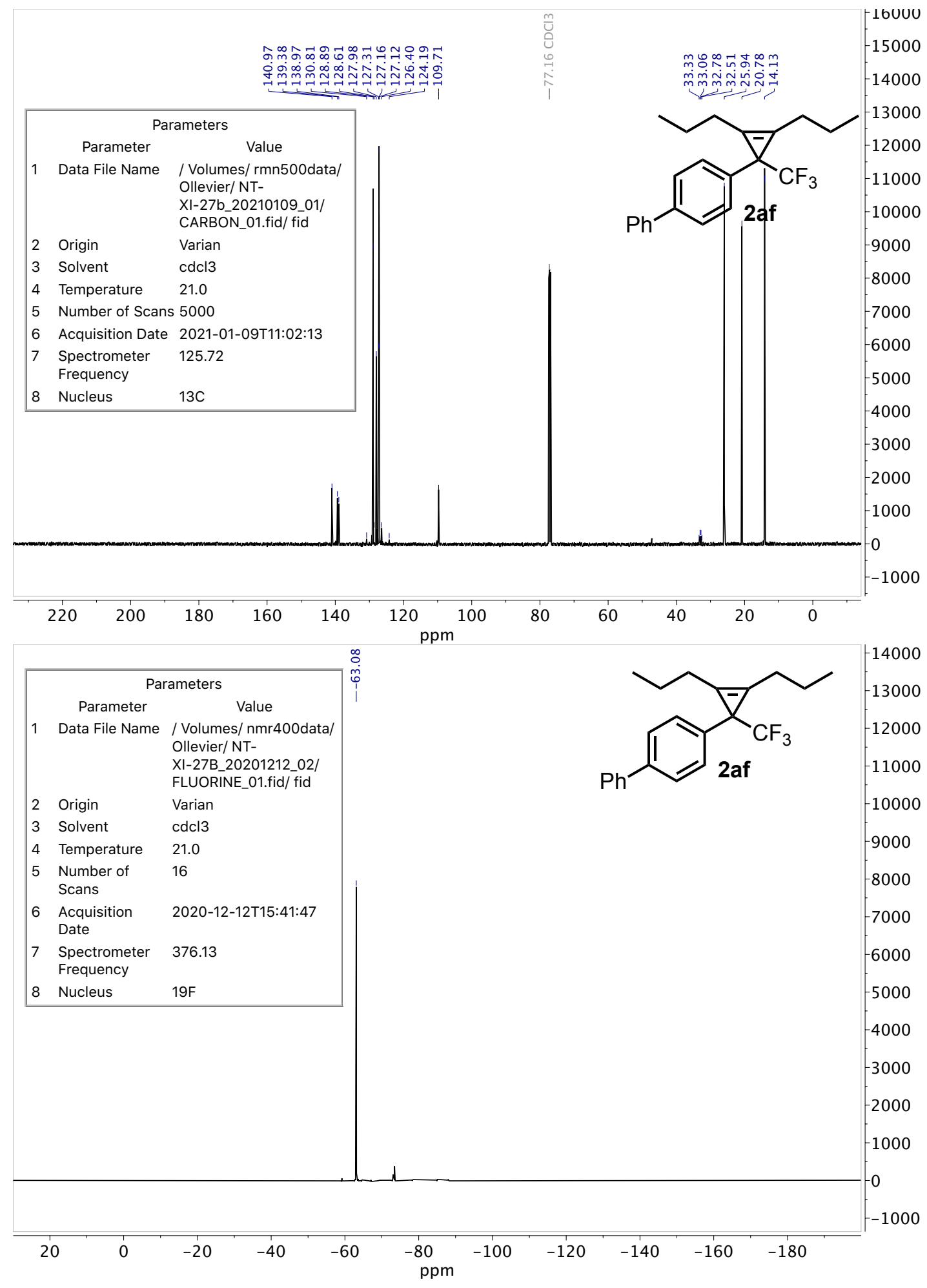




\section{4-(2-Phenyl-1-(trifluoromethyl)cycloprop-2-en-1-yl)-1,1'-biphenyl 2ag}

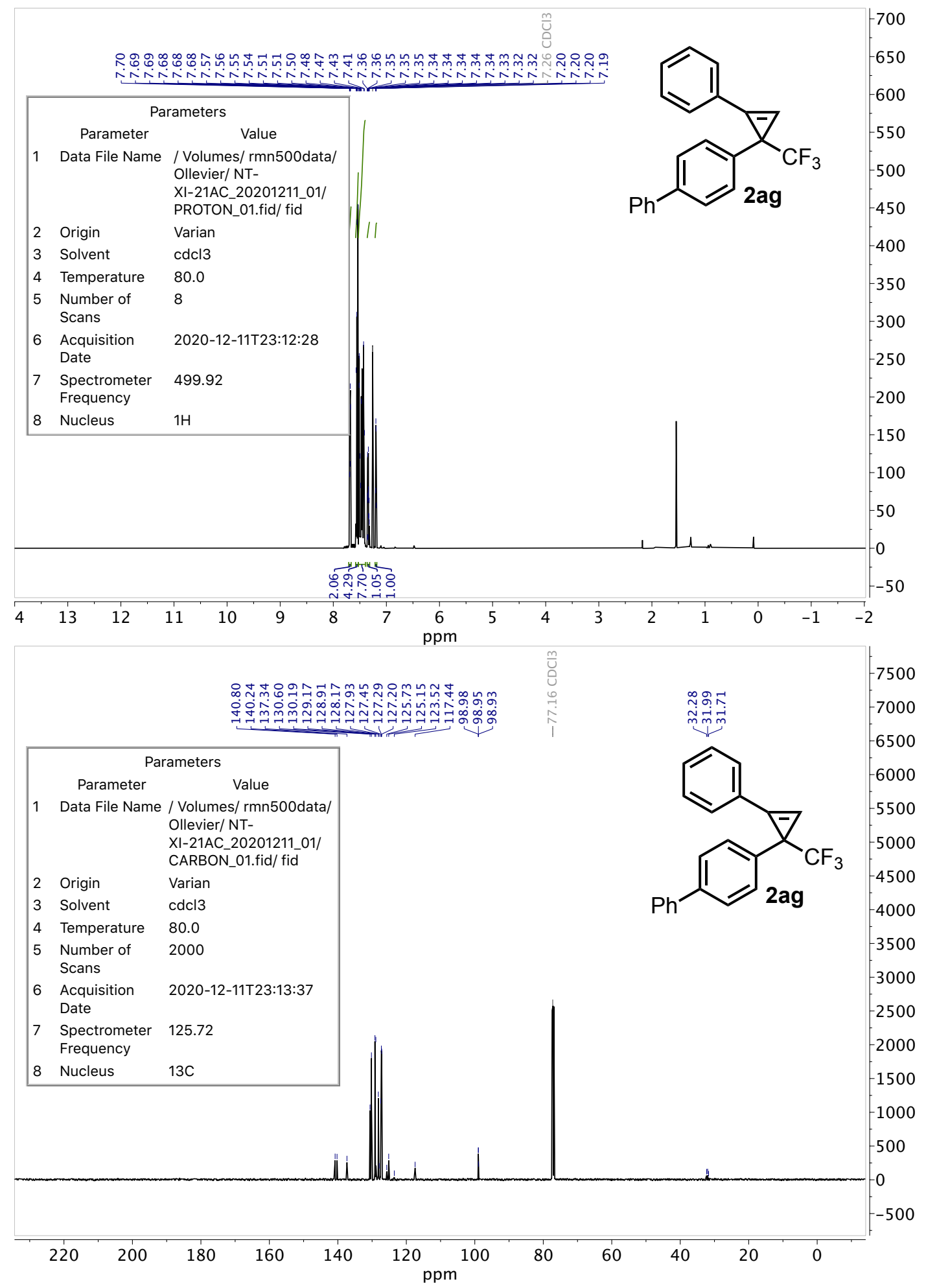




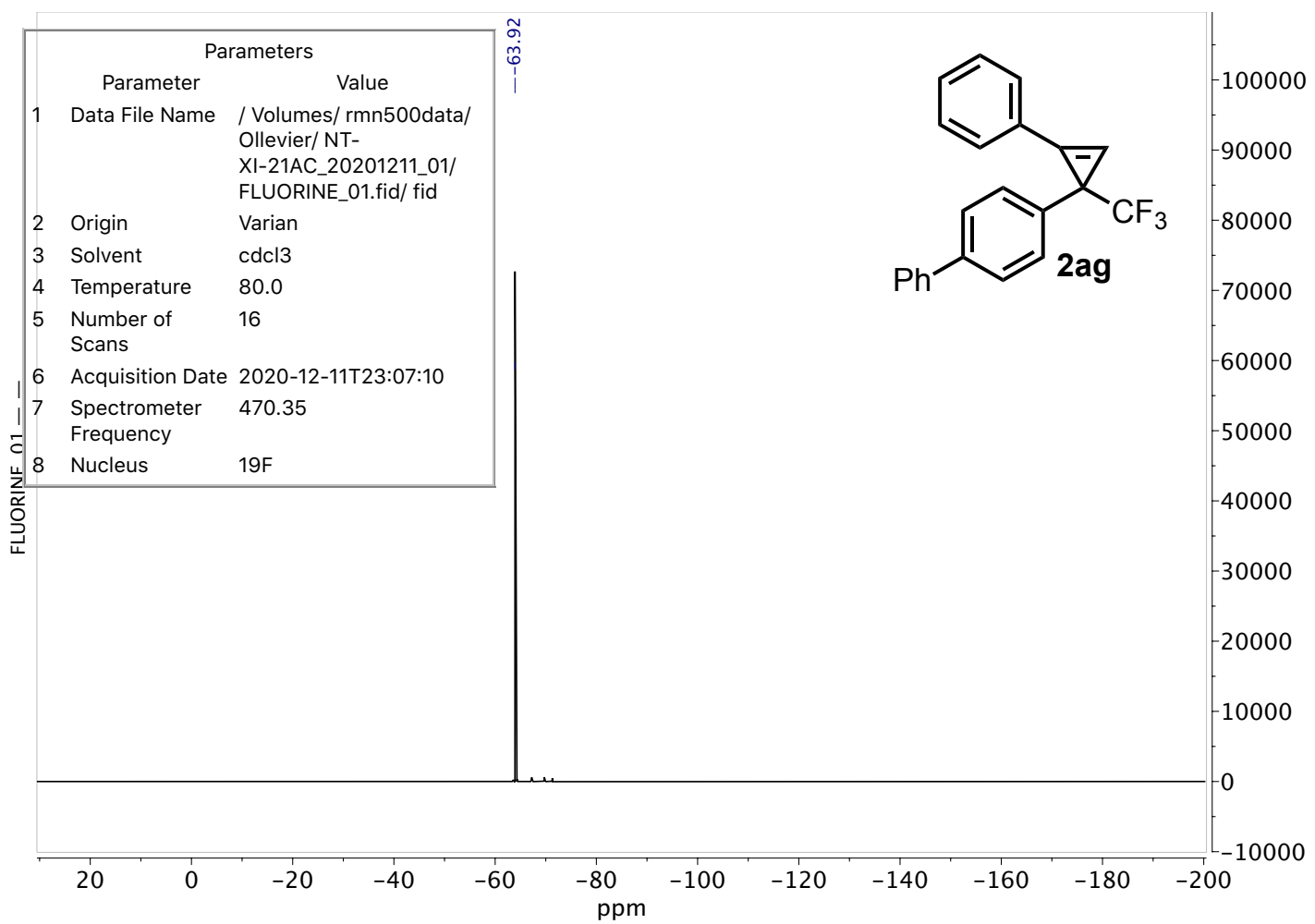

\section{4-(2-(p-Tolyl)-1-(trifluoromethyl)cycloprop-2-en-1-yl)-1,1'-biphenyl 2ah}

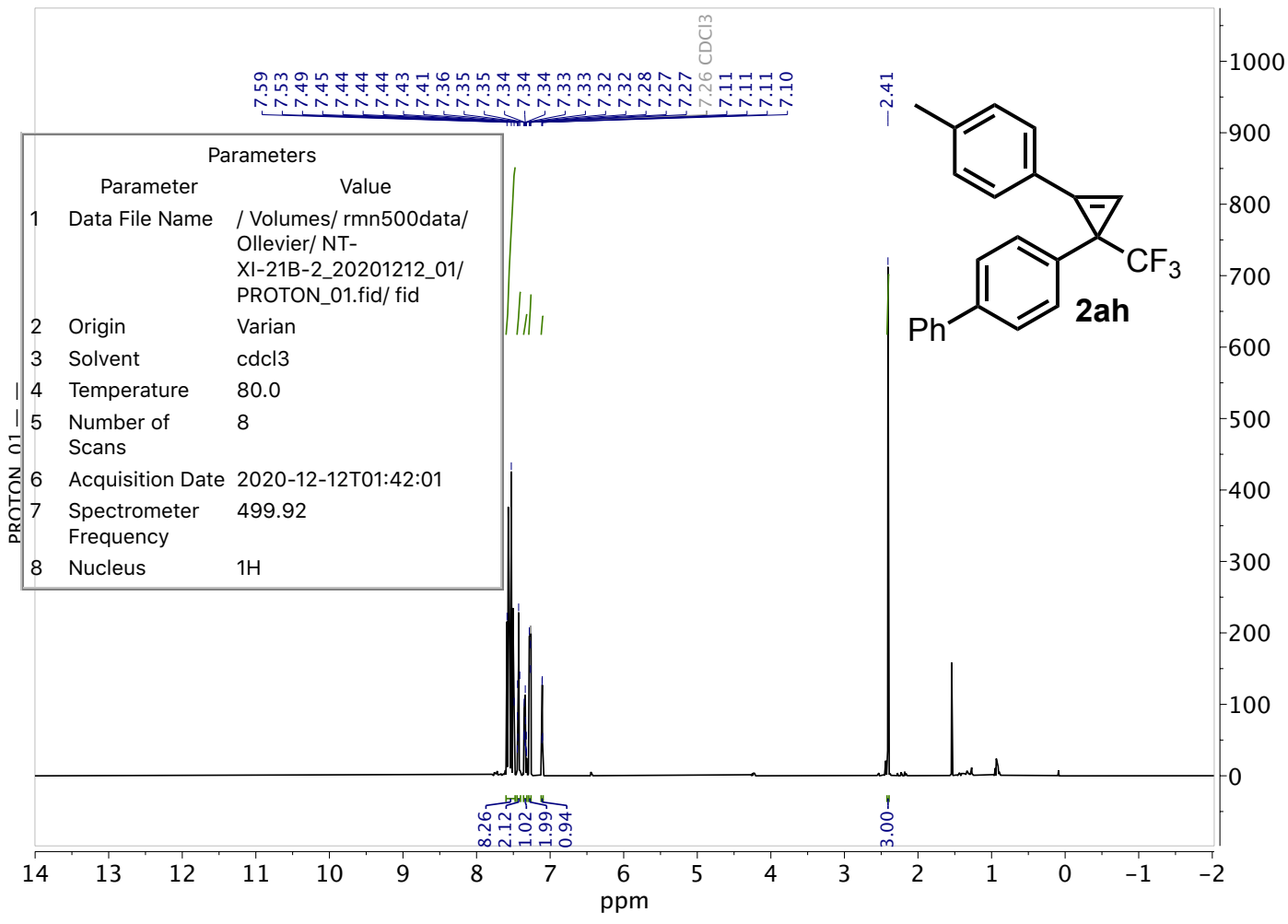




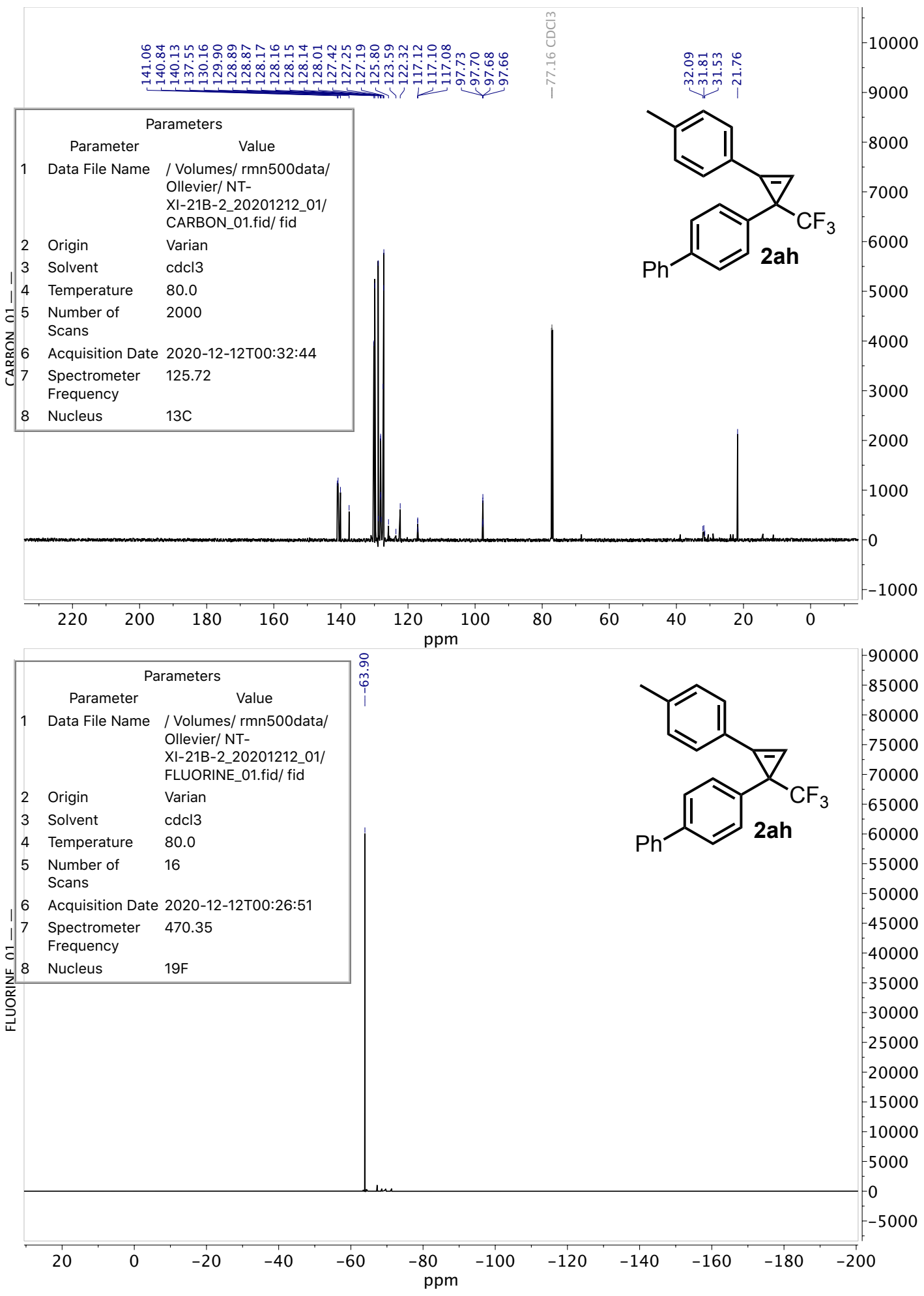




\section{4-(2-(4-Ethy|phenyl)-1-(trifluoromethyl)cycloprop-2-en-1-yl)-1,1'-biphenyl 2ai}

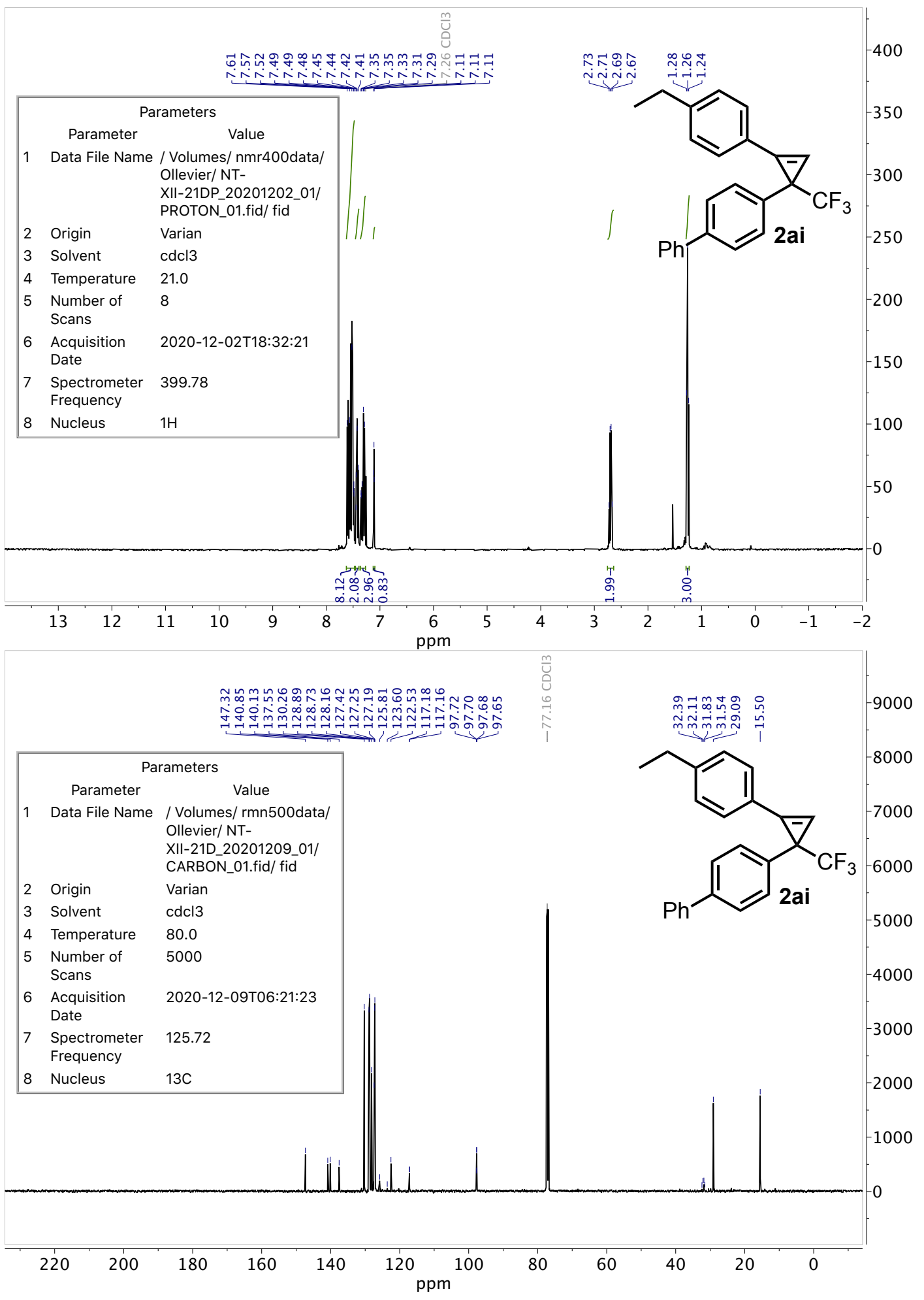




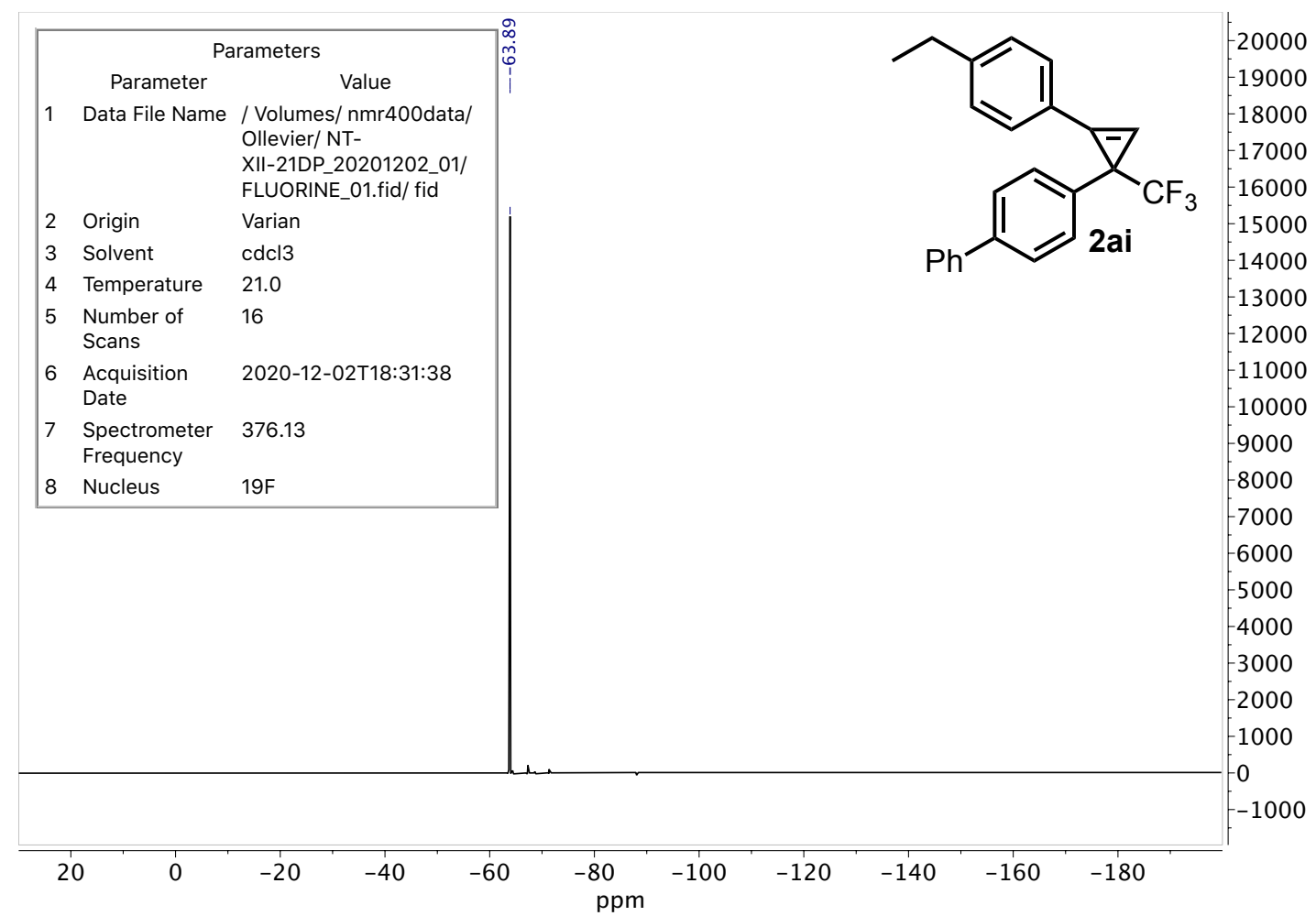

\section{4-(2-(4-Bromophenyl)-1-(trifluoromethyl)cycloprop-2-en-1-yl)-1,1'-biphenyl 2aj}

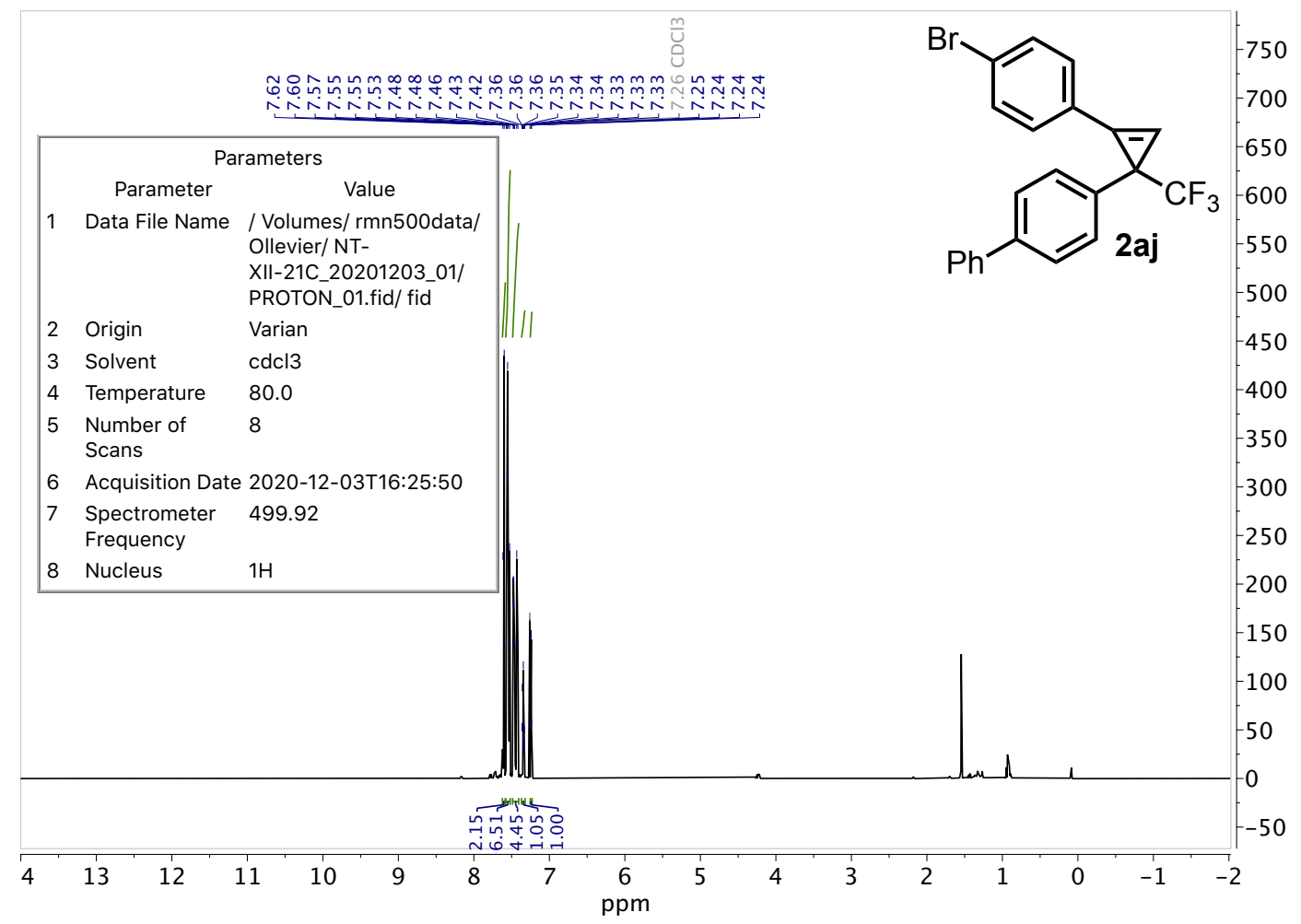




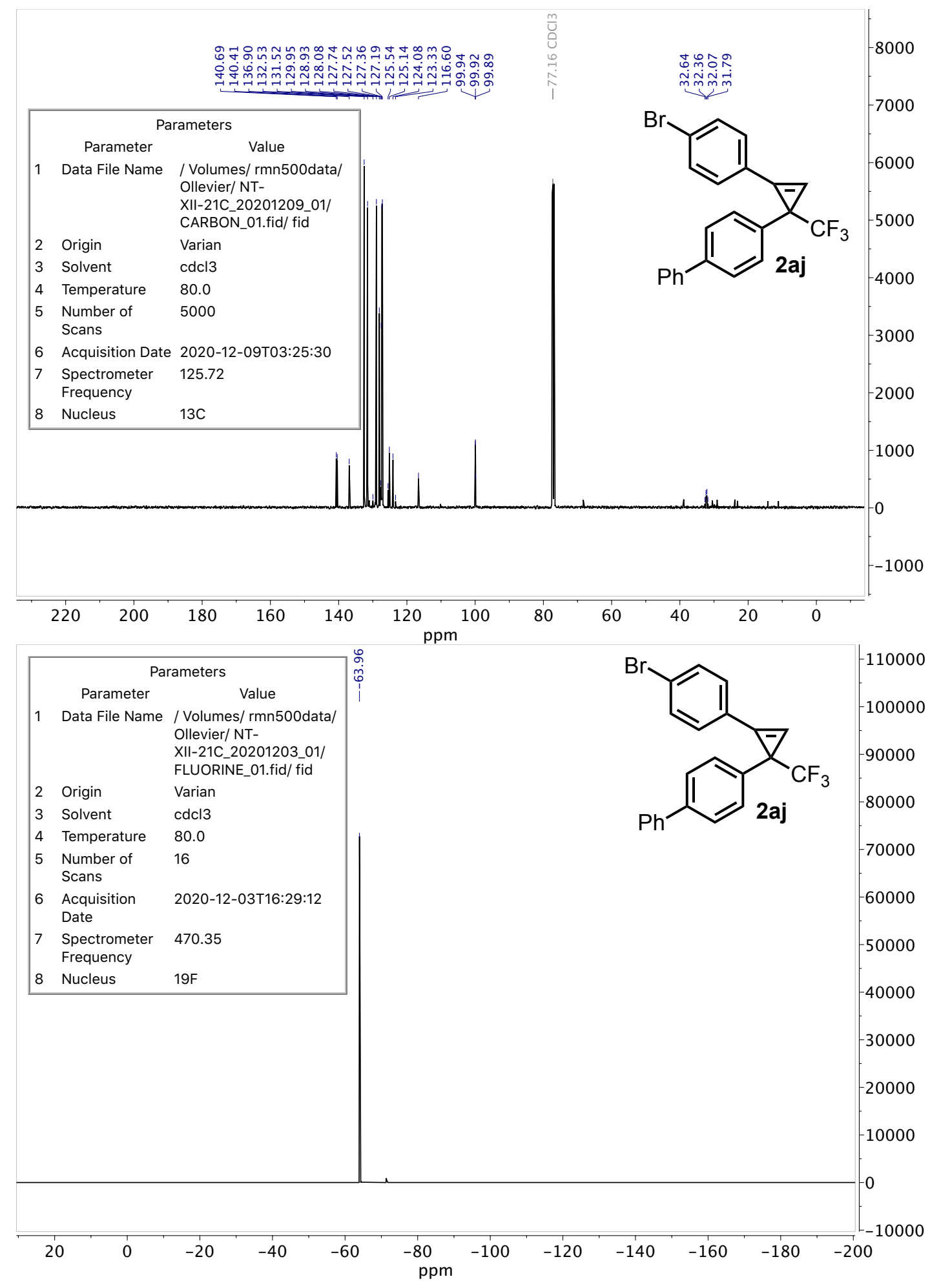


(3-([1,1'-Biphenyl]-4-yl)-3-(trifluoromethyl)cycloprop-1-en-1-yl)trimethylsilane 2ak

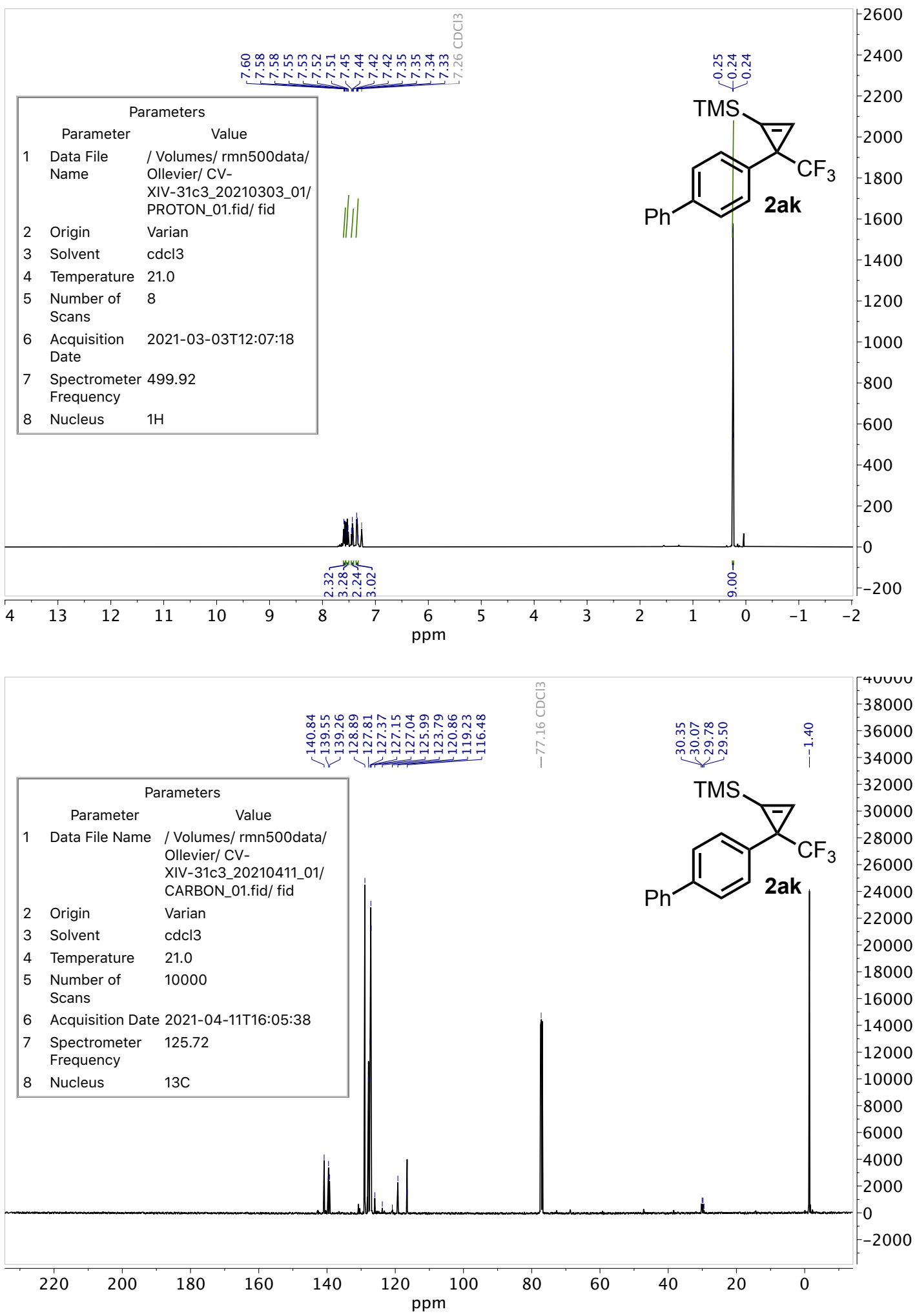




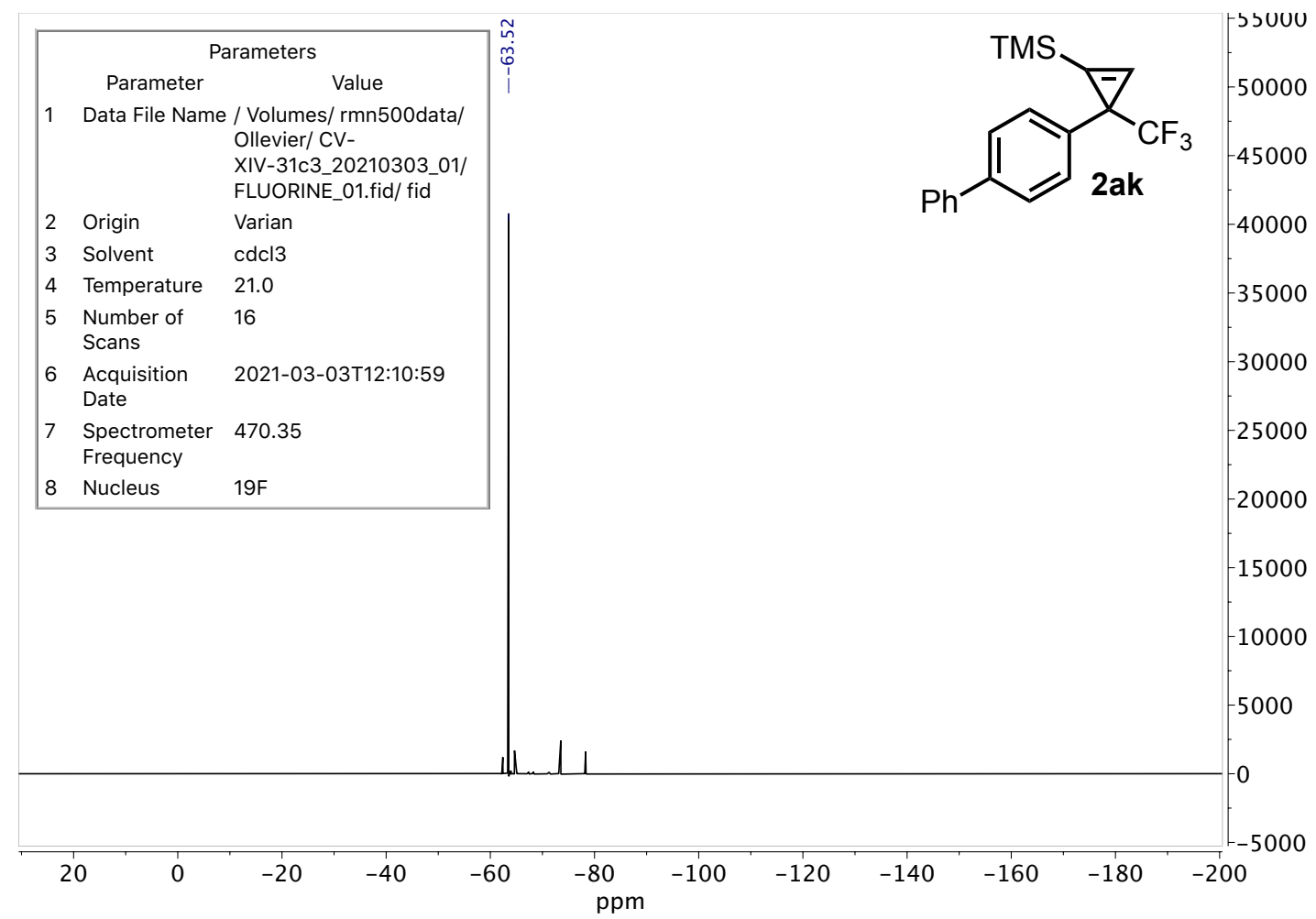

\section{4-(2-(3-Ethynylphenyl)-1-(trifluoromethyl)cycloprop-2-en-1-yl)-1,1'-biphenyl 2al}

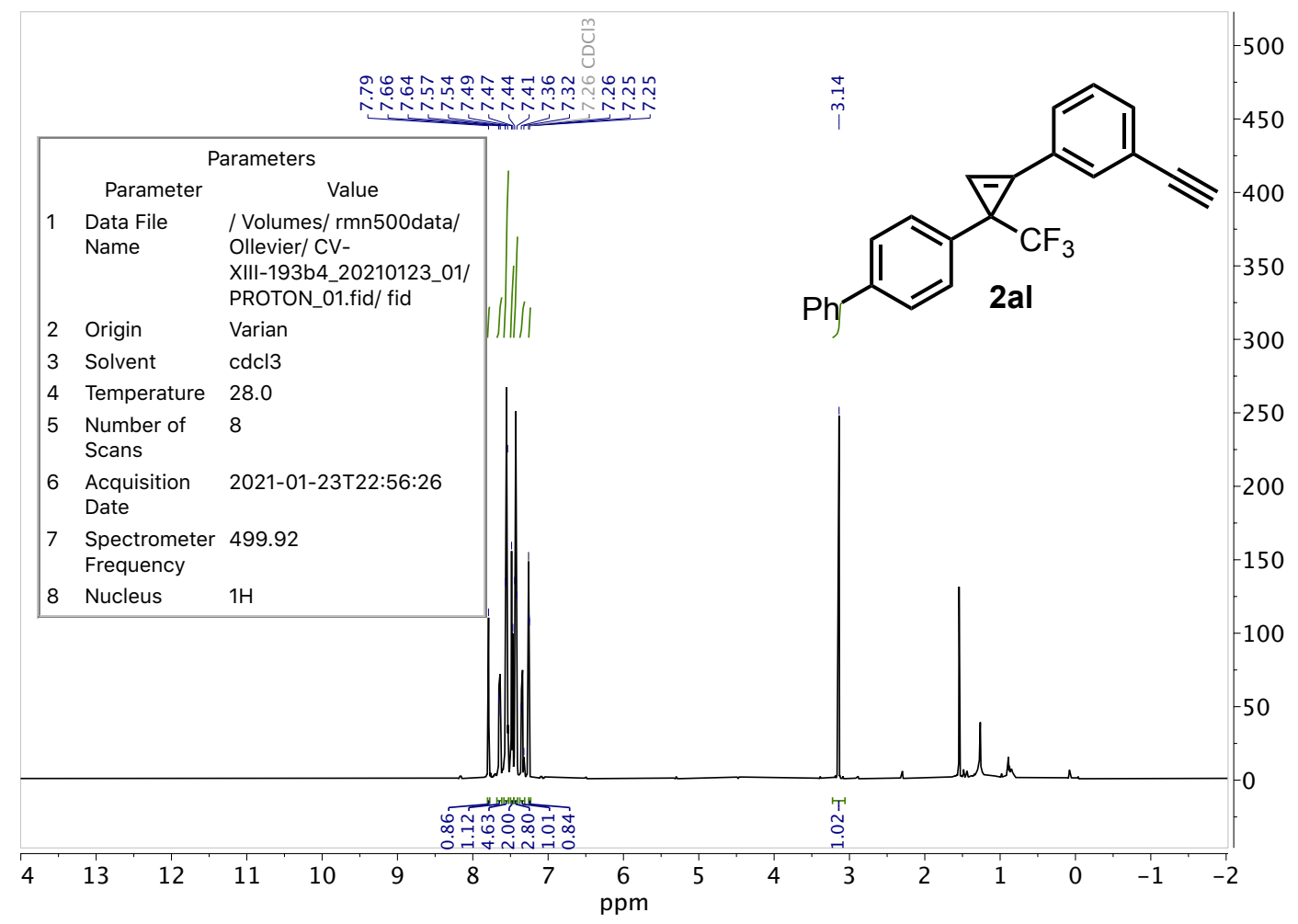




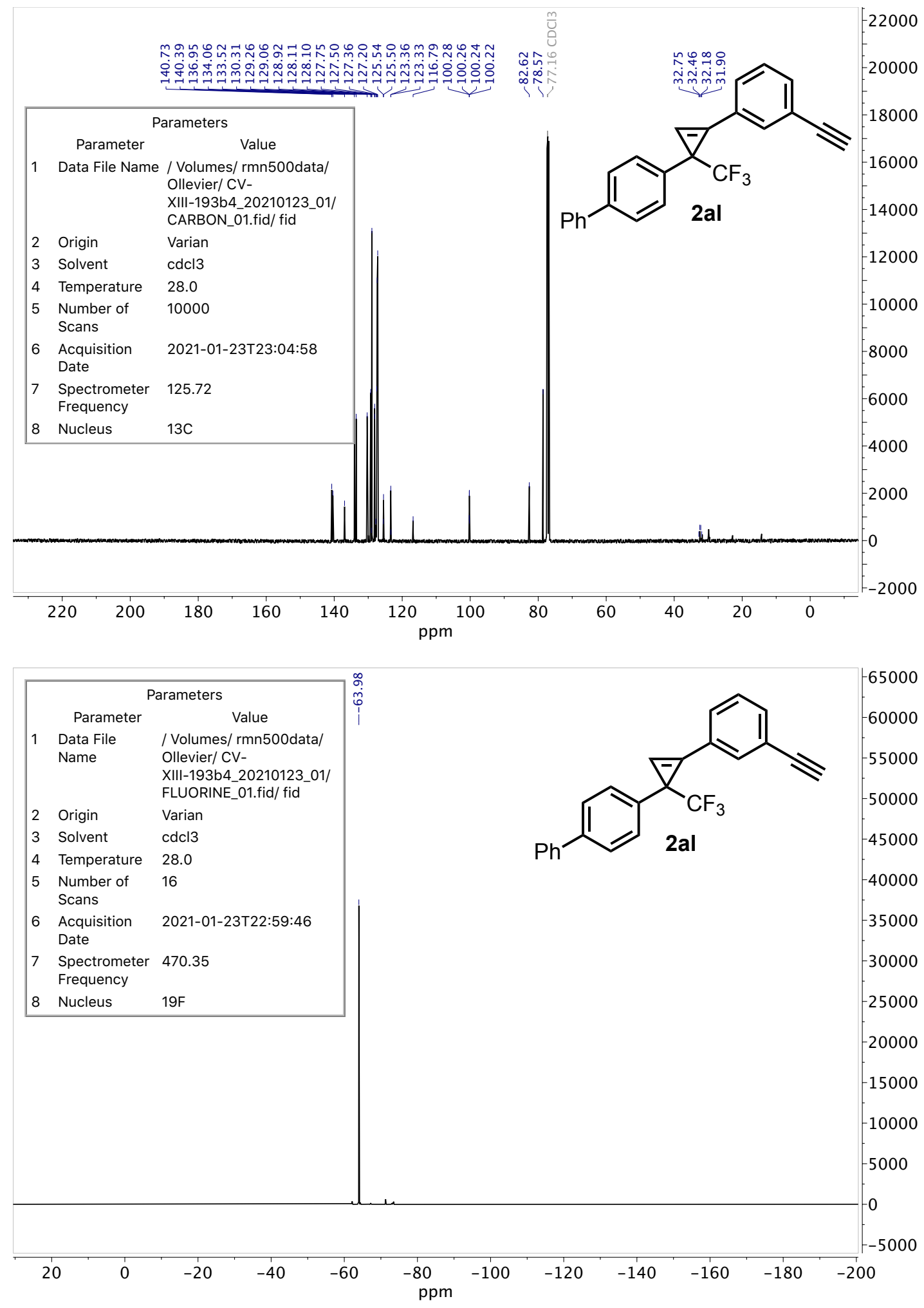




\section{4-(4-(3-Ethynylphenyl)-1,1,1-trifluorobut-3-yn-2-yl)-1,1'-biphenyl 2al'}
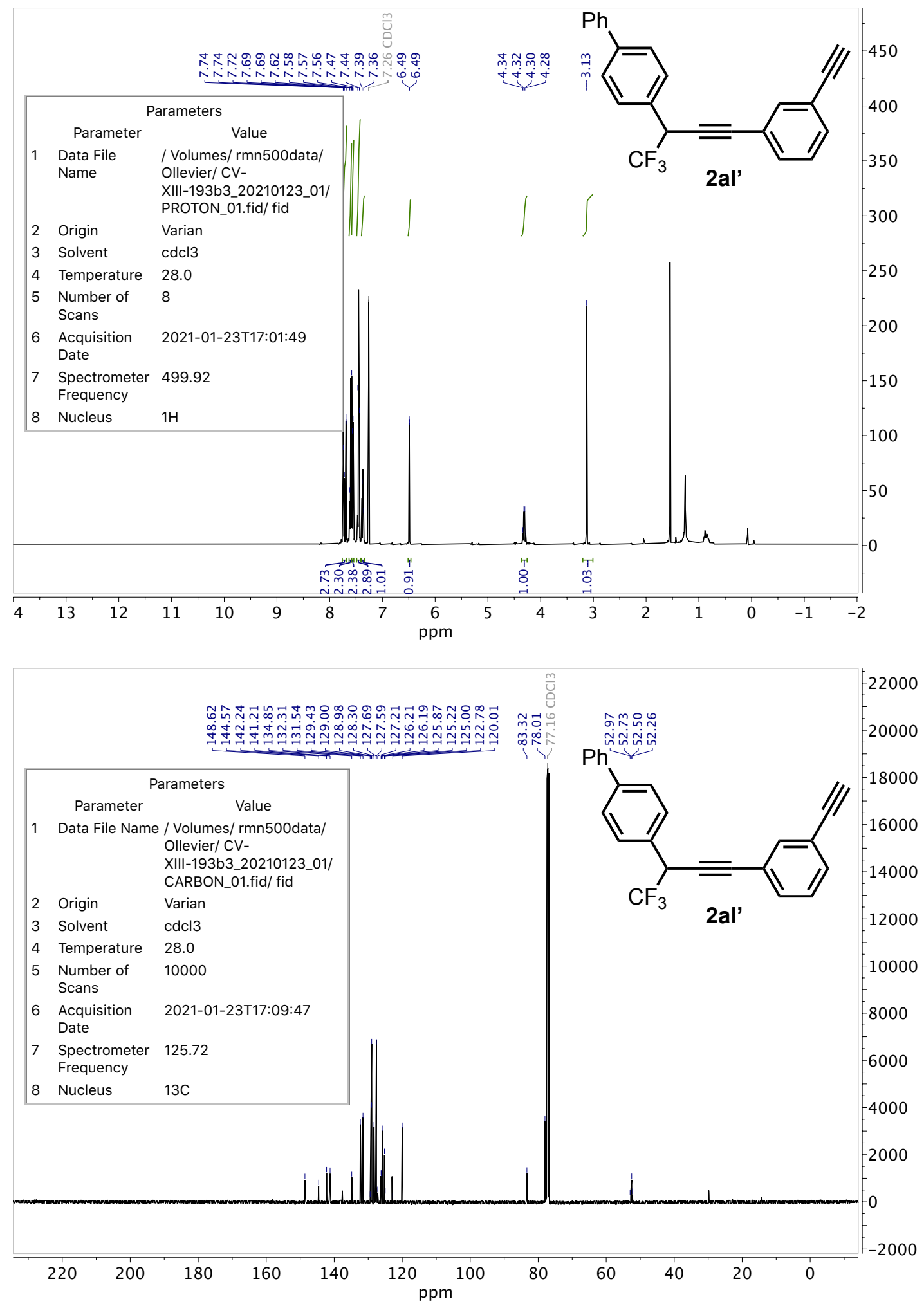

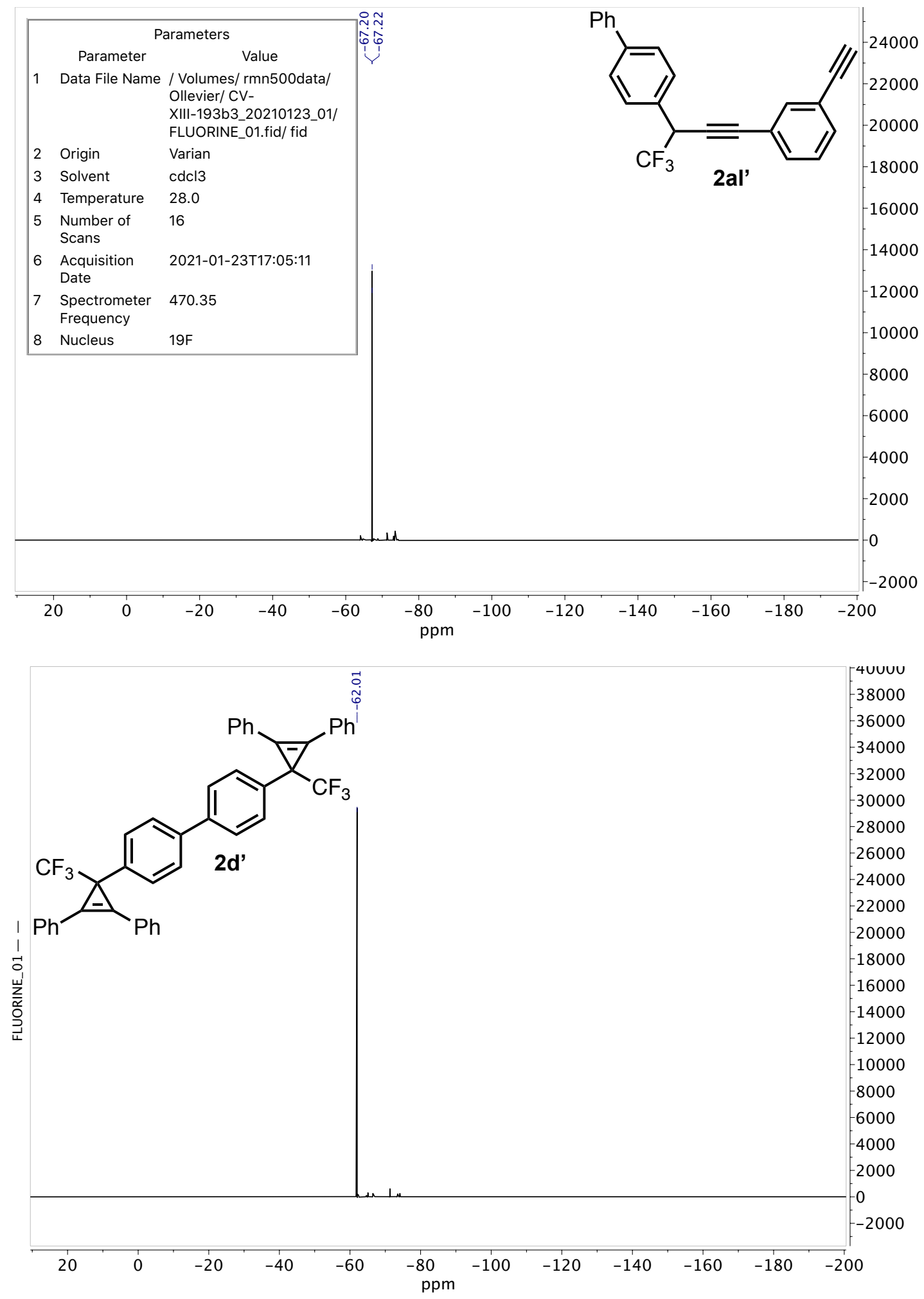


\section{2-(2,3-Diphenyl-1-(trifluoromethyl)cycloprop-2-en-1-yl)naphthalene 2b}
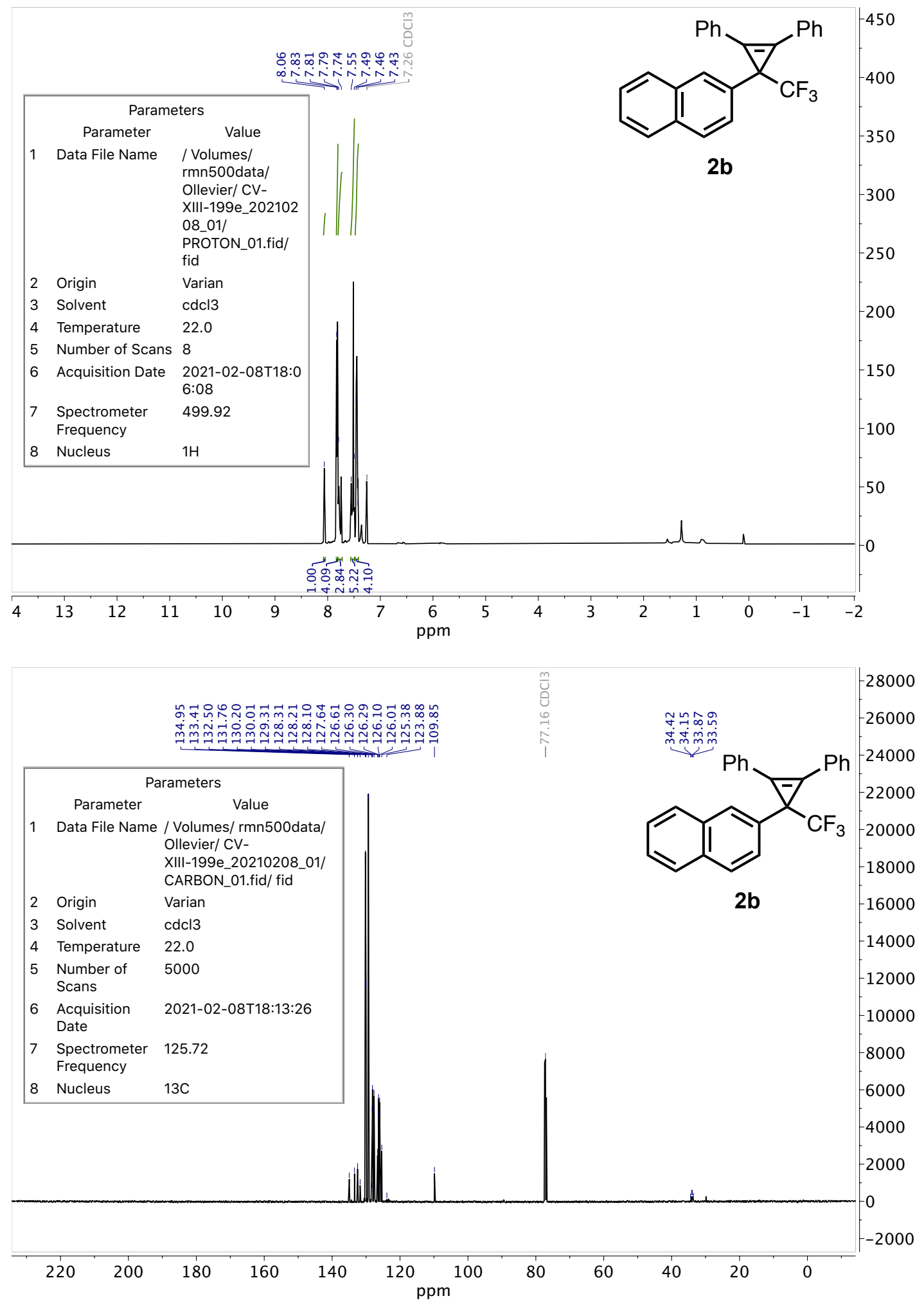


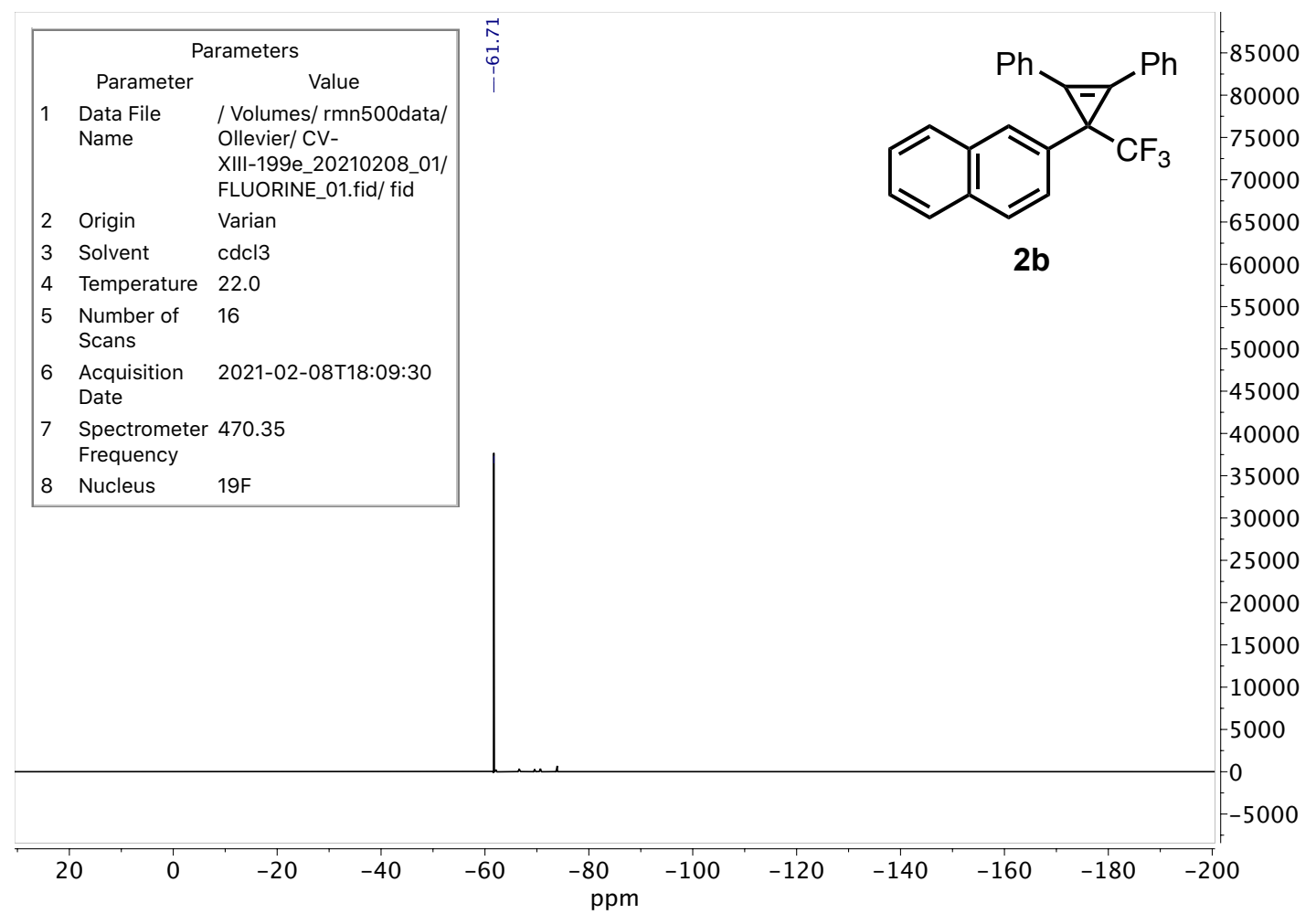

\section{(3-(4-(Benzyloxy)phenyl)-3-(trifluoromethyl)cycloprop-1-ene-1,2-diyl)dibenzene 2c}

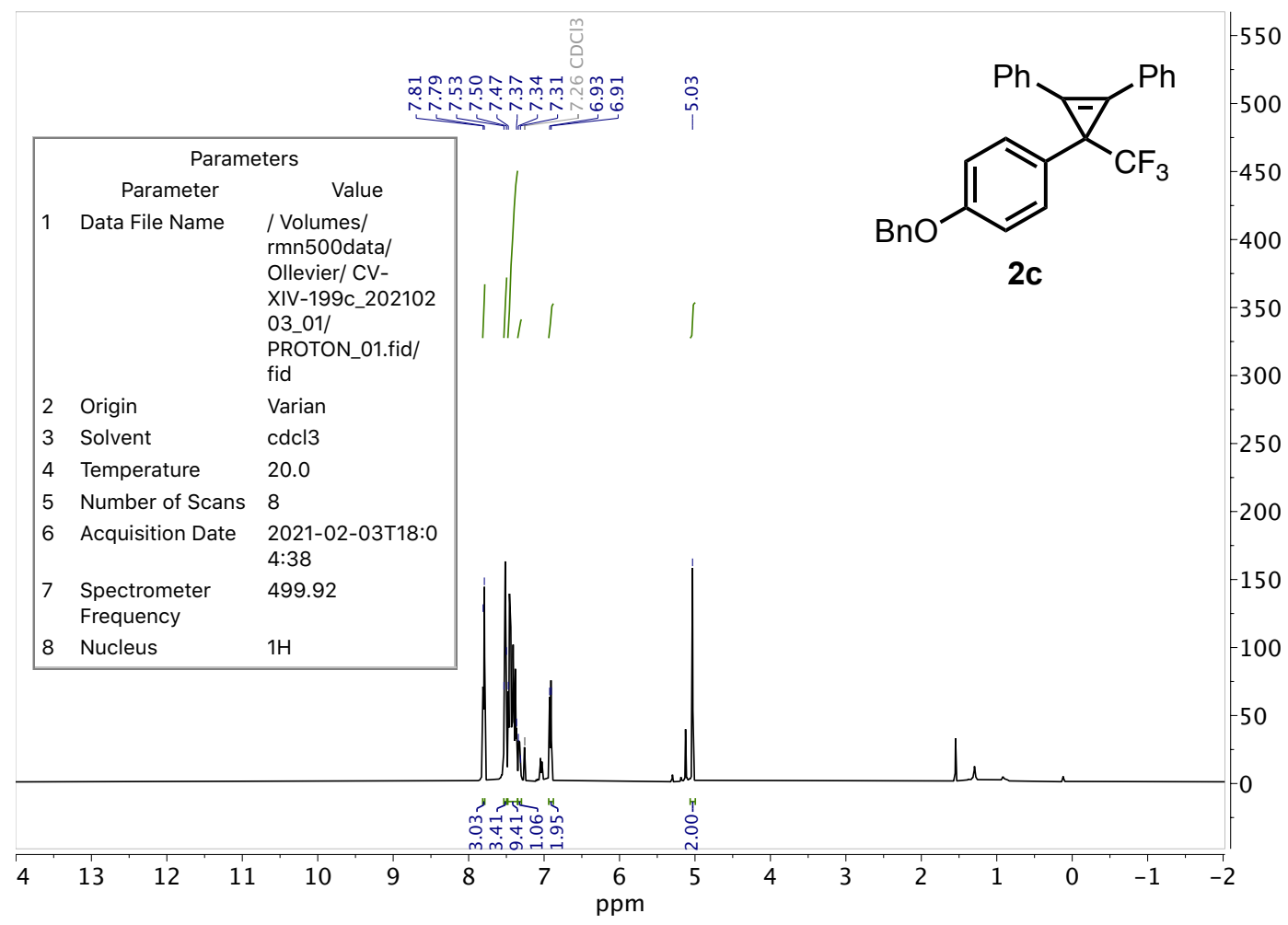



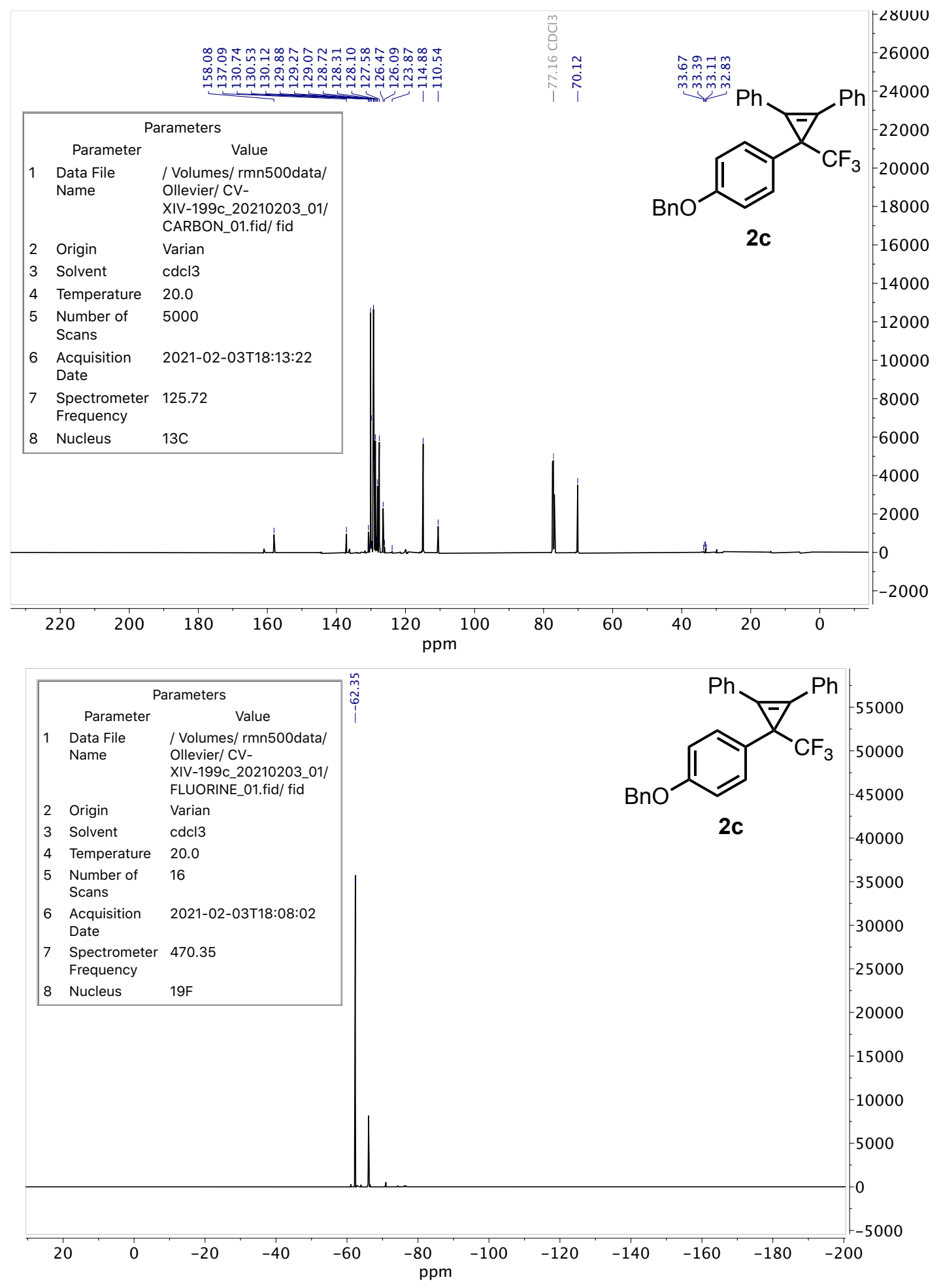


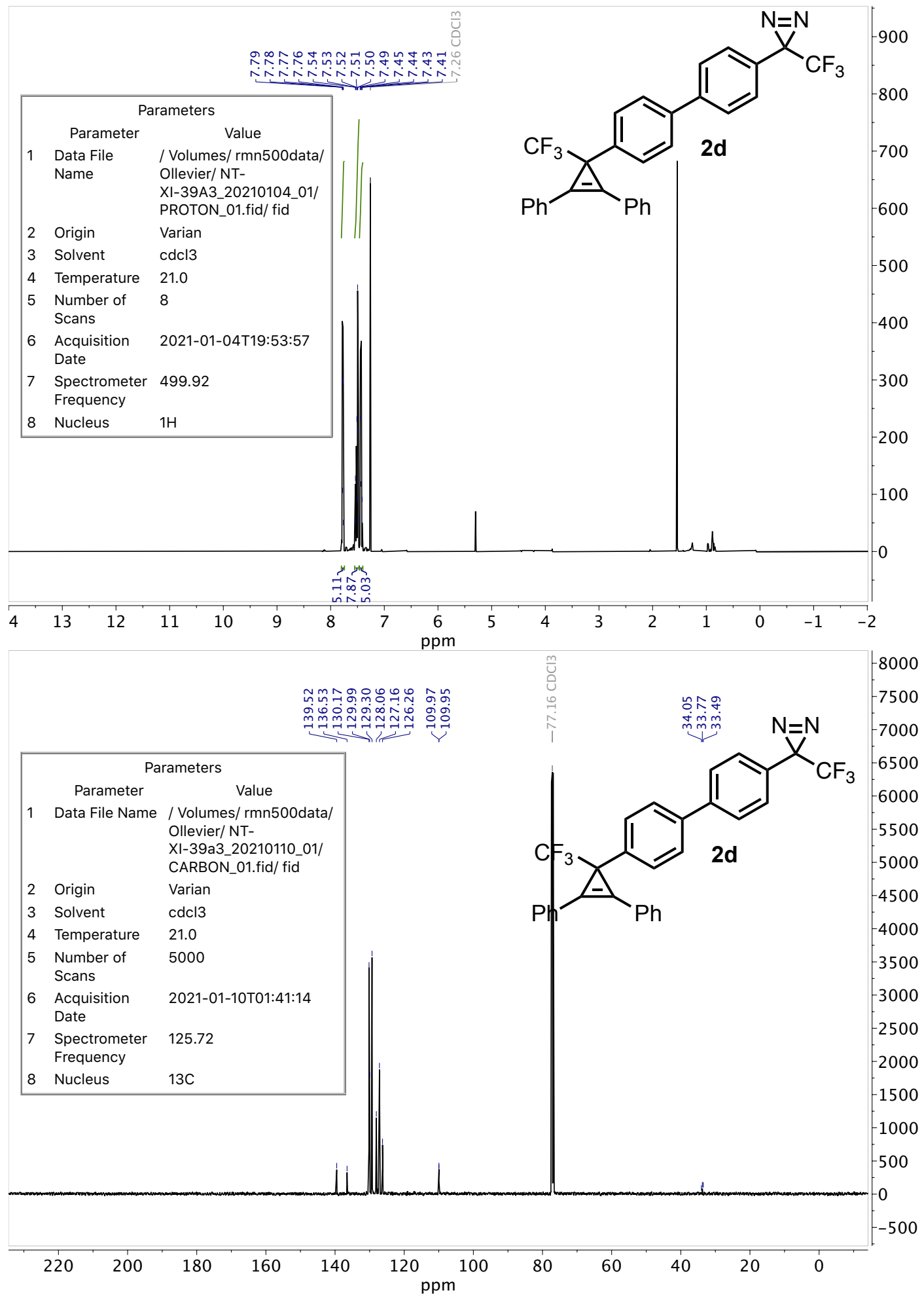




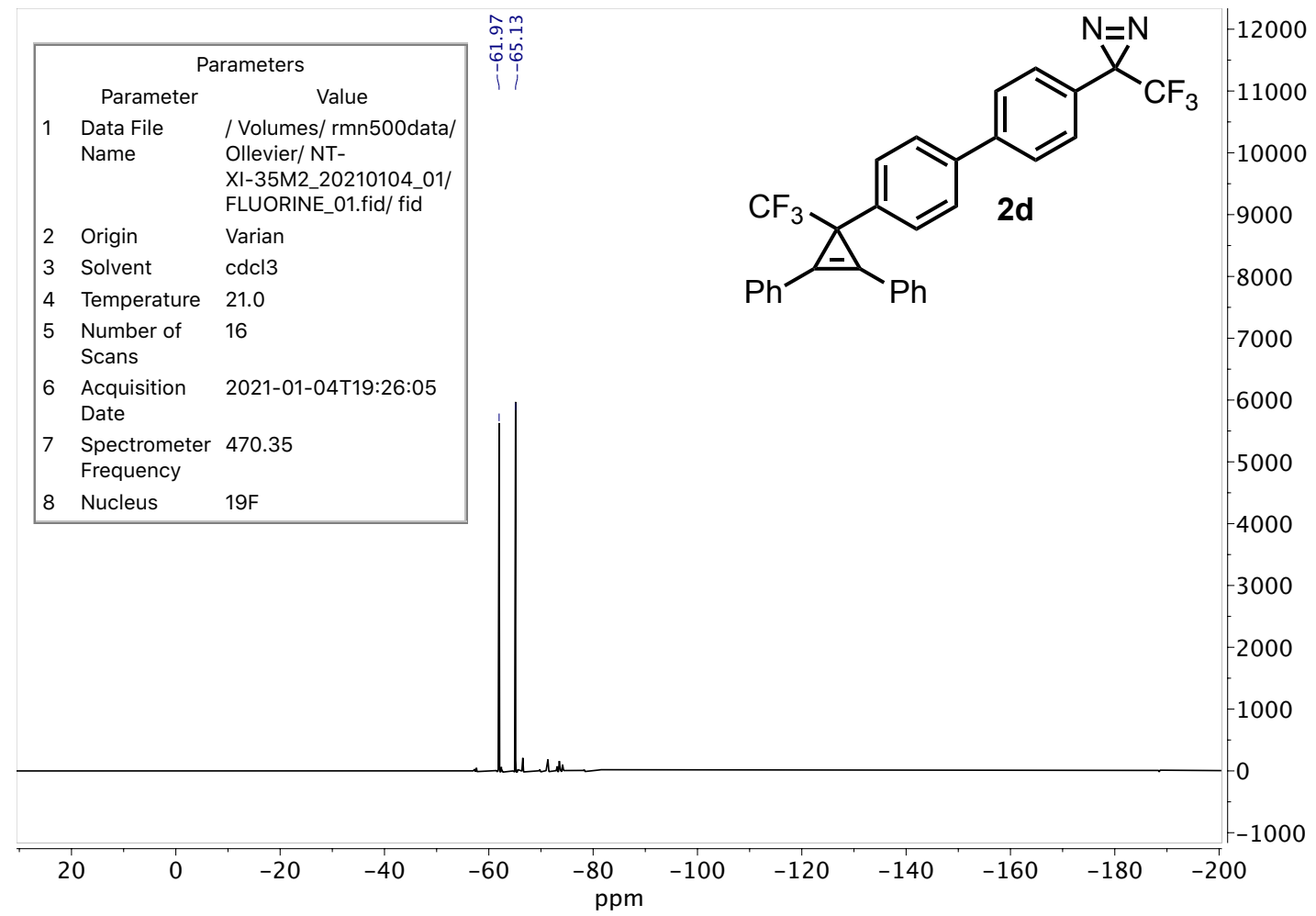

\section{4,4'-Bis(2,3-diphenyl-1-(trifluoromethyl)cycloprop-2-en-1-yl)-1,1'-biphenyl 2d'}

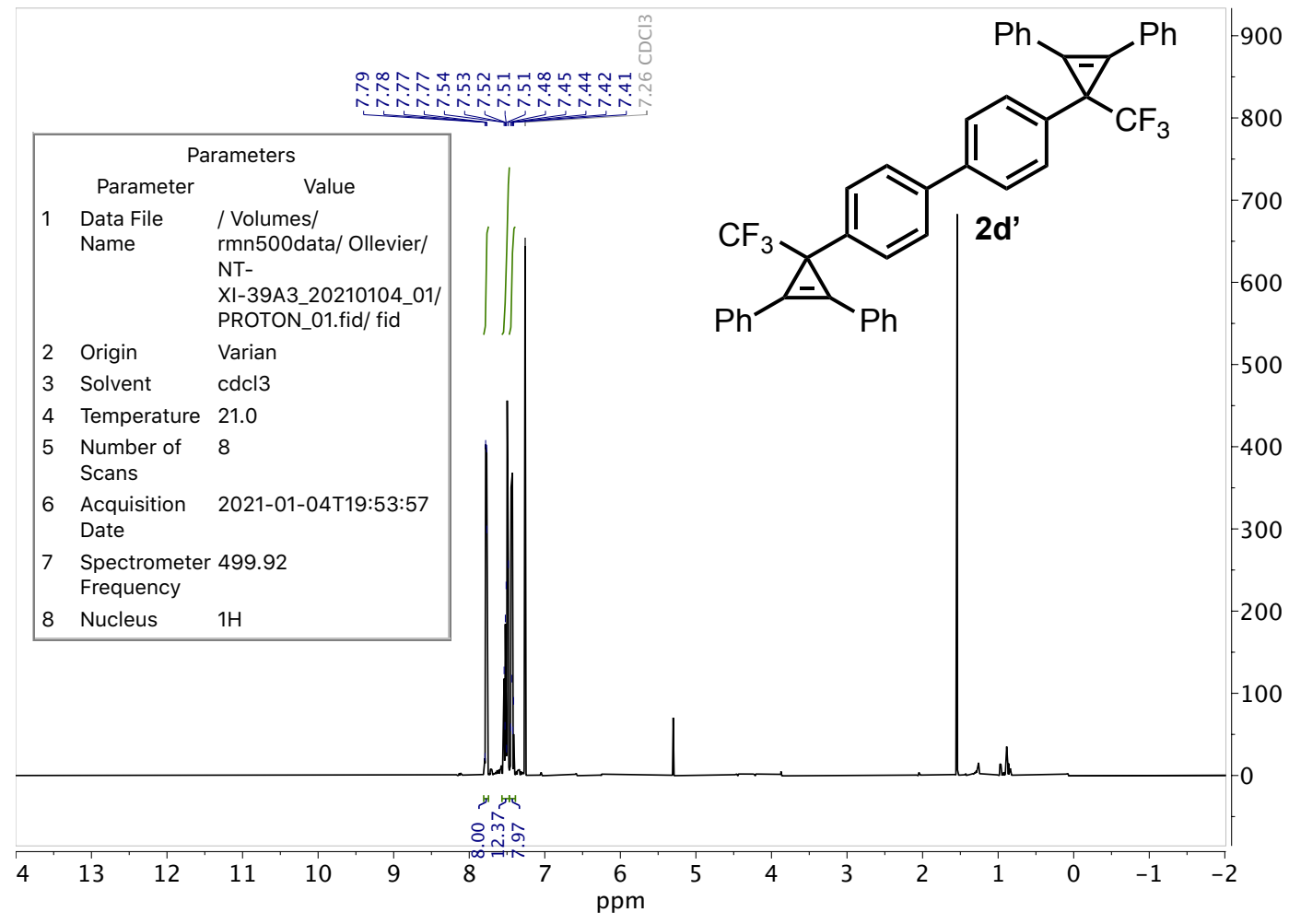




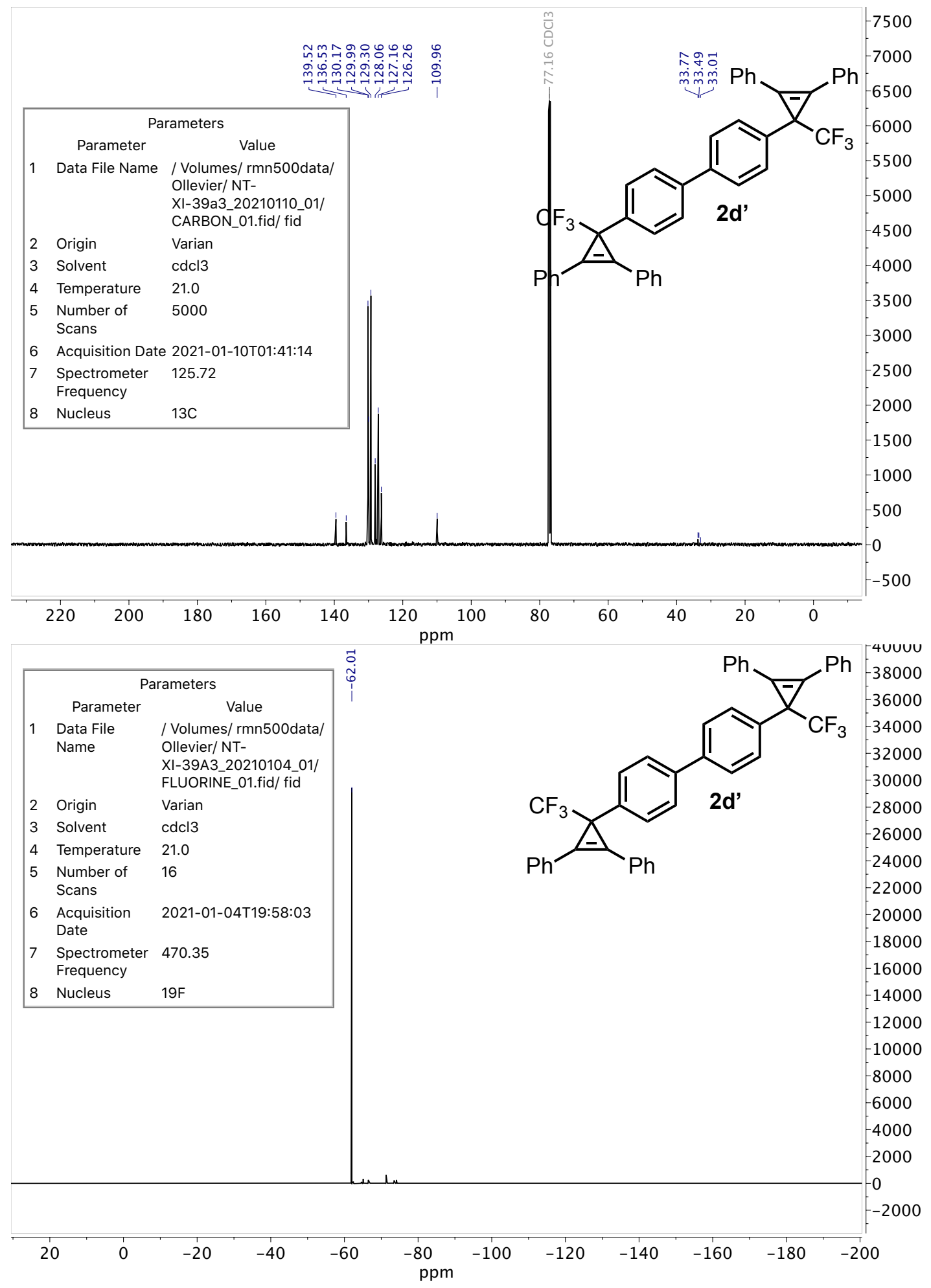


(3-(p-Tolyl)-3-(trifluoromethyl)cycloprop-1-ene-1,2-diyl)dibenzene 2e

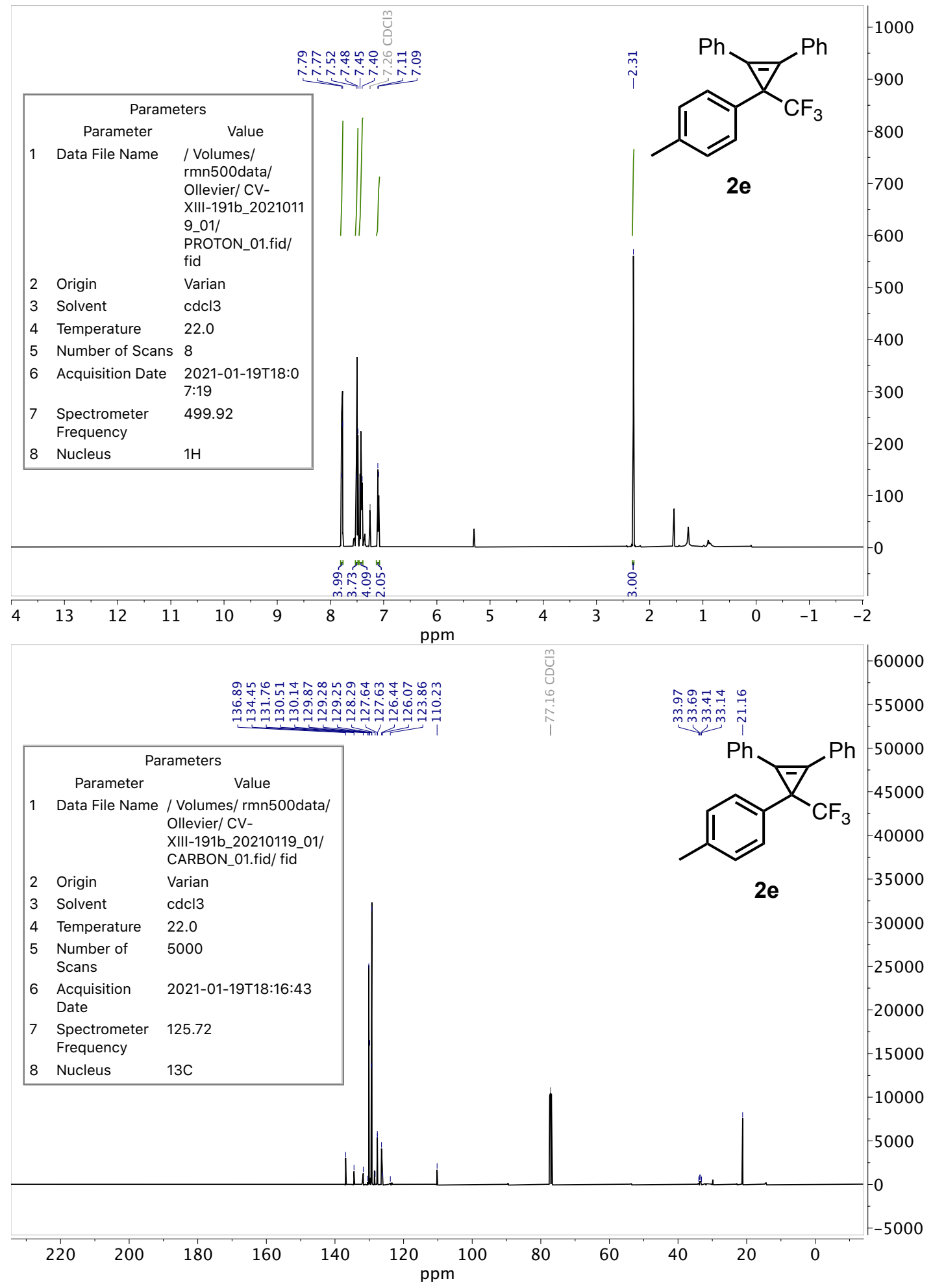




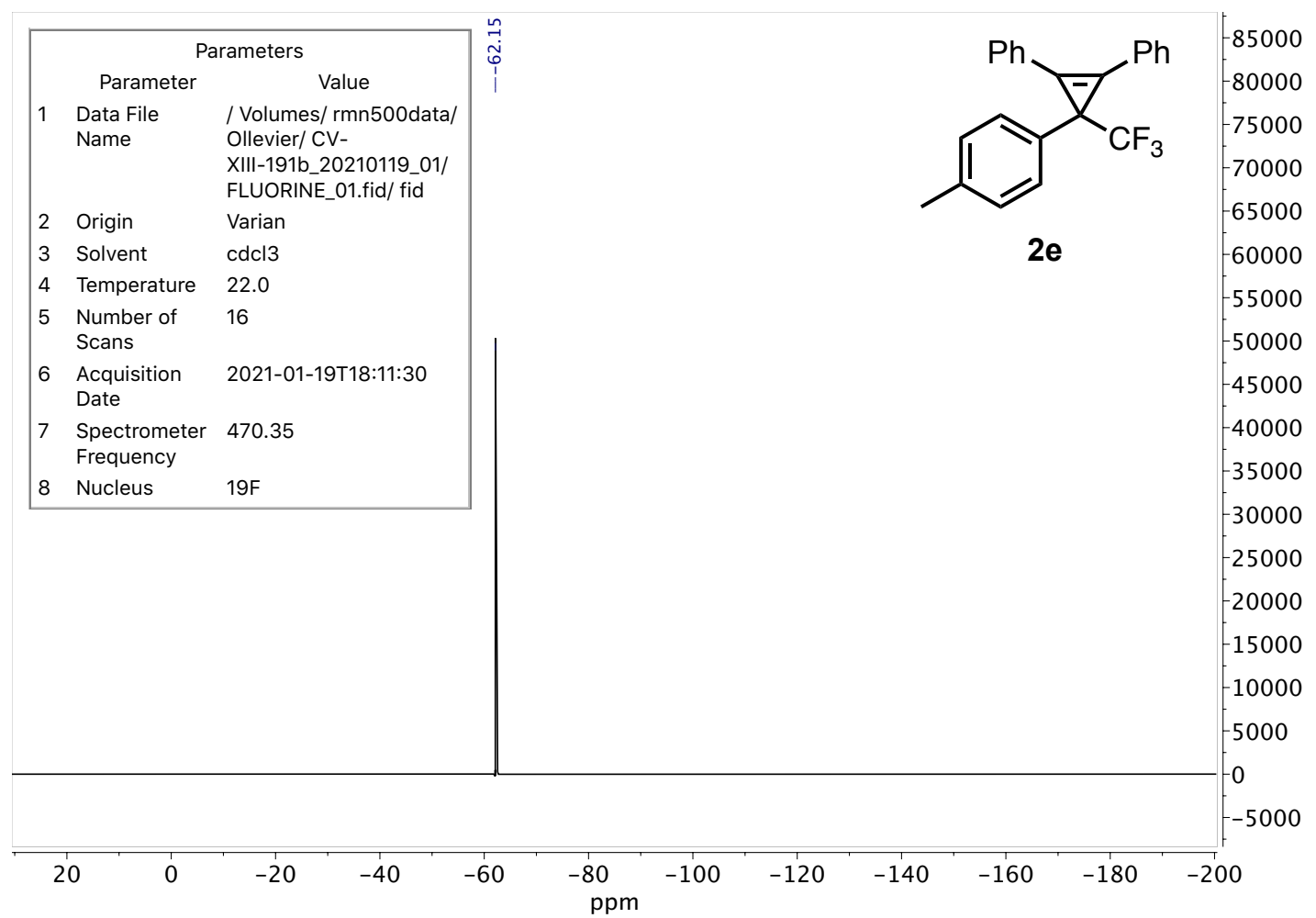

\section{(3-(4-(tert-Butyl)phenyl)-3-(trifluoromethyl)cycloprop-1-ene-1,2-diyl)dibenzene $2 f$}

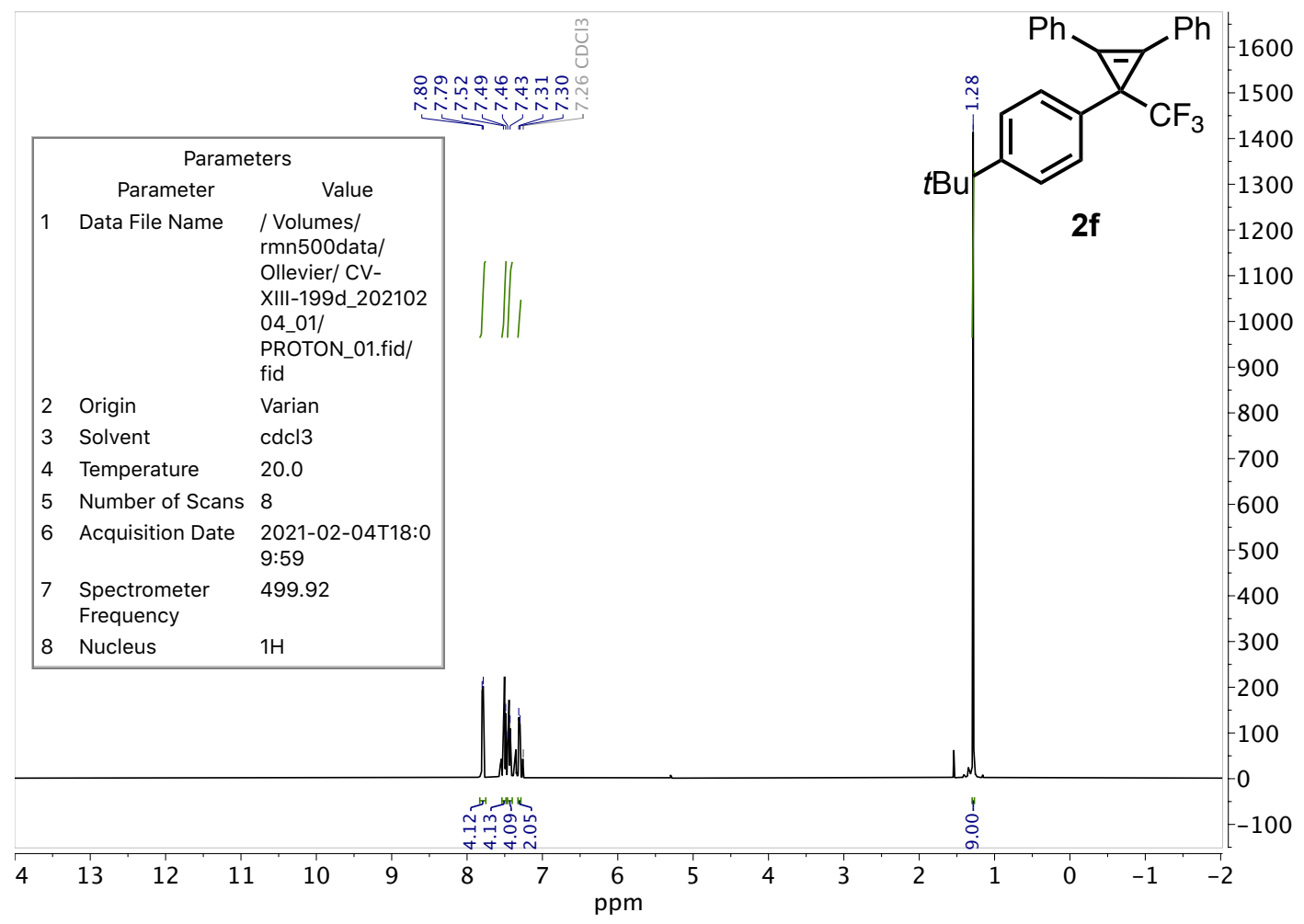




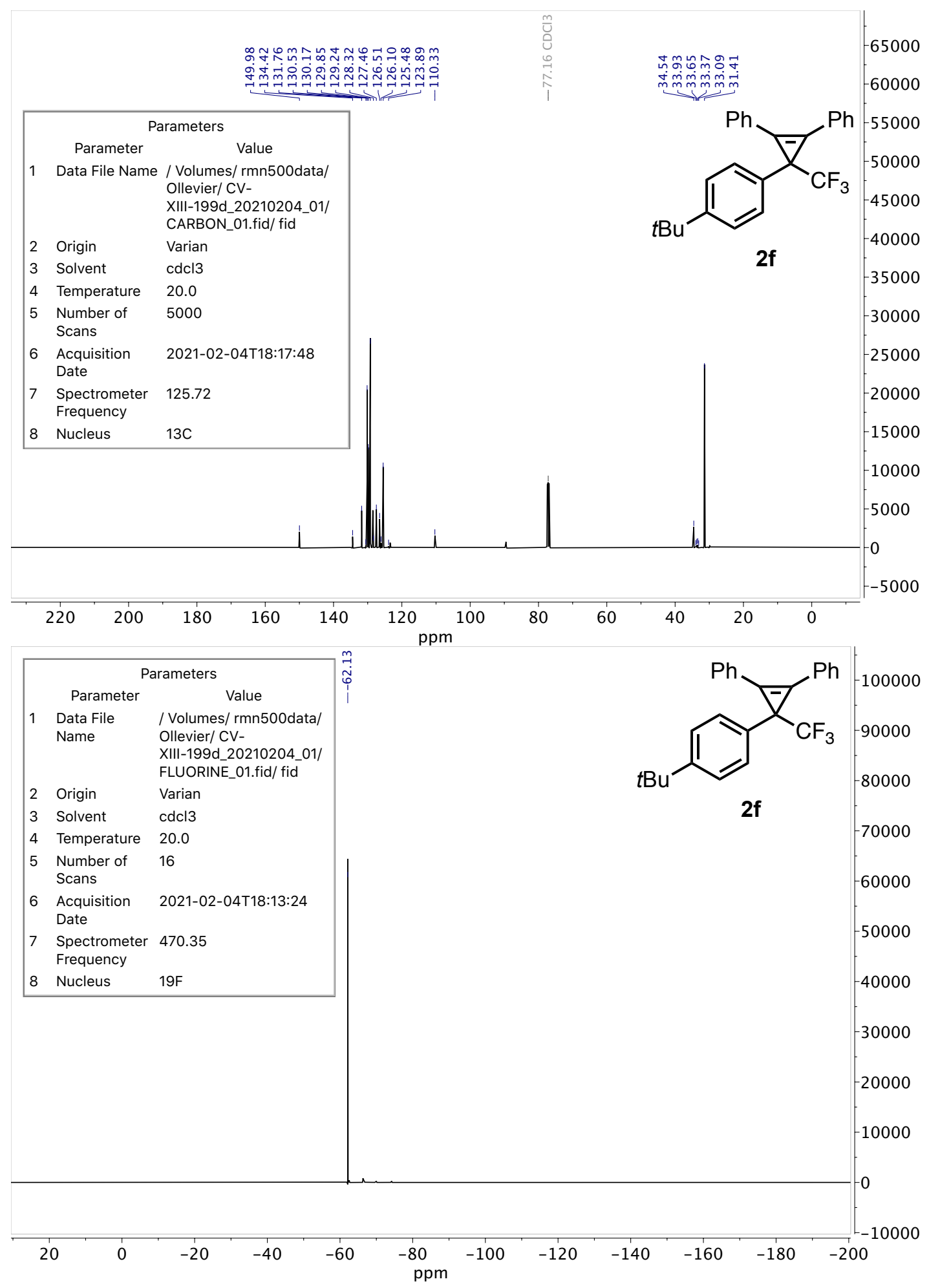


(3-(4-Methoxyphenyl)-3-(trifluoromethyl)cycloprop-1-ene-1,2-diyl)dibenzene 2g

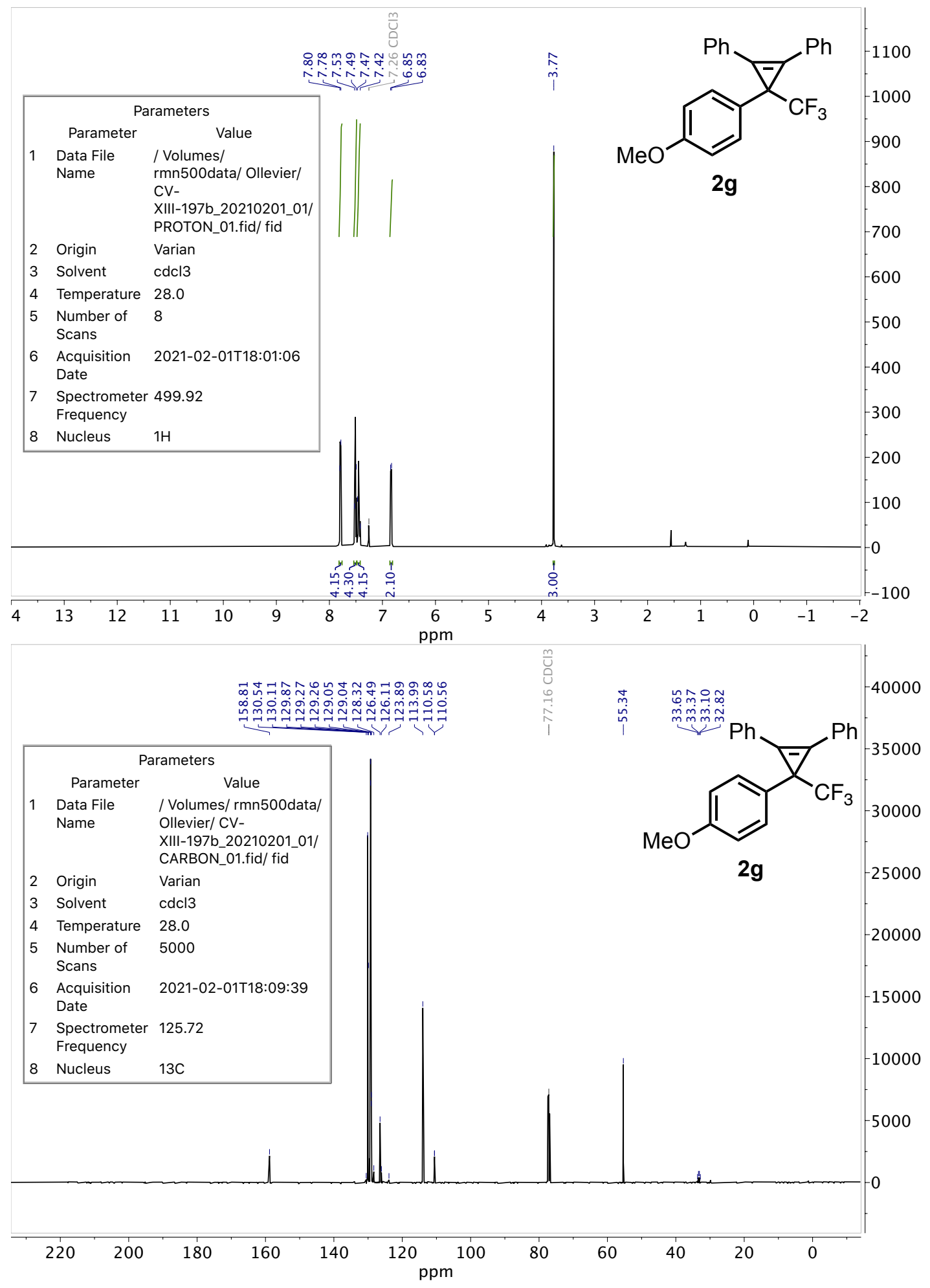




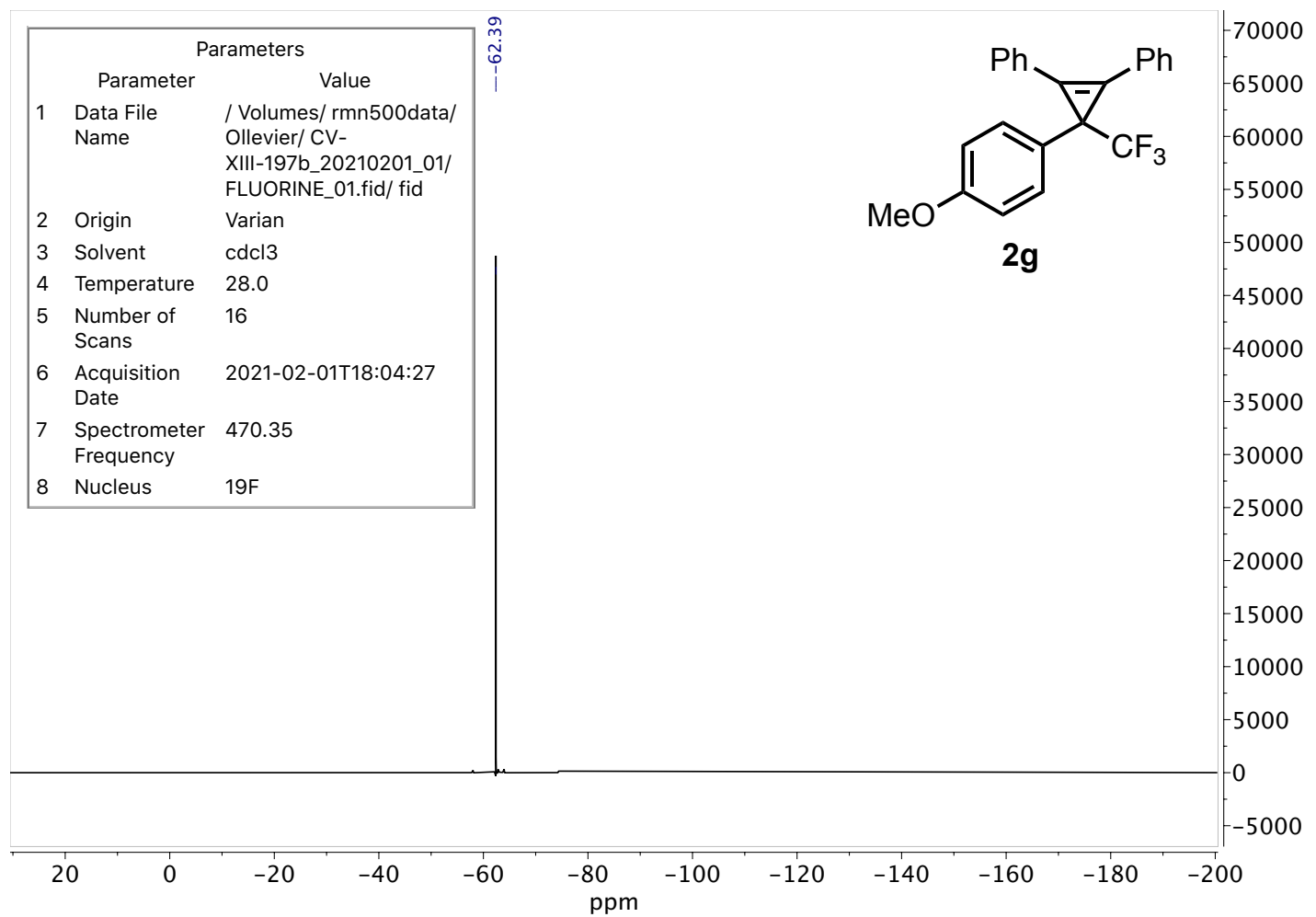

(3-(Trifluoromethyl)cycloprop-1-ene-1,2,3-triyl)tribenzene $2 \mathrm{~h}$

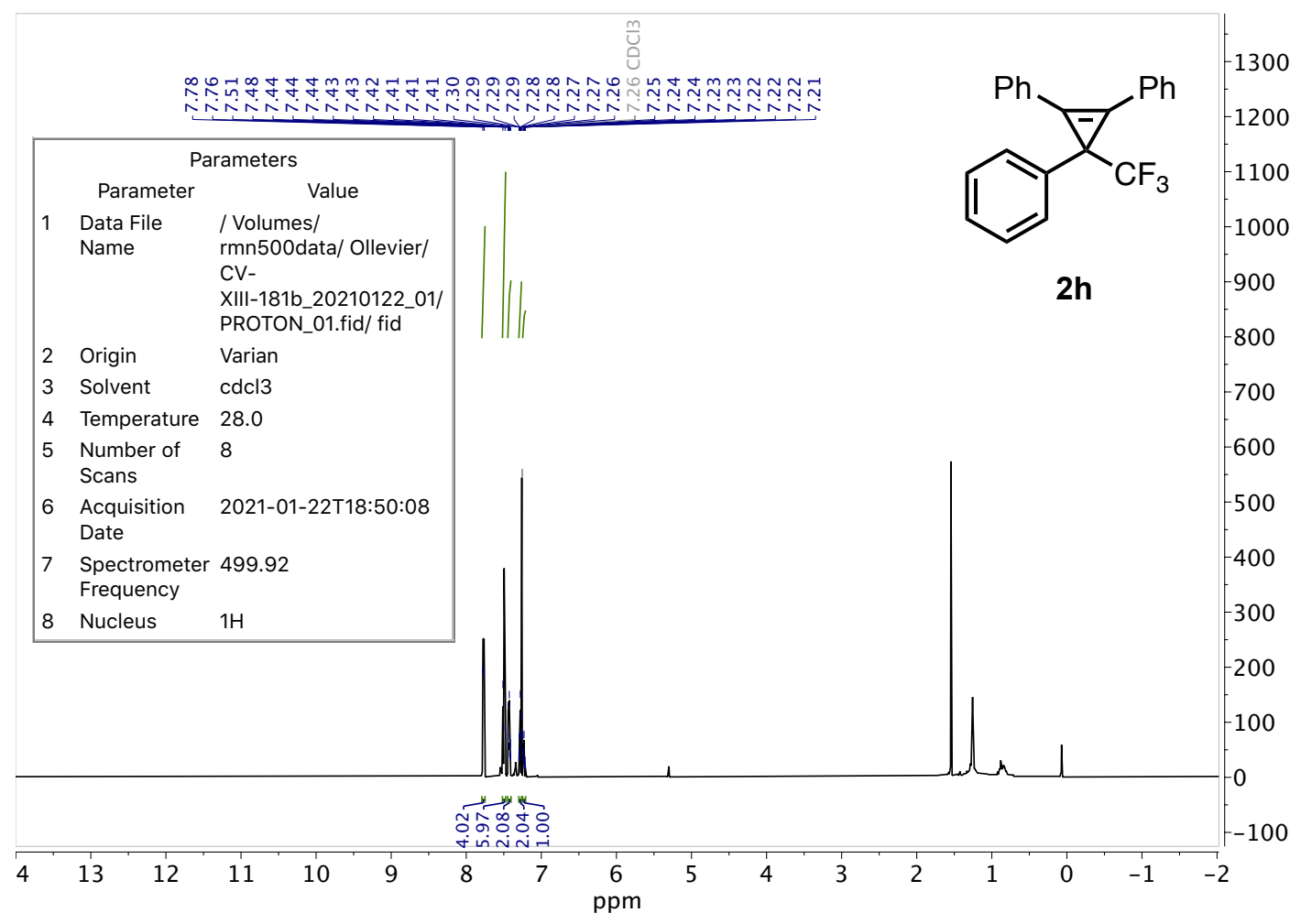




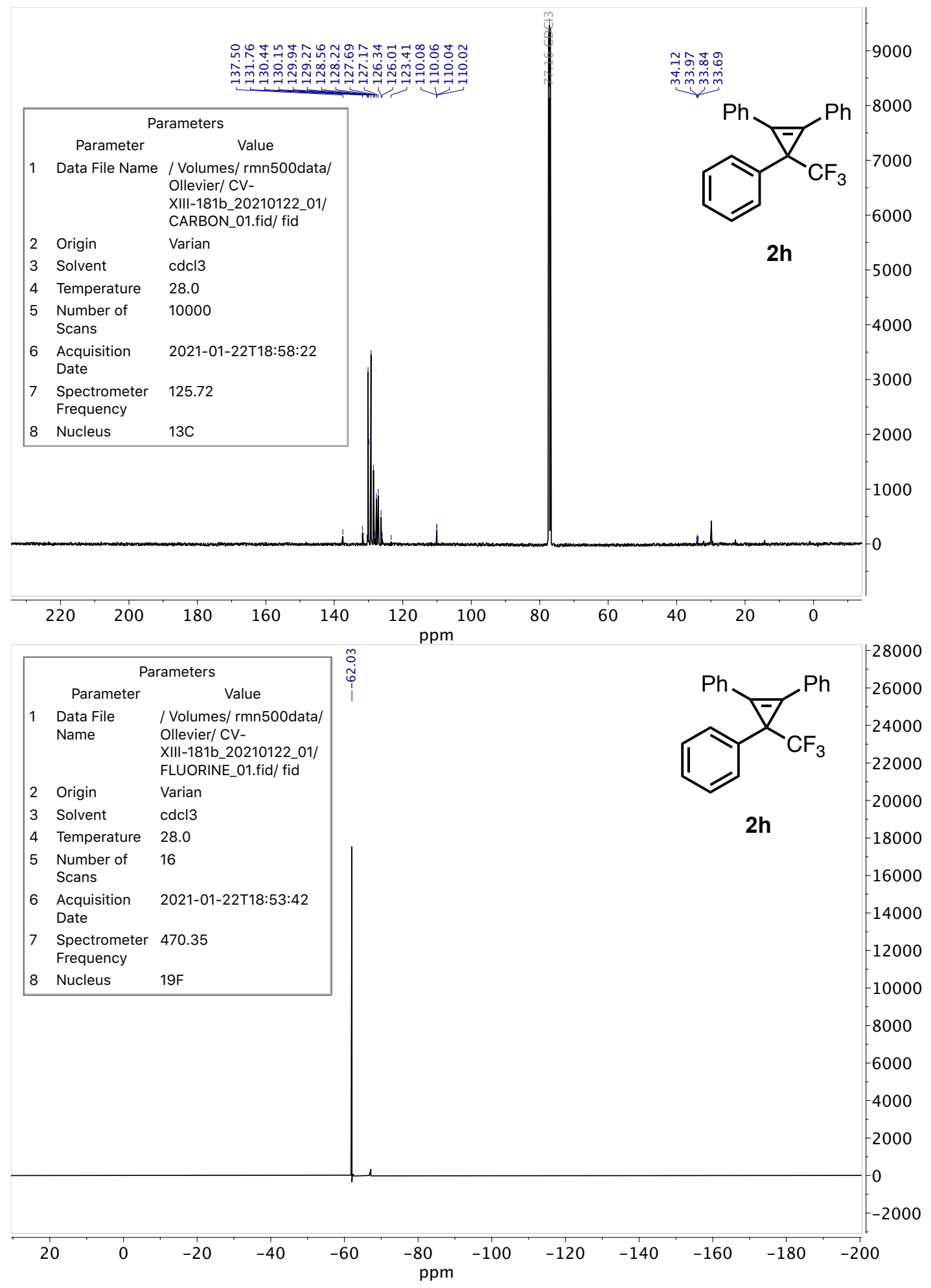


(3-(4-Fluorophenyl)-3-(trifluoromethyl)cycloprop-1-ene-1,2-diyl)dibenzene 2i

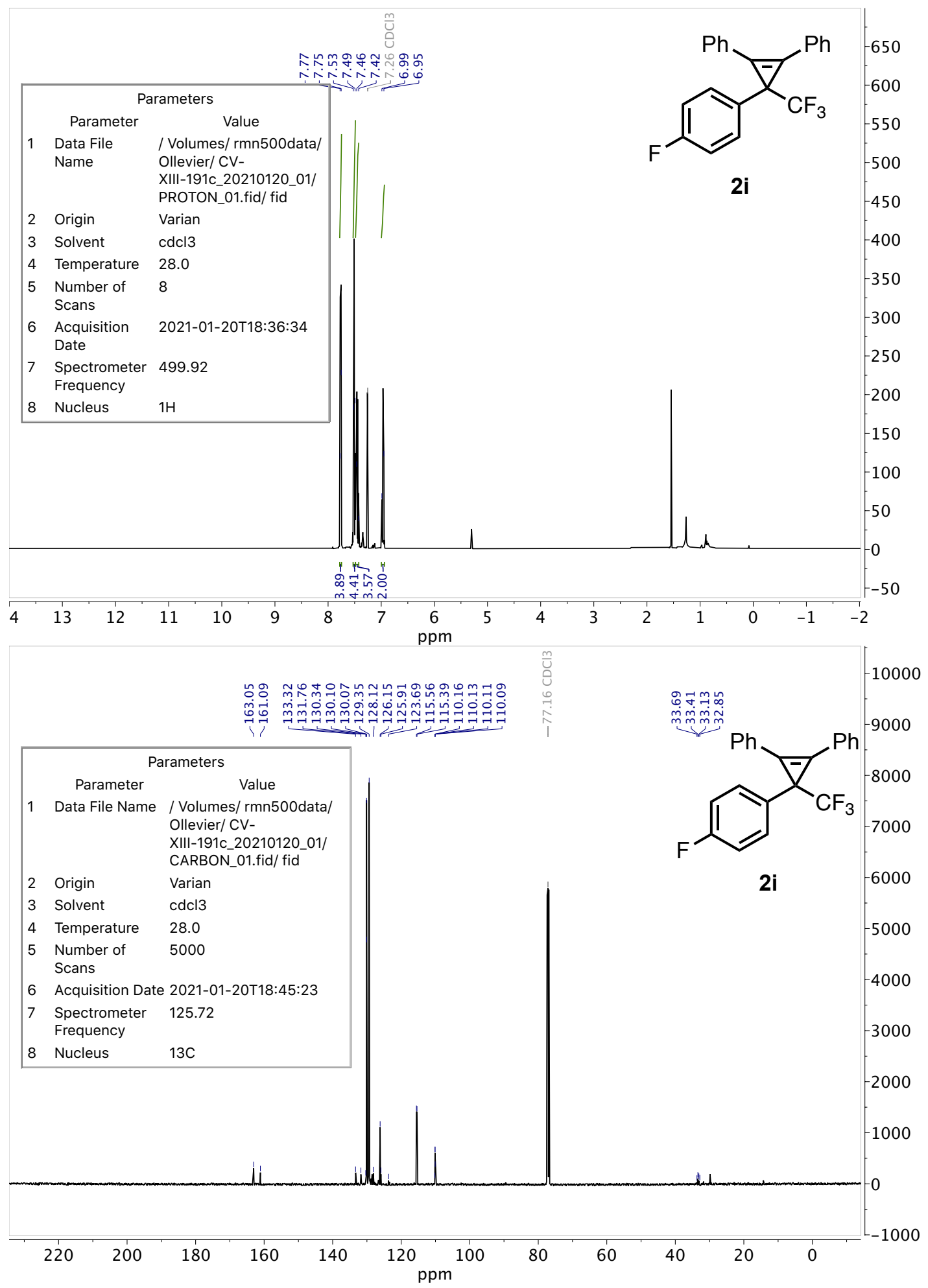




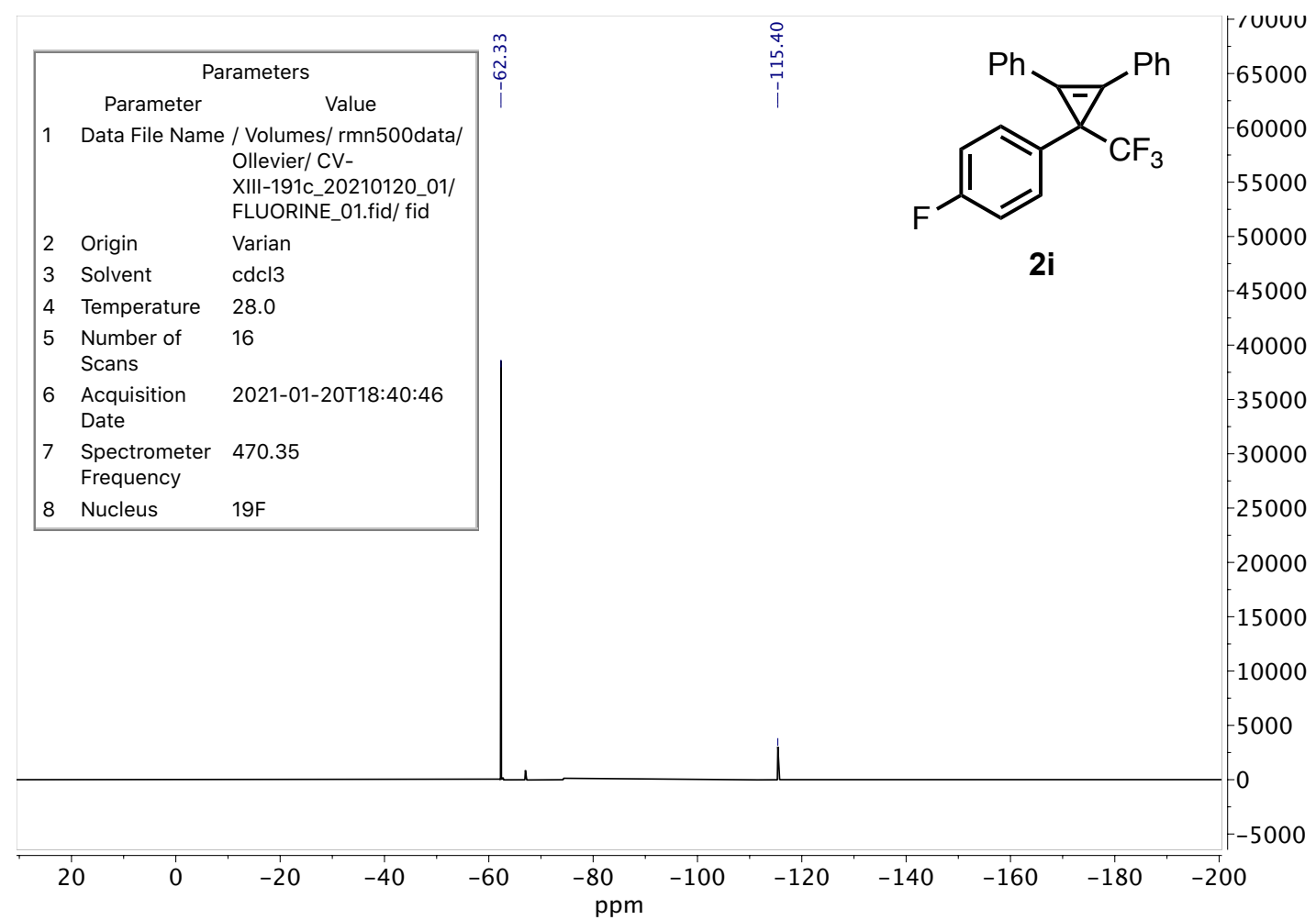

\section{(3-(4-Bromophenyl)-3-(trifluoromethyl)cycloprop-1-ene-1,2-diyl)dibenzene 2j}

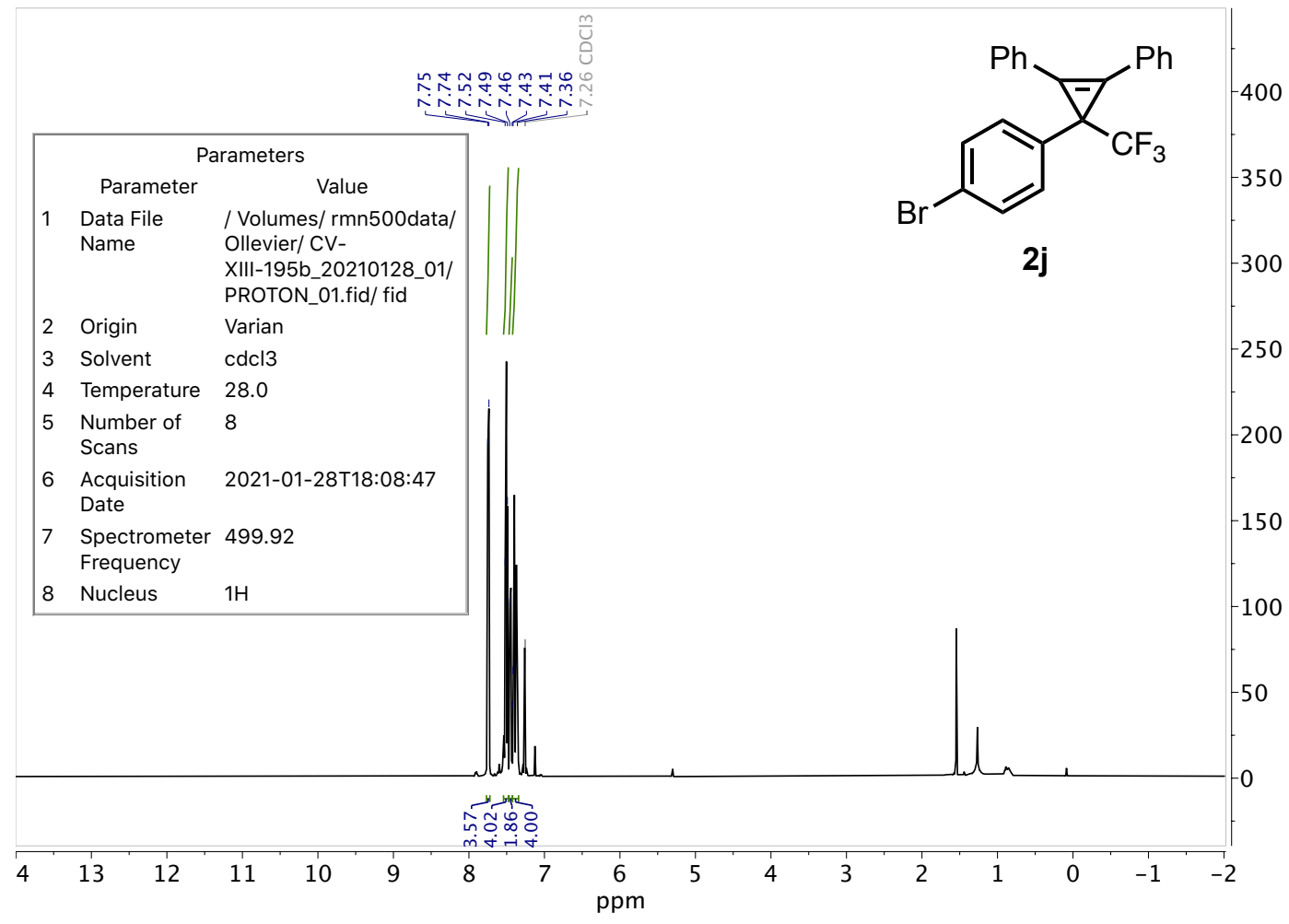




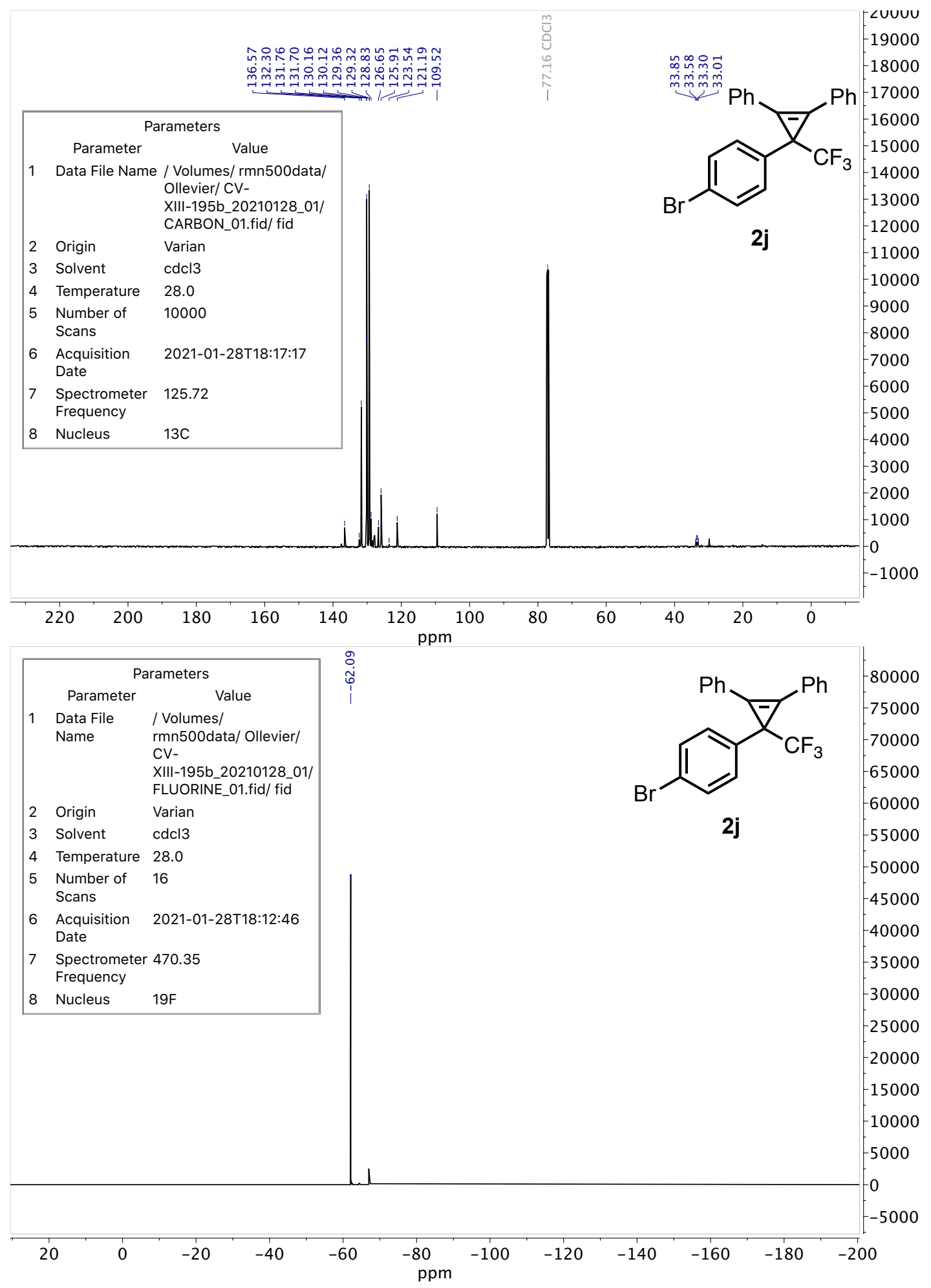




\section{(3-(3-Bromophenyl)-3-(trifluoromethyl)cycloprop-1-ene-1,2-diyl)dibenzene 2k}

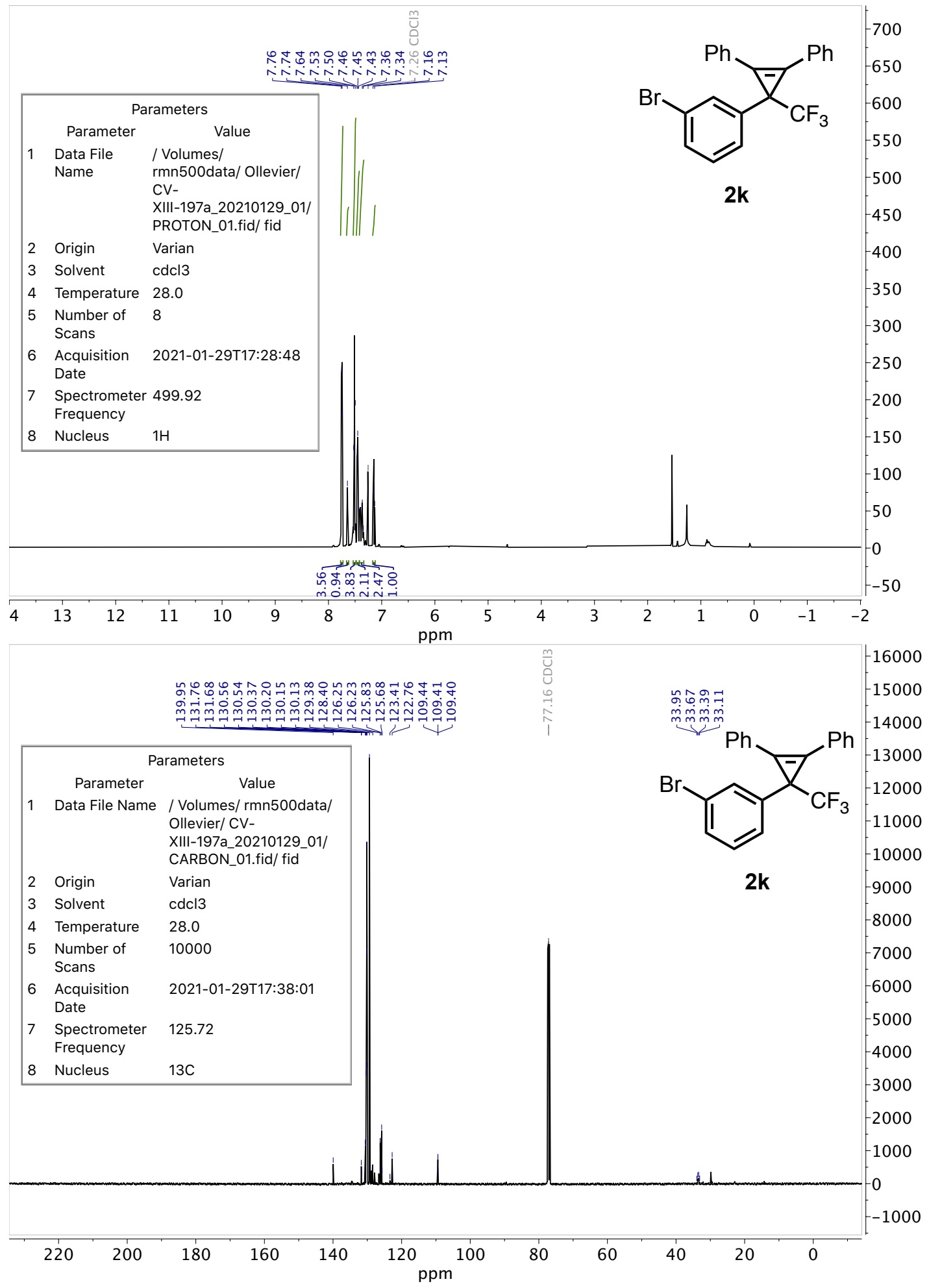




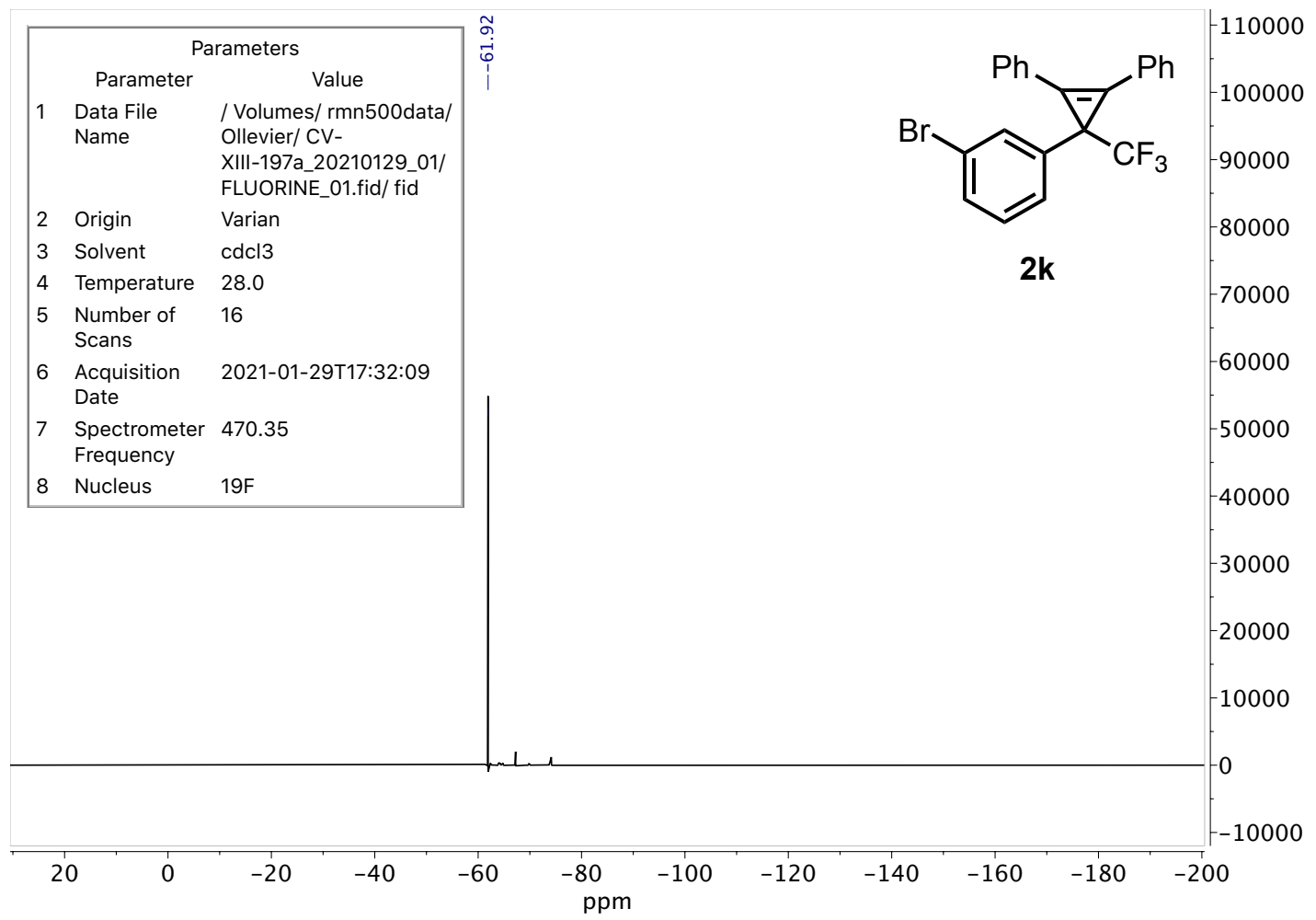

(3-(Trifluoromethyl)-3-(4-(trifluoromethyl)phenyl)cycloprop-1-ene-1,2-diyl)dibenzene 2I

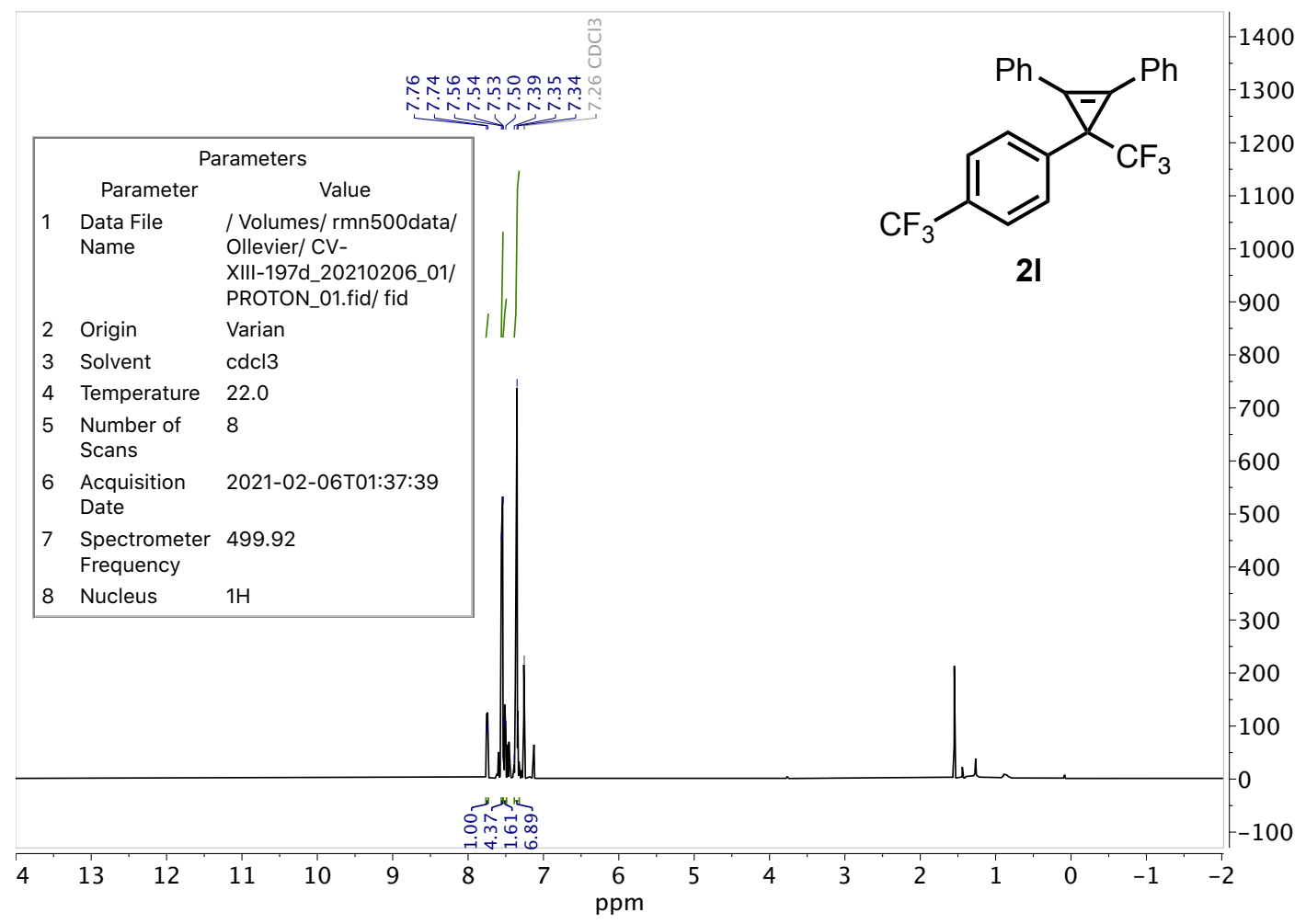




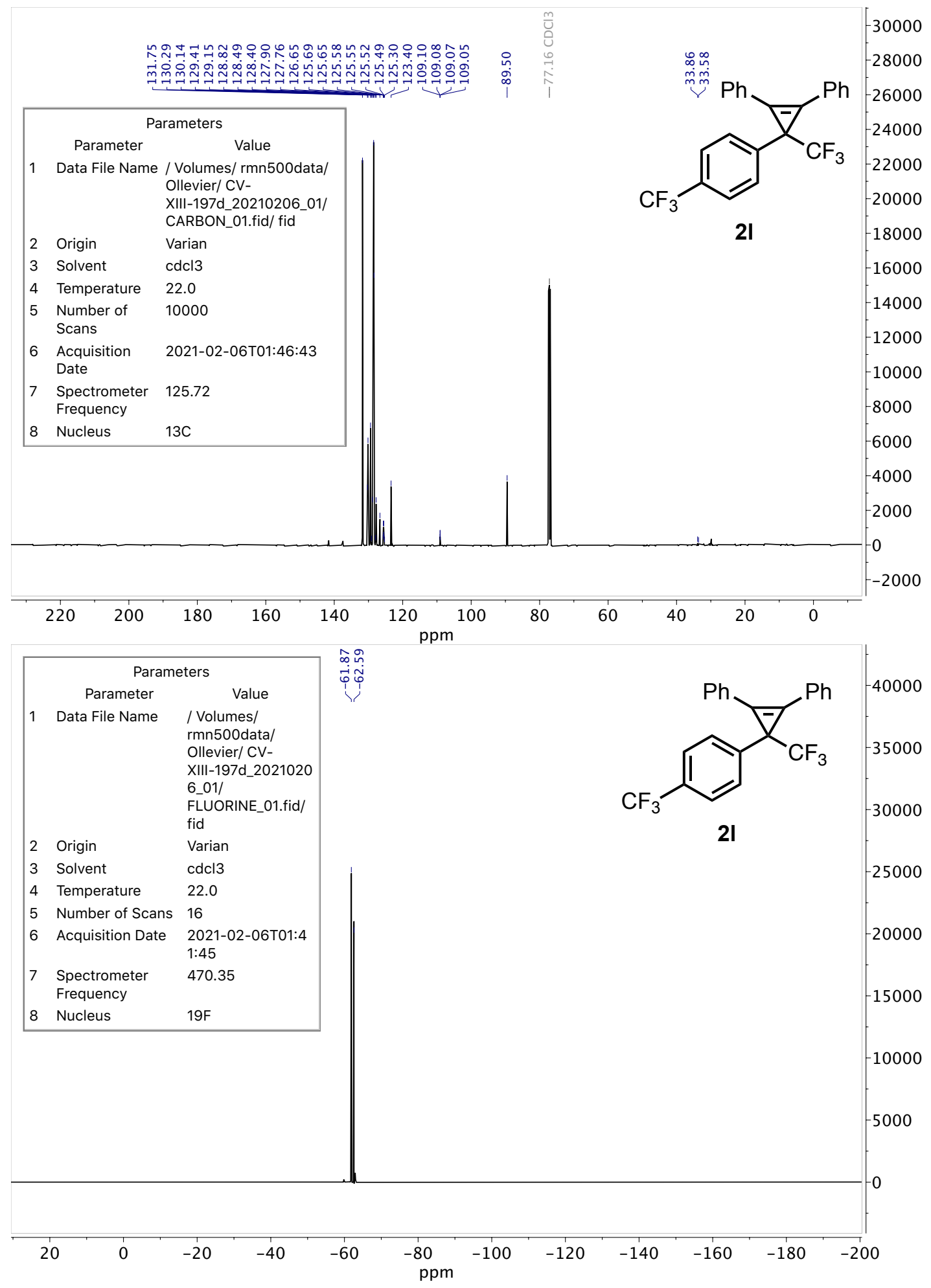




\section{4-(2,2,2-trifluoro-1-(pent-4-yn-1-yloxy)ethyl)-1,1'-biphenyl 3a}

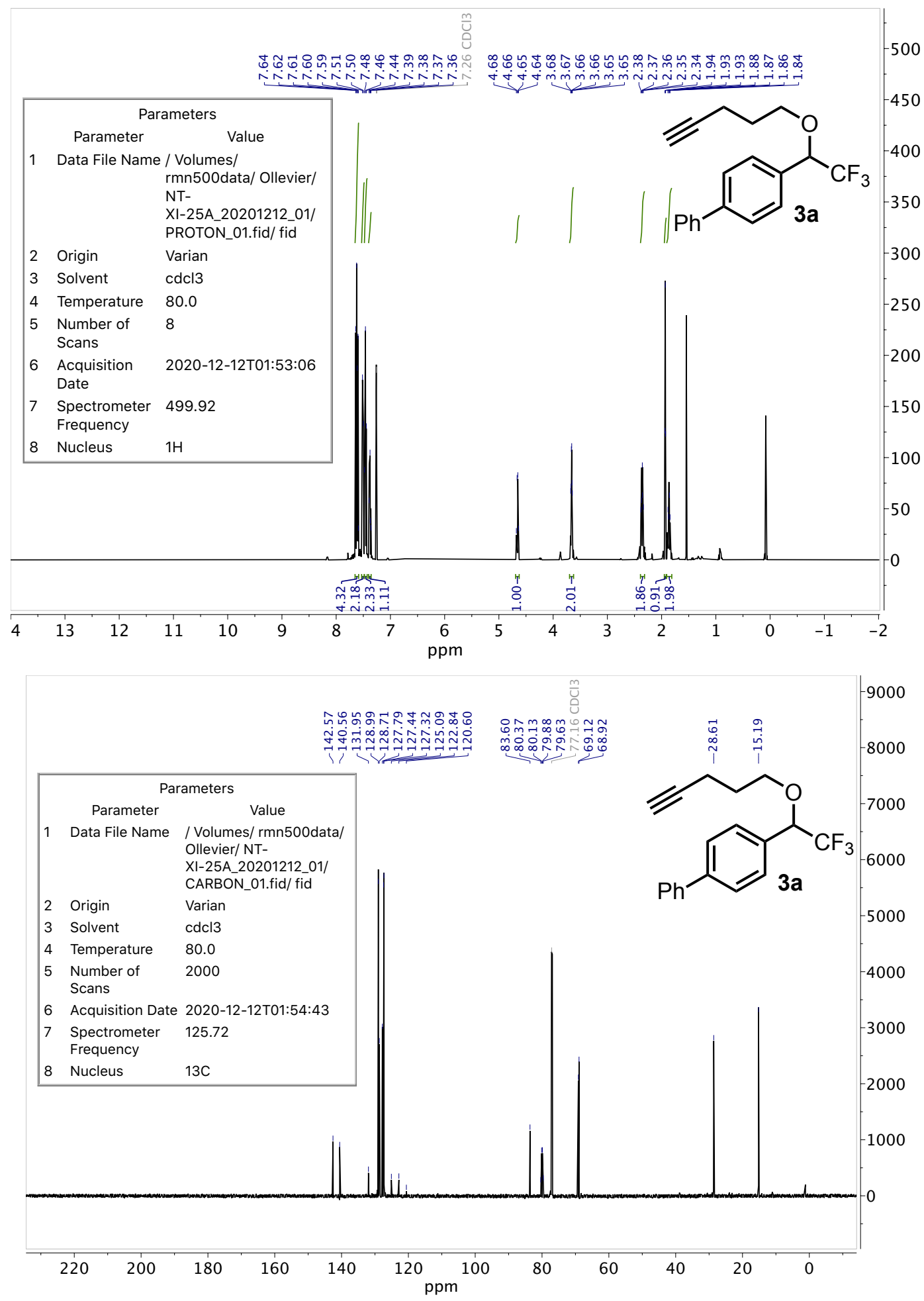




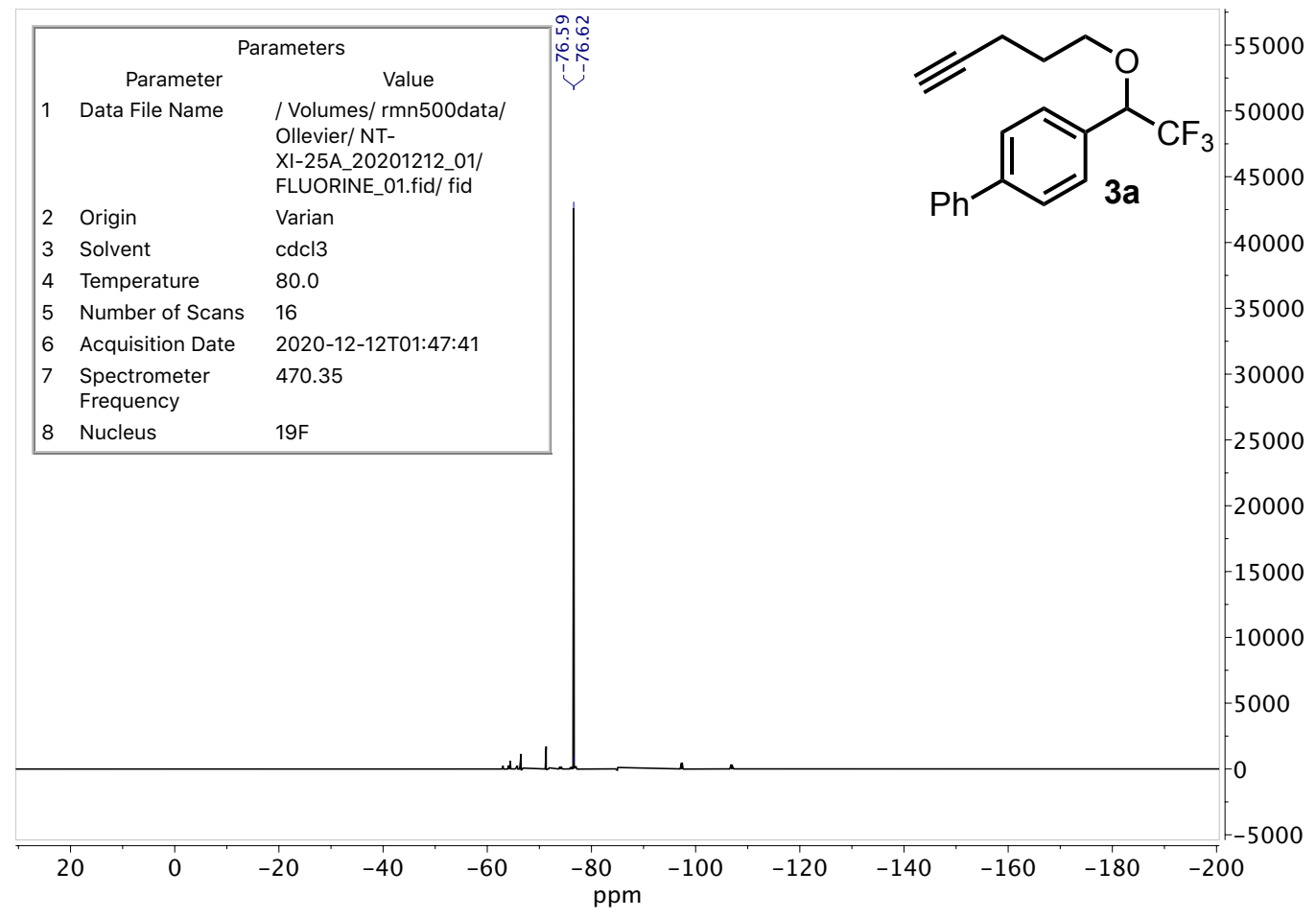

\section{4-(2,2,2-trifluoro-1-(prop-2-yn-1-yloxy)ethyl)-1,1'-biphenyl 3b}

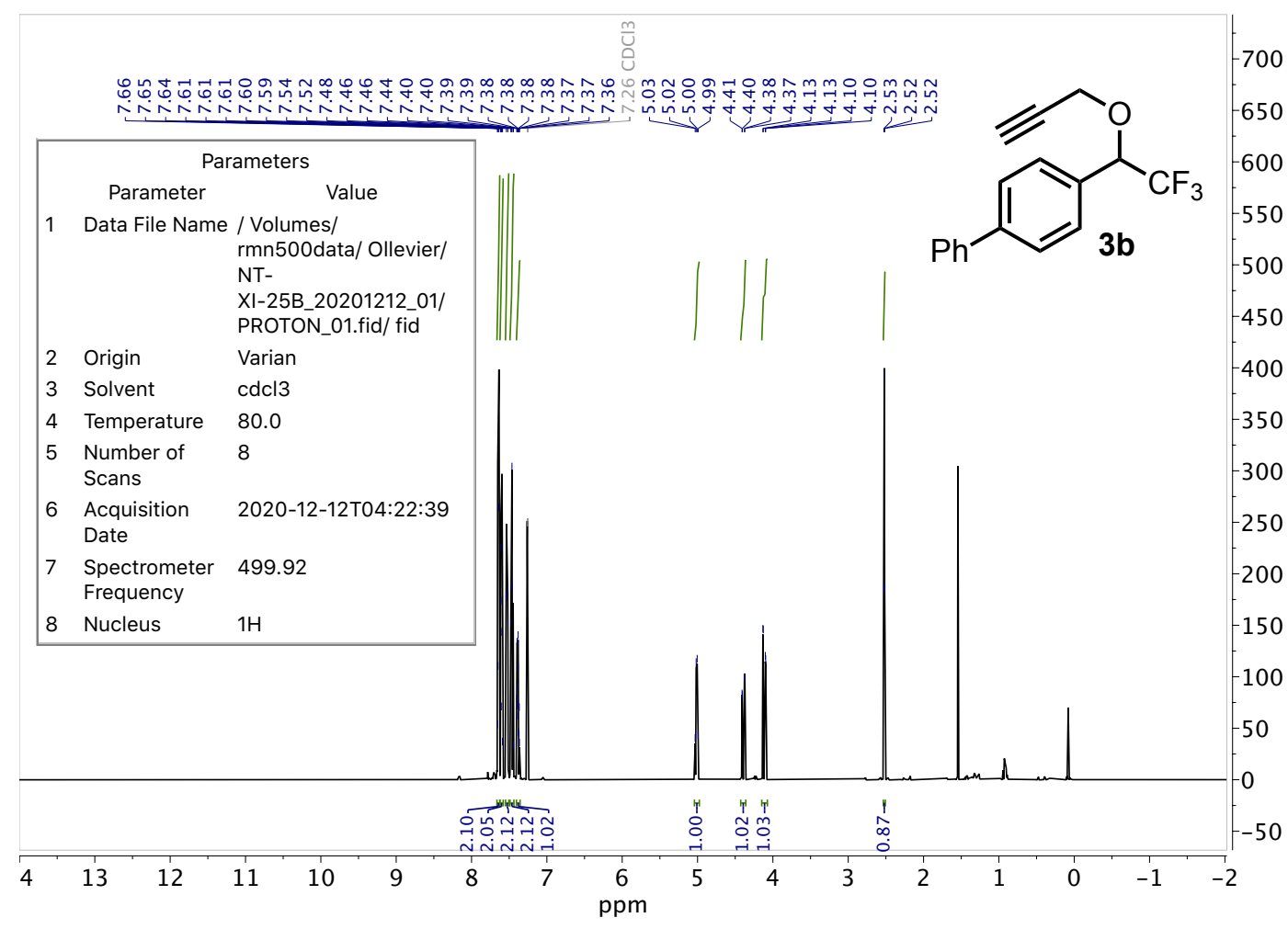




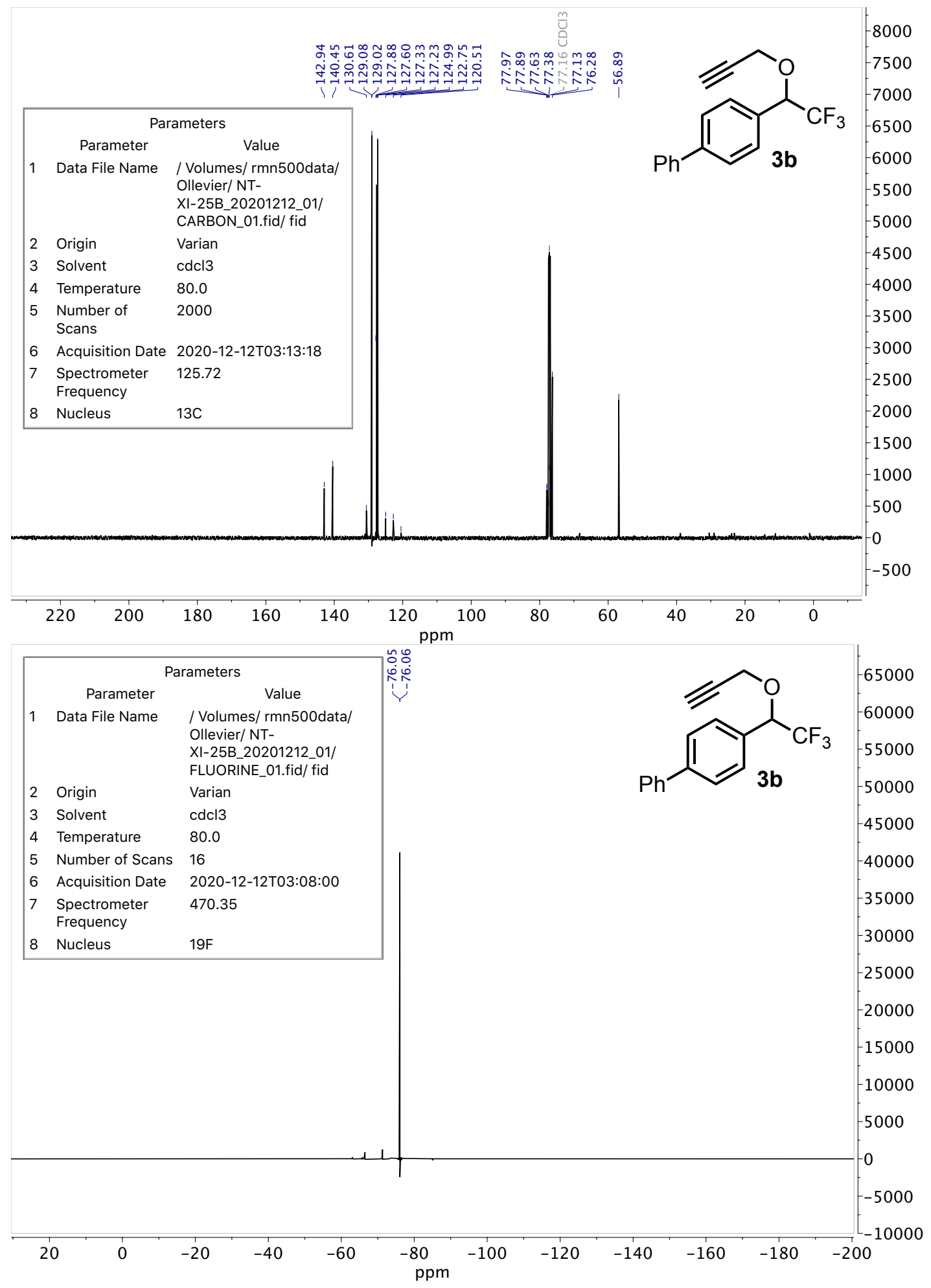




\section{4-(1-(Trifluoromethyl)cycloprop-2-en-1-yl)-1,1'-biphenyl 4ak}

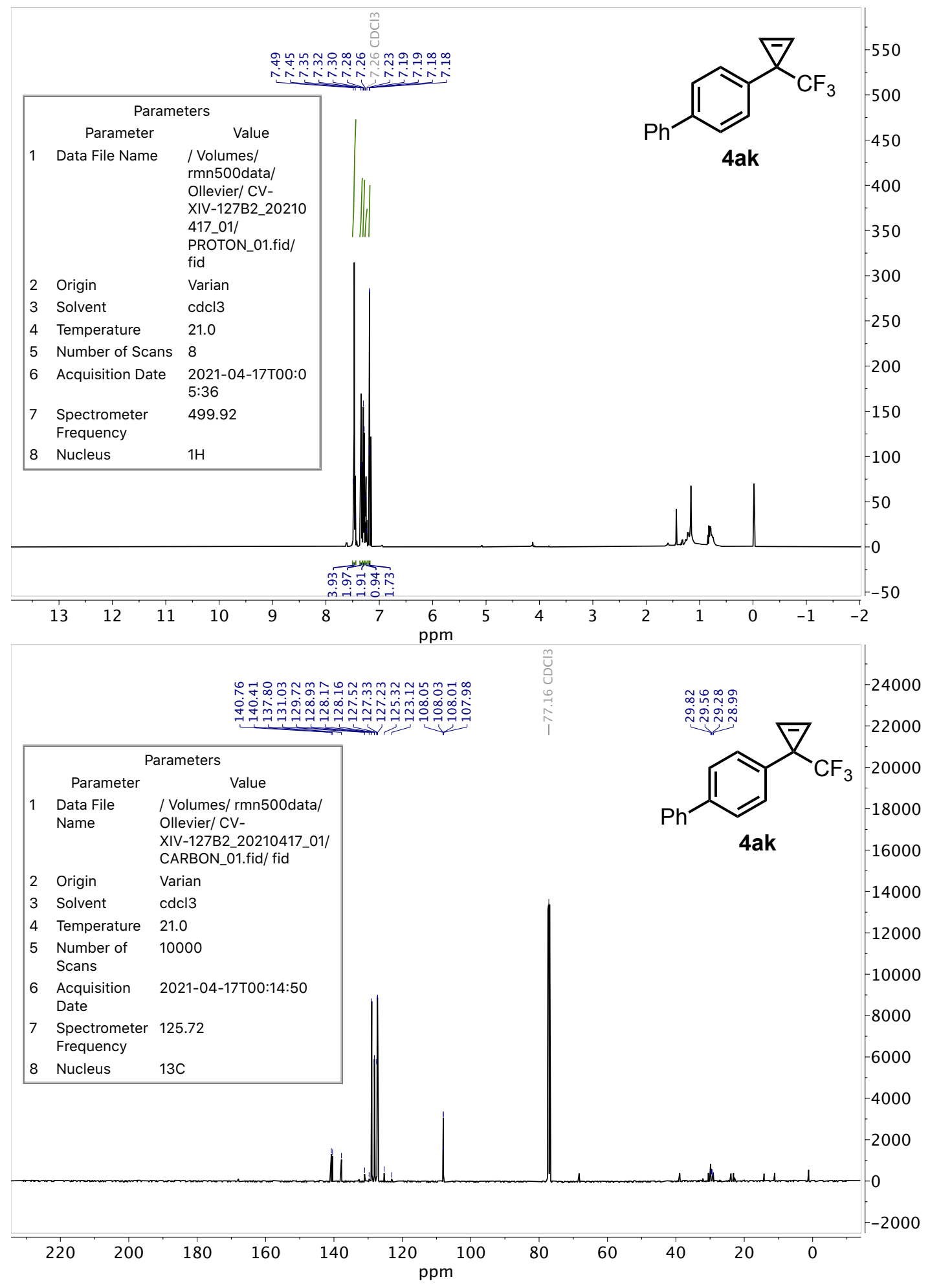

